\title{
Acoustic Room Impulse Response Shaping
}

by

Lakshmi Krishnan

\author{
A thesis \\ submitted to the Victoria University of Wellington \\ in fulfilment of the \\ requirements for the degree of \\ Doctor of Philosophy \\ in Engineering.
}

Victoria University of Wellington

2017 



\begin{abstract}
Impulse response shaping is a technique for modifying the characteristics of a linear channel to achieve desirable characteristics. The technique is well-known in the field of wireless communication. Acoustic impulse response shaping is used to reduce the effects of reverberation on audio signals propagating inside a room and is thus used for listening room compensation. This thesis addresses innovative approaches for acoustic impulse response shaping.

Many techniques have been proposed in the literature for canceling or reducing the effect of reverberation on the audio signal. Impulse response inversion attempts to completely cancel the effect of reverberations whereas impulse response shortening (or shaping) only partly equalizes the room impulse responses. Shortening has less stringent constraints than inversion and this can result in more robust solutions and thus more practically realizable systems.
\end{abstract}

Acoustic impulse response shaping works on measured room impulse responses and designs pre-filters to be placed before the loudspeakers so that the reverberation is reduced at the listening positions. When sampled, the room responses typically contain thousands or tens of thousands $(N)$ of samples. Thus, the shaping algorithm needs to be computationally fast and memory efficient in order to implement the system in real time. The techniques presented in the literature use interior point methods or steepest descent algorithms, which are computationally slow or require memory of the order of $N^{2}$. This thesis presents shaping approaches based on the Dual Augmented Lagrangian Method (DALM), known in the literature on sparse reconstruction for its super-linear convergence. The method presented here also makes use of the concept of a Forward Adjoint Oracle (FAO) to make the shaping algorithm memory efficient. Thus, the thesis presents computationally fast and memory efficient shaping algorithms that can be used for practically realizable systems.

The thesis also presents robust shaping approaches. The measured room responses may contain measurement errors or noise and can vary from time to time. 
These variations may be due to changes in atmospheric conditions (such as temperature or humidity) or due to change in position of objects inside a room. While design approaches over multiple microphone positions have been proposed for design of filters that are robust to change in microphone positions, a more rigorous approach is statistical, involving the inclusion of some statistical constraints into the optimization problem. The thesis presents both the approaches viz., a computationally faster version (using DALM) of the already proposed design over multiple positions and a statistically robust shaping formulation. The latter limits the probability of large errors between expected and obtained response to be less than a specified value. This ensures that the solution is robust to variations in the room response.

The shaping algorithm works in the time domain, shaping the temporal characteristics of the room response to a desired form. The frequency response of the shaped response can contain potentially undesirable peaks and troughs. This thesis therefore presents an approach for an efficient projection to improve spectral flatness of the resultant response. This algorithm can be combined with the fast and memory efficient DALM based approach to achieve joint time and frequency shaping.

Finally, the thesis also presents a computationally fast algorithm based on DALM for pressure matching used in sound field reproduction. Impulse response shaping is applied in sound field reproduction, showing that the levels of prereverberation induced by a temperature change can be reduced. This application is different from impulse response shaping approaches presented in the previous chapters and highlights the flexibility of the algorithm developed in this thesis and its wide range of applications. 


\section{Acknowledgments}

I take this opportunity to express my heartfelt gratitude and indebtedness to my primary supervisor Paul for his expert guidance, valuable suggestions, constant support, encouragement and above all, the understanding and wholehearted cooperation throughout the entire course of my thesis work, including the many difficult days. I also extend my cordial thanks and heartfelt gratitude to my secondary supervisor Terence for his guidance, valuable suggestions and also for the help extended to me at various phases of my thesis work.

I would like to thank Dr. Mark Poletti for providing me with valuable suggestions, especially an insight into acoustics which was crucial for a beginner like me to work on an acoustics problem. I extend my cordial thanks to Dr. Sudhir Singh for useful discussions on optimization formulations, which were very helpful for this thesis work.

I also wish to thank the staff at the School of Engineering and Computer Science for being caring and understanding while pulling out all the stops in providing me with support to carry out research during my early days as a mother. I extend my cordial thanks to past summer student at the School Mr. Reuben Jelleyman for his help in setting up the acoustic experimental models at the lab.

I take this opportunity to thank my partner Nandakumar and our little daughter Shreya for providing a great moral support and means of inspiration during the testing days of my thesis work. My words would be complete only by expressing my gratitude to my loving parents for their constant encouragement and moral support which have been critical in helping me stay on course.

Last not the least, I remember with prayers, the blessings showered on me by God Almighty without which I wouldn't have made it this far in my thesis work. 


\section{Contents}

1 Introduction 1

1.1 Properties of the room response . . . . . . . . . . . . . . . 4

1.2 Motivation . . . . . . . . . . . . . . . 5

1.3 Chapterwise summary . . . . . . . . . . . . . . . 8

1.4 Publications . . . . . . . . . . . . . . . . . . 10

2 Literature review I

(Acoustics) 13

2.1 Helmholtz equation . . . . . . . . . . . . . . . . . . 13

2.2 Characterization of diffuse field . . . . . . . . . . . . . . . 15

2.3 Image Source Method . . . . . . . . . . . . . . . . . . . . 15

2.4 Early reflections . . . . . . . . . . . . . . . . . . . . . . . . . . . . . . . . 17

2.5 Room response variations . . . . . . . . . . . . . . . . . . . . 17

2.6 Sound field reproduction . . . . . . . . . . . . . . . . . 19

2.7 Creation of sound zones . . . . . . . . . . . . . . . . 21

2.8 Evaluation of Direct to Reverberation Ratio . . . . . . . . . . . . . 22

2.9 Modelling of Head Related Transfer Functions . . . . . . . . . . . . 23

2.10 Chapter Summary . . . . . . . . . . . . . . . 24

\section{Literature review II}

(Shortening formulations and Optimization Algorithms) 25

3.1 Impulse Response Inversion . . . . . . . . . . . . . . . . . . 25

3.2 Impulse Response Shaping (or Shortening) . . . . . . . . . . . . . . 26

3.3 Perceptually enhanced shaping solutions . . . . . . . . . . . . 31

3.4 Robust implementation . . . . . . . . . . . . . . . . . . 33

3.5 Efficient sub-band implementation . . . . . . . . . . . . . 34

3.6 Optimization algorithms . . . . . . . . . . . . . . . 35

3.6.1 Steepest Descent Algorithm . . . . . . . . . . . . 35 
3.6.2 Interior Point Methods . . . . . . . . . . . . . . . . 36

3.6.3 Conjugate Gradient Descent Algorithm . . . . . . . . . . . . 36

3.6.4 Sparse Reconstruction Algorithms . . . . . . . . . . . . 37

3.7 Chapter Summary . . . . . . . . . . . . . . . . . . 38

4 Problem Formulation $\quad 41$

4.1 Shaping problem . . . . . . . . . . . . . . . 41

4.1.1 Definition of the weight vector . . . . . . . . . . . 43

4.2 Regularized shaping formulation . . . . . . . . . . . . . . . . . 46

4.3 Dual Augmented Lagrangian Method . . . . . . . . . . . . . . . . . . 49

4.4 Performance Metrics . . . . . . . . . . . . . . . . . . . 50

4.5 Chapter Summary . . . . . . . . . . . . . . . . . . . 52

5 Fast Algorithms for Shaping $\quad 55$

5.1 Background ....................... . . . 55

5.2 Dual Augmented Lagrangian Method . . . . . . . . . . . . . 56

5.2 .1 Special Cases . . . . . . . . . . . . . . 57

5.2 .2 Algorithm for D-2-1 objective function . . . . . . . . . . 58

5.2.3 Fast Dual Augmented Lagrangian Method . . . . . . . . . . 59

5.3 Fast algorithm for D- $\infty-1$ primal objective . . . . . . . . . . . . 60

5.4 Fast algorithms for $\ell_{2}$ regularised objective functions . . . . . . . . 62

5.4 .1 D-2-2 algorithm . . . . . . . . . . . . . . . . . 62

$5.4 .2 \quad \mathrm{D}-\infty-2$ algorithm . . . . . . . . . . . . . . . 63

5.5 Forward Adjoint Oracle Approach . . . . . . . . . . . . . . . . . . 64

5.5.1 Implementation of $C h$ using Fast Fourier Transforms . . . . 64

5.5.2 Implementation of $C^{\mathrm{T}} y$ using Fast Fourier Transforms . . . . 65

5.6 Design over multiple microphone positions . . . . . . . . . . . . 66

5.7 Simulation Results . . . . . . . . . . . . . . . . . . 67

5.8 Experiments in the lab . . . . . . . . . . . . . . . . . 74

5.8.1 Robustness to atmospheric conditions . . . . . . . . . 79

5.9 Contributions from this chapter . . . . . . . . . . . . . . 81

6 Joint Time and Frequency Shaping 83

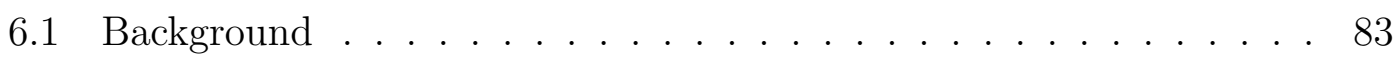

6.2 Problem Formulation . . . . . . . . . . . . . . . . . . . 84

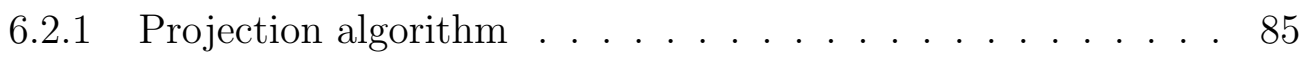

$6.2 .2 \quad$ Special Cases . . . . . . . . . . . . . . . . . 88

6.2.3 Time and frequency shaping algorithm . . . . . . . . 88 
6.3 Robust time and frequency shaping algorithm . . . . . . . . . . 89

6.4 Simulation results . . . . . . . . . . . . . . . . . . . . . . . . 90

6.5 Contributions from this chapter . . . . . . . . . . . . . 99

7 Statistically Robust Shaping Formulation 101

7.1 Background . . . . . . . . . . . . . . . . . 101

7.2 Problem Definition . . . . . . . . . . . . . . . . . . 102

7.2.1 Conversion to a convex problem . . . . . . . . . . . 103

7.3 Simulation Results . . . . . . . . . . . . . . . . . . . 107

7.4 Contributions from this chapter . . . . . . . . . . . . 111

8 Pressure Matching Robust to Temperature Change 113

8.1 Background . . . . . . . . . . . . . . . . . 113

8.2 Temperature robustness . . . . . . . . . . . . . . . 115

8.3 Sound Field Reproduction . . . . . . . . . . . . . . . . . . . . 118

8.3.1 Inverse filter solution . . . . . . . . . . . . . . . . . . 121

8.3.2 Impulse response shaping solution . . . . . . . . . . . . . . . 121

8.4 Relevant Solutions . . . . . . . . . . . . . . . . . . . 123

8.4.1 Steepest Descent Method . . . . . . . . . . . . . . . 123

8.4.2 Conjugate Gradient Descent Algorithm . . . . . . . . . . . . 123

8.4.3 Variant of Fast D-2-2 algorithm . . . . . . . . . . . . . . . 124

8.5 Forward Adjoint Oracle Approach . . . . . . . . . . . . . . . . . . . 125

8.5.1 Implementation of $\mathcal{C} h$ using Fast Fourier Transforms . . . . 125

8.5.2 Implementation of $\mathcal{C}^{\mathrm{T}} y$ using Fast Fourier Transforms . . . . 126

8.6 Simulation results . . . . . . . . . . . . . . . . . 126

8.7 Contributions from this chapter . . . . . . . . . . . . . 133

9 Conclusion $\quad 135$

9.1 Original contributions . . . . . . . . . . . . . . 136

9.2 Future Work . . . . . . . . . . . . . . . . . . 139

A Preliminaries for Deriving Dual Augmented Lagrangian Method141

A.1 Fenchel Duality Theorem . . . . . . . . . . . . . . . . . . . . . 141

A.1.1 Dual problem of an $\ell_{1}$ regularised objective function . . . . . 142

A.1.2 The Fenchel Conjugate of $\|x-b\|_{2}^{2} / 2 \lambda \ldots \ldots$. . . . . . . . 143

A.1.3 The Fenchel Conjugate of $\|x-b\|_{\infty} \ldots \ldots . . . . . . . .143$

A.2 Soft Thresholding function . . . . . . . . . . . . . . . . . . 144 
B Preliminaries for the Statistical Model

B.1 Error variance for Independent Identically Distributed Gaussian perturbations . . . . . . . . . . . . . . . . . 145

B.2 Statistically robust model as a Second Order Cone Program . . . . 148

List of Abbreviations 


\section{Chapter 1}

\section{Introduction}

Sound is a manifestation of mechanical energy [1, ch. 1]. Travelling as pressure waves, sound waves propagate through a medium as vibrations, transmitting the mechanical motion from one particle to another. As a sound wave travels, it is likely to undergo reflection from barriers resulting in phenomena such as echoes. A reflection of a shout from a wall in a large hall (say, $17 \mathrm{~m}$ in length) will reach the person who made the shout, in about $0.1 \mathrm{~s}$ time and such an echo will be distinctly heard $[2,3]$. In contrast to a distinct echo, the term reverberation is often applied to reflected sound that reaches the listener before $0.1 \mathrm{~s}$ of round trip delay. Reverberation, in general, is defined as the persistence of sound after a sound disturbance is produced [4]. Reverberation could build up because of the reflections from multiple reflectors, and decay as the sound is absorbed by the surfaces of the different objects in the space such as furniture, people and air. Reverberation persists even after the sound source is switched off. In detection systems like radar and sonar, special techniques are deployed to overcome the effects of reverberation [5] which otherwise mitigates the detection and interpretation of the received echoes.

On the other hand, it is interesting to note that reverberation is a ubiquitous component of the tool kit of a an expert music composer. Natural reverberation often plays a vital role in classical musical concerts, where a carefully designed concert hall utilizes the persistence of reverberation in the hall to enhance the sound from the performing orchestra $[6,7]$. It is understood that the reverberation effects created by ancient monuments and heritage buildings or even within caves were used by sages and monks to make their rituals more alluring and effective [8]. What has made the reverberation so significant in keeping the listener spell 
bound? It is widely accepted that the human brain can perceive the geometry of the room (where the music is being played) from the reverberations. The audio cues then play a major role in shaping the emotional response of the listener. One can imagine by contrast how unappealing the same music is when played in an anechoic room. While humans prefer different combinations of melodies and rhythms, which are matters of personal choice which may change with region and over generations, reverberation seems to be appreciated by all. A performance by a non-expert singer can become professional with the magic hand of an expert sound engineer. The sound engineer uses a variety of techniques such as fading, compression, expansion, equalizing and filtering to simulate reverberation effects, to be added carefully, while mixing the sound to be rendered to the audience [7]. Thus, reverberation is inextricably linked to the day-to-day functioning of music recording studios and concert halls.

Reverberation is carefully assessed and allowed for in the design of rooms and auditoriums due to the well known effects of reverberation on the sonority of the sound received. Special care is taken in architectural designs to ensure that sound waves are properly deflected during its propagation and optimally absorbed into special roof and wall lining materials to enhance the listener experience [7].

In some specialist acoustic applications, however, reverberation does not necessarily lend itself to a positive listening experience. In listening devices such as speech amplifiers, reverberation components are unacceptable due to their potential negative effects on speech intelligibility. (Speech intelligibility is a measure of the comprehensibility of speech in given conditions [9-11].) Reverberation blurs the speech sounds over time; masking stops, glides and vowel transitions and prosodic cues such as pitch and duration [12]. However, certain portions of the reverberation, called the early reflections, are found to perceptually enhance the speech intelligibility $[13,14]$. Thus, reverberation is to be selectively suppressed in such applications.

Techniques are available to design equalizers in such a way that the reverberations are selectively suppressed at the receiver, thereby making the reception more enjoyable. The surround sound technology $[1,15]$ widely available now uses arrays of microphones to selectively combine the sound received. The front channels in surround systems are designed to pick up less reverberation, in contrast to the surround microphones. Such systems normally deploy a variety of effective patterns 
for the microphone deployment. However, these systems do not directly attempt to cancel the effects of room responses. Instead, they reduce the unwanted effects of reverberation. Surround sound technology is an active research area with research prospects in a wide range of topics ranging from the materials used for the walls of the room to signal processing techniques used for listening room compensation $[16,17]$.

Many techniques of listening room compensation have been proposed in the literature for canceling or reducing the effects of reverberations on the signal [17]. Such techniques aim to find filters that are placed before the loudspeakers in an end to end signal path from the source via the loudspeaker to the listener. These filters are designed to compensate for the room impulse response. Every room is characterized by a room impulse response that provides a simple representation of linear distortions (reverberations) [4] encountered by a signal propagating inside the room.

The pre-filters compensate for an estimate of the channel between the loudspeakers and listener so that the original signal from the loudspeaker is received by the listener with minimum distortion. Impulse response inversion aims to cancel the effect of the room response [18]. This technique is very sensitive because the room response is non-stationary and can vary with positions inside the room as well as with changes in atmospheric conditions (such as temperature and humidity). It is difficult to design an inverse filter that is robust to these conditions. Impulse response shaping or shortening [19-22] finds application in such a scenario. The technique aims to partly equalize the impulse response so as to achieve a desired form. Impulse response shaping is well known in the field of wireless communication as being applied to discrete multi-tone receivers to control bit error rate [23-26]. Using shaping, the impulse response can be made to fit within the duration of the cyclic prefix of a symbol. In acoustics, room impulse response shaping aims to reduce the perceptible effects of reverberation on the signal, specifically by reducing the late reverberations more whilst allowing some early reflections which are perceptually useful [27]. Shaping is thus an approximation to inversion and has less stringent constraints, which is helpful in the creation of more robust and practically realizable systems.

When there are multiple signal paths as in the case of multiple loudspeakers and multiple listening position systems, the pre-filters perform shaping to reduce 
reverberation as well as cancel the cross-talk between loudspeaker signals. Thus, pre-filters that solve the joint problem of room response shaping and cross-talk cancelation are estimated and implemented in multichannel room impulse response shaping $[21,27,28]$. In this case, $L M$ pre-filters are to be estimated where $L$ is the number of loudspeakers and $M$ is the number of microphones in the cross-talk canceler setup.

We also propose another application of multichannel shaping to pressure matching, in which the sound pressure values at specific points inside a room are matched to a desired response. This is a different arrangement and requires only $L$ prefilters. This approach is based on the assumption that a virtual source produces similar responses at certain synthesized positions. These positions are typically chosen to be a sphere around the original microphone position. The pressure matching technique uses shaping formulations that include characterization of sound fields at those positions. Such a formulation is consistent with the wave equation.

Acoustic impulse response shaping formulations rely on the room impulse responses, which include the linear effects of the room on a signal propagating inside

it [4]. In order to develop a meaningful optimization formulation for shaping, it is essential that the properties of the room impulse responses are well understood. The following section discusses in brief some of the properties of the room response that are essential for appreciating the scope and relevance of the shaping formulations.

\subsection{Properties of the room response}

The room impulse response is a representation of the linear effects of the room on a signal propagating through it, such as floor and wall reflections. The temporal structure of the room response includes an approximately exponential decay, with most of the energy concentrated in the first few milliseconds. The room response can be broadly classified into three parts: a direct path component, early reflections and late reverberation.

The signal arriving directly without undergoing any reflection is the direct path component. The direct path is followed by the early reflections, the delay of which depends on the size of the room. But early reflections are often considered to be 
within 50 milliseconds [4]. The early reflections have been found to be very useful in maintaining the perceptual quality of the audio signal and hence significant research has been concentrated into studying their characteristics and importance in perceptual quality $[13,14]$. This is the main motivation for the performance metric called $D_{50}$, which is the ratio of energy in first 50 milliseconds to the total energy of the room response, in analyzing the efficacy of impulse response shortening approaches $[21,28,29]$. The portion of the room response arriving late are called late reverberations, which, though they are of less energy, can seriously degrade the perceptual aspects of the signal. Late reverberation is the key component of the room response which is to be reduced by room impulse response shortening techniques.

Another important property of room reverberations is the reverberation time, denoted as $T_{60}$ [30], which is the time in milliseconds required for the room response to reduce to $60 \mathrm{~dB}$ less than the direct component. The weighting function used in impulse response shaping formulations is designed based on $D_{50}$ and $T_{60}$, so that the estimated shaping filters reduce the early reflections only lightly if at all, while significantly reducing the late reflections beyond a modified $T_{60}$.

\subsection{Motivation}

Acoustic room impulse response shaping is a research problem that involves the study of both room acoustics and signal processing. Room acoustics is an evolving and active field of study that has scope for a wide range of applications, including modeling of the room acoustic properties and signal processing applications to manipulate these properties. Promising research areas are 3D-audio and multi-zone surround sound systems; acoustic room impulse response shaping is applicable to both of these.

The existing techniques in impulse response shaping involve the use of an optimization formulation, that involves minimization of some norm of the weighted error between the desired response and the response obtained using shortening filters [27]. The choice of the norm is critical, as it affects both the nature of the errors and the computational efficiency. The previous works in shaping use 
either an $\ell_{\infty}$ norm ${ }^{1}[27,31]$ or an $\ell_{p}$ norm ${ }^{2}[21,28,29,32]$ and use either interior point methods [27] that are memory exhaustive or gradient descent approaches $[21,28,29,32]$ that are computationally very slow.

This thesis uses a regularized [33] formulation of the shaping problem which minimizes a combination of a norm of the weighted error and a regularizer of the shaping filters. Both $\ell_{1}$ and $\ell_{2}$ norm regularizers are proposed to be used in this work from the observation that the $\ell_{1}$ norm results in sparse filters while the $\ell_{2}$ norm reduces the energy of the filters. Regularization ensures that the filter taps are not high in magnitude, providing an inherent robustness to the designed filters.

An important design criterion of a practically realizable shaping system is the computational speed of the algorithm used to estimate the shaping filters. This is because the room responses are typically long (of the order of 1000s or $10000 \mathrm{~s}$ of samples) resulting in huge optimization problems. This thesis proposes a computationally fast approach to solve the joint problem of shaping and cross-talk cancelation. The algorithm used in this work for solving the minimization problem is the Dual Augmented Lagrangian Method (DALM) [34-36], known in the field of sparse reconstruction [37] for its super-linear convergence [35]. The thesis presents different variants of DALM formulations depending on the choice of the norm of the weighted error and the regularizer. Thus, a formulation suited for a situation can be chosen, for example, an $\ell_{2}$ regularizer if low energy filters are desired and implemented in a real scenario.

Another important consideration for a good shaping algorithm is robustness. This is because the room responses used in the filter estimation are measured and can contain measurement errors or noise. In addition, they vary with position inside the room due to constructive and destructive interference of propagating sound waves on the direct component at the sound location of interest. The variations can also result from movement of objects in the room, or opening or closing of a door of the room, or with atmospheric conditions such as the temperature and humidity inside the room. The filters that are to be estimated to reduce or cancel the effect of room reverberations are thus distinctive to specific locations and specific conditions. It is challenging to design filters that are robust to all such changes. The filters designed according to recent works [21, 27-29, 31, 32] are

\footnotetext{
${ }^{1}\|x\|_{\infty}=\max _{n} x(n)$, where $n=1,2, \ldots N, N$ is the length of vector $x$

${ }^{2}\|x\|_{p}=\sqrt[p]{x(1)^{p}+x(2)^{p}+\ldots x(N)^{p}}, N$ is the length of vector $x$
} 
not robust to changes in room responses. An important theoretical framework for analyzing the effect of errors of inaccurate filtering due to changes in positions has been established by Radlovic et.al. [38] which highlights the need for a robust filter that can be applied at various positions with less error. Some work in this direction is discussed in [39], where the authors present a shaping filter design approach over multiple microphone positions. This involves generation of multiple perturbed channels corresponding to changes in position and estimating a filter that performs well at all these positions. But it is computationally challenging, resulting in a difficult optimization problem. This thesis also implements this approach, but in a computationally faster manner using the DALM algorithm.

A more rigorous approach is to design filters which are robust to many of these variations according to a statistical criterion. The statistical formulation includes probabilistic constraints into the optimization problem, for example, limiting the probability of error above a threshold to an assured low probability [40]. This requires the development of an error model of the result of filtering using perturbed microphone signals. A statistically robust formulation for the shaping problem is discussed in the thesis, in which the original non-convex probabilistic optimization formulation has been reformulated as a standard Second Order Cone Program (SOCP) [41].

Another important criterion for good shaping algorithm is frequency flatness. A good equalization algorithm will seek to flatten the frequency response. The shaping technique is generally applied in the time domain and can allow peaks and troughs to appear in the frequency response curve of the shaped responses. Frequency domain control can be included in the formulation to ensure a uniform behaviour in the frequency domain. Some work in this direction is discussed in [28], but the solution algorithm is based on gradient descent and is computationally slow. The thesis discusses an approach for efficient projection onto a low-dimensional norm ball which can be used to impose the frequency constraints. This approach can be easily combined with the DALM formulation and can be used as a computationally fast algorithm for joint time and frequency shaping.

As seen in the previous section, a pressure matching formulation is another application of the algorithm mentioned above. While exploring pressure matching formulations in literature, it is interesting to note that no shaping formulation has been reported in the literature for the sound field reproduction problem. Ad- 
dressing this gap, this thesis presents a computationally fast pressure matching algorithm using a modification of the $\ell_{2}$ regularized $\ell_{2}$ DALM formulation. The computational performance of this algorithm is established by comparing it with the conventional Tikhnov regularized formulation which is solved using a steepest descent algorithm. The application of the DALM formulation developed in the thesis to this totally different formulation highlights the flexibility of the algorithm and suggests that it could be used in a wide range of applications.

From the discussions so far, it follows that though there is an extensive body of research on the control of room acoustics, there are some important gaps in the developments so far. These were the main motivating factors in taking up this dissertation. The thesis specifically focusses on the development of the algorithms for impulse response shaping with real time implications. The main findings of the present work are reported in nine chapters, summarized in the section to follow.

\subsection{Chapterwise summary}

CHAPTER 2: This chapter discusses the key literature in the field of acoustics that is essential to appreciate the work presented in this thesis.

CHAPTER 3: Key literature relating to the optimization formulation of the shaping problem are discussed in this chapter. The chapter also discusses literature relating to some optimization algorithms used to solve the formulations.

CHAPTER 4: The shaping formulation used in this thesis is explained in detail in this chapter. Details such as the formulation as a regularized minimization problem, the various norms used and the weighting function are detailed. This facilitates understanding of the derivations and the results presented in the following chapters.

CHAPTER 5: This chapter explains the algorithms developed in this thesis for estimating the shaping filters in a computationally fast manner. The algorithms used are variants of the Dual Augmented Lagrangian Method, known in sparse reconstruction. One of the key contributions from this thesis is the development of a fast DALM algorithm for solving the $\ell_{1}$ regularized $\ell_{\infty}$ norm minimization problems, which is advantageous for the development of a shaping implementation 
in a real scenario. The efficacy of the DALM algorithm in shaping is verified using simulation and real time experimental studies. The chapter also presents fast DALM algorithms for some other regularized formulations that can be useful for the problem, specifically, $\ell_{1}$ regularized $\ell_{2}$ norm minimization, $\ell_{2}$ regularized $\ell_{2}$ norm minimization and $\ell_{2}$ regularized $\ell_{\infty}$ norm minimization. The performance of these algorithms are also experimentally validated. In addition, the chapter also presents a modification of the formulations to a design over multiple positions to achieve empirical robustness to microphone position errors. The empirical design approach was introduced by Jungmann et al. in [39] but the algorithm for finding the shaping filters based on steepest descent is computationally slow. A faster version of this approach based on fast DALM is presented in this chapter along with experimental validation.

CHAPTER 6: This chapter presents an approach for joint time and frequency shaping. The algorithms presented in the previous chapters design shaping filters based in the time domain and can therefore result in undesired peaks and troughs in the frequency spectra of the filtered responses. An effective shaping algorithm should achieve both time domain shaping and frequency domain flattening of the resultant responses. An efficient approach for projection onto a low dimensional norm ball is presented in this chapter, which can be used to achieve the frequency flatness. The DALM algorithm is a proximal minimization algorithm [35] and this projection step can be easily combined with DALM as an additional projection. Such an algorithm is used in this thesis to achieve joint time and frequency shaping. This is explained in this chapter along with experimental validation. The extension of the algorithm to design over multiple microphone positions to achieve spatial robustness is also presented.

CHAPTER 7: A statistically robust formulation for the shaping problem is presented in this chapter. The method uses a stochastic model of the channel variations to explicitly limit the probability of large deviations from the desired performance. This results in the formulation of a complicated optimization formulation which also requires knowledge of the error model. The derivation of the optimization formulation and its conversion to a standard convex problem using some approximations are described in this chapter. The performance of the method is evaluated on realistic channel perturbations and the resulting shaped responses are shown to comply with the robustness specification. 
CHAPTER 8: The pressure matching formulation of an Active Compensated Sound Field Reproduction (AC-SFR), which is an entirely different concept, is discussed in this chapter. The DALM formulations developed for shaping can be used to obtain computationally fast solutions to solve the pressure matching problem, which are discussed in this chapter. This highlights the flexibility and wide applicability of the algorithms developed in this thesis.

CHAPTER 9: This chapter summarizes the conclusions from this thesis and discusses the scope for future work.

\subsection{Publications}

The publications from this thesis are listed below: 
Table 1.1: Publications for this thesis.

\begin{tabular}{|c|c|c|c|c|c|}
\hline Title & Authors & $\begin{array}{l}\text { Conference/ } \\
\text { Journal }\end{array}$ & $\begin{array}{l}\text { Page } \\
\text { count }\end{array}$ & $\begin{array}{l}\text { Chapter } \\
\text { number }\end{array}$ & Status \\
\hline $\begin{array}{l}\text { A sparsity based } \\
\text { approach to Acoustic } \\
\text { Impulse Response } \\
\text { Shaping }\end{array}$ & $\begin{array}{l}\text { Krishnan, L., } \\
\text { Teal, P., } \\
\text { Betlehem, T. }\end{array}$ & $\begin{array}{l}\text { IEEE SSP } \\
2014\end{array}$ & 4 & 5 & Published \\
\hline $\begin{array}{l}\text { A robust sparse } \\
\text { approach to Acoustic } \\
\text { Impulse Response } \\
\text { Shaping }\end{array}$ & $\begin{array}{l}\text { Krishnan, L., } \\
\text { Teal, P., } \\
\text { Betlehem, T. }\end{array}$ & $\begin{array}{l}\text { IEEE ICASSP } \\
2015\end{array}$ & 5 & 5 & Published \\
\hline $\begin{array}{l}\text { Fast algorithms } \\
\text { for Acoustic Impulse } \\
\text { Response Shaping }\end{array}$ & $\begin{array}{l}\text { Krishnan, L., } \\
\text { Teal, P., } \\
\text { Betlehem, T. }\end{array}$ & $\begin{array}{l}\text { IEEE Trans. } \\
\text { Audio, Speech } \\
\text { \& Language } \\
\text { Processing }\end{array}$ & 11 & 5 & $\begin{array}{l}\text { Under } \\
\text { revision }\end{array}$ \\
\hline $\begin{array}{l}\text { Efficient projection } \\
\text { onto a low dimensional } \\
\ell_{2} \text { norm ball }\end{array}$ & $\begin{array}{l}\text { Teal, P., } \\
\text { Krishnan, L., } \\
\text { Betlehem, T. }\end{array}$ & $\begin{array}{l}\text { J. Engineering } \\
\text { Optimization }\end{array}$ & 10 & 6 & $\begin{array}{l}\text { Under } \\
\text { revision }\end{array}$ \\
\hline $\begin{array}{l}\text { A statistically robust } \\
\text { approach to Acoustic } \\
\text { Impulse Response } \\
\text { Shaping }\end{array}$ & $\begin{array}{l}\text { Krishnan, L., } \\
\text { Betlehem, T. } \\
\text { Teal, P., }\end{array}$ & $\begin{array}{l}\text { IEEE Signal } \\
\text { Processing } \\
\text { Letters }\end{array}$ & 5 & 7 & Published \\
\hline $\begin{array}{l}\text { Temperature robust } \\
\text { Active-compensated } \\
\text { Sound field Reproduction } \\
\text { using Impulse Response } \\
\text { Shaping }\end{array}$ & $\begin{array}{l}\text { Betlehem, T. } \\
\text { Krishnan, L., } \\
\text { Teal, P., }\end{array}$ & $\begin{array}{l}\text { IEEE Signal } \\
\text { Processing } \\
\text { Letters }\end{array}$ & 5 & 8 & $\begin{array}{l}\text { Under } \\
\text { review }\end{array}$ \\
\hline
\end{tabular}




\section{Chapter 2}

\section{Literature review I \\ (Acoustics)}

A comprehensive understanding of the principles of acoustics and important acoustic problems is essential for this research work. This chapter describes some relevant research topics in acoustics, their formulations and significance. Some important acoustic research problems considered in this chapter include sound reproduction, acoustic contrast control, characterization of sound fields and modeling of head related transfer functions (HRTF).

\subsection{Helmholtz equation}

The pivotal relation in acoustics, which is used in many formulations of applications, is the Helmholtz equation [4]. For a single frequency, this is given by

$$
\nabla^{2} p+k^{2} p=0
$$

where $p$ is the acoustic pressure, $k=\omega / v$ is the wave number and $v$ is the speed of sound in the medium considered. The Helmholtz equation describes the sound propagation and acoustic pressures at different points in the medium. In one dimension, the Helmholtz equation is $\frac{\partial^{2} p}{\partial x^{2}}+k^{2} p=0$, the general solution of which is given by

$$
p(x, t)=F(v t-x)+G(v t+x)
$$

where $F$ and $G$ are arbitrary functions for which second derivatives exist. $F(v t-x)$ represents a pressure wave travelling in the positive $x$-direction with a velocity $v$ and $G(v t-x)$ a pressure wave in the negative $x$-direction. The sound pressure $p$ is constant in any plane perpendicular to the $x$-axis. These planes of constant sound 
pressure are called wavefronts and any line perpendicular to them is a wave normal.

The particle velocity of a plane wave has only one non-vanishing component which is parallel to the $x$-axis given by

$$
v(x, t)=\frac{1}{\rho_{0} v}(F(v t-x)-G(v t+x))
$$

It can be seen from (2.2) and (2.3) that the ratio of sound pressure and particle velocity $p / v=\rho_{0} v$ in a plane wave propagating in the positive direction $(G=0)$ is frequency independent. This ratio is called the characteristic impedance of the medium. For example, for air at $20^{\circ} \mathrm{C}, \rho_{0} v=416 \mathrm{~kg} \mathrm{~m}^{-2} \mathrm{~s}^{-1}$.

A progressive plane harmonic wave is a plane wave in which the time and space dependence of the sound pressure follows a sinusoid function. If we set $G=0$ and $F$ to be complex sinusoid, we obtain

$$
p(x, t)=\hat{p} e^{j(\omega t-k x)}
$$

where $\hat{p}$ is the amplitude of the wave and $k$ is the wave number.

The Helmholtz equation for the three dimensional case in spherical co-ordinates is given by

$$
\frac{\partial^{2} p}{\partial r^{2}}+\frac{2}{r} \frac{\partial p}{\partial r}=\frac{1}{v^{2}} \frac{\partial^{2} p}{\partial t^{2}}
$$

whose solution is

$$
p(r, t)=\frac{\rho_{0}}{4 \pi r} Q(t-r / v)
$$

which represents a spherical wave produced by a point source at $r=0$ with volume velocity $Q$, which is the rate at which fluid is expelled by the source. $t-r / v$ indicates that the strength of any disturbance created by the sound source propagating outward with velocity $v$ decreases as $1 / r$. If the volume velocity of the source varies according to $Q(t)=\hat{Q} e^{j \omega t}$ with $k=\omega / v,(2.6)$ represents a harmonic spherical wave.

$$
p(r, t)=\frac{j \omega \rho_{0}}{4 \pi r} \hat{Q} e^{j(\omega t-k r)}
$$

The particle velocity is

$$
v_{r}=\frac{p}{\rho_{0} v}(1+1 / j k r)
$$

For distances which are large compared to the wavelength, $k r \gg 1$ the ratio $p / v_{r}$ tends asymptotically to the characteristic impedance of the medium, $\rho_{0} v$. 


\subsection{Characterization of diffuse field}

An acoustic field is considered to be perfectly diffuse in a volume $V$ if the energy density is same on all points in the volume $V$ [4]. In other words, a diffuse field has a uniform sound pressure throughout the room considered. Characterization of diffuse fields in a reverberant room has been an active research problem for a long time. A model to characterize the diffuse field in a rectangular cavity is discussed in [42]. The authors use pressure correlation and spatial uniformity measures to characterize the degree of diffuseness. The authors conclude from the studies that a diffuse field can be established in rooms with strong modal behaviour above what is known as the Schroeder frequency [43]. The general applicability of diffuse field theory with respect to room shape, surface reflections and fittings is discussed in [44]. Effect of each of the parameter is analyzed and the author provides approximate values of certain room acoustic parameters for which a diffuse field in a room can be expected.

The assumption of a diffuse field in a non-diffuse case can affect the accuracy of acoustic applications, especially due to erroneous steady state sound pressure levels. Thus, a method applicable in a general case, such as the image source method is applied to calculate the room acoustic properties if applicability of diffuse field is not certain. The image source method is discussed in detail in the next section.

\subsection{Image Source Method}

The image source method [45] is one of the most important methods to calculate room acoustic properties like reverberation and critical distance. The method helps in finding the point to point room transfer function (RTF) by using the images contributing to the impulse response. The authors claim that the image solution of a rectangular enclosure approaches an exact solution when the walls become rigid. Pressure emitted from a point source at a single frequency $\omega$ in free space is of the form

$$
P\left(\omega, X, X^{\prime}\right)=\frac{e^{j \omega(D / v-t)}}{4 \pi D}
$$

where $X$ is the source, $X^{\prime}$ is the receiver, $D=\left|X-X^{\prime}\right|, v$ is the velocity of sound and $t$ is time. In the case of the presence of a rigid wall, the boundary condition 


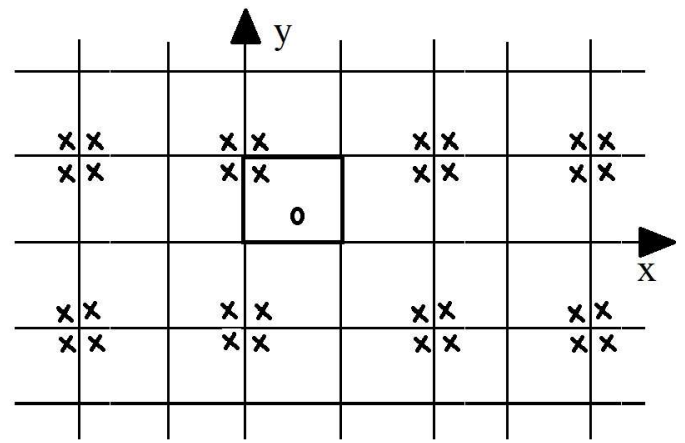

Figure 2.1: Image Expansion for a rectangular room with six walls. 'x' denotes the images considered and 'o' the centre of the room. Source: [45].

(The vertically displaced images do not appear in this two dimensional representation.)

is satisfied by placing an image symmetrically on the far side of the wall. Thus,

$$
P\left(\omega, X, X^{\prime}\right)=\left(\frac{e^{j k D_{+}}}{4 \pi D_{+}}+\frac{e^{j k D_{-}}}{4 \pi D_{-}}\right) e^{-j \omega t}
$$

where $k$ is the wave number given by $\omega / v, D_{+}$is the distance from the microphone to the source and $D_{-}$is the distance from the microphone to the image. These distances are defined by

$$
\begin{aligned}
& D_{-}^{2}=\left(x-x^{\prime}\right)^{2}+\left(y-y^{\prime}\right)^{2}+\left(z-z^{\prime}\right)^{2} \\
& D_{+}^{2}=\left(x+x^{\prime}\right)^{2}+\left(y-y^{\prime}\right)^{2}+\left(z-z^{\prime}\right)^{2}
\end{aligned}
$$

In the case of a rectangular enclosure with 6 walls, image expansion is as shown in Figure. 2.1 which could be written as

$$
P\left(\omega, X, X^{\prime}\right)=\sum_{b=1}^{8} \sum_{a=-\infty}^{\infty} \frac{e^{j k\left|R_{a}+R_{b}\right|}}{4 \pi\left|R_{a}+R_{b}\right|} e^{-j \omega t}
$$

where $R_{b}=\left(x \pm x^{\prime}, y \pm y^{\prime}, z \pm z^{\prime}\right), a$ is the integer vector triplet $(d, e, f)$ and $R_{a}=2\left(d L_{x}, e L_{y}, f L_{z}\right)$ with $\left(L_{x}, L_{y}, L_{z}\right)$ as the room dimensions. Considering the Fourier transform of (2.12), one can write

$$
p\left(t, X, X^{\prime}\right)=\sum_{b=1}^{8} \sum_{a=-\infty}^{\infty} \frac{\delta\left(t-\left|R_{a}+R_{b}\right| / v\right)}{4 \pi\left|R_{a}+R_{b}\right|}
$$


using the time domain Green's function. In the case of non-rigid walls, the model becomes more complicated with the pressure reflection coefficients of the six boundary planes becoming complex. The authors use this model to calculate reverberation time and the critical distances, and conclude that the method is quite efficient.

Other methods of simulating room acoustics include the boundary element method and the finite element method, though these are not pursued in this thesis.

\subsection{Early reflections}

The RTFs measured or evaluated using the image source method can be divided into the direct path, early reflections and the late reverberations. Early reflections in the RTF contributes to speech intelligibility, which is discussed in [13]. The authors use a performance metric, known as Early Reflection Benefit (ERB) which is the difference in decibels between the energies of the early reverberant speech and the direct sound. The speech signal arriving up to $50 \mathrm{~ms}$ after the direct sound is considered to be from the early reflections. Similarly, the direct sound is expected to arrive within the first $10 \mathrm{~ms}$. Therefore, ERB is calculated as the difference in energies in decibels between the speech samples arriving in these two time frames. When speech tests are performed with fixed speaker head positions in both direct and reverberant fields, it is the Signal to Noise Ratio (SNR) that affects the test score. (For example, SNR reduction reduces the test score). On the other hand, in the case of varying speaker head positions, it is the early reflections that affect the test score. The tests proved that increased energy in early reflections has a similar effect on listener intelligibility to that of increased direct energy. Thus they prove that early reflections are useful and conclude that in any design, for speech intelligibility, the focus should first be to maximize the early reflections, and other criteria such as reduction of reverberation time, reduction of late reflections etc are less important.

\subsection{Room response variations}

The RTFs may vary due to atmospheric factors such as temperature and humidity, and changes in position inside the room. An understanding of the fluctuations in RTF due to some of the effects such as changes in microphone position is essential for the design of a robust shaping filter, which is the problem dealt with in this 
thesis. One important paper related to this topic is by Radlovic et al. [38] which describes a theoretical framework to analyze robustness of sound equalization to change of microphone and source positions in a room. The authors derive a model for the mean square error in estimation due to such a change in position. The main assumptions behind this model are

- The linear dimensions of the room are large relative to wavelength,

- The average spacing of the resonance frequencies are smaller than one-third of their bandwidth. (This criterion can be met for all frequencies higher than the Schroeder frequency, $f=2000 \sqrt{T_{60} / V}$ Hz where $T_{60}$ is the reverberation time and $V$ is the room volume.)

- Both source and microphone are in the interior of the room, at least half a wavelength from the walls.

The mean square error due to the displacement of the receiving point at a frequency $f$ is of the form

$$
W(f)=E\left\{|\tilde{C}(f) H(f)-1|^{2}\right\}
$$

where $\tilde{C(f)}$ is the frequency response between the source and the receiver at a distance from the equalization point and $H(f)$ is the frequency response of an inverse filter. The expectation is taken with respect to both the distribution of source locations and distribution of microphones. The source locations are assumed to be uniform throughout the room and at least half a wavelength away from the walls. The microphones are assumed to be uniformly distributed over a sphere centered at the reference location. Let $D$ denote the distance from the source to the reference location and $d$ the displacement from the equalization point, $k$ is the wave number. Now (2.14) can be computed as

$$
W(f) \cong \frac{\gamma \frac{D}{2 d} \ln \left|\frac{D+d}{D-d}\right|+1}{\gamma+1}-2 \frac{\sin (k d)}{k d}+1
$$

where the first term is due to the direct component and the other two terms are due to reverberations. Here, $\gamma$ is approximately equal to the ratio of direct to reverberant power given by

$$
\gamma \simeq \frac{P_{\mathrm{d}}(f)}{P_{\mathrm{r}}(f)}
$$


If displacement from the equalization point is small compared to the source to microphone distance, the first term in (2.15) approaches one, resulting in the inference that the direct field component has a negligible effect on the error signal in such a situation. Thus, the error in the case of only diffuse field is of the form

$$
W(f) \cong 2-2 \frac{\sin (k d)}{k d}
$$

The authors in [38] also derive expressions for the mean square error due to inexact inversion of the room responses, which shows that an inexact inverse filtering results in more errors at a distance from the equalization point especially at high frequencies. This is due to the increased sensitivity of inverse filtering to position changes at high frequencies.

The sections to follow gives an overview of some key research problems in acoustics.

\subsection{Sound field reproduction}

Sound field reproduction is the acoustic problem of generating a desired sound field over a wide area. An approach to the reproduction of plane wave sound fields is discussed in [46]. The design approach is developed based on spherical harmonic decomposition of the wave field to design loudspeaker array weights, positions and order required for good performance.

Sound field reproduction is an indispensable process in a surround sound system. Surround sound systems may be two dimensional (2D) or three dimensional (3D). One example of a sound field reproduction system is the ambisonics system $[47,48]$, the original form of which is 2D. Ambisonics systems, being based upon a spatial decomposition of a sound field, make use of panning functions to determine the loudspeaker weights to achieve the desired sound field [48, 49]. Sound field reproduction performance is affected by a number of parameters such as loudspeaker matching and high frequency interference effects of the panning functions above the spatial Nyquist frequency [46], especially for irregular loudspeaker array layouts. A robust approach for a non-uniform loudspeaker layout is discussed in [50] in which the author uses a least square pressure matching approach to design robust panning functions. The method takes into account the reduction in size of regions of accurate sound reproduction for a virtual sound source between the 
loudspeakers with a large inter-speaker angle. Robustness is achieved by imposing a large penalty weight for loudspeakers far from the intended virtual source angle. The method has advantages of producing a direct solution without any non-linear optimization, applicability to arbitrary layouts and easy adjustment of the trade off between robustness and reproduction accuracy by tuning a single parameter. $3 \mathrm{D}$ surround sound systems offer potential for more accurate reconstruction than 2D systems. There are several methods for 3D sound field reproduction, the most common being wave field synthesis (WFS) [51], inverse method [52] and 3D ambisonics methods [53]. Wave Field Synthesis method is based on the knowledge of pressure and normal velocity on the surface of the reproduction region. The inverse method aims to create the desired sound pressure at a set of discrete points using an inverse matrix created based on the geometry of loudspeakers and receiver positions. The 3D ambisonics method is based on a spherical harmonic decomposition. A 3D ambisonics sound system based on 3D ambisonics method of sound reproduction is discussed in [54]. The spherical harmonic description is employed in this work to achieve the desired sound field reproduction using either a mode matching approach or a simple source approach. The mode matching approach develops a solution for the loudspeaker weights in the form of a pseudoinverse. The simple source approach assumes the reproduction system to be composed of a continuous spherical distribution of monopole sources at a particular radius and develops a solution for loudspeaker weights without the need to calculate the inverse matrix. In [54], the unregularized mode matching approach gave better performance at lower values of $k r$ while the simple source field model performed better (than the unregularized mode matching approach) at higher values of $k r$. The authors in [54] also discuss in detail some recording methods such as free field sphere decomposition, solid sphere decomposition and general array sampling along with their sampling requirements.

A framework for sound field control using a limited number of loudspeakers is proposed in [55]. The system relies on an optimal feed forward controller which is a filter matrix designed based on an estimate of the impulse responses at the measurement position and the target responses at these positions. The system also has a post-processing step to achieve spectral flatness. Authors use both objective and subjective evaluations to validate the system. This framework is based upon a powerful robust linear-quadratic control method $[56,57]$. 


\subsection{Creation of sound zones}

A research problem related to sound field reproduction is the creation of sound zones for multi-zone surround sound. Some optimization approaches focus on the separation and isolation of adjacent bright sound zones [58-60]. In [58], the authors describe a method to control sound in each region, and also to control the leakage into the surrounding regions. They formulate an $l_{2}$ problem with constraints on the total energy and leakage into the quiet zones. The problem is of the form

$$
\begin{array}{ll}
\min _{h} & \left\|H_{\mathrm{c}} h-p_{\mathrm{d}}\right\|^{2} \\
\text { subject to } & \|h\|^{2} \leq K_{0} \\
& \left\|H_{n} h\right\|^{2} \leq K_{n} \quad n=2 \ldots N
\end{array}
$$

where $H_{n}$ describes the acoustic transfer functions from each loudspeaker to $M_{n}$ points inside a zone $D_{n}$ and $K_{n}$ is defined as

$$
K_{n}=\alpha \frac{M_{\mathrm{c}}\left\|p_{\mathrm{d}}\right\|^{2}}{M_{n}}
$$

where $\alpha$ is the acceptable level of sound energy leakage into another listening zone. The Wolfe dual for problem in (2.18) is derived and solved iteratively using an interior point algorithm. The results obtained are compared with those from the standard LS approach and the weighted LS approach using mean square energy, LS weight energy and sound energy leakage as the evaluation criteria.

Closely related to multi-zone surround sound is acoustic contrast control [59, 60]. This problem involves the maximization of a cost function known as acoustic contrast. An example measure [59,60] is given by

$$
A_{c}=\frac{p_{\mathrm{B}}^{H} p_{\mathrm{B}}}{p_{\mathrm{D}}^{H} p_{\mathrm{D}}}
$$

where $p_{\mathrm{B}}$ and $p_{\mathrm{D}}$ are the acoustic pressures in the bright and dark zones respectively. The filter coefficients can be obtained from the generalized eigenvector corresponding to the maximum eigenvalue. Acoustic contrast control can be used for the generation of independent bright zones in a room [60]. One application of this is the control of sound intensity from the adjacent seat, when two people in adjacent seats of an aircraft are listening to different channels [59]. 
A related problem is that of spatial pattern control for reproducing a particular spatial directivity pattern with a typical loudspeaker array. Authors in [61] discuss three methods for achieving spatial pattern control namely Least Squares (LS) approach, maximum energy array and eigenfilter solution based on Total Least Squares (TLS). All the three approaches formulate the problem as a quadratically constrained quadratic programme (QCQP) though the objective is slightly different in each case. The LS approach minimizes the least square error between the generated and the desired spatial pattern. This technique introduces a regularization parameter that controls the performance of the approach. The maximum energy array approach maximizes a cost function based on acoustic contrast to design the filters. A total least squares minimization of the total least squared error between the obtained and desired patterns are used in the eigenfilter design approach. This method has the advantage over other approaches that it does not require calculation of the inversion of matrices. Each of the three approaches are evaluated for their performance and the authors conclude that the maximum energy array approach gives best performance, especially at low frequencies. The performance of the other two approaches depend on the proper choice of a regularization parameter.

\subsection{Evaluation of Direct to Reverberation Ratio}

A measure known as direct to reverberant energy ratio (DRR) is very useful for characterizing the acoustics of a room. An approach to estimate this quantity is discussed in [62]. The authors estimate the DRR by splitting the acoustic energy (specifically the transfer function from the source to the microphones in a reverberant room) into two components which are the direct and the reverberant components. The spatial correlation matrix of the signal received at all elements of a microphone array can be approximately represented by the equation

$$
\left[\begin{array}{cc}
1 & 1 \\
\phi_{12} & \psi_{12} \\
\vdots & \vdots \\
\phi_{1 M} & \psi_{12} \\
\phi_{21} & \psi_{21} \\
1 & 1 \\
\vdots & \vdots \\
1 & 1
\end{array}\right]_{M^{2} \times 2}\left[\begin{array}{c}
P_{\mathrm{D} C}(\omega) \\
P_{\mathrm{R} C}(\omega)
\end{array}\right]_{2 \times 1}=\left[\begin{array}{c}
R_{\operatorname{corr}_{11}}(\omega) \\
R_{\operatorname{corr}_{12}}(\omega) \\
\vdots \\
R_{\operatorname{corr}_{1 M}}(\omega) \\
R_{\operatorname{corr}_{21}}(\omega) \\
\vdots \\
\vdots \\
R_{\operatorname{corr}_{M M}}(\omega)
\end{array}\right]_{M^{2} \times 1}
$$


where $R_{\operatorname{corr}_{p q}}$ are the correlation coefficients between the microphones $p$ and $q, \phi_{p q}$ is a phase factor corresponding to the distance between microphones $p$ and $q$ and $\psi_{p q}$ is a sinc function varying according to the distance between microphones at $p$ and $q$. The spatial correlation of direct path is expressed by a simple phase difference because of the time difference of arrival between the microphones. Assuming the scattering to be isotropic, the correlation of the reverberation component can be approximated by the spatial correlation of a diffuse field i.e., a sinc function. $P_{\mathrm{D} C}(\omega)$ is the power spectrum of the direct component and $P_{\mathrm{RC}}(\omega)$ is the power spectrum of the reverberation components.

Let $\widehat{P}(\omega)=\left[\begin{array}{ll}P_{\mathrm{D} C}(\omega) & P_{\mathrm{R} C}(\omega)\end{array}\right]^{\mathrm{T}}$ and $\widetilde{R}_{\text {corr }}(\omega)=\left[R_{\operatorname{corr}_{11}}(\omega) \ldots R_{\operatorname{corr}_{M M}}(\omega)\right]^{\mathrm{T}}$. Now, equation $(2.21)$ can be written as

$$
F(\omega) \widehat{P}(\omega)=\widetilde{R}_{\text {corr }}(\omega)
$$

the LS solution of which is of the form

$$
\widehat{P}(\omega)=F^{\dagger}(\omega) \widetilde{R}_{\text {corr }}(\omega)
$$

where ${ }^{\dagger}$ represents a pseudoinverse. Here, $F(\omega)$ is known and $R_{\text {corr }}(\omega)$ can be calculated from observed signals. Therefore, each energy component of $\widehat{P}(\omega)$ can be found separately. The authors then use these estimates to find the ratio of direct to reverberant power spectra as

$$
\mathrm{DRR}_{\mathrm{est}}=10 \log _{10} \frac{\sum_{\omega} \widehat{P}_{\mathrm{D} C}(\omega)}{\sum_{\omega} \widehat{P}_{\mathrm{R} C}(\omega)}
$$

The authors also analyze the effect of reverberation time, early reflections, noise, frame length and number of microphones on the accuracy of estimation.

\subsection{Modelling of Head Related Transfer Func- tions}

Modeling of Head Related Transfer Functions (HRTFs) plays an important role in the study of spatial hearing. The authors of [63] present an approach that use an image method to compute the spherical harmonic coefficients and use these coefficients to describe the effect of a wall of a room on the HRTF based on this model. The dependence of HRTFs on torso and pinnae are modeled in [64], using the KEMAR mannequin and a snowman model. The authors use a combination of different methodologies, such as acoustic measurements for the KEMAR mannequin to 
validate the results from the numerical methods, a multi-pole re-expansion method for spherical torso in the snowman model and a boundary element method for an ellipsoid torso and conclude that their method is a good approximation to find the effects of torso and pinnae on the HRTF.

\subsection{Chapter Summary}

The literature reviewed here gave an overview of some basic acoustic principles and common research problems in acoustics. The following chapter discusses some literature related to the impulse response shaping formulation, which has applications in acoustic problems as discussed in this chapter. 


\section{Chapter 3}

\section{Literature review II \\ (Shortening formulations and Optimization Algorithms)}

In this thesis, acoustic room impulse response shaping is formulated as an optimization problem that minimizes a cost function formulated on the required criteria to solve for the pre-filters. Some key literature related to this is detailed in this chapter.

\subsection{Impulse Response Inversion}

An understanding of the basic theory of inverse filtering and its formulation is essential for appreciating the concept of impulse response shaping. Channel inversion is a well known technique that helps to retrieve a signal travelling through a medium by compensating for the effects of the medium. This is achieved using an inverse filter. In the case of acoustic signals propagating inside a room, there can be various distortions such as reflection from the walls, floor and ceiling which result in unwanted reverberations in the signal. In order to retrieve the signal at the listening locations, these reverberations in the room impulse response (RIR) can be removed or compensated for by using a pre-filter. One of the early papers of acoustic channel inversion, Miyoshi et al. [18] assumes that the distortions of acoustic signals radiated inside a room due to wall reflections are linear and discuss an inverse filtering scheme to overcome the effects of the room impulse response. They present two approaches, one of which is the conventional inverse filtering scheme using a Moore Penrose pseudoinverse derived on a least square error criterion and the other is based on a multi-channel realization that allow the use of a matrix inverse instead of a pseudoinverse. The authors also derive the conditions under which the multi-channel inverse filters yield a feasible solution. 
If a filter $h(n)$ is an inverse of the system response $c(n)$, then the combined response given by $r(n)=c(n) * h(n)$ is a delta function that represents the resultant desired response. This can be written in matrix format as

$$
r=C h
$$

where $r \in \mathbb{R}^{N_{\mathrm{c}}+N_{\mathrm{h}}-1}, h \in \mathbb{R}^{N_{\mathrm{h}}}$ and $C$ is an $N_{\mathrm{c}}+N_{\mathrm{h}}-1 \times N_{\mathrm{h}}$ Toeplitz matrix representing convolution. The Least Squares(LS) solution for the problem in (3.1) can be found by

$$
\min _{h} J_{c}=\|r-C h\|_{2}^{2}
$$

the solution of which is of the form

$$
h=\left(C^{T} C\right)^{-1} C^{T} r=C^{\dagger} r
$$

This scheme can be extended to multiple-input multiple-output linear Finite Impulse Response (FIR) systems. The authors of [18] prove that their proposed method for estimating inverse filters outperforms the conventional method through simulations by using estimation error as a performance metric.

In order to reduce the effect of noise and measurement errors, a regularized least square problem can be formulated as in [22,65-68]. In spite of this, the greatest challenge with using inversion for acoustic equalisation is that the room impulse responses, which are compensated by inversion, vary with room geometry, temperature and also with positions inside the room. Hence channel inversion may not be effective unless the channel estimate is updated frequently.

\subsection{Impulse Response Shaping (or Shortening)}

Acoustic equalization can be accomplished more efficiently than channel inversion by using impulse response shortening. In personal audio, for example, the aim is to create independent sound zones within the same listening space. This problem is challenging because of the sound leakage from one zone to another, otherwise known as cross-talk. Loudspeakers with appropriate directivities can be located near to the listeners and sound absorption can be introduced between sound zones to improve sound isolation $[69,70]$. However, performance can be improved further by pre-processing the loudspeaker signals with cross-talk cancelation filters 
(CCFs). Using impulse response shaping, the resultant or global impulse response (GIR), i.e., the combination of both a pre-filter and an acoustic impulse response, can be shortened or made to possess more desirable properties than that of the original impulse response. Thus, shortening (or shaping) techniques offer advantages over inversion by imposing less stringent criteria and allowing some of the perceptually useful features such as early reflections whilst reducing the undesirable late reverberation. An algorithm for determining CCFs using a flexible impulse response reshaping approach that outperforms traditional inverse-filter designs is discussed in [27]. The algorithm uses an interior point method to find the shaping filters.

Impulse response shortening was first applied to the problem of equalizing the reverberant channel between a loudspeaker and a microphone by Kallinger et al. [19]. The principal difference between the applications in communication and acoustics is that in the latter the psychoacoustics of the human listener must be taken into account. In [19], a least squares (LS) impulse shortening approach with the generalized eigenvector solution of [23] was proposed. This method minimizes the ratio of the energies of the undesired and desired parts of an RIR in accordance with the D50 measure of speech intelligibility. D50 is defined as the ratio of the energy within $50 \mathrm{~ms}$ after the first peak of the RIR to the total energy of the RIR. Similarly Mei et al. [71] devised a reshaping approach based upon an LS objective function with a linear constraint to guarantee energy in the desired part. This approach was compared to the approach of [19] as well as an eigenvector approach minimizing the undesired energy subject to a norm constraint on the desired filter.

In general, acoustic channel inversion is sensitive to additive noise and channel estimation error. In this context, channel shortening algorithms have been shown to be less sensitive than the inversion techniques. Based on the multipleinput/output inverse theorem (MINT) [18], a relaxed multi-channel least squares (RMCLS) approach of channel shortening was proposed, which is applicable for dereverberating the signal of a source using multiple microphones [65, 72, 73]. Here, the link was explored between RMCLS and the MINT approach, establishing the MINT solution as a special case.

Thomas et al. [22] investigated impulse shortening with RMCLS to show that shortening the channel to several milliseconds provides a more robust solution than ideal channel equalization. The enhancement comes from a reduction in the en- 
ergy of designed filters (due to reduction in $\ell_{2}$ norm). In a related approach called partial multi-channel equalization using MINT or P-MINT [66], an exact solution to the underdetermined shortening problem is obtained by setting several taps to be same as those in one of the RIRs [68]. This P-MINT approach was shown to be superior in performance to channel shortening and similar in performance to RMCLS. These approaches do not require concentrating energy into the early reflections of the GIRs, so that filters can be designed with less energy. Further improvements to filter robustness have been obtained with Tikhonov regularization [68] which can further reduce filter energy but at the expense of performance. Here, the L-curve method of choosing the regularization parameter [74] was shown to approximately optimize the perceptual speech quality. P-MINT is a specific solution to the channel shortening problem, for the case that the problem is underdetermined and hence can be solved exactly [68].

However, the $\ell_{2}$ norm approaches were seen to produce late reverberant echoes as well as undesirable frequency domain distortions in the GIRs. More desirable time domain properties are obtained using an $\ell_{\infty}$ norm [20] which guarantees a steady decay of the GIR free from any strong late-reverberant echoes. An $\ell_{\infty}$ approach was solved in [20] using steepest descent methods. The approach was further extended to impulse response reshaping using a more flexible $p$-norm objective function [21] that allows more rapid convergence than [21]. This criteria, like the minimax criteria, was shown to ensure better temporal qualities than the $\ell_{2}$ norm. Included also in the approach of [21] was an auditory mask, a temporal decay curve below which the reverberation in an impulse response is considered to be imperceptible [75]. The optimization problem solved in [21] is of the form

$$
\min _{h} f(h)=\log \left(\frac{f_{\mathrm{u}}(h)}{f_{\mathrm{d}}(h)}\right)
$$

where $f_{\mathrm{d}}(h)=\left\|g_{\mathrm{d}}\right\|_{p_{\mathrm{d}}}$ and $f_{\mathrm{u}}(h)=\left\|g_{\mathrm{u}}\right\|_{p_{\mathrm{u}}}$. The vectors $g_{\mathrm{d}}$ and $g_{\mathrm{u}}$ are obtained by stacking all the wanted and unwanted portions of the GIR, $p_{\mathrm{d}}$ and $p_{\mathrm{u}}$ are the corresponding norm types. Here, $g_{\mathrm{d}}=w_{\mathrm{d}} \cdot g$ and $g_{\mathrm{u}}=w_{\mathrm{u}} \cdot g$ where $w_{\mathrm{d}}$ and $w_{\mathrm{u}}$ are the weight vectors corresponding to the desired and undesired parts respectively and $\cdot$ is the element-wise product. The weight vector specifies the desired shape of the GIR.

A basic drawback of reshaping approaches is the large amount of computation required to obtain the CCFs. This is mainly because these techniques need to deal 
with long room impulse responses. Hence, it takes a large amount of computation time to converge to a solution when gradient descent or interior point methods are used. The steepest descent approach is commonly used, where the convergence rates are only linear. The minimax approach of [20] converges even more slowly as each iteration only modifies a single tap of the shortening filter. A modification to the steepest descent update rule was made that allowed the algorithm to converge faster and be less likely trapped in a local minima [32]. This modification was based upon a diagonal approximation of the Hessian matrix, used in the steepest descent iteration.

Jungmann et al. [76] extended the minimax impulse response shortening approach of [20] to perform combined cross-talk cancelation and listening room compensation. Impulse response reshaping was used to simultaneously cancel the cross-talk and apply the auditory mask. The optimization formulation is similar to (3.4), the weight vector applied is derived based on the auditory behaviour of human ear [3].

The weight vector for the desired part of the direct responses is defined as

$$
w_{\mathrm{d}}(n)= \begin{cases}1 & t_{0 m} \cdot F_{\mathrm{s}} \leq n \leq\left(t_{0 m}+T_{d}\right) F_{\mathrm{s}} \\ 0 & \text { otherwise }\end{cases}
$$

where $F_{\mathrm{s}}$ is the sampling frequency, $t_{0 m}$ is the average time taken from the $L$ loudspeakers to the microphone $m$ and $T_{d}$ is chosen to be $4 \mathrm{~ms}$. The weight vector for the undesired portion of the direct response is defined based on the acoustic masking limit $[77,78]$ as

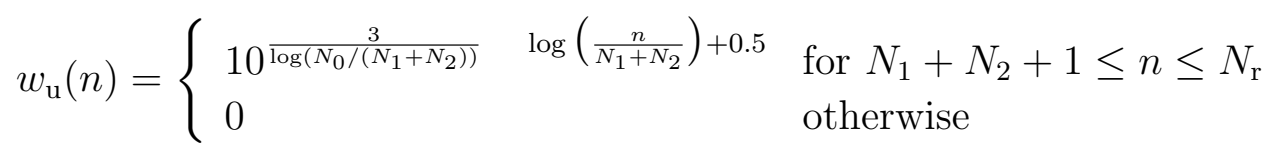

where $N_{1}=t_{0 m} \cdot F_{s}, N_{2}=T_{d} \cdot F_{s}$ and $N_{0}=0.2 F_{\mathrm{s}}+N_{1} \cdot N_{\mathrm{r}}$ is the length of the GIR. The weight vector on the undesired cross-talk path can then be used to set the desired cross-talk cancelation.

A minimax formulation (or minimization of infinity norms) of the shaping problem using a relaxed multi-channel approach is discussed in [27,31], which is then solved using the epigraph form [41]. The minimax optimization formulation to solve the shortening problem is

$$
\min _{h} J_{c}(h)=\|W(r-C h)\|_{\infty}
$$


where $W$ is a diagonal weighting matrix. (3.7) is converted to an $\ell_{1}$ norm problem using the epigraph form as

$$
\begin{array}{ll}
\min _{a, h} & a \\
\text { subject to } & {[W(C h-r)]_{n} \leq a} \\
& {[W(C h-r)]_{n} \geq-a}
\end{array}
$$

where $C$ is the convolution matrix representing the loudspeaker to microphone channel responses, $h$ is the collection of impulse responses of the filters to be designed, $W$ is a weighting matrix and $r$ is the vector that contains the GIRs $r_{m s}$ each of which represents the convolution of channel responses and inverse filters i.e.,

$$
r_{m s}=\sum_{l=1}^{L} C_{m l} h_{l s}
$$

The desired responses are

$$
\begin{gathered}
r_{m s}=0_{N_{\mathrm{r}}}, m \neq s \\
r_{s s}=\left[0_{N_{\mathrm{mp}}}^{T}, 1,0_{N_{\mathrm{eq}}}^{T}\right]^{T}
\end{gathered}
$$

where $N_{\text {mp }}$ includes the length of the response up to the peak and $N_{\text {eq }}$ exceeds the length of the reverberation of the response.

The weighting function is defined as

$$
w_{s s}=\left[1_{N_{\mathrm{r} 1}}^{T}, w_{\mathrm{d}}^{T}, w_{\mathrm{u}}^{T}\right]^{T}
$$

where $N_{\mathrm{r} 1}=N_{\mathrm{mp}}+1, w_{\mathrm{d}}$ penalizes early reverberation and $w_{\mathrm{u}}$ defined as

$$
\left[w_{\mathrm{u}}\right]_{n}=e^{\beta\left(n-N_{\mathrm{r} 3}\right) / N_{\mathrm{r} 3}}
$$

penalizes late reverberation. $N_{\mathrm{r} 3}$ is the length of the late reverberations and $\beta$ decides the rate at which late reverberations are penalized. $w_{\mathrm{d}}$ is the weight assigned to the desired part of the response.

The formulation developed in [31] based on HRTF is very similar to (3.7), which is solved in its dual using conic programming. The performance metrics used are minimum channel separation, maximum cross-talk and maximum deviations of direct responses from $0 \mathrm{~dB}$ as the evaluation criteria. 
The convex problem in (3.8) is solved iteratively using interior point methods in [27]. In contrast, the problems in $[21,23,39]$ are non-convex and may lead to suboptimal solutions due to local minima. When solved using interior point methods, rapid quadratic convergence rates are guaranteed in a small number of iterations. However, a Hessian matrix must be formed, the dimensions of which are large, being proportional to the number of taps $N_{\mathrm{c}}$ in the RIRs. Hence, the number of entries in the matrix varies with $N_{\mathrm{c}}$. This highlights the importance of not only fast converging algorithms to solve for the shaping filters but ones which are also memory efficient.

The formulation of the shaping problem as a regularised minimization allows the use of some fast iterative algorithms for solving the optimization problem to find the shaping filters. One such fast algorithm is the Dual Augmented Lagrangian Method (DALM) [34-36]. The DALM algorithm has been originally proposed to provide a sparse solution for the $\ell_{1}$ regularised $\ell_{2}$ minimizations (D-2-1) as in the Basis Pursuit DeNoising (BPDN) [37] problems that arise in sparse reconstruction. The steepest descent approaches in $[20,21,32]$ choose a suitably varying step size parameter, which requires a line search algorithm [41, p. 464-466]. Even then an unacceptable number of iterations may be required for convergence, especially for very large problems which are usually poorly conditioned. The computationally fast solution methods like DALM can overcome these issues. The DALM algorithm converges fast even for large problems due to the addition of an augmentation term in the dual Lagrangian.

\subsection{Perceptually enhanced shaping solutions}

Shaping approaches that are perceptually enhanced and less sensitive to noise are discussed in $[14,79]$. The authors of [79] use a regularized approach to design filters that are less sensitive to RTF fluctuations and noise. They use a cost function of the form

$$
J=\|C h-r\|^{2}+\delta\|h\|^{2}
$$

where the second term is an addition to the LS cost function to capture the effect of disturbances. The solution is of the form

$$
h=\left(C^{T} C+\delta I\right)^{-1} C^{T} r
$$

where $C$ is the mean RTF in case of fluctuations. In the presence of noise, $I$ is replaced by $R_{\mathrm{n}}$, the noise correlation matrix and $C$ is the RTF. The authors 
discuss the factors affecting filter design which are filter length, modeling delay and the value of the regularization parameter, and analyze their effect on design through analysis of simulation results using Signal to Distortion ratio as an evaluation criterion. They infer from the studies that the proper choice of regularization parameter is a more crucial design parameter than modeling delay and filter length.

Kodrasi et al. [14] discusses a perceptually constrained approach of impulse response shortening method that preserves speech quality and also results in higher reverberant tail suppression than channel shortening or the regularized partial multi-channel equalization technique based on the Multiple-Input/Output Inverse Theorem (P-MINT). This approach is computationally more expensive and time consuming than the previous approaches. The optimization problem in [14] is a maximization of the Rayleigh quotient.

$$
J_{\mathrm{C}}=\frac{\left\|W_{\mathrm{d}} \hat{C} h\right\|_{2}^{2}}{\left\|W_{\mathrm{u}} \hat{C} h\right\|_{2}^{2}}=\frac{h^{T} \hat{B} h}{h^{T} \hat{A} h}
$$

where $\hat{C}$ represents the estimated channel response between the loudspeakers and the microphones, $h$ is the inverse filter to be designed, $W_{d}=\operatorname{diag}\left(w_{\mathrm{d}}\right), W_{u}=$ $\operatorname{diag}\left(w_{\mathrm{u}}\right)$ and

$$
\begin{aligned}
& \hat{B} \triangleq \hat{C}^{T} W_{\mathrm{d}}^{T} W_{\mathrm{d}} \hat{C} \\
& \hat{A} \triangleq \hat{C}^{T} W_{\mathrm{u}}^{T} W_{\mathrm{u}} \hat{C}
\end{aligned}
$$

The solution can be obtained by solving the generalized eigenvalue problem

$$
\hat{B} h=\lambda_{\max } \hat{A} h
$$

and choosing the largest eigenvector solution. The perceptually enhanced formulation is derived from the above formulation as the maximization of

$$
J_{\mathrm{PeCCS}}(\alpha)=\left\|\hat{C} H_{\mathrm{CS}} \alpha-\hat{r}_{\mathrm{p}}^{\mathrm{d}}\right\|_{2}^{2}+\left\|H_{\mathrm{CS}} \alpha\right\|_{2}^{2}
$$

where $H_{\mathrm{CS}}$ is a matrix containing all the generalized eigenvectors of (3.17), $\alpha$ is a vector of scalar coefficients that also maximizes the Rayleigh quotient in (3.15) and $\hat{r}_{\mathrm{p}}^{\mathrm{d}}$ is the expected response. Their proposed Perceptually enhanced Constrained Channel Shortening (PeCCS) is a two step equalization that involves computation of $\alpha_{\mathrm{PeCCS}}$ as

$$
\alpha_{\mathrm{PeCCS}}=\left[\left(\hat{C} H_{\mathrm{CS}}\right)^{T} \hat{C} H_{\mathrm{CS}}+H_{C S}^{T} H_{\mathrm{CS}}\right]^{-1}\left(\hat{C} H_{\mathrm{CS}}\right)^{T} \hat{r}_{\mathrm{p}}^{\mathrm{d}}
$$


and the computation of filter $h_{\mathrm{PeCCS}}$ as

$$
h_{\mathrm{PeCCS}}=H_{\mathrm{CS}} \alpha_{\mathrm{PeCCS}}
$$

The authors show through simulations that their model preserves speech quality in addition to equalizing the channel. This approach is dependant on the number of taps in the 'don't care' region. (The region that is unaffected by shaping is defined as the 'don't care' region. In the case of a room response, the early reflections that are perceptually useful $[14,66]$, are not shaped and hence constitute the 'don't care' region.)

\subsection{Robust implementation}

Robustness of the shaping filters is an important design criterion because the room responses vary with changes in microphone positions or atmospheric conditions such as temperature and humidity. An approach to achieve robustness to changes in microphone positions is presented in Jungmann et al. [76]. The design was performed over multiple realizations of the channel matrix. This robust approach may add considerable computational overhead which can be reduced by incorporating a statistical sound field model of the perturbations into deriving the CCFs.

A very similar approach [39] generates perturbed channels corresponding to changes in microphone positions and designs shaping filters that are effective for all these channels. The perturbed room impulse response is expressed as a sum of the original RIR and the perturbation caused by changes in microphone positions. The perturbations are modeled as random signals with specific time and frequency properties.

A frequency regularization approach to reduce sharp spectral peaks in the GIRs was proposed in $[28,80]$. This was done by introducing an additional frequency domain penalty term to the objective function used in (3.4). The tall peaks are shown to be penalized in the frequency response using this approach.

Alternatively, RMCLS can be modified slightly to add temporal constraints to avoid spectral coloration [72]. A hybrid approach based on RMCLS and delay-andsum beamforming was devised in [73], to make the solution behave more robustly 
but also with reduced reverberation suppression performance.

A different approach to achieve spatial robustness by utilizing the time domain structure of the room response is presented in [57,81-83]. The authors discuss a LS equalization scheme by probabilistically modelling RTF variability. The approach for a single channel is presented in [81]. An observation used in this work is that the direct path is independent of receiver position and it is the reverberation that is affected by change in position. Accordingly, the authors assume the filters to be composed of two parts: a fixed part corresponding to the direct path and early reflections and a variable part (distributed as a Gaussian IID) corresponding to the reverberation. The algorithm adaptively designs filters based on a feed-forward control scheme using the mean square error (MSE) criterion. The technique achieves good pre-echo cancellation, improved peak-to-tail ratio and good time performance at low frequencies.

The single channel compensation problem in [81] is extended to a MIMO case in [82]. The feed-forward control scheme used by the authors is similar to that in [81] except that some scalars in [81] become vectors. The authors provide results of testing the approach for a varying number of support loudspeakers. The structure of the sound field reproduction set up in [82] has been simplified by considering symmetry in [57]. Similarity points are chosen to simplify the structure and the performance is evaluated by varying the number of similarity points and control points. The performance was shown to increase with more similarity points; but at a cost of lower MSE. The structure in [57] is analysed in terms of the reproducibility of the sound field in [83]. The authors investigated on the choice of the penalty matrix and found that a diagonal weighting matrix is sufficient for the structure in [57] to reproduce the sound field. The authors have also found that Finite Impulse Response filters can be used; but frequency controlled weights may be needed to reduce the peaks and troughs. They also develop a scheme for online adaptation of the reverberant part of the system. The importance of this approach is a powerful framework which can be used to perform partially minimum phase designs.

\subsection{Efficient sub-band implementation}

Computational speed of shortening may be improved if the estimation and shortening are performed in sub-bands. Such an approach is discussed in [84] in which the authors use a typical multi-channel transmission model and design the inverse 
filters in the sub-bands for equalization. The transform used in their transmission model is a generalized DFT (GDFT), which is defined as

$$
u_{m, n}=p_{n} e^{j \frac{2 \pi}{K}\left(m+m_{0}\right)\left(n+n_{0}\right)}
$$

with $m_{0}=1 / 2$ and $n_{0}=0$, where $p_{n}$ is a prototype filter. The inverse filtering operation is then carried out in the sub-band, which results in a significant reduction of computational complexity. They use the LS approach to design each of the sub-band filters, but since the operation is carried out in the sub-band level, it results in the computation of a lower order pseudo inverse due to the presence of a smaller channel matrix.

\subsection{Optimization algorithms}

In the previous sections, some of the optimization formulations used for the shaping problem have been discussed. This section describes the common solution algorithms used for solving such formulations.

\subsubsection{Steepest Descent Algorithm}

Steepest Descent or gradient descent, introduced by Cauchy [85] is a first order iterative optimization algorithm. It can be used to to find a local minimum of a function by taking steps proportional to the negative of the gradient (or of the approximate gradient) of the function at the current point. Choice of the step size parameter is a crucial factor in deciding convergence. Generally, steepest descent algorithm shows a linear convergence rate.

The gradient descent can be combined with a line search to find a locally optimal step size in every iteration. The line search approach finds the direction in which the objective function will be reduced and then computes the step size that decides the amount by which the iteration should move along that direction. The simplest way of performing a line search is by using bisection method that first identifies a range in which the minimum lies and then makes the range smaller and closer to the minimum. This method is very simple since only one extra internal point needs to be calculated in each subsequent step. The most simple and effective direct search method is the golden section search [86], which preserves the interval proportions (golden ratio) regardless of how the search proceeds. A line search method most commonly used with gradient descent is the backtracking line 
search [87] that starts with a relatively large estimate of the step size and iteratively reduce the step size until the objective function is reduced to the expected value. The line search can be time consuming. Conversely, using a fixed small step size can yield poor convergence. Therefore, the most ideal situation for a gradient descent to work well would be the right choice of a suitable step size parameter.

A suitable choice of this parameter is discussed in [88], which even works for large dimensional problems. Many variations to the steepest descent approach have been developed to increase the convergence rate [89-91]. Most of them concentrate on methods to reduce the effect of choice of step size parameter on convergence, such as adaptively changing the parameter to increase convergence rate. The steepest descent algorithm is used for solving the shaping formulation in [21, 28, $32,39]$.

\subsubsection{Interior Point Methods}

Interior point methods are efficient algorithms that can be used to find solution to convex programming problems in polynomial time. The algorithms work by traversing the interior of the feasible region to reach the best solution. The class of primal-dual path-following interior point methods, such as Mehrotra's predictorcorrector algorithm [92], are the best suited for practical implementations. Impulse response shaping approaches presented in Betlehem et al. [27], use interior point method for finding the solution, but are memory exhaustive and computationally slow for practical implementations.

\subsubsection{Conjugate Gradient Descent Algorithm}

The conjugate gradient method (CG) [93] is an algorithm for solving a particular system of linear equations $A x=b$ with symmetric and positive definite matrices. The algorithm is a type of iterative algorithm for solving large system of equations, which arise while solving for partial differential equations or optimization problems. The algorithm progresses by moving in a direction that minimises a metric that decides the closeness to the solution $x^{*}$ that is the unique minimiser of the function

$$
f(x)=\frac{1}{2} x^{\mathrm{T}} A x-x^{\mathrm{T}} b
$$

The algorithm starts by initialising an $x_{0}$ and in each iteration, updating it based on a residual $r_{k}=b-A x_{k}$. The step direction is also made to be orthogonal to 
the previous step directions so that the solution converges quickly. This is an advantage over the steepest descent approach. The problem with the use of steepest descent for poorly conditioned problems (as can happen in the case of shaping formulations) is that the iterations can get trapped in long valleys and thus result in a slow convergence.

It can be seen that the residual $r_{k}$ used in the CG method is the negative gradient of the function $f(x)$ at $x_{k}$; hence the algorithm is called conjugate gradient descent. This algorithm is very fast and has been explored in this thesis work to increase the computational speed of the impulse response shaping problem. Generally, the algorithm converges in $n$ steps if the matrix $A$ has $n$ distinct eigenvalues. In many cases, a pre-conditioner is required to ensure fast convergence of the conjugate gradient method. This algorithm is then called pre-conditioned conjugate gradient (PCG) [94].

\subsubsection{Sparse Reconstruction Algorithms}

In earlier discussions, we mentioned that impulse response shaping approaches based on steepest descent and/or interior point methods are computationally slow and cannot be used for real time implementations. In this section, sparse reconstruction algorithms are explored, which have been found to be computationally very efficient in solving sparse reconstruction problems.

Sparse reconstruction algorithms were developed for solving problems of the form of Basis Pursuit DeNoising (BPDN) [37]. The algorithms can be broadly classified into greedy and non-greedy algorithms. The greedy algorithms include matching pursuit algorithms such as Orthogonal Matching Pursuit (OMP) [95-97]. The algorithm works by finding the best matching projections of multidimensional data onto an overcomplete dictionary. This is done by choosing the column of the overcomplete dictionary that has the largest inner product with the signal, subtracting the approximation of the signal using that column (as a base) from the signal and repeating the process until the norm of the residual is small. The coefficients extracted are updated after each step by computing the orthogonal projection of the signal onto the bases selected so far. These algorithms are computationally expensive pointing to the need of non-greedy algorithms.

The non-greedy algorithms belong to a class of either thresholding algorithms or 
Augmented Lagrangian Methods. One of the well known thresholding algorithms is the Iterative Soft Thresholding Algorithm (ISTA) [98] and its faster versions (FISTA) [34, 99-101]. This algorithm solves the BPDN problem using an iteration that also involves a thresholding step which results in a fast convergence. FISTA algorithms have been widely used in numerous applications for solving sparse reconstruction problems in a computationally efficient manner.

Some of the augmented Lagrangian methods are Primal Dual Augmented Lagrangian (PAL) [102], Dual Augmented Lagrangian Method (DALM) [34-36] and Alternating Direction Method of Multipliers (ADMM) [41]. These methods contain an augmented term in the Lagrangian that accelerates convergence of the algorithm. PAL and ADMM uses an augmentation term in the primal Lagrangian, while DALM uses it in the dual. Both DALM and ADMM work by alternately updating primal and dual variables. This results in fast convergence to the solution. A faster version of DALM called Fast DALM [34] uses some additional steps to further improve the computational efficiency. It uses a combination of DALM iterative steps and a Conjugate Gradient step to achieve this efficiency. This algorithm has been widely used in this thesis to develop a computationally fast shaping algorithm that can be implemented in real time.

\subsection{Chapter Summary}

The chapter examined some of the optimization formulations that are relevant to understand the work presented in this thesis. The chapter started with a discussion of the traditional inverse filtering scheme and its optimization formulation, followed by a more relaxed approach of impulse response shaping. Various shaping formulations available in the literature were presented along with their optimization formulation. Various metrics like maximum cross-talk and direct to reverberant energy ratio can be used for evaluating the performance of such approaches. The chapter also discussed the common solution algorithms, viz., steepest descent and interior point methods, used for solving such formulations. Their disadvantage of low computational efficiency, when applied to the shaping problem, is clear. An overview of the sparse reconstruction algorithms, used in this work, to improve the computational efficiency is also presented. The details are explained in the chapters to follow.

Having reviewed the relevant literature in acoustics and optimization related 
to this work, one can now appreciate the shaping formulation presented in this thesis work and its relevance. The following chapter describes in detail the optimization formulation of the impulse response shaping approach used in this work, including all the parameters and performance metrics used for room compensation and spatial audio problems. 


\section{Chapter 4}

\section{Problem Formulation}

\subsection{Shaping problem}

Impulse response shaping is a pre-filtering technique that can be used to reshape or shorten the impulse responses of channels. This technique is well known in wireless communication as being deployed in discrete multi-tone receivers [23-26] to reduce intersymbol interference. Shaping (or shortening) was first applied to room acoustics to reduce the reverberance by Kallinger et al. [19]. In acoustics, shaping has application in listening room compensation for reducing the perceptible effects of reverberation that distorts the audio signals propagating inside a room. Such a configuration consists of multiple loudspeakers which are mounted inside the room and microphones measuring the responses at specific points. Hence, the problem environment is considered as a multi-channel system, with each channel representing a signal path between a specific loudspeaker and microphone. Each channel response is composed of the reverberations caused by wall and floor reflections as well as reflection from other objects. Thus, the signal received at each microphone is a mixture of distorted signals from multiple loudspeakers. In order to achieve listening room compensation, pre-filters are applied before the loudspeakers to cancel or reduce the effects of reverberation as well as cross-talk. This requires pre-determination of the channels and computation of the pre-filters. The room responses are non-stationary and can vary with time. Therefore, the channel might have changed while the filters are being computed. This can result in poor performance of the system when inverse filtering (equalization) is used. The shaping approach, on the other hand, is more robust to such variations than equalization since it aims to reduce the reverberations and not cancel them. The optimization formulation presented in this chapter aims to achieve joint shaping and cross-talk 


\section{Loudspeakers}

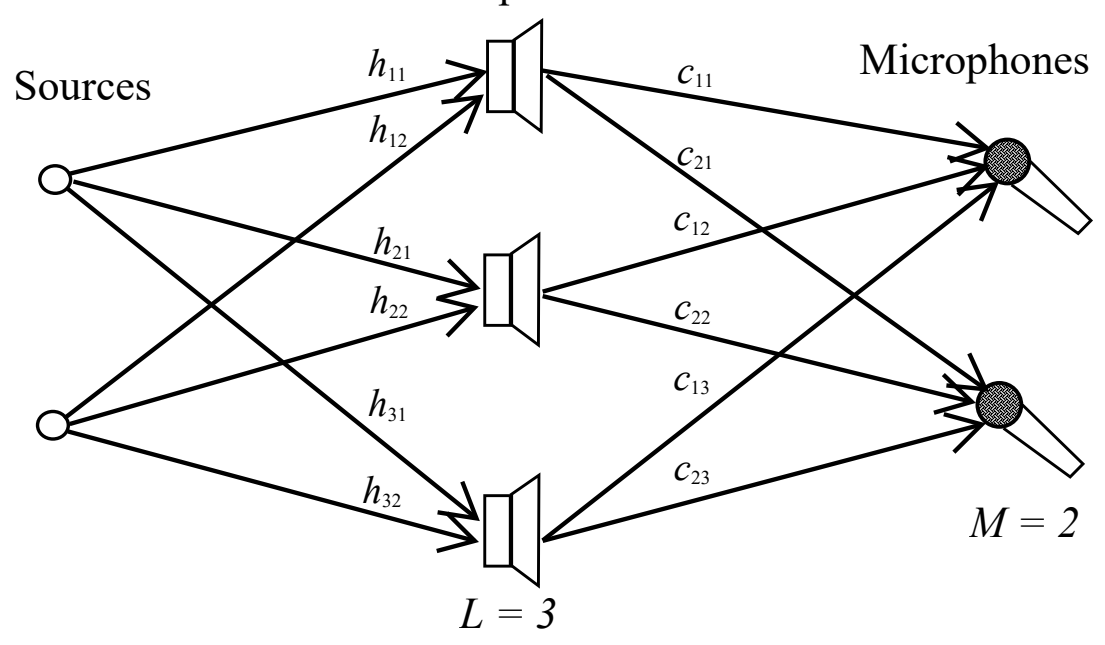

Figure 4.1: A loudspeaker microphone setup showing acoustic channels $c_{m l}$ and cross-talk cancelation filters $h_{l s}$.

cancelation as required in multi-channel listening room compensation.

Consider a typical cross-talk canceler system shown in Figure 4.1. The signals are delivered from $S$ sources to $M=S$ microphones through $L$ loudspeakers. The shaping pre-filters are designed to perform joint shaping and cross-talk cancelation. Here, $c_{m l}$ represents the channel impulse response from loudspeaker $l$ to microphone $m$ and $h_{l s}$ represents the cross-talk canceling pre-filter from source $s$ to loudspeaker $l$. For each path, the combined filter and channel response (called the Global Impulse Response (GIR)) $r_{m s}$ is found [27] as the sum of the convolutions of $c_{m l}$ with $h_{l s}$ over the $L$ loudspeakers. For the purpose of simplifying the presentation, the example case for which $M=S=2$ and $L=3$ is used. For the special case shown in Figure 4.1, a matrix equation for GIRs can be written as $R=\mathcal{C} H$ or

$$
\left[\begin{array}{ll}
r_{11} & r_{21} \\
r_{12} & r_{22}
\end{array}\right]=\mathcal{C}\left[\begin{array}{ll}
h_{11} & h_{12} \\
h_{21} & h_{22} \\
h_{31} & h_{32}
\end{array}\right]
$$


where $\mathcal{C}$ is the matrix defined as

$$
\mathcal{C}=\left[\begin{array}{lll}
C_{11} & C_{12} & C_{13} \\
C_{21} & C_{22} & C_{23}
\end{array}\right]
$$

of dimension $N_{\mathrm{r}} M \times N_{\mathrm{h}} L$ where $N_{\mathrm{r}}$ is the length of each GIR $r_{m s}$ and $N_{\mathrm{h}}$ is the length of each shaping filter $h_{l s}$. The Toeplitz convolution matrices $C_{m l}$ in $\mathcal{C}$, each constructed from the channel impulse responses $c_{m l}$ (of length $N_{\mathrm{c}}$ ) are given by

$$
C_{m l}=\left[\begin{array}{cccc}
c_{m l}(0) & 0 & \cdots & 0 \\
c_{m l}(1) & c_{m l}(0) & \cdots & 0 \\
\vdots & \vdots & \ddots & c_{m l}(0) \\
0 & 0 & \cdots & c_{m l}\left(N_{\mathrm{c}}-1\right)
\end{array}\right]
$$

Using the notations $r=\operatorname{vec}(R), h=\operatorname{vec}(H)$ and $C=I_{2} \otimes \mathcal{C}$ (where $I_{2}$ is the $2 \times 2$ identity matrix, $\otimes$ is the Kronecker product), (4.1) can be rewritten as $r=C h$ or

$$
\left[\begin{array}{l}
r_{11} \\
r_{21} \\
r_{12} \\
r_{22}
\end{array}\right]_{M^{2} N_{\mathrm{r}} \times 1}=\left[\begin{array}{ll}
\mathcal{C} & 0 \\
0 & \mathcal{C}
\end{array}\right]\left[\begin{array}{l}
h_{11} \\
h_{21} \\
h_{31} \\
h_{12} \\
h_{22} \\
h_{32}
\end{array}\right]_{L M N_{\mathrm{h}} \times 1}
$$

The joint shaping and cross-talk cancelation formulation aims to minimize some norm of a weighted error function, given by

$$
\min _{h}\|W(C h-r)\|
$$

where $W=\operatorname{diag}(w)$ is a diagonal weighting matrix of weighting coefficients [27]. The weighting coefficients are chosen so as to heavily penalize the cross-talk $\left(r_{m s}\right.$, $m \neq s)$ as well as late reverberations and pre-echo in the direct channels $\left(r_{m m}\right)$, but only lightly penalize the early reverberations [27].

\subsubsection{Definition of the weight vector}

The joint shaping and cross-talk cancelation problem is formulated as a weighted minimization in which a weight vector controls how each region of the response must be shaped. The weighting vector is defined separately for the direct responses 
and the cross-talk responses. The weight vector $w_{\mathrm{DR}}$ for the direct responses $[27,28]$ can be defined as

$$
w_{\mathrm{DR}}(n)= \begin{cases}1 & \text { for } 1 \leq n \leq N_{1}+1 \\ 0 & \text { for } N_{1}+2 \leq n \leq N_{1}+N_{2} \\ 10^{\frac{3}{\log \left(N_{0} /\left(N_{1}+N_{2}\right)\right)} \log \left(\frac{n}{N_{1}+N_{2}}\right)+0.5} & \text { for } N_{1}+N_{2}+1 \leq n \leq N_{r}\end{cases}
$$

where the region with indices $\left[1, N_{1}\right]$ is the pre-echo, the sample with index at $N_{1}+1$ is the direct path, samples with indices $\left[N_{1}+2, N_{1}+N_{2}\right]$ are the early reflections (don't care regions) and samples with indices $\left[N_{1}+N_{2}+1, N_{\mathrm{r}}\right]$ are the late reverberations. The weights for the late reverberations are chosen in accordance with the temporal masking limit $[3,77,78]$ as in [28]. The human audible range is generally from $20 \mathrm{~Hz}$ to $20 \mathrm{kHz}$, with the threshold of sound pressure level decaying exponentially with increase in frequency. Temporal masking occurs when the perception of a sound is affected by another sound in time. There is a masking threshold up to which the human ear cannot understand the effect of masking. This effect is exploited to reduce some of the weaker sounds (late reverberation) and achieve the desired shaping without significantly affecting the perception. The use of a weight vector based on temporal masking limit requires a parameter $N_{0}=0.2 F_{\mathrm{s}}+0.004 F_{\mathrm{s}}[28]$ where $F_{\mathrm{s}}$ is the sampling frequency. The choice of this parameter is based on the fact that the masking curve starts with $-10 \mathrm{~dB}$ at $4 \mathrm{~ms}$ after the end of 'don't care' region and then decays exponentially in the logarithmic domain to $-60 \mathrm{~dB}$ at $200 \mathrm{~ms}$ after the end of the 'don't care' regions [28]. For channels with late reverberations longer than $200 \mathrm{~ms}$, this parameter has to be changed as $N_{0}=t_{0} F_{\mathrm{s}}+0.004 F_{\mathrm{s}}$, where $t_{0}=T_{60}-N_{1} / F_{\mathrm{s}}-N_{2} / F_{\mathrm{s}}$ i.e., the length of the late reverberation up to the reverberation time.

In the case of the cross-talk paths, the weight vector $w_{\mathrm{CT}}$ controls the cross-talk and is defined as

$$
w_{\mathrm{CT}}(n)=1 \text { for } 1 \leq n \leq N_{\mathrm{r}}
$$

which means that there is no desired part in the cross-talk and all of its samples must be minimized. The weight vector $w$ is constructed from $w_{\mathrm{DR}}$ and $w_{\mathrm{CT}}$. For example, for the case of $L=3, M=2, w=\left[\begin{array}{llll}w_{\mathrm{DR}}^{\mathrm{T}} & w_{\mathrm{CT}}^{\mathrm{T}} & w_{\mathrm{CT}}^{\mathrm{T}} & w_{\mathrm{DR}}^{\mathrm{T}}\end{array}\right]$.

The weight vectors are plotted in Figure 4.2 for a channel sampling frequency $F_{\mathrm{s}}$ of $44.1 \mathrm{kHz}$, channel length $N_{\mathrm{c}}=11000$ and GIR length $N_{\mathrm{r}}=32768$. It can be seen that the weights are at one for the pre-echo and cross-talk implying that 


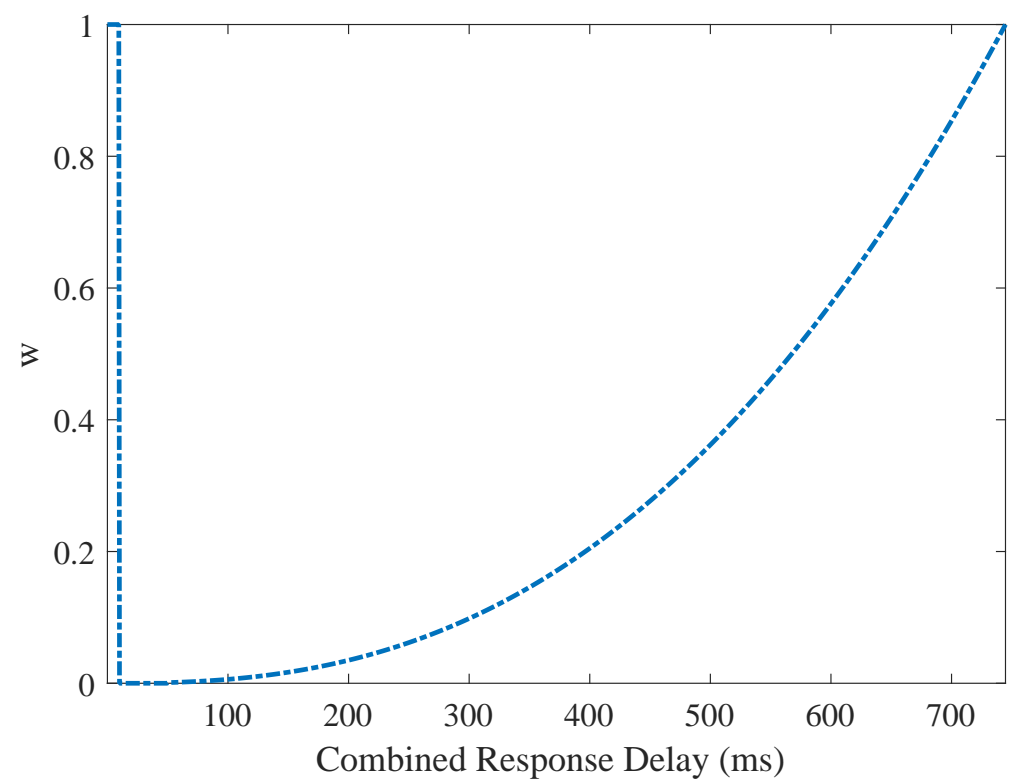

Figure 4.2: Weight vector for $N_{\mathrm{r}}=32768$ and $F_{\mathrm{s}}=44.1 \mathrm{kHz}$.

they are to be minimized as much as possible. The weights are set at zero for the early reflections, which are effectively a 'don't care' region since early reflections are perceptually useful. The weights increase rapidly after the early reflections to minimize the late reverberations. It can be seen that the weights increase with sample number for the late reverberations, implying that the late echoes are minimized.

The desired vector $W r$ is set as zero at all samples except for the direct path $\left(N_{1}+1\right)$. This makes the solution strive towards ensuring that the direct path is always at 1 , while the other regions of the response are shaped. The direct path has been given a weight 1 which ensures that the GIR has a value of 1 at the direct path when the weighted error is minimum. Thus, the shaped direct response appears as shown in Figure 4.3, with a low pre-echo region, a peak corresponding to the direct path, a 'don't care' region and a rapidly decreasing late reverberation region. The early reflection or 'don't care' region and some of the early parts of later reverberation are shown close up in Figure 4.4. In the case of the cross-talk response, the maximum cross-talk level is maintained at the level of pre-echo. 


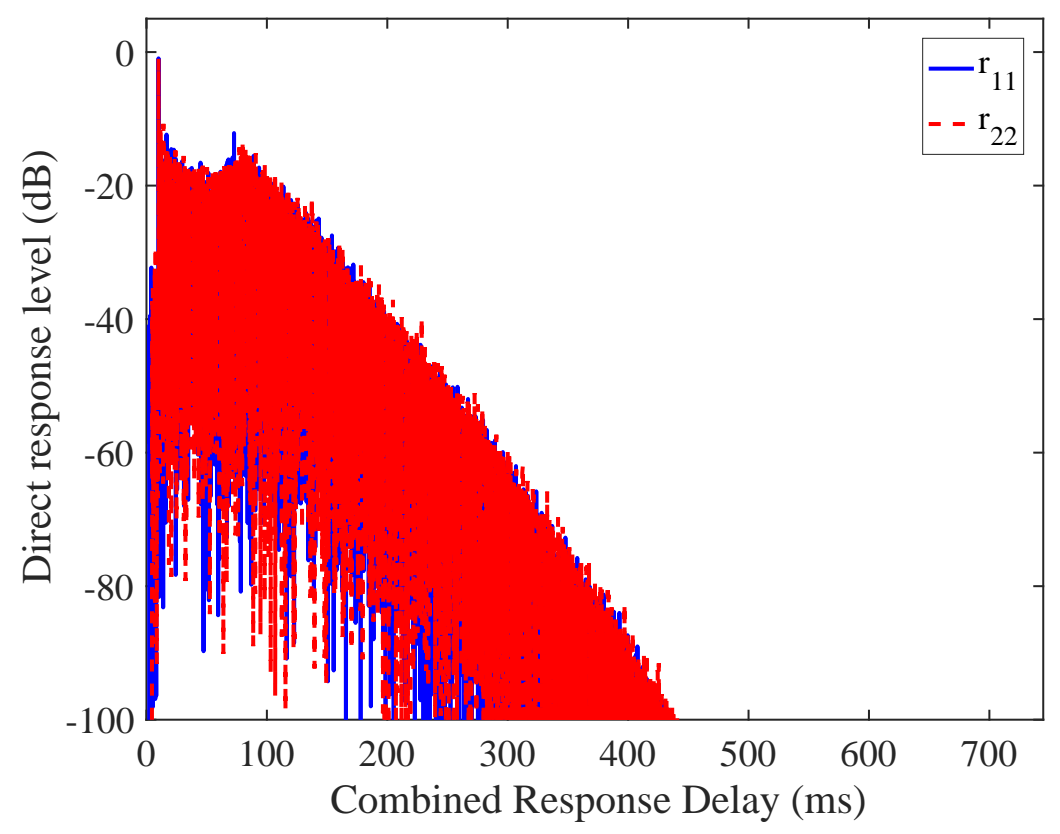

Figure 4.3: Shaped direct responses $r_{11}$ and $r_{22}$.

\subsection{Regularized shaping formulation}

The joint shaping and cross-talk cancelation problem can be formulated as a regularized minimization problem of the form

$$
\min _{h}\|W C h-W r\|_{u}+\lambda\|h\|_{v}
$$

where $u$ is the norm of the fidelity factor and $v$ is the norm of the regularizer. The fidelity factor represents the constraint and the regularizer ensures that the filter coefficients are not very large. Thus, the regularizer plays an important role in ensuring the robustness and stability of the designed filters. $\lambda$ is the regularization parameter that decides the relative importance of these two terms. In this thesis, $u \in\{2, \infty\}$ and $v \in\{1,2\}$ are used. The use of an $\ell_{2}$ norm based regularizer ensures that the energy of the shaping filters are minimized and can be used in applications where low energy filters are desired. When $u=v=2$, the minimization problem is in the form of an $\ell_{2}$ regularized $\ell_{2}$ minimization or the standard Tikhnov regularized [74] LS solution.

The use of an $\ell_{1}$ norm based regularizer in the minimization problem encourages sparsity in the computed filters. Strictly speaking, sparsity is maximized by 


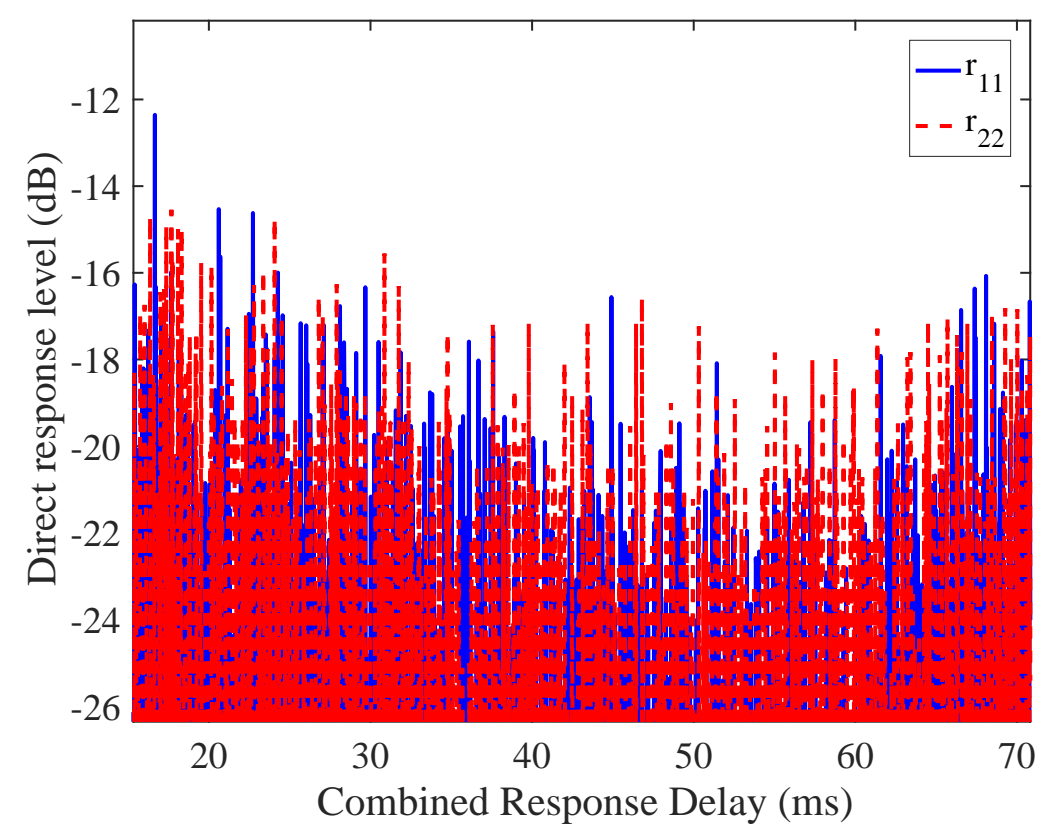

Figure 4.4: Early reflection region of the direct responses.

minimizing the $\ell_{0}$ norm, although with high probability, the $\ell_{1}$ norm minimization gives a similarly sparse solution [37] and obtaining a truly sparseness maximizing solution is not central to this problem. When $u=2$ and $v=1$, the minimization problem is in the form of an $\ell_{1}$ regularized $\ell_{2}$ minimization as in sparse channel estimation [103]. The sparse approach was adopted for this problem for two main reasons. Firstly, including a regularized $\ell_{1}$ norm of $h$ into the optimization problem improves (like the $\ell_{2}$ norm) the robustness of the solution and reduces the energy. Secondly, this allows the use of the high computational efficiency and speed of iterative sparse reconstruction algorithms such as fast DALM [34-36] to estimate a feasible solution for $h$. This is particularly important because for the typical room response sizes of 1000 s to 10000 s of taps, the optimization problem defined in (4.5) becomes computationally challenging and may potentially consume large amounts of computer time to numerically obtain the shaping filters using conventional techniques such as gradient projection and interior point methods.

We initially focus on the $u=2, v=1$ case:

$$
\min _{h} \frac{1}{2}\|W C h-W r\|_{2}^{2}+\lambda\|h\|_{1}
$$


This formulation tries to find a sparse estimate for $h$ that solves the minimization problem posed in (4.5) if the length of $h$ (containing all the filters $h_{l s}$ concatenated) is chosen to be greater than that of $r$ (containing all the GIRs $r_{m s}$ ) so that the matrix $C$ is analogous to an overcomplete dictionary [103]. The sparse estimation problem finds a sparse solution to a linear system of equations with infinite solutions i.e., a sparse estimate for $h$ that satisfies the equation $C h=r$ [103]. Such a formulation is well known in sparse reconstruction as the BPDN problem. This optimization formulation can be solved easily and in a computationally efficient manner by using iterative sparse reconstruction algorithms.

The other formulations considered in this thesis are $\ell_{1}$ regularized $\ell_{\infty}$ minimization and $\ell_{2}$ regularized $\ell_{\infty}$ minimization. These formulations have been developed specifically for the shaping problem since the minimization of the $\ell_{\infty}$ norm of the fidelity factor ensures that the maximum value of the shaping error is minimized, which in turn will result in each element of the GIR vector being likewise constrained. This is of significance because the $\ell_{2}$ minimization may allow some elements to become large and result in late echoes. Either of the $\ell_{1}$ norm or $\ell_{2}$ norm regularizers are beneficial in shaping and can be used depending on the problem requirements. The $\ell_{1}$ norm regularizer can be used if sparse shaping filters are preferred and $\ell_{2}$ norm regularizer if low energy filters are desired.

All these formulations are convex, which can be proved as given below. We prove the convexity for $\ell_{1}$ regularized $\ell_{\infty}$ minimization; others can be proved in a similar fashion.

Let $f(h)=\|W C h-W r\|_{\infty}+\lambda\|h\|_{1}$. For ease of notation, let $A=W C, x=h$ and $b=W r$. Now, $A x-b=A\left(x-A^{\dagger} b\right)$ where $A^{\dagger}$ is the generalized inverse of $A$. Here, $A A^{\dagger}=I$ only if $A$ is wide i.e., length of $h>$ length of $r$. A similar proof can be constructed in the case of a tall $A$ matrix.

Now, $A^{\dagger} b$ is another constant vector $b_{1}$. Let us define a new variable $y=x-b_{1}$. Therefore, $A x-b=A y$.

By definition of convexity, a function $f(y)$ is convex if $\forall y_{1}, y_{2} \in \mathbb{R}, \forall t \in[0,1]$,

$$
f\left(t y_{1}+(1-t) y_{2}\right) \leq t f\left(y_{1}\right)+(1-t) f\left(y_{2}\right)
$$

For the given $f(y), f\left(t y_{1}+(1-t) y_{2}\right)=\left\|t A y_{1}+A(1-t) y_{2}\right\|_{\infty}+\lambda\left\|t y_{1}+(1-t) y_{2}\right\|_{1}$. 
Using the property of a norm that $\|t p\|=t\|p\|$ for scalar $t$ and the triangle inequality $(\|p+q\| \leq\|p\|+\|q\|)$,

$$
\begin{aligned}
& \left\|t A y_{1}+(1-t) A y_{2}\right\|_{\infty}+\lambda\left\|t y_{1}+(1-t) y_{2}\right\|_{1} \\
& \left.\leq t\left\|A y_{1}\right\|_{\infty}+(1-t)\left\|A y_{2}\right\|_{\infty}+\lambda t\left(\left\|y_{1}\right\|_{1}\right)+(1-t)\left\|y_{2}\right\|_{1}\right) \\
& =t f\left(y_{1}\right)+(1-t) f\left(y_{2}\right)
\end{aligned}
$$

The Dual Augmented Lagrangian Method(DALM) [34-36], used in this thesis, is outlined in the following section and detailed in the next chapter.

\subsection{Dual Augmented Lagrangian Method}

The DALM [34-36] approach has been proposed to solve BPDN problems of the form of an $\ell_{1}$ regularized $\ell_{2}$ minimization. A contribution of this thesis is to extend it to other norms as well. For $\ell_{1}$ regularized $\ell_{2}$ minimization, the algorithm computes the dual of the objective function of (4.9) using the Fenchel duality theorem [104]. The detailed derivation is given in Section. 5.2 and Appendix A. The dual problem of (4.9) is given by

$$
\max _{y}(W r)^{T} y \quad \text { subject to }(W C)^{T} y \in B_{1}^{\infty}
$$

where $B_{1}^{\infty}$ is the ball $[34,35]$ defined by

$$
B_{1}^{\infty} \triangleq\left\{x \in \mathbb{R}^{n} \mid\|x\|_{\infty} \leq 1\right\}
$$

and $y$ is the dual variable. The augmented dual Lagrangian and the corresponding iterative equations are presented here. The detailed derivation of this dual from the primal and the iterative steps of the algorithm are explained in chapter 5 . The augmented Lagrangian [34] for the dual is given by

$$
\underset{z \in B_{1}^{\infty}, y}{L(h, y, z)}=-y^{T}(W r)+\lambda \frac{y^{T} y}{2}+\frac{\beta\left\|z-(W C)^{T} y\right\|_{2}^{2}}{2}-h^{T}\left(z-(W C)^{T} y\right)
$$

where $z$ is the dual variable corresponding to projection onto the $B_{1}^{\infty}$ ball, $\beta$ is a regularization parameter and the third term containing an $\ell_{2}$ norm is the augmentation term. The augmentation term is added to enhance the convergence. Addition of this term does not affect the final solution since the term reduces 
to 0 at the solution point. The update equations for $h, y$ and $z$ are found from the derivative of the dual Lagrangian with respect to each of the corresponding variables. The algorithm can finally be written as an iteration of three steps given by

$$
\begin{aligned}
& z_{k+1}=P_{B_{1}^{\infty}}\left((W C)^{T} y_{k}+\frac{h_{k}}{\beta}\right) \\
& y_{k+1}=\left((W C)(W C)^{T}+(\lambda / \beta) I\right)^{-1}\left((W C) z_{k+1}-\frac{(W C) h_{k}-(W r)}{\beta}\right) \\
& h_{k+1}=h_{k}-\beta\left(z_{k+1}-(W C)^{T} y_{k+1}\right)
\end{aligned}
$$

where $P_{B_{1}^{\infty}}$ is the projection onto the $B_{1}^{\infty}$ ball to ensure that $\|z\|_{\infty} \leq 1$, performed by applying $z_{i}=\operatorname{sign}\left(z_{i}\right) \min \left(1,\left|z_{i}\right|\right)$ to each element of the vector $z$.

\section{Fast Dual Augmented Lagrangian Method}

Fast Dual Augmented Lagrangian Method (Fast DALM) [34] is a computationally efficient and faster version of DALM, which involves a few additional optimization steps in order to increase the computational speed. The matrix $\lambda I+\beta(W C)(W C)^{T}$ in the $y$-update of (4.15) can become large and hence the update equation of $y$ can be computationally expensive. The matrix inversion in (4.15) can be replaced by one iteration of the conjugate gradient (CG) algorithm [105] which is advantageous compared to other $\ell_{2}$ norm based algorithms like ISTA, DALM, MINT and RMCLS [22] that use matrix inversions. The fast DALM algorithm can be summarized [34] as

$$
\begin{array}{ll}
\text { Step 1: } & z_{k+1}=P_{B_{1}^{\infty}}\left((W C)^{T} y_{k}+\frac{h_{k}}{\beta}\right) \\
\text { Step 2: } & g_{k}=\lambda y_{k}-b+\beta(W C)(W C)^{T} y_{k}-\beta(W C) z_{k+1}+(W C) h_{k} \\
& \alpha_{k}=g_{k}^{T} g_{k} /\left(\lambda g_{k}^{T} g_{k}+\beta g_{k}^{T}(W C)(W C)^{T} g_{k}\right) \\
& y_{k+1}=y_{k}-\alpha_{k} g_{k}
\end{array}
$$

Step 3: $h_{k+1}=h_{k}-\beta\left(z_{k+1}-(W C)^{T} y_{k+1}\right)$

\subsection{Performance Metrics}

This section explains the performance metrics to evaluate the performance of various shaping formulations developed in this thesis. The metrics are 
- Cross-talk to Direct Response Ratio (CDR)

CDR is the ratio of the maximum value of the cross-talk to the maximum value of the direct response expressed in $\mathrm{dB}$. It is the inverse of DSCR (Direct Signal to Cross-talk Ratio) used in [28]. CDR quantifies the cross-talk cancelation performance of the formulation. It is given by

$$
\mathrm{CDR}=20 \log _{10}\left(\frac{\max \left(r_{\mathrm{ms}}\right)}{\max \left(r_{\mathrm{mm}}\right)}\right) \text { such that } m \neq s, m=1 \ldots M
$$

- CPU time

This metric is used to study the computational speed of the algorithm used to solve for the shaping filters. It quantifies the time taken in seconds by the algorithm to converge to a solution. It is also used to compare the execution speed of various formulations developed in this work.

- Number of iterations

This metric is also used to study the computational speed of the algorithm. The CPU time and number of iterations together give the average time taken for each iteration step of the algorithm.

- Objective function value

This metric quantifies the final objective function value of (4.8) in $\mathrm{dB}$ achieved by the algorithm. It can be used to analyze the convergence of the algorithm.

- Perceived Reverberation Quality measure (nPRQ) [28]

This metric is widely used in the literature to analyze the reverberation performance of the shaped responses. It is expressed as the normalized sum of all sample values of the late reverberation that exceed -60 dB. It is calculated as

$$
\operatorname{nPRQ}(\mathrm{dB})=\frac{\sum_{n=1}^{M N_{\mathrm{r}}} g_{\mathrm{E}}(n)}{\left\|g_{\mathrm{E}}\right\|_{0}}
$$

where

$$
g_{\mathrm{E}}(n)= \begin{cases}20 \log _{10}\left(r_{\mathrm{mm}}(n)\right)-(-60 \mathrm{~dB}) & \text { for } 20 \log _{10}\left(r_{\mathrm{mm}}(n)\right)>(-60 \mathrm{~dB}) \\ 0 & \text { otherwise }\end{cases}
$$

- Spectral Flatness Measure (SFM) [106, 107]

This metric is used to analyze the flatness of the frequency spectrum of a response. SFM values for any response lie in the range $[0,1]$ with $\mathrm{SFM}=1$ 
indicating perfect flatness. It is calculated mathematically as the ratio of geometric mean of the power spectrum to its arithmetic mean. For a signal $x(n)$, the SFM is given by

$$
\mathrm{SFM}=\frac{\sqrt[N]{\prod_{n=0}^{N-1} x(n)}}{\sum_{n=0}^{N-1} x(n) / N}
$$

This metric is used in Chapter 6 to analyze the frequency shaping performance of the algorithm developed.

- Direct to Reverberation Ratio (DRR) [62]

This metric is used in literature on reverberation control to study the relative energy content in the direct path and reverberation components. It is calculated as the ratio of energy in direct path to energy in reverberation. For a GIR $r_{m m}$ with direct path at $N_{1}+1$ sample and late reverberation from sample $N_{1}+N_{2}+1$ to $N_{\mathrm{r}}$, DRR is calculated as

$$
\operatorname{DRR}(\mathrm{dB})=20 \log \frac{r_{m m}^{2}\left(N_{1}+1\right)}{\sum_{n=N_{1}+N_{2}+1}^{N_{\mathrm{r}}} r_{m m}^{2}(n)}
$$

This metric is used in Chapter 8 to analyze the extend of pre-echo and postreverberation control achieved with the pressure matching formulation.

\subsection{Chapter Summary}

The present chapter has introduced the optimization formulation of the shaping problem, including the design of the weight vector for different regions of the room response. The chapter also discussed the regularized shaping formulation, which helps to control the characteristics of the shaping filters. The various regularized formulations considered in this thesis are outlined along with their advantages. Two regularizers are considered in this thesis, viz.; $\ell_{1}$ norm and $\ell_{2}$ norm. The $\ell_{1}$ norm regularizer can be used if sparse shaping filters are desired. Inclusion of $\ell_{1}$ norm regularizer results in a new regularized impulse response shaping formulation that encourages sparsity. The sparse reconstruction algorithm fast DALM is used for solving for the shaping filters. The $\ell_{2}$ norm regularizer can be used if low energy shaping filters are desired. The fast DALM algorithm has been extended to deal with the $\ell_{2}$ regularized problems. The chapter also introduces the various performance metrics used to analyze the work presented in this thesis. The next 
chapter discusses the DALM and the fast DALM algorithms in detail including some formulations of fast DALM for the shaping problem. 


\section{Chapter 5}

\section{Fast Algorithms for Shaping}

\subsection{Background}

Impulse response shaping designs pre-filters to partly equalize the regions of impulse response, as seen from the previous chapters. A basic drawback of previous acoustic reshaping approaches is the large amount of computation time required to obtain the shaping filters. This is mainly due to the necessity of dealing with long room impulse responses. It takes a large amount of computation time to converge to a solution with conventionally used techniques such as gradient descent. In the case of the most commonly used steepest descent approach, the convergence rates are only linear $[20,21,23,32]$. In addition, the steepest descent approaches in $[20,21,32]$ requires choosing a suitably varying step size parameter or the use of a line search algorithm [41, p. 464-466]. Even then an unacceptable number of iterations may be required for convergence, especially for very large problems which are usually poorly conditioned. Use of interior point methods as in [27] guarantees rapid quadratic convergence rates in a small number of iterations. However, a Hessian matrix must be formed, the dimensions of which are large, being proportional to the number of taps in the RIRs. Thus, the memory requirements are prohibitive with the use of interior point methods for this problem. This highlights the importance of using algorithms which are not only fast converging but also memory efficient to solve for the shaping filters. Moreover, since the shaping formulation is convex, there is no chance of the iteration getting stuck on a local minima. Hence, non-greedy algorithms with faster convergence can be used for solving for shaping filters.

In this work, the shaping problem is formulated as a regularized minimization 
and solved using the fast Dual Augmented Lagrangian Method (Fast DALM) [34]. The DALM $[35,36]$ algorithm is a non-greedy algorithm, which has been originally proposed to provide a sparse solution for the $\ell_{1}$ regularized $\ell_{2}$ minimizations (D2-1) as in the BPDN [37] problems that arise in sparse reconstruction. (For the sake of simplicity in notation, we define the algorithms as $\mathrm{D}-n-r$ where $n$ is the norm of the fidelity factor and $r$ is the norm of the regularizer.) This algorithm is known for its super-linear convergence [35] that enables the shaping filters to be found in a computationally fast fashion. Fast DALM is a fast version of DALM that further reduces the computational time. In addition, the iterative steps of the fast DALM algorithm can be easily modified using a Forward Adjoint Oracle (FAO) [108] approach to avoid the use of huge matrices and as a result can be memory efficient.

This chapter formulates the shaping problem in the form of D-2-1, $\ell_{2}$-regularized $\ell_{2}$-norm minimization (D-2-2), $\ell_{1}$-regularized $\ell_{\infty}$-norm minimization (D- $\infty$ - 1 ) and $\ell_{2}$-regularized $\ell_{\infty}$-norm minimization (D- $\infty$-2) and derives the corresponding steps of the DALM algorithm to numerically find the solution. The D-2-2 formulation is the standard Tikhnov regularized minimization, whose solution can be easily computed analytically. The DALM based method provides a faster solution process that avoids the matrix inverse that appears in the analytical solution. The D- $\infty-1$ and D- $\infty-2$ problems are considered specifically for the shaping problem. The minimisation of the $\ell_{\infty}$ norm of the fidelity factor ensures that the maximum value of the shaping error is minimized, which in turn will result in each element of the GIR vector being likewise constrained. This is of significance because D-2-2 can allow some elements to become large and result in late echoes. In the case of D-2-1 and D- $\infty-1$, the regularizer is the $\ell_{1}$ norm which results in sparse shaping filters. When an $\ell_{2}$ norm regularizer is used as in D-2-2 and D- $\infty-2$, the energy of the shaping filters is minimized. Either of these regularizers are beneficial in shaping and can be used depending on the problem requirements.

The contents of this chapter are based on the papers [109-111].

\subsection{Dual Augmented Lagrangian Method}

The Dual Augmented Lagrangian Method (DALM) [34-36] approach was originally designed to solve BPDN problems [37] of the form $A x+n=b$ where $n$ is 
Additive White Gaussian Noise (AWGN), such as the problem,

$$
\min _{x} \frac{1}{2}\|A x-b\|_{2}^{2}+\lambda\|x\|_{1}
$$

The derivation of the DALM approach for D-2-1 problems is detailed here. The DALM approach can also be extended to the use of other norms of the fidelity and regularization metrics as explained in subsequent sections.

The DALM optimization method as presented in [35] aims at solving minimization problems of the form

$$
\min _{x} f(x)+\lambda\|x\|_{1}
$$

that include an $\ell_{1}$ norm term in the objective function. This can be written equivalently as

$$
\min _{x} \frac{1}{\lambda} f(x)+\|x\|_{1}
$$

Using $(1 / \lambda) f(x)=f_{1}(x)$, the problem can be restated as

$$
\min _{x} f_{1}(x)+\|x\|_{1}
$$

The first step in finding the dual problem is to find the Fenchel conjugates of the fidelity term and the regularizer. The conjugates are then combined according to the Fenchel duality theorem to find the dual. This procedure is detailed in Appendix. A. The dual of (5.4) is given by (A.6).

\subsubsection{Special Cases}

\section{$\ell_{2}$ norm}

Let $f_{1}(x)=\|A x-b\|_{2}^{2} / 2 \lambda$. Using the Fenchel conjugate of $\|x-b\|_{2}^{2} / 2$ given by (A.15) into the general form of dual problem given by (A.9) in Appendix. A, the dual problem can be written as

$$
\begin{array}{ll}
\min _{y, z} & -y^{T} b+\frac{\lambda y^{T} y}{2}+I_{1}^{\infty}(z) \\
\text { subject to } & z=A^{T} y
\end{array}
$$

where $I_{1}^{\infty}(z)$ is the indicator function of the $\ell_{\infty}$ norm. The norm indicator function is defined in (A.18). 


\section{$\ell_{\infty}$ norm}

If $f(x)=\|A x-b\|_{\infty}$, the primal objective is of the form

$$
\min _{x}\|A x-b\|_{\infty}+\lambda\|x\|_{1}
$$

Substituting (A.17) into (A.10), the dual is

$$
\begin{array}{ll}
\max _{y, z} & -\left(-y^{T} b\right)-I_{\lambda}^{\infty}(z)-I_{1}^{1}(y) \\
\text { subject to } & z=A^{T} y
\end{array}
$$

where $I_{1}^{1}(y)$ is the indicator function of the $\ell_{1}$ norm.

\subsubsection{Algorithm for D-2-1 objective function}

The Dual Augmented Lagrangian uses an augmented $\ell_{2}$ term in the dual in order to obtain super linear convergence [35]. The dual augmented Lagrangian for a dual problem of the form (5.5) as proposed in [34] is of the form

$$
\underset{z \in B_{1}^{\infty}, y}{L(x, y, z)}=-y^{T} b+\lambda \frac{y^{T} y}{2}+\frac{\beta\left\|z-A^{T} y\right\|_{2}^{2}}{2}-x^{T}\left(z-A^{T} y\right)
$$

where the third term contains an $\ell_{2}$ norm augmentation term and the fourth term is the Lagrange multiplier term corresponding to the constraint $z=A^{T} y$. It can be seen that the augmentation term is convex. The addition of the augmentation term and minimization of the new objective does not change the solution (since the augmentation term is zero when $z=A^{\mathrm{T}} y$ ), but results in its faster convergence [35]. The value of $\beta$ is chosen between 0 and 1 [34]. At the solution to (5.1), the Karush-Kuhn-Tucher(KKT) conditions include the conditions that the derivative of (5.8) with respect to each of $y$ and $z$ are zero. Equating the derivatives to zero gives the update equations for these variables.

Differentiating $L(x, y, z)$ in (5.8) with respect to $y$ gives

$$
\frac{\partial L}{\partial y}=\lambda y-b+A x+\beta\left(-A z+A^{T} A y\right)
$$

and equating this derivative to zero gives the update equation for $y$ in the DALM algorithm which is of the form

$$
y \leftarrow\left(\beta A A^{T}+\lambda I\right)^{-1}(\beta A z-A x+b)
$$


Similarly, differentiating (5.8) with respect to $z$ gives

$$
\frac{\partial L}{\partial z}=-\beta A^{T} y-x
$$

and since $z \in B_{1}^{\infty}$, the update equation for $z$ is of the form

$$
z \leftarrow P_{B_{1}^{\infty}}\left(A^{T} y+\frac{x}{\beta}\right)
$$

where $P_{B_{1}^{\infty}}$ is the projection onto the $B_{1}^{\infty}$ ball to ensure that $\|z\|_{\infty} \leq 1$, performed by applying $z_{i}=\operatorname{sign}\left(z_{i}\right) \min \left(1,\left|z_{i}\right|\right)$ to each element of the vector $z$. The update equation for $x$ is computed using gradient descent based on $\partial L / \partial x=z-A^{T} y$ of the form

$$
x \leftarrow x-\beta\left(z-A^{T} y\right)
$$

At the optimal point, the constraint $z-A^{T} y$ is satisfied, resulting in $x_{k+1}=$ $x_{k}$, which validates that convergence has occurred. It has been pointed earlier that steepest descent result in slow convergence if the step size is not chosen properly. In the $x$-update, the step size parameter is chosen as $\beta$, which is the augmentation parameter introduced into the dual augmented objective to improve the convergence. $\beta$ is chosen, in general, based on the available data as $\beta=\|b\|_{1} / N$ [34] for a sample data vector $b$ of length $N$ to speed up the convergence. For the shaping problem proposed in this thesis, $\beta$ is chosen as $\beta=\|b\|_{1} /\left(L M N_{\mathrm{r}}\right)$ where $L, M$ and $N_{\mathrm{r}}$ are defined as in Section. 4.1. The three update equations are run successively until convergence. It was observed from our experiments that the ordering of the update steps does not influence the performance of the algorithm.

\subsubsection{Fast Dual Augmented Lagrangian Method}

The matrix $\lambda I+\beta A A^{T}$ can be large and (5.10) can be computationally expensive to calculate. The inversion in (5.10) can be replaced by the conjugate gradient (CG) algorithm [105]. It is usually appropriate to perform only one iteration of CG before performing $x$ and $z$ updates. This step can be summarized as follows: The linear equation in $y$ to be solved using $\mathrm{CG}$ is given by

$$
\left(\beta A A^{\mathrm{T}}+\lambda I\right) y=\beta A z-A x+b
$$

The residual $r_{k}$ can be found as

$$
r_{k}=\beta A z-A x+b-\left(\beta A A^{\mathrm{T}}+\lambda I\right) y=-g_{k}
$$


where $g_{k}$ is the partial derivative $\frac{\partial L}{\partial y}$. Now, according to CG, $p_{k}=r_{k}=-g_{k}$ and

$$
\alpha_{k}=\frac{g_{k}^{\mathrm{T}} g_{k}}{g_{k}^{\mathrm{T}}\left(\beta A A^{\mathrm{T}}+\lambda I\right) g_{k}}
$$

The update equation for $y$ can then be written as

$$
y_{k}+1=y_{k}+\alpha_{k} p_{k}=y_{k}-\alpha_{k} g_{k}
$$

This change in $y$-update results in an algorithm called Fast DALM introduced in [34]. For a dual augmented Lagrangian of the form of (5.8), the final algorithm can be summarised as

$$
\begin{array}{ll}
\text { Step 1: } & z_{k+1}=P_{B_{1}^{\infty}}\left(A^{T} y_{k}+\frac{x_{k}}{\beta}\right) \\
\text { Step 2: } & g_{k}=\lambda y_{k}-b+\beta A\left(A^{T} y_{k}-z_{k+1}+\frac{x_{k}}{\beta}\right) \\
& \alpha_{k}=g_{k}^{T} g_{k} /\left(\lambda g_{k}^{T} g_{k}+\beta g_{k}^{T} A A^{T} g_{k}\right) \\
& y_{k+1}=y_{k}-\alpha_{k} g_{k} \\
\text { Step 3: } & x_{k+1}=x_{k}-\beta\left(z_{k+1}-A^{T} y_{k+1}\right)
\end{array}
$$

Step 2 is equivalent to one iteration of the CG algorithm.

\subsection{Fast algorithm for $\mathrm{D}-\infty-1$ primal objective}

The update equations for $x, y$ and $z$ for the proposed fast DALM implementation of an $\ell_{1}$ regularised $\ell_{\infty}$ primal objective are derived in this section.

The dual augmented Lagrangian for a dual problem of the form (5.7) can be written as

$$
\underset{z \in B_{1}^{\infty}, y}{L(x, y)}=-y^{T} b+\frac{\beta\left\|z-A^{T} y\right\|_{2}^{2}}{2}-x^{T}\left(z-A^{T} y\right)+I_{1}^{1}(y)
$$

The update equations for $x, y$ and $z$ are found from the derivative of the dual Lagrangian with respect to each of the corresponding variables. The update equations for $x$ and $z$ are the same as for the $\ell_{2}$ objective $-(5.13)$ and (5.12). 


\section{Update equation for $y$}

The update for $y$ is found by taking the partial derivative with respect to $y$ of $L(x, y, z)$ in $(5.19)$.

$$
g=\frac{\partial L}{\partial y}=-b+A x+\beta\left(-A z+A^{T} A y\right)
$$

The update of $y$ is performed as a two step iteration. The first step is a CG iteration as in Section 5.2.3 which makes use of a step size $\alpha_{k}=g_{k}^{T} g_{k} /\left(\beta g_{k}^{T} A A^{T} g_{k}\right)$ and residual $-g_{k}$. The second step is a projection onto an $\ell_{1}$ norm ball [112] using a soft thresholding function[113]. The soft thresholding function can be written for $n=1 \ldots N$ as

$$
y_{k+1}(n)=\operatorname{soft}\left(y_{k}(n), \lambda_{1}\right)= \begin{cases}0 & \text { if }\left|y_{k}(n)\right|<\lambda_{1} \\ y_{k}(n)-\lambda_{1} & \text { if } y_{k}(n)>\lambda_{1} \\ y_{k}(n)+\lambda_{1} & \text { if } y_{k}(n)<-\lambda_{1}\end{cases}
$$

where $y_{k}(n)$ is the $n$th element of $y_{k} . \lambda_{1}$ is defined as

$$
\lambda_{1}=\frac{\left|\left\|y_{k}\right\|_{1}-1\right|}{N}
$$

which measures the deviation of the $\ell_{1}$ norm from 1 in each iteration.

Thus, the two step update of $y$ can be written as

$$
y_{k+1}=\operatorname{soft}\left(y_{k}-\alpha_{k} g_{k}, \lambda_{1}\right)
$$

which uses a combination of two steps: a conjugate gradient descent for the update of $y$ in the form $y_{k+1}=y_{k}-\alpha_{k} g_{k}$ followed by the application of a soft thresholding function for ensuring that $\left\|y_{k+1}\right\|_{1} \leq 1$.

So for the $\ell_{\infty}$ objective, Step 2 of the algorithm is replaced by

$$
\begin{gathered}
\text { Step 2: } g_{k}=-b+\beta A A^{T} y_{k}-\beta A z_{k+1}+A x_{k} \\
\alpha_{k}=g_{k}^{T} g_{k} /\left(\beta g_{k}^{T} A A^{T} g_{k}\right) \\
\lambda_{1}=\left(\left|\left\|y_{k}\right\|_{1}-1\right|\right) / N \\
y_{k+1}=\operatorname{soft}\left(y_{k}-\alpha_{k} g_{k}, \lambda_{1} / 2\right)
\end{gathered}
$$




\subsection{Fast algorithms for $\ell_{2}$ regularised objective functions}

The proposed fast DALM algorithms for D-2-2 and D- $\infty-2$ objective functions are derived in this section. The procedure is similar to that of the $\ell_{1}$ regularized objective functions. The first step is to find the Fenchel conjugates of regularizer and fidelity terms.

\subsubsection{D-2-2 algorithm}

The objective function to be solved for the $\ell_{2}$ regularized $\ell_{2}$ norm minimization is given by

$$
\min _{x} \frac{1}{2}\|A x-b\|_{2}^{2}+\frac{\lambda}{2}\|x\|_{2}^{2}
$$

For $g(x)=\frac{1}{2}\|x\|_{2}^{2}$, the Fenchel conjugate is

$$
g_{1}^{*}(y)=\sup _{x} y^{T} x-\frac{1}{2}\|x\|_{2}^{2}
$$

The supremum value occurs at $x$ satisfying

$$
\frac{\partial\left(y^{T} x-\frac{1}{2} x^{T} x\right)}{\partial x}=0
$$

This yields $y=x$, which on substitution into (5.26) gives

$$
g_{1}^{*}(y)=\frac{y^{T} y}{2}
$$

Since the primal problem involves a linear transformation of the argument of $f(x)$, the conjugate can be rewritten using (A.3) given by

$$
\min _{x} f(x)-g(A x)=\max _{y} g_{*}(y)-f^{*}\left(A^{T} y\right)
$$

as

$$
\begin{array}{r}
g_{1}^{*}=\frac{z^{T} z}{2} \\
\text { subject to } z=A^{T} y
\end{array}
$$

Then using the conjugate of $f_{1}(x)=\|x-b\|_{2}^{2} / 2 \lambda$ from

$$
f_{1}^{*}(y)=y^{T}(\lambda y+b)-\frac{\lambda^{2} y^{T} y}{2 \lambda}=y^{T} b+\frac{\lambda y^{T} y}{2}
$$


detailed in (A.15), the dual of (5.25) can be written as

$$
\begin{array}{ll}
\min _{y, z} & -y^{T} b+\frac{\lambda y^{T} y}{2}+\frac{z^{T} z}{2} \\
\text { subject to } & z=A^{T} y
\end{array}
$$

The dual augmented Lagrangian can then be written as

$$
\begin{aligned}
L(x, y, z)= & -y^{T} b+\lambda \frac{y^{T} y}{2}+\frac{z^{T} z}{2}+\frac{\beta\left\|z-A^{T} y\right\|_{2}^{2}}{2} \\
& -x^{T}\left(z-A^{T} y\right)
\end{aligned}
$$

Differentiating this with respect to $z$ and equating to 0 gives

$$
\frac{\partial L}{\partial z}=-\beta A^{T} y-x+z(1+\beta)=0
$$

which gives the update equation for $z$ :

$$
z \leftarrow \frac{\left(\beta A^{T} y+x\right)}{(1+\beta)}
$$

which replaces Step 1 of the Fast DALM algorithm for D-2-1 objective function.

\subsubsection{D- $\infty-2$ algorithm}

When the primal problem is in the form of a $\mathrm{D}-\infty-2$ problem,

$$
\min _{x}\|A x-b\|_{\infty}+\frac{\lambda}{2}\|x\|_{2}^{2}
$$

using the Fenchel conjugate of $g(x)=(\lambda / 2)\|x\|_{2}^{2}$ given by

$$
\begin{aligned}
g_{1}^{*}(y) & =\sup _{x} y^{T} x-\frac{\lambda}{2}\|x\|_{2}^{2} \\
& =\frac{y^{T} y}{2 \lambda}
\end{aligned}
$$

along with (A.3), we have

$$
\begin{array}{r}
g_{1}^{*}=\frac{z^{T} z}{2 \lambda} \\
\text { subject to } z=A^{T} y
\end{array}
$$


So using (A.17), the dual can be written as

$$
\begin{array}{ll}
\min _{y, z} & -y^{T} b+\frac{z^{T} z}{2 \lambda}+I_{1}^{1}(y) \\
\text { subject to } & z=A^{T} y
\end{array}
$$

The dual augmented Lagrangian then becomes

$$
\begin{aligned}
L(x, y, z)= & -y^{T} b+\frac{z^{T} z}{2 \lambda}+\frac{\beta\left\|z-A^{T} y\right\|_{2}^{2}}{2} \\
& -x^{T}\left(z-A^{T} y\right)+I_{1}^{1}(y)
\end{aligned}
$$

Differentiating this with respect to $x, y, z$ gives the $z$-update step as (5.33) and $y$-update as (5.24).

\subsection{Forward Adjoint Oracle Approach}

As seen in sections 5.2.3 and 5.4, the DALM algorithm solves the optimization problem through iteration of several steps. The largest memory and computational requirement involved is the creation and storage of matrix $A$ and the matrix multiplication $A^{\mathrm{T}} A$. This can be avoided by using a Fourier implementation to further improve the computational performance. The Fourier implementation is possible because $A$ is a Toeplitz matrix representing convolution in the shaping formulation. In optimisation parlance, this technique can be considered a Forward Adjoint Oracle (FAO) approach [108].

\subsubsection{Implementation of $C h$ using Fast Fourier Transforms}

The channel matrix $C_{m l}$ from (4.3) is a Toeplitz convolution matrix and can be represented as

$$
C_{m l}=F^{-1} D_{m l} F
$$

where $F$ is the $N_{\mathrm{r}}$ point DFT matrix and the diagonal matrix $D_{m l}$ contains the Fourier transform of $\check{c}_{m l}$. ( $\check{c}_{m l}$ is $c_{m l}$ after it has been zero padded to length $N_{\mathrm{r}}$ ). Therefore, the convolution equation $C_{m l} h_{l s}$ can be written as $F^{-1} D F \check{h}_{l s}$ where $\breve{h}_{l s}$ is $h_{l s}$ zero padded to $N_{\mathrm{r}}$. This is equivalent to performing the operation $\mathcal{F}^{-1}\left\{\mathcal{F}\left\{\check{c}_{m l}\right\} \odot \mathcal{F}\left\{\check{h}_{l s}\right\}\right\}$ where $\odot$ represents Hadamard product. Therefore, the 
operation (4.4) can be written for the special case $L=3, M=2$ as

$$
\left[\begin{array}{l}
r_{11} \\
r_{21} \\
r_{12} \\
r_{22}
\end{array}\right]=\left[\begin{array}{l}
\sum_{l=1}^{3} F^{-1} D_{1 l} F h_{l 1} \\
\sum_{l=1}^{3} F^{-1} D_{2 l} F h_{l 1} \\
\sum_{l=1}^{3} F^{-1} D_{1 l} F h_{l 2} \\
\sum_{l=1}^{3} F^{-1} D_{2 l} F h_{l 2}
\end{array}\right]
$$

In general, this can be written as

$$
r_{m s}=\mathcal{F}^{-1}\left\{\sum_{l}\left(\mathcal{F}\left\{\check{c}_{m l}\right\} \odot \mathcal{F}\left\{\check{h}_{l s}\right\}\right)\right\}
$$

which consists of only forward and inverse fast Fourier transform operations, vector multiplications and summation along $L$ loudspeakers. Thus, the complexity $\mathcal{O}\left(N_{\mathrm{r}}^{2}\right)$ of one matrix multiplication has been reduced to $\mathcal{O}\left(N_{\mathrm{r}} \log \left(N_{\mathrm{r}}\right)\right)$ using this implementation.

\subsubsection{Implementation of $C^{\mathbf{T}} y$ using Fast Fourier Transforms}

Using the same principle as in the previous section, $C_{m l}^{\mathrm{T}} y_{m s}$ can be represented as $C_{m l}^{\mathrm{T}} y_{m s}=\left(F^{-1} D_{m l} F\right)^{\mathrm{T}} y_{m s}=F^{\mathrm{T}} D_{m l}\left(F^{\mathrm{T}}\right)^{-1} y_{m s}$. This is equivalent to performing $\mathcal{F}\left\{\mathcal{F}\left\{\check{c}_{m l}\right\} \odot \mathcal{F}^{-1}\left\{y_{m s}\right\}\right\}$. Therefore, $C^{\mathrm{T}} y$ can be written (again for the case $L=$ $3, M=2)$ as

$$
\left[\begin{array}{l}
h 1_{11} \\
h 1_{21} \\
h 1_{31} \\
h 1_{12} \\
h 1_{22} \\
h 1_{32}
\end{array}\right]=\left[\begin{array}{l}
\sum_{m=1}^{2} F D_{m 1} F^{-1} y_{m 1} \\
\sum_{m=1}^{2} F D_{m 2} F^{-1} y_{m 1} \\
\sum_{m=1}^{2} F D_{m 3} F^{-1} y_{m 1} \\
\sum_{m=1}^{2} F D_{m 1} F^{-1} y_{m 2} \\
\sum_{m=1}^{2} F D_{m 2} F^{-1} y_{m 2} \\
\sum_{m=1}^{2} F D_{m 3} F^{-1} y_{m 2}
\end{array}\right]
$$


In general, this can be written as

$$
\check{h}_{l s}=\mathcal{F}\left\{\sum_{m}\left(\mathcal{F}\left\{\check{c}_{m l}\right\} \odot \mathcal{F}^{-1}\left\{y_{m s}\right\}\right)\right\}
$$

which results in a vector $\check{h}_{l s}$ of length $N_{\mathrm{r}}$. The desired result is obtained by truncating $\check{h}_{l s}$ to length $N_{\mathrm{h}}$. This truncation does not cause any error in $\check{h}_{l s}$ provided $c_{m l}$ has been zero padded to length $N_{\mathrm{r}}$ and the terms removed are the results of terms of $y$ multiplied by the added zeroes. This is equivalent to deleting $N_{\text {h }}$ rows from the matrix $F$. Here again, the complexity $\mathcal{O}\left(N_{\mathrm{r}}^{2}\right)$ has been reduced to $\mathcal{O}\left(N_{\mathrm{r}} \log \left(N_{\mathrm{r}}\right)\right)$ using this implementation.

\subsection{Design over multiple microphone positions}

The DALM algorithms derived in the previous sections were modified to find shaping filters robust to change in microphone positions. Radlovic et al. [38] shows that the channels are perturbed by changes in microphone position. They describe a theoretical framework to analyze robustness of sound equalization to the change of microphone and source positions in a room and derive a model for the mean square error in estimation due to such a change in position. The mean square error at a frequency $f$ is given by $(2.14)$

$$
\varepsilon(f)=E\left\{|\tilde{C}(f) H(f)-1|^{2}\right\}
$$

where $\tilde{C}(f)$ is the frequency response between the source and the receiver at a distance from the equalization point and $H(f)$ is the frequency response of the inverse filter. Let $d$ be the displacement from the equalization point and $k$ the wave number. If displacement from the equalization point is small compared to the source to microphone distance, the error in the case of only diffuse field is of the form in (2.17) given by

$$
\varepsilon(f) \cong 2-2 \frac{\sin (k d)}{k d}
$$

Based on this idea, a robust design method is proposed in [39] which designs the filters over multiple perturbed positions so that the shaping filters are effective at all these positions. This is obtained by generating perturbations that follow the characteristics described in [38]. First, white noise is generated and its frequency response is multiplied with $\varepsilon(f)$ in (2.14). This is converted back into the time 
domain and multiplied with a time domain shaping function that follows the characteristics of the room response. This gives the perturbation which is added to the room response to get the perturbed response. This process is repeated for all the channels and all the perturbed positions and the shaping filters are designed to be robust to all of the perturbed channels. In this thesis, the DALM algorithms have been modified in order to find such a set of robust shaping filters. In the case of D-2-1 and D-2-2, this can be achieved by modifying the objective function as

$$
\min _{h} \frac{1}{2} \sum_{n=1}^{N_{\mathrm{p}}+1}\left\|W C^{n} h-W r\right\|_{2}^{2}+\frac{1}{p} \lambda\|h\|_{p}
$$

where $N_{\mathrm{p}}$ is the number of perturbations, $C^{n}$ is a perturbed channel, $p=1$ for D-2-1 and $p=2$ for D-2-2. The summation is performed over the original channel $C$ and $N$ perturbed channels, i.e., a total of $N_{\mathrm{p}}+1$ channels. The algorithms are modified accordingly. In the case of $\mathrm{D}-\infty-1$ and $\mathrm{D}-\infty-2$, the robust solution is found by solving

$$
\min _{h}\|W \breve{C} h-W r\|_{\infty}+\frac{1}{p} \lambda\|h\|_{p}
$$

where

$$
\breve{C}=\left[\left(C^{1}\right)^{\mathrm{T}} \ldots\left(C^{n}\right)^{\mathrm{T}} \ldots\left(C^{N_{\mathrm{p}}+1}\right)^{\mathrm{T}}\right]^{\mathrm{T}}
$$

where $N_{\mathrm{p}}$ is the number of perturbations, $C^{n}$ is a perturbed channel, $p=1$ for $\mathrm{D}-\infty-1$ and $p=2$ for D- $\infty-2$. The fidelity factor is chosen to be $\|W \breve{C} h-W r\|_{\infty}$ in $(5.45)$.

\subsection{Simulation Results}

Experimental simulation studies were performed using measured channels from a room of dimension $3.5 \times 2.5 \times 4.5 \mathrm{~m}$ with $L=3, M=2, N_{\mathrm{c}}=10000$ and $N_{\mathrm{r}}=32768$ and a sampling frequency of $44.1 \mathrm{kHz}$. The $T_{60}$ of the room was $245 \mathrm{~ms}$. The separation between two adjacent loudspeakers was $24 \mathrm{~cm}$ and that between the two microphones was $12 \mathrm{~cm}$. The average loudspeaker to microphone distance was $37 \mathrm{~cm}$. A layout of the setup used in the experiments is shown in Figure 5.1. An nPRQ measure (Perceived Reverberation Quality) [21, 28, 80] described in Section 4.4 is used in this work to analyze the efficacy of the algorithm for reverberation reduction. The unreshaped channel had an nPRQ measure of 7.63. The pre-echo, early reflection and late reverberation regions were defined as 


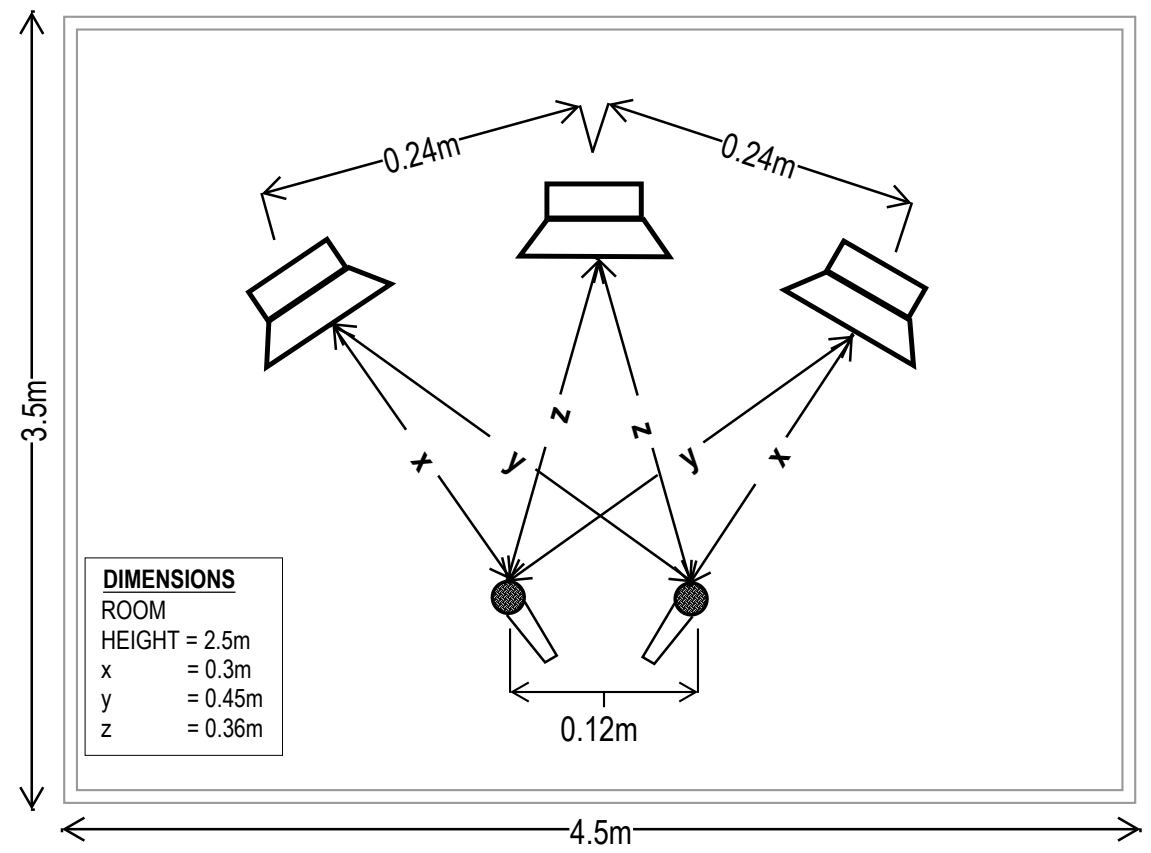

Figure 5.1: Layout of the cross-talk canceller setup used in the simulations and experiments.

$10 \mathrm{~ms}, 40 \mathrm{~ms}$ and $200 \mathrm{~ms}$ respectively. The weighting vector $w$ was set according to (4.6) for the direct responses and (4.7) for the cross-talk paths. A useful performance metric is the Cross-talk to Direct Response ratio (CDR) which is the ratio of maximum cross-talk to maximum direct response (inverse of the DSCR used in [28]) described in Section 4.4.

The first experimental study was conducted to check the efficacy of shaping and cross-talk cancelation using the DALM formulations used in this work, viz. D-2-1, D-2-2, D- $\infty-1$ and D- $\infty-2$. Figure 5.2 shows an example of the cross-talk responses. All the four algorithms provided an average cross-talk cancelation of about $-58 \mathrm{~dB}$. The direct responses shown in Figure 5.3 show that the peak (direct path) is approximately $20 \mathrm{~dB}$ above the smaller values and the response decays rapidly after the allowed early reflections, thus reducing the late reverberation and providing good shaping. Thus, a CDR of around $-58 \mathrm{~dB}$ was achieved using the DALM approach. The average computation time for finding the shaping filters was found to be $58 \mathrm{~s}$ (3600 iterations). This is faster than the 5000 iterations of [21], around 60 times faster than [27] and around 3 times faster than [110]. 


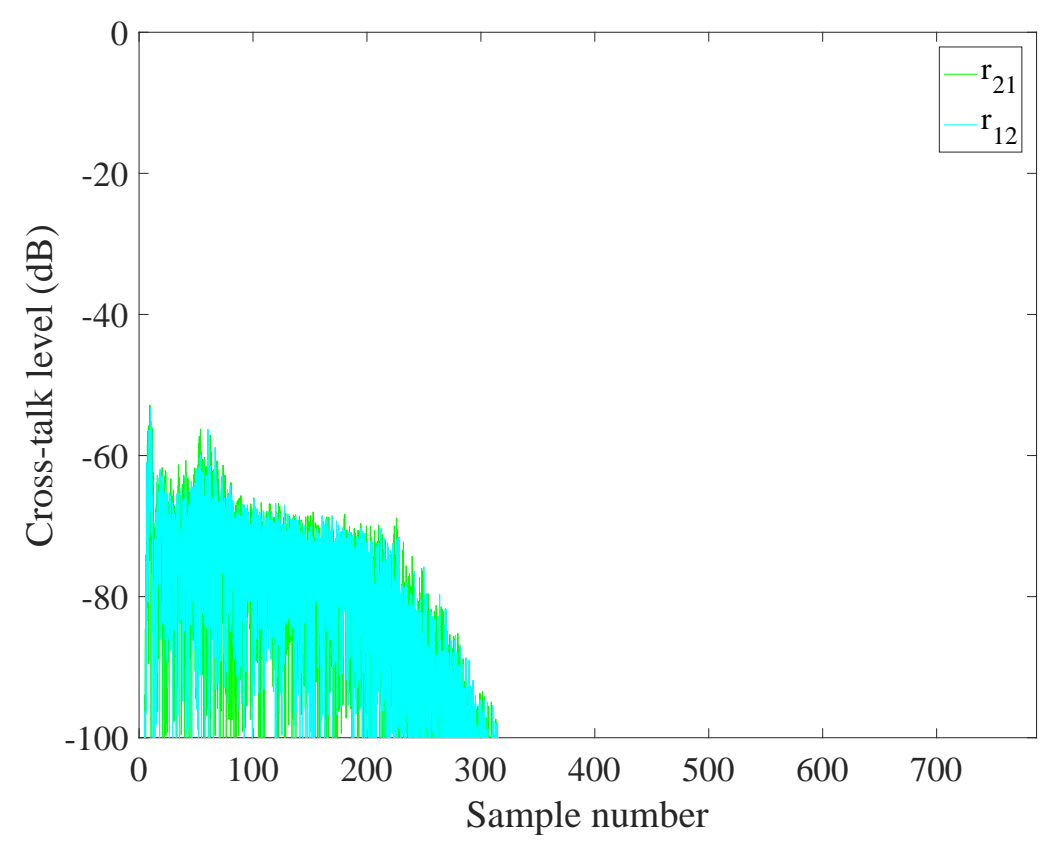

Figure 5.2: Cross-talk responses $r_{12}$ and $r_{21}$.

In order to check the efficacy of the algorithms, the reverberation reduction was quantified using an nPRQ measure, which showed that the DALM approaches provide reverberation reduction comparable to $[28,80]$ along with better crosstalk cancelation. In particular, D-2-1 and D-2-2 were faster since they were solving simpler problems than $\mathrm{D}-\infty-1$ and $\mathrm{D}-\infty-2$. In addition, the $\ell_{2}$ weighted errors $\|W C h-W r\|_{2}^{2}$ were naturally less for D-2-1 and D-2-2 than D- $\infty-1$ and D- $\infty-2$, while the $\ell_{\infty}$ weighted errors $\|W C h-W r\|_{\infty}$ were less for $\mathrm{D}-\infty-1$ and $\mathrm{D}-\infty-2$ than D-2-1 and D-2-2. These results are summarized in Table 5.1.

The algorithms were tested over a range of regularization parameters and lengths of 'don't care' region to study the feasible regions of operation. The crosstalk cancelation performance was quantified by CDR. The variation in CDR for different values of $\lambda$ with $\mathrm{D}-\infty-1$ and $\mathrm{D}-\infty-2$ are shown in Figure 5.4. It can be seen that CDR improves with reduction in $\lambda$. This is in agreement with the observation that as $\lambda$ decreases, more significance is given to minimization of the fidelity factor (which is the weighted error), thus providing better cross-talk reduction.

The variation in CDR for lengths of $N_{2}$ shown in Figure 5.5 indicates that the 


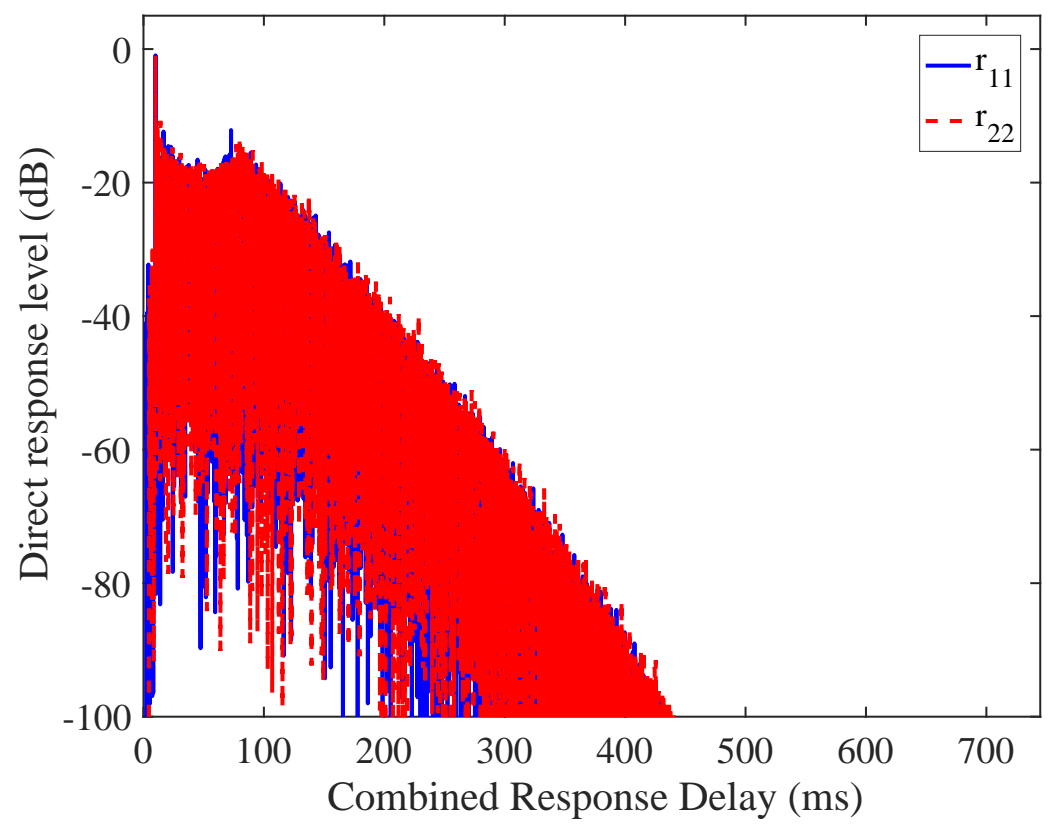

Figure 5.3: Shaped direct responses $r_{11}$ and $r_{22}$.

Table 5.1: Performance of different DALM formulations in shaping. Performance metrics CDR, CPU time and nPRQ are defined as in Section 4.4.

Algorithm $\|h\|_{1} \quad\|h\|_{2} \quad\|W C h-W r\|_{2}^{2} \quad\|W C h-W r\|_{\infty} \quad$ CDR $\quad$ CPU time $\quad$ nPRQ

\begin{tabular}{cccccccc} 
& & & $(\mathrm{dB})$ & $(\mathrm{dB})$ & $(\mathrm{dB})$ & $(\mathrm{s})$ & $(\mathrm{dB})$ \\
\hline $\mathrm{D}-2-1$ & 31.8 & 2.32 & -21 & -57.2 & -57 & 25 & 3.98 \\
\hline $\mathrm{D}-2-2$ & 55.6 & 2.29 & -21.2 & -57.4 & -57 & 28 & 6.26 \\
\hline $\mathrm{D}-\infty-1$ & 41.3 & 2.44 & -20.3 & -58.1 & -58 & 85 & 4.21 \\
\hline $\mathrm{D}-\infty-2$ & 55.1 & 2.31 & -20.42 & -57.96 & -58 & 88 & 4.3 \\
\hline
\end{tabular}

CDR improves with increase in 'don't care' region lengths. As the length of the 'don't care' regions increase, the region of late reverberation to be shaped starts to shrink thus resulting in a comparatively more simple minimisation problem. This in turn results in a pronounced improvement in CDR, though the CDR saturates after the 'don't care region' is 30-40 ms.

The plots of the $\|h\|_{1}$ and $\|h\|_{2}$ values versus $\lambda$ are shown in Figure 5.6 and 


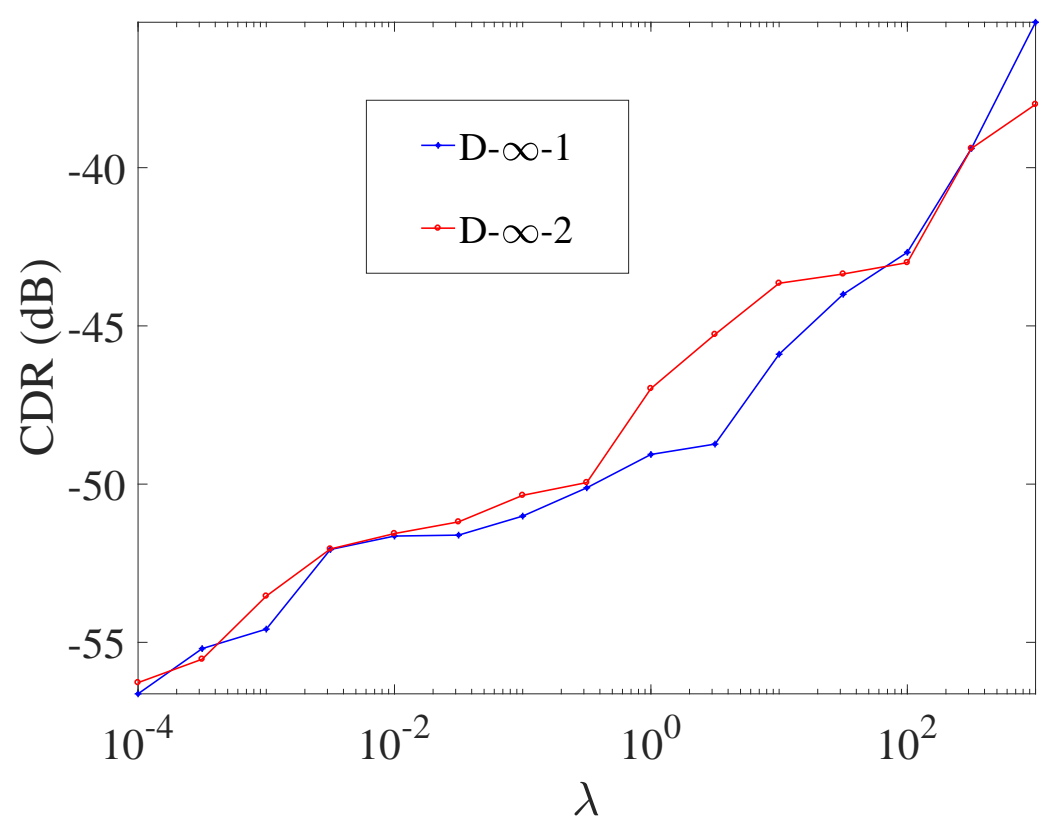

Figure 5.4: Cross-talk cancelation performance with variation in regularization parameter $\lambda$.

Figure 5.7 respectively. It can be seen that as $\lambda$ increases, more significance is given to minimization of the regularizers and $h$ will be robust to variations. But in such a scenario, the weighted error (fidelity factor) value will be higher, resulting in less cross-talk cancelation. Therefore, in order to get a reasonable performance, the $\lambda$ value has to be chosen so that the solution provides good cross-talk cancelation as well as being robust.

The robustness of the DALM algorithms is quantified by showing the Cumulative Density Function (CDF) of the $\ell_{2}$ and $\ell_{\infty}$ errors over an ensemble of different microphone positions. To plot the $\mathrm{CDF}$, the $\ell_{2}$ and $\ell_{\infty}$ errors at the original and 500 position perturbations (at $1 \mathrm{~cm}$ from the original position) were computed. These values are sorted in ascending order and plotted on the horizontal axis of the CDF plot. The vertical axis shows the probability of occurrence $P(X<n)$ of a random variable $X$ lower than the corresponding value $n$ on the horizontal axis. This is shown in Figure 5.8 and Figure 5.9. In addition, the algorithms were modified to design shaping filters over 16 microphone positions (original position and 15 positions at a distance of $1 \mathrm{~cm}$ from it) as described in Section 5.6 and again 


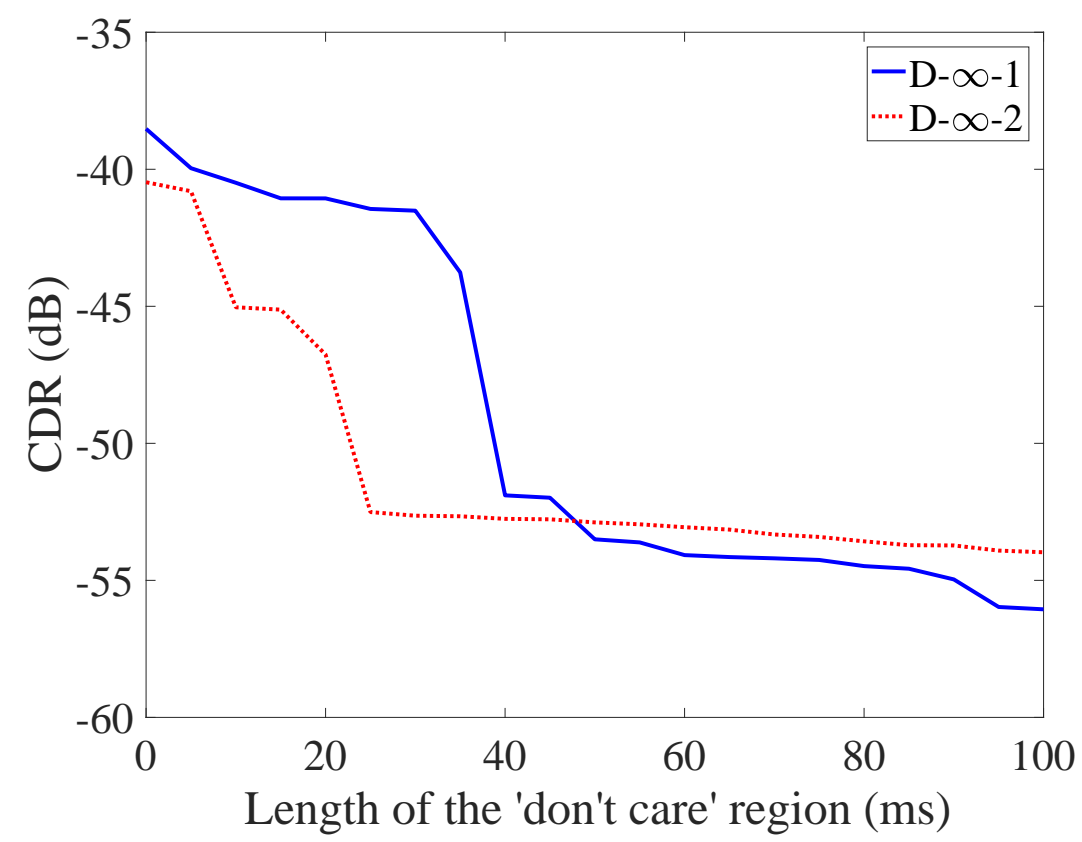

Figure 5.5: Cross-talk cancelation performance with variation in the duration of 'don't care' region.

the errors were computed at the original and 500 perturbed positions. The resultant CDF is shown in Figure 5.10 and Figure 5.11. It was found that the design over multiple positions had a steeper slope than the original DALM approaches (comparing Figure 5.8 with Figure 5.10 and Figure 5.9 with Figure 5.11). This indicates that the variation of errors with a perturbation in the channel is greater for the filter designed without the robust approach. In the non-robust approach, the performance of the filter is good at the designed position, but degraded at the perturbed positions. In the robust approach, the performance of the filters is slightly compromised, but similar at all of the original and perturbed microphone positions. This again highlights the robustness of the proposed approach, when compared with non-robust shaping. These results are summarised in Table 5.2. The nPRQ measures are comparable to $[28,39]$ while the CDR values are better than [28], showing comparable shaping and better cross-talk cancelation. 


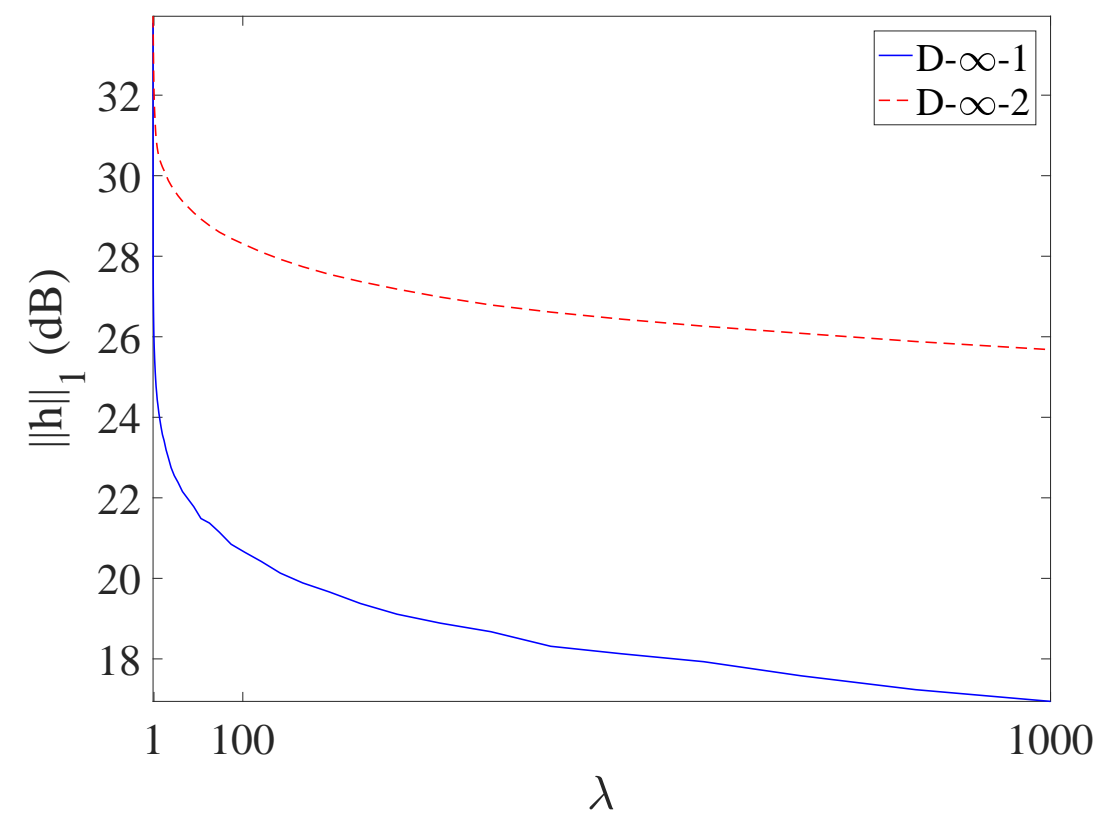

Figure 5.6: Variation of $\ell_{1}$ regularizer function value with $\lambda$.

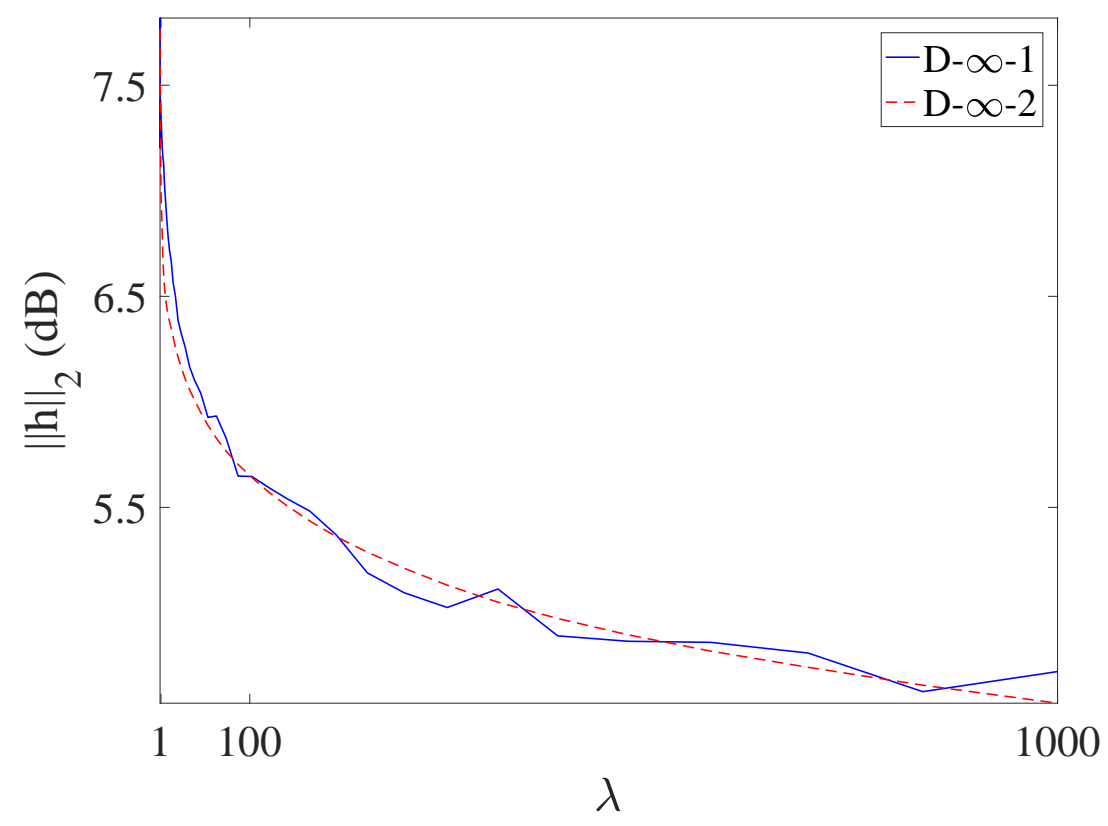

Figure 5.7: Variation of $\ell_{2}$ regularizer function value with $\lambda$. 


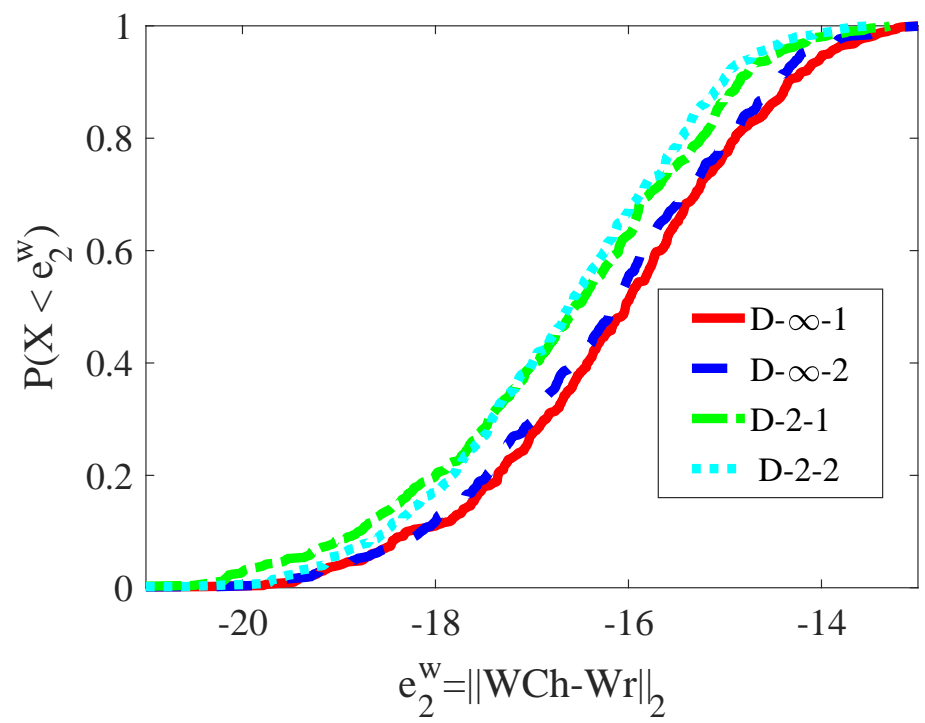

Figure 5.8: Empirical CDF of the $\ell_{2}$ errors at the original and 500 perturbed positions.

Table 5.2: Performance of robust DALM formulations in shaping. Performance metrics CDR, CPU time and nPRQ are defined as in Section 4.4.

Algorithm $\quad\|h\|_{1} \quad\|h\|_{2} \quad\|W C h-W r\|_{2} \quad\|W C h-W r\|_{\infty} \quad$ CDR $\quad$ CPU Time $\quad$ nPRQ

\begin{tabular}{cccccccc} 
& & & $(\mathrm{dB})$ & $(\mathrm{dB})$ & $(\mathrm{dB})$ & $(\mathrm{s})$ & $(\mathrm{dB})$ \\
\hline $\mathrm{D}-2-1$ & 63.4 & 2.99 & -19.2 & -55.5 & -56 & 58 & 3.93 \\
\hline $\mathrm{D}-2-2$ & 121 & 2.72 & -19.18 & -55.1 & -55 & 58 & 5.28 \\
\hline $\mathrm{D}-\infty-1$ & 69.9 & 2.91 & -18.81 & -55.8 & -56 & 914 & 4.09 \\
\hline $\mathrm{D}-\infty-2$ & 79.9 & 2.74 & -18.89 & -55.76 & -56 & 926 & 3.93 \\
\hline
\end{tabular}

\subsection{Experiments in the lab}

Experimental studies were performed in a room of dimension $3.5 \times 2.5 \times 4.5 \mathrm{~m}$ with acoustic buffer pads on all the four walls. The experimental validation of the impulse response formulations were tested using the Validation of Acoustic Channel Shortening (VACS) laboratory setup employed to carry out realtime experiments. The setup was designed and installed in the summer of 2014 using RME audio hardware that consisted of 1 RME MADI FX HDSPe card [114] with 3 RME Micstasy 8 channel pre-amplifiers [115] and a single M32 DAC. The soft- 


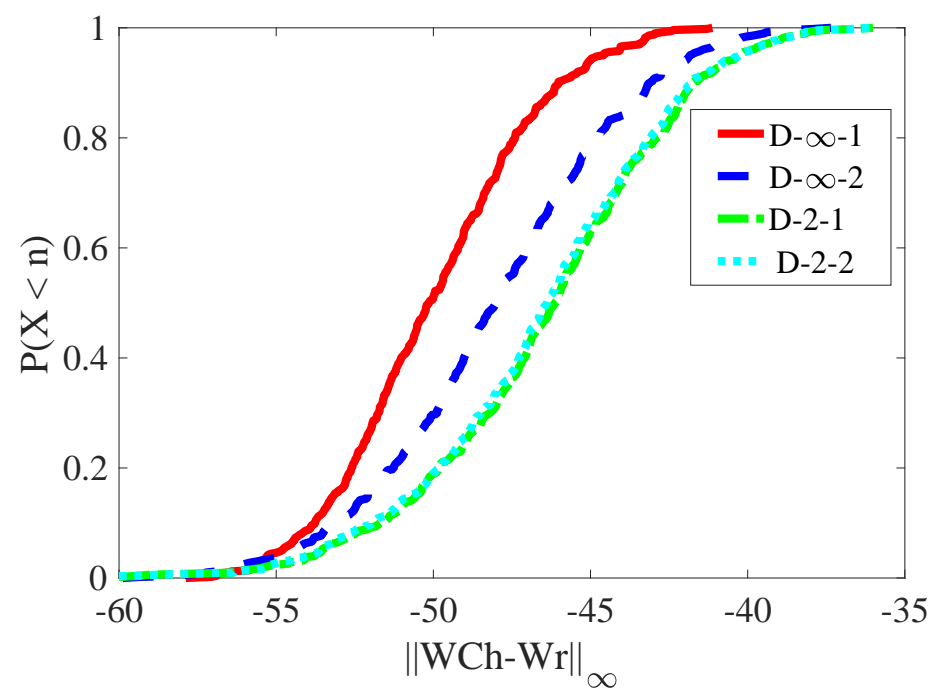

Figure 5.9: Empirical CDF of the $\ell_{\infty}$ errors at the original and 500 perturbed positions.

ware inventory consisted of Matlab script files that uses Digital Signal Processing toolbox and Audio Stream Input/Output (ASIO) drivers installed and selected in the Matlab Digital Signal Processing System Toolbox preferences. The system consists of 24 loudspeakers mounted on a circular shelf in the form of a ring and numbered sequentially from 1 to 24 in anti-clockwise direction. Microphones were mounted on a stand in the form of a ring to approximate the listening area around a human head.

Matlab was used to run the full test from gathering the impulse responses using chirps through each loudspeaker and each microphone to tailoring of shaping filters and measurement of the shaped channels. The impulse response gathering function of the experimental setup allows for any specified matrix of loudspeakermicrophone arrangement to be used. For the current setup, an $L=3, M=2$ arrangement was used to match the simulation conditions. Three adjacent loudspeakers and two adjacent microphones in front of them were chosen. All the three loudspeakers were sequentially sounded using chirp signals and the resulting signals received at the two microphones were measured. The room transfer function is evaluated using

$$
Y(f)=H(f) X(f)
$$

where $X(f)$ and $Y(f)$ are the loudspeaker output signal and the desired micro- 


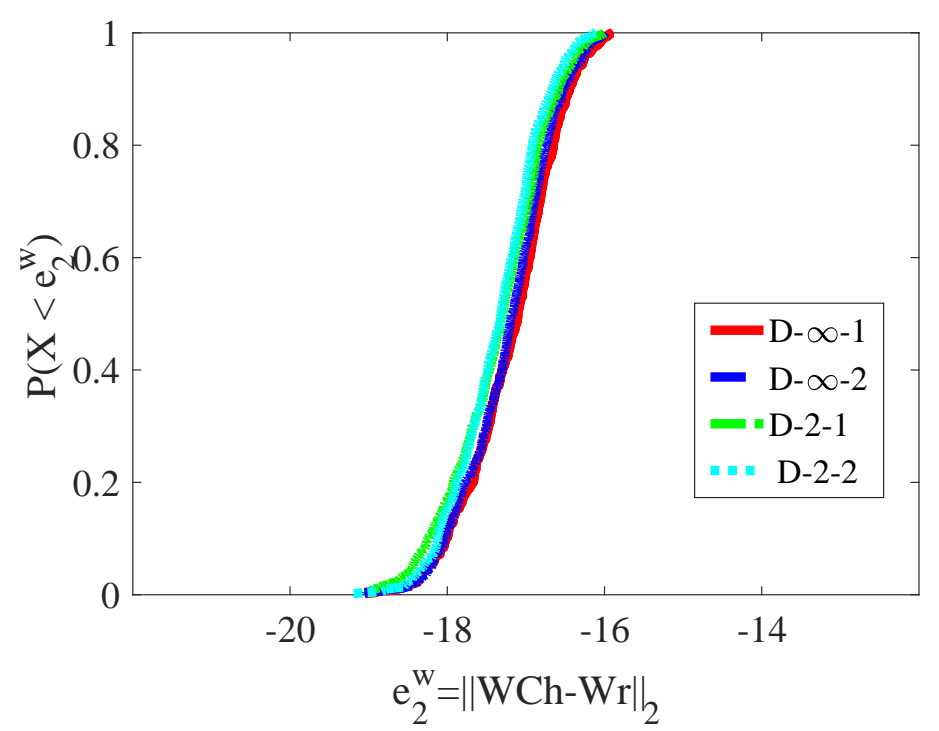

Figure 5.10: Empirical CDF of the $\ell_{2}$ errors at the original position and 500 perturbed positions at $1 \mathrm{~cm}$ from the original position using the robust approach of design over the original position and 15 perturbed positions at $1 \mathrm{~cm}$ from the original position.

phone output respectively. Since $Y(f)$ and $X(f)$ are known, the RTF $H(f)$ can be determined. The inverse Fourier transform of $H(f)$ is windowed and truncated (to say, a $500 \mathrm{~ms}$ time frame), in order to obtain the room impulse response. The channels so measured have been used in this thesis work for the simulation studies.

The experimental set up was the same as in the simulation studies, with $L=3$, $M=2, N_{\mathrm{c}}=13871$ and $N_{\mathrm{r}}=16643$ and a sampling frequency of $44.1 \mathrm{kHz}$. The $T_{60}$ of the room was $245 \mathrm{~ms}$. The separation between two adjacent loudspeakers was $24 \mathrm{~cm}$ and that between the two microphones was $12 \mathrm{~cm}$. The average loudspeaker to microphone distance was $37 \mathrm{~cm}$. The unreshaped channel had an nPRQ measure of 8.41. The pre-echo, early reflection and late reverberation regions were set to $22 \mathrm{~ms}, 24 \mathrm{~ms}$ and $199 \mathrm{~ms}$ respectively. The performance metrics CDR and nPRQ were used in the study.

In the shaping experiment, the shaping algorithm was applied to the channel measured with the loudspeakers to design the shaping filters. The designed filters were applied to the chirp signal before sounding the loudspeakers. The loudspeak- 


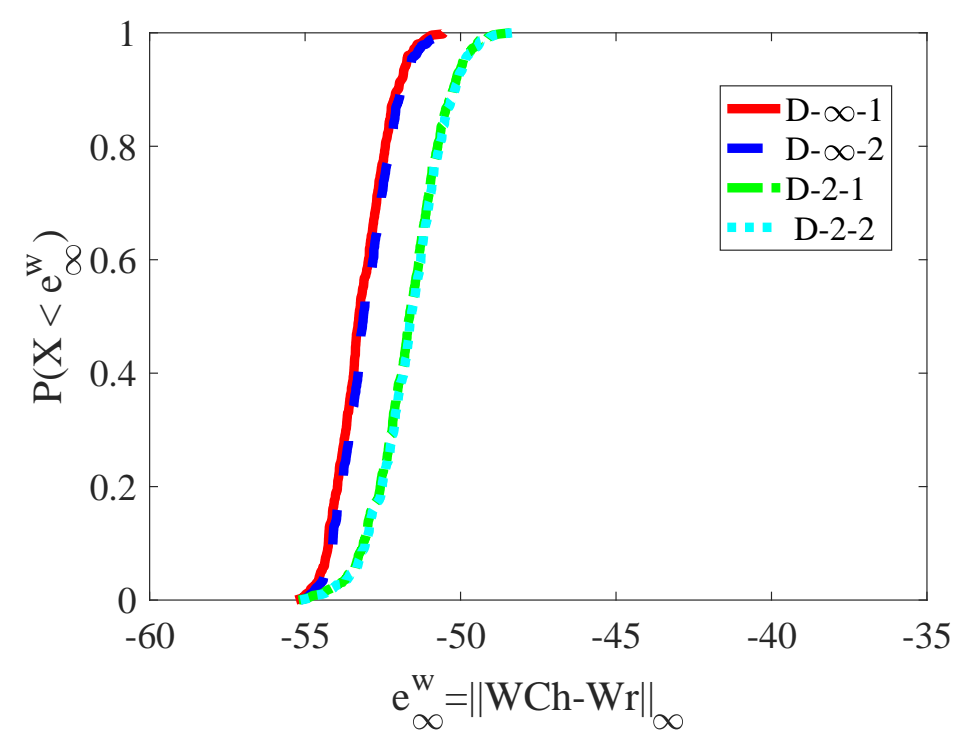

Figure 5.11: Empirical CDF of the $\ell_{\infty}$ errors at the original position and 500 perturbed positions at $1 \mathrm{~cm}$ from the original position using the robust approach of design over the original position and 15 perturbed positions at $1 \mathrm{~cm}$ from the original position.

ers were again played and the signal received at the microphones were measured. The results using D-2-1 and D- $\infty-1$ are shown here. The results with D-2-2 and D- $\infty-2$ were also similar. From Figure 5.12 and Figure 5.13, it can be seen that good shaping has been achieved with low late reverberation and pre-echo. The nPRQ values were found to be 4.17 and 4.81 respectively with D-2-1 and D- $\infty-1$. A good cross-talk cancelation of around $-35 \mathrm{~dB}$ was also achieved as can be seen from Figure 5.12 and Figure 5.13. The algorithms were found to be computationally fast, with a computation time of $8 \mathrm{~s}$ (D-2-1) and $25 \mathrm{~s}(\mathrm{D}-\infty-1)$. This validates the applicability of the impulse response shaping algorithms developed in this work provide to provide realtime implementations.

In order to assess the perceptual quality of the shaped responses, an informal listening test was conducted. The loudspeakers were fed with a chirp signal sampled at $44.1 \mathrm{kHz}$ and the signal received at the microphones were recorded. These signals were used to compute the channels $c_{m l}$ as explained in earlier paragraphs. The shaping filters were then computed using D- $\infty-1$ algorithm. Chirp signals sampled at $44.1 \mathrm{kHz}$ were then generated, convolved with the shaping pre-filters 

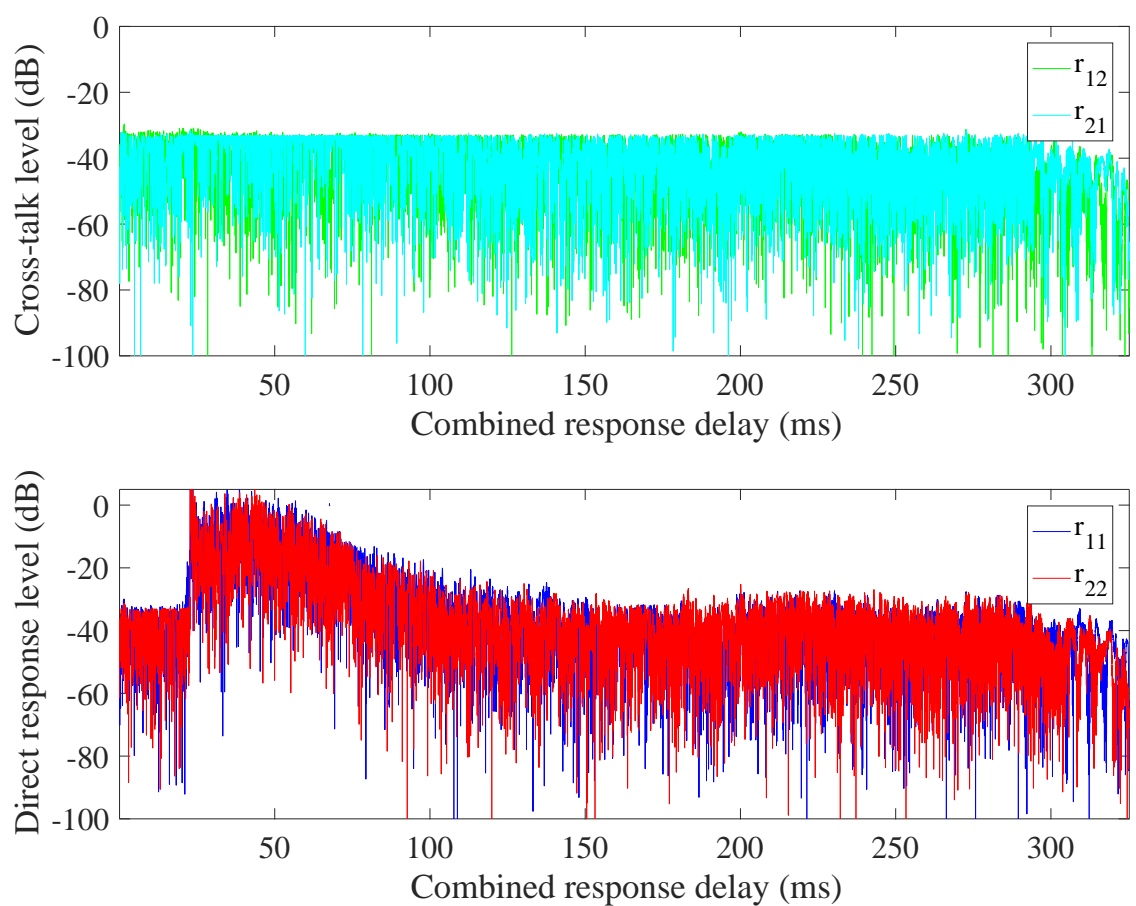

Figure 5.12: a) Cross-talk b) Shaped direct responses obtained with D- $\infty$-1 using the VACS system.

and fed to the loudspeakers. The signals received at the microphones were played back for listening. It was observed that the effect of shaping was empirically very clear. The selective suppression of reverberation in the various response segments (pre-echo, early and late reverberations) was apparent. Normally in listening experiments based on channel inversion, the suppression is so complete that the response presents as though it were totally anechoic. By contrast, using shaping, the reverberation was sustained for a short time, which sounded pleasing to the ear.

This experiment was repeated with the other shaping algorithms developed, viz., D- $\infty-2$, D-2-1 and D-2-2. It was observed that the results obtained with the $\ell_{\infty}$ algorithms (D- $\left.\infty-1, D-\infty-2\right)$ were superior in suppression of the late reverberation than the $\ell_{2}$ algorithms (D-2-1, D-2-2).

Multi-channel inversion was also implemented to compare the shaping performance with inversion. The result obtained in this case is shown in Figure 5.14. It 

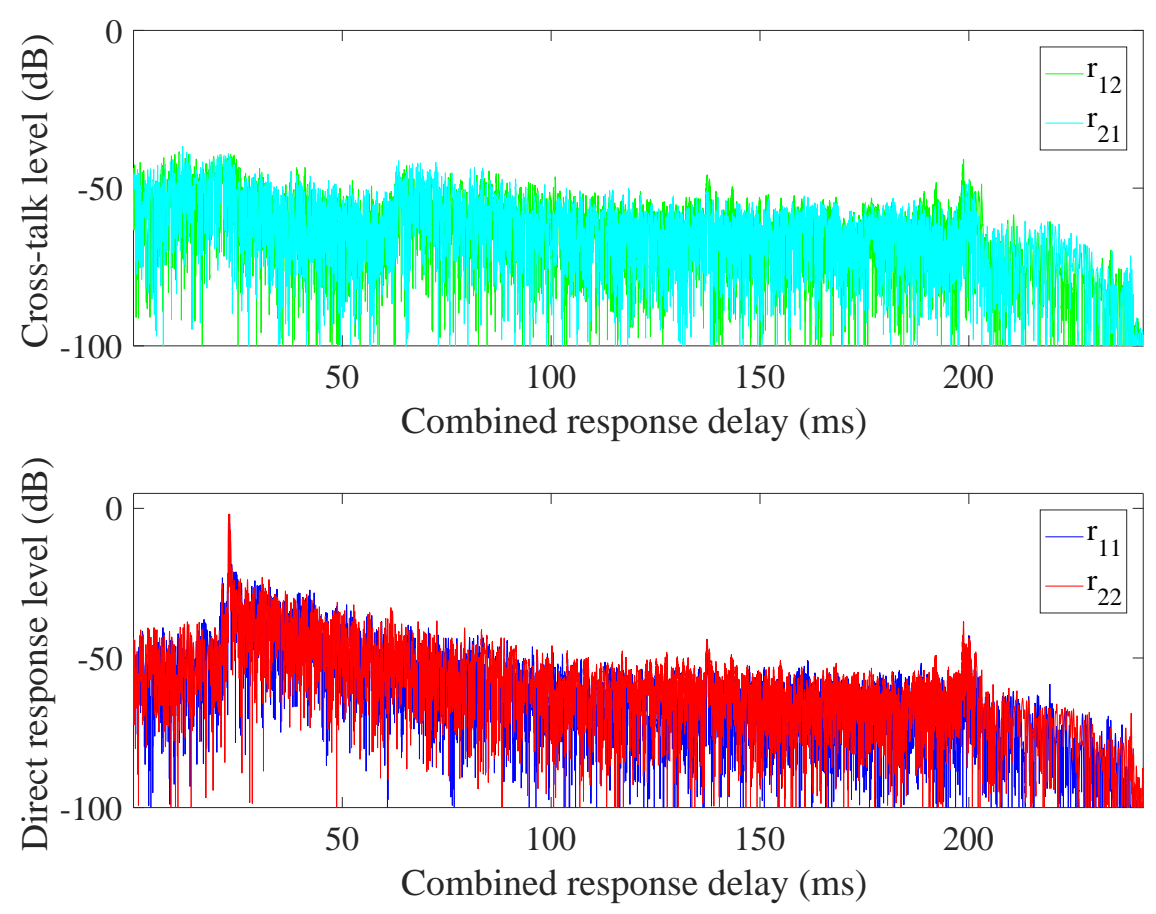

Figure 5.13: a) Cross-talk b) Shaped direct responses obtained with D-2-1 using the VACS system.

can be seen that inversion provides better performance than shaping with a CDR of $-40 \mathrm{~dB}$. This matches with the theory that inversion provides better performance than the relaxed problem of shaping for when the design and test conditions are the same.

\subsubsection{Robustness to atmospheric conditions}

The robustness of the shaping filters to changes in atmospheric conditions such as temperature and humidity was studied using the VACS experimental setup. The channels were measured every hour during a 24-hour period and the filters designed initially were applied before the loudspeakers and the microphone outputs were recorded. The design was performed at midnight and the set up was left to run till $11 \mathrm{pm}$ the next day. It was observed that the CDR values were almost steady for the 24-hour measurement period with only a small observed fluctuation as can be seen in Figure 5.15. The algorithms were also found to perform good shaping with a slightly increased amount of late reverberation at higher 

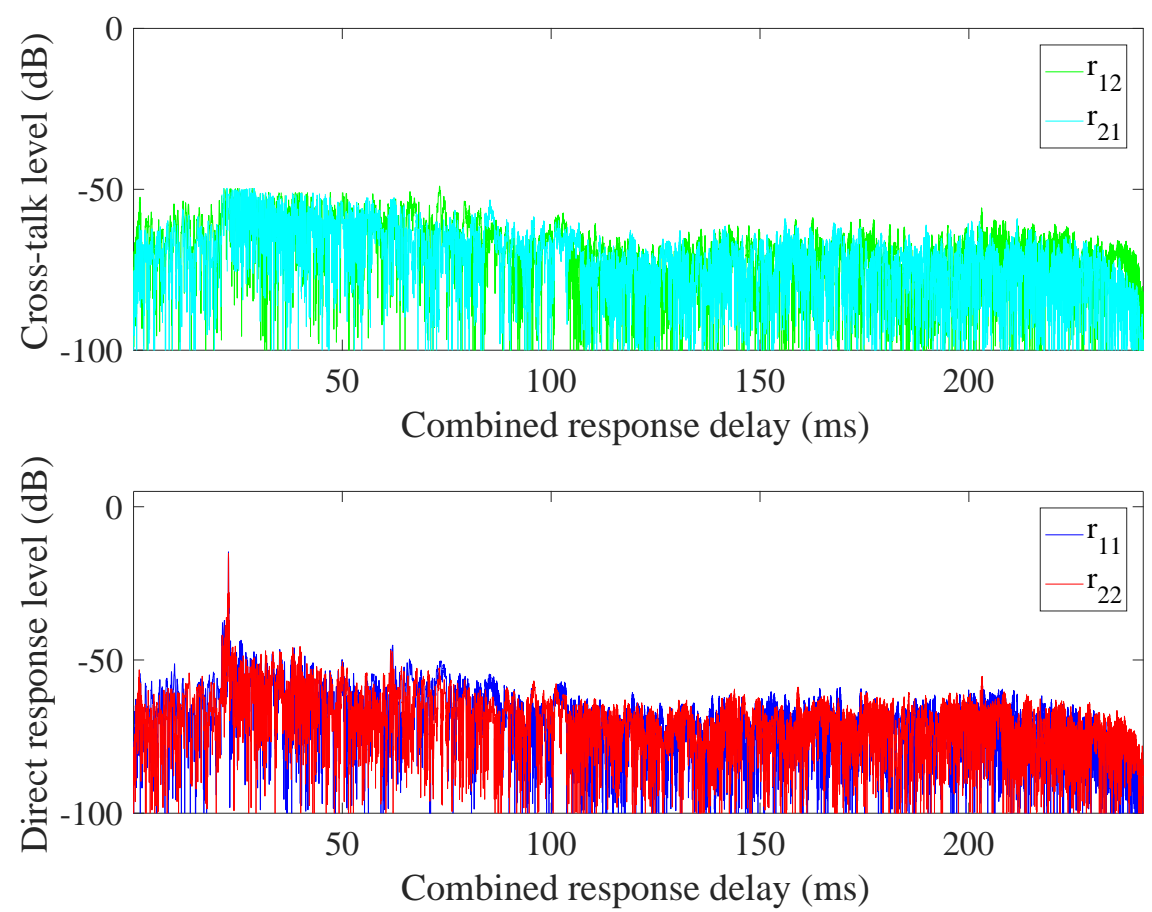

Figure 5.14: a) Cross-talk b) Shaped direct responses obtained with multichannel inversion using the VACS system.

temperatures, especially close to noon. At noon, the nPRQ values were found to be 5.43 and 5.69 with $\mathrm{D}-2-1$ and D- $\infty-1$. These results indicate that the shaping algorithms developed show some inherent robustness to changes in atmospheric conditions, though not explicitly designed for this robustness. The inverse filters, on contrast, were found to be more sensitive and CDR was found to fluctuate by large amounts compared to shaping as shown in Figure 5.15. The pre-echo and reverberations were also found to be elevated at measurements other than the designed conditions. This again highlights the advantage of shaping over inversion.

This experimental validation is not a complete study, but validates the scope for developing a shaping system based on these algorithms. There is scope for various experimental studies with this setup; for example, analysis of robustness to change in microphone positions, effect of the presence and absence of a moving object in the room, opening and closing of the door etc. We leave these experiments for future work. 


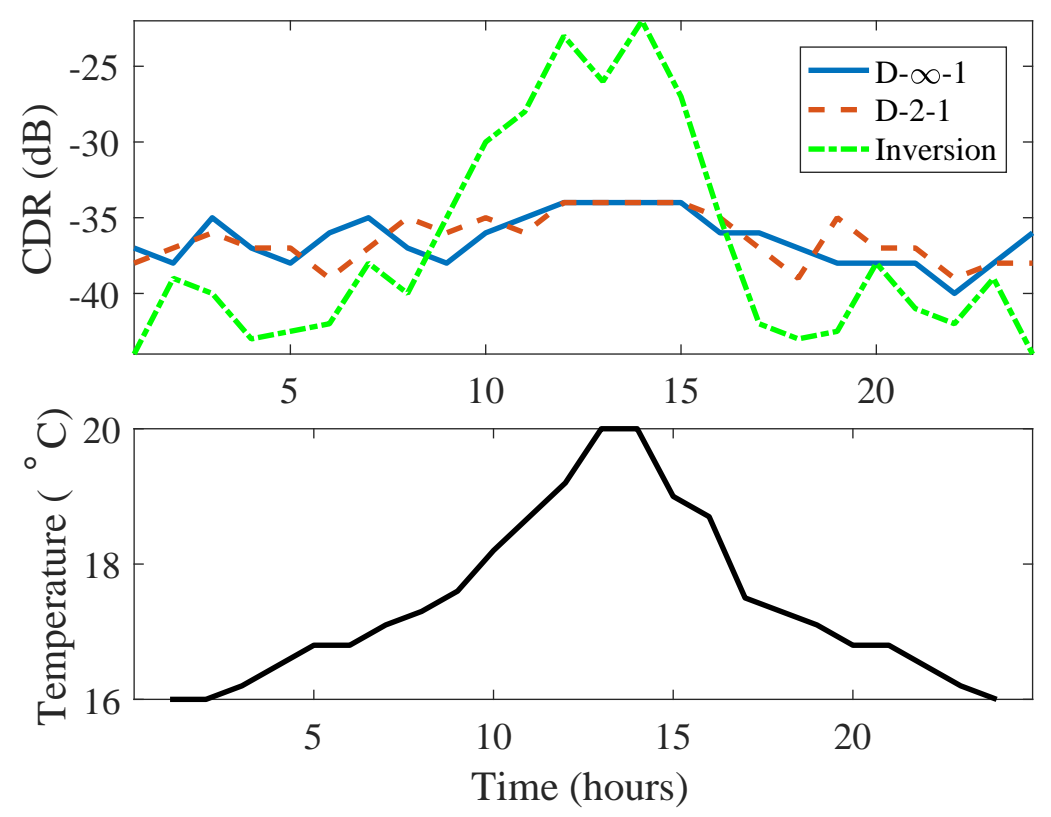

Figure 5.15: CDR values measured over a 24-hour period.

\subsection{Contributions from this chapter}

One of the contributions of this chapter is the application of the DALM algorithm for solving $\ell_{1}$ regularized $\ell_{2}$ minimization problems (D-2-1) to the impulse response shaping problem. Good cross-talk cancelation performance has been achieved with fast computational time, both in simulation and experimental studies, that indicates its applicability to a real time room response shaping system. Other developments from this chapter are extension of the DALM algorithm to various combinations of regularizers and fidelity factors, which can be advantageous for the shaping problem. The new algorithms developed are D-2-2, D- $\infty-1$ and D- $\infty$ 2. The development of $\mathrm{D}-\infty-1$ and $\mathrm{D}-\infty-2$ are the major contributions from this chapter since these algorithms constrain the maximum value of error between the estimated and expected responses, which is the ideal choice for achieving optimal shaping. The new algorithms were implemented in the form of an FAO for added memory advantages.

The efficacy of the algorithms in shaping was verified using the simulation results that showed a consistent CDR of $-58 \mathrm{~dB}$ and experimental results with a CDR of around $-35 \mathrm{~dB}$ for all the four combinations. D- $\infty-1$ or $\mathrm{D}-\infty-2$ can be 
chosen if stringent constraints are to be imposed, while D-2-1 or D-2-2 can be chosen if computational speed is more relevant than strictness of constraints.

Other contributions are the application of the DALM algorithms to the shaping over multiple microphone positions in order to achieve spatial robustness to change in microphone positions. It has been brought out from the simulation studies that the formulation is very robust along with providing good cross-talk cancelation with the desired shaping. 


\section{Chapter 6}

\section{Joint Time and Frequency Shaping}

\subsection{Background}

In the previous chapter, fast algorithms based on DALM for multi-channel acoustic impulse response shaping were presented. The goal was to design pre-filters so that the combined response of the pre-filters and room impulse response has low cross-talk, and reduced reverberation. In addition, the algorithms were modified to include a design over multiple position to ensure that the resulting shaped responses were robust against measurement errors or movement of the sensors. The limitations of these earlier approaches are that they are concerned only with the time domain and did not accommodate explicit control of the combined frequency domain. Accordingly, the frequency response sometimes exhibited peaks or troughs, rather than a flat response across the frequency spectrum of operation, and such fluctuations are clearly undesirable. A frequency domain equalisation component is an obvious improvement. In this chapter, we use a proximal projection to achieve the frequency equalisation. This results in a modification to our original fast DALM based shaping algorithm to incorporate the additional frequency projection step.

An approach for combined frequency control is explained in [28] in which the frequencies producing high peaks in the final responses are found and the peaks are reduced by minimizing a norm of those frequency samples. This is computationally slow since it requires application of the solution algorithm twice; once to 
obtain time shaping, then finding the violated frequencies and a consecutive run to attain frequency control as well.

In the approach presented in this chapter, the DALM iterations and frequency control are performed together in an alternating fashion until convergence. Computationally fast variants of this algorithm are also presented which effectively work in the same fashion as in [28] but enable implementation of joint time and frequency shaping approach in real time due to their computational efficiency. This computational efficiency is achieved by running the time shaping algorithm for only a few iterations and then alternating time and frequency projection. Since the DALM iterations execute very fast and progress towards the solution quickly, running few such iterations initially doesn't affect the computational speed much and moreover, helps in faster convergence in the later steps when the time and frequency projection steps are run alternatively. The contents of this chapter are extensions of the paper [116].

\subsection{Problem Formulation}

It has been shown in [35] that the DALM algorithm can be considered as a proximal minimization iteration. Considered from this point of view, frequency domain equalization can be considered as an additional proximity operation in the iteration: proximity being a generalization of the familiar concept of projection $[117,118]$. To $(4.8)$, we can add a constraint on the magnitude of a given component of the combined frequency response. An upper bound on this magnitude is a convex constraint; we do not attempt a lower bound, which would be nonconvex. The GIR is found as $C h$ (as in Figure 4.1), which can also be written as $I \otimes \mathcal{C} h$, where $I$ is the identity matrix, $\mathcal{C}$ contains the Toeplitz matrices (representing convolution) corresponding to each channels and $\otimes$ is the Kronecker product. The frequency response can therefore be written as $I \otimes F \mathcal{C} h$ where $F$ is the DFT matrix. The combined transfer function is given by $I \otimes F$. The response for a particular source-to-sensor transfer and for a particular frequency can be obtained using $g^{*} h$ where superscript $*$ represents the adjoint (complex transpose) and $g^{*}$ is a particular row of $(I \otimes F) \mathcal{C}$. The projection operation is performed for all positive frequencies found in this manner successively. This is because performing the projection at all frequencies jointly is computationally prohibitive and spectral flatness can be obtained by successively performing this projection for different values of $g$, one for each frequency. In the case of a hard constraint, the proximity 
operation becomes a projection operation: the problem is thus to find the closest (in Euclidean norm sense) value to a given impulse response $h_{0}$ that does not exceed a certain magnitude in the frequency response when the impulse response is combined with a second filter.

The required operation can thus be expressed as

$$
\begin{array}{ll}
\min _{h} & \left\|h-h_{0}\right\|_{2}^{2} \\
\text { subject to } & \left|g^{*} h\right| \leq \gamma
\end{array}
$$

This is the problem that we solve in the following subsection.

\subsubsection{Projection algorithm}

Since there are $M$ direct responses for a shaping system with $M$ microphones, we define the $M \times 1$ vectors

$$
\begin{aligned}
& g_{\mathrm{r}}=\Re(g) \\
& g_{\mathrm{i}}=\Im(g)
\end{aligned}
$$

where $\Re()$ denotes the real part and $\Im()$ denotes the imaginary part. Now we can define the rank 2 matrix

$$
P=g_{\mathrm{r}} g_{\mathrm{r}}^{*}+g_{\mathrm{i}} g_{\mathrm{i}}^{*}=G G^{*}
$$

where $G$ is the $M \times N$ matrix given by

$$
G=\left[\begin{array}{ll}
g_{\mathrm{r}} & g_{\mathrm{i}}
\end{array}\right]
$$

In this case, $N=2$. The following condition and derivations are also valid for $N$ greater than 2 .

The constraint in the optimization problem (6.1) can now be rewritten as

$$
h^{*} P h \leq \gamma^{2}
$$

The Lagrangian of the constrained minimization with Lagrange multiplier $\mu$ is therefore

$$
L=h^{*} h+h_{0}^{*} h_{0}-2 h_{0}^{*} h-\mu\left(\gamma^{2}-h^{*} P h\right)
$$

Equating the partial derivative of $L$ with respect to $h$ to zero gives

$$
\frac{\partial L}{\partial h}=2\left(I_{\mathrm{M}}+\mu P\right) h-2 h_{0}=0
$$


where $I_{\mathrm{M}}$ is the $M \times M$ identity matrix. So we have

$$
h=\left(I_{\mathrm{M}}+\mu P\right)^{-1} h_{0}
$$

Combining (6.6) and (6.9) gives the equation to find the value of Lagrange multiplier $\mu$ as

$$
h_{0}^{*}\left(I_{\mathrm{M}}+\mu P\right)^{-1} P\left(I_{\mathrm{M}}+\mu P\right)^{-1} h_{0}=\gamma^{2}
$$

which does not seem particularly easy to solve for $\mu$.

However, consider the Eigen decomposition of $P$ :

$$
P=\left[\begin{array}{ll}
V & U
\end{array}\right]\left[\begin{array}{cc}
D & 0 \\
0 & 0
\end{array}\right]\left[\begin{array}{c}
V^{*} \\
U^{*}
\end{array}\right]=V D V^{*}
$$

where $V$ are the eigenvectors corresponding to non-zero eigenvalues in the diagonal matrix $D$, and $U$ are the eigenvectors corresponding to zero eigenvalues. The matrix $\mathrm{P}$ is Hermitian and so $\left[\begin{array}{ll}V & U\end{array}\right]$ is orthonormal. This means that

$$
\left(I_{\mathrm{M}}+\mu P\right)^{-1}=V\left(I_{\mathrm{N}}+\mu D\right)^{-1} V^{*}
$$

and so

$$
\begin{aligned}
& h_{0}^{*}\left(I_{\mathrm{M}}+\mu P\right)^{-1} P\left(I_{\mathrm{M}}+\mu P\right)^{-1} h_{0} \\
& =h_{0}^{*} V\left(I_{\mathrm{N}}+\mu D\right)^{-1} D\left(I_{\mathrm{N}}+\mu D\right)^{-1} V^{*} h_{0} \\
& =h_{0}^{*} V D^{\prime} V^{*} h_{0} \\
& =\operatorname{tr}\left(D^{\prime} V^{*} h_{0} h_{0}^{*} V\right) \\
& =\sum_{i} d_{i}^{\prime} f_{i}
\end{aligned}
$$

where the $i$ th element of diagonal matrix $D^{\prime}$ is given from the $i$ th element $d_{i}^{\prime}$ of diagonal matrix $D$ by

$$
d_{i}^{\prime}=\frac{d_{i}}{\left(1+\mu d_{i}\right)^{2}}
$$

and $f_{i}$ is the $i$ th diagonal element of the matrix $V^{*} h_{0} h_{0}^{*} V$.

This means that the eigenvectors $U$ are irrelevant to the solution. The relevant matrices $V$ and $D$ can be straightforwardly computed from the singular value decomposition of $G$. Alternatively, and more efficiently, $V$ and $D$ can be computed 
from the very small eigen decomposition $G^{*} G=F E F^{*}$ :

We seek the matrix $B$ such that $V=G B$, so that

$$
V^{*} V=B^{*} G^{*} G B=I
$$

If we define $B=F E^{-\frac{1}{2}}$, then

$$
\begin{aligned}
B^{*} G^{*} G B & =B^{*} F E F^{*} B=E^{-\frac{1}{2}} F^{*} F E F^{*} F E^{-\frac{1}{2}} \\
& =E^{-\frac{1}{2}} E E^{-\frac{1}{2}}=I
\end{aligned}
$$

as required. Using this definition of $B$, we find that

$$
\begin{aligned}
V D V^{*} & =G B D B^{*} G^{*}=G F E^{-\frac{1}{2}} D E^{-\frac{1}{2}} F^{*} G^{*} \\
& =G F F^{*} G^{*}=G G^{*}=A
\end{aligned}
$$

provided that $D=E$.

Thus the required value of $\mu$ is the solution to the equation

$$
\sum_{i=1}^{N} \frac{f_{i} d_{i}}{\left(1+\mu d_{i}\right)^{2}}=\gamma^{2}
$$

For the case that $N=2$, this equation can be written as

$$
f_{1} d_{1}\left(1+\mu d_{2}\right)^{2}+f_{2} d_{2}\left(1+\mu d_{1}\right)^{2}=\gamma^{2} 2\left(1+\mu d_{2}\right)^{2}\left(1+\mu d_{1}\right)^{2}
$$

and hence as the quartic

$$
\begin{aligned}
& \gamma^{4} d_{1}^{2} d_{2}^{2} \mu^{4}+2 \gamma^{2} d_{1} d_{2}\left(d_{1}+d_{2}\right) \mu^{3} \\
& +\left(\gamma^{2}\left(d_{1}^{2}+d_{2}^{2}+4 d_{1} d_{2}\right)-f_{1} d_{1} d_{2}\left(d_{1}+d_{2}\right)\right) \mu^{2} \\
& +\left(2 \gamma^{2}\left(d_{1}+d_{2}\right)-2 d_{1} d_{2}\left(f_{1}+f_{2}\right)\right) \mu \\
& +\left(\gamma^{2}-f_{1} d_{1}-f_{2} d_{2}\right)=0
\end{aligned}
$$

which can be simply solved analytically for its single real positive root [119]. After finding $\mu$, the solution $h$ can be found from (6.9) and (6.12) as

$$
h=V\left(I_{\mathrm{N}}+\mu D\right)^{-1} V^{*} h_{0}
$$




\subsubsection{Special Cases}

If the vector $g$ is purely real or purely imaginary, the equation to solve for $\mu$ becomes a quadratic. This gives two solutions for $\mu$ and the positive root can be chosen. The derivation for the purely real case is detailed here.

If $g$ is purely real, $g_{\mathrm{i}}=0$. Therefore, $G=\left[\begin{array}{ll}g_{\mathrm{r}} & 0\end{array}\right]$ for $N=2$. Now,

$$
G^{*} G=\left[\begin{array}{cc}
g_{\mathrm{r}}^{*} g_{\mathrm{r}} & 0 \\
0 & 0
\end{array}\right]
$$

The eigen decomposition of $G^{*} G$ gives the eigenvalues $g_{\mathrm{r}}^{*} g_{\mathrm{r}}$ and 0 . The eigenvector corresponding to the non-zero eigenvalue is $\left[\begin{array}{ll}1 & 0\end{array}\right]^{\mathrm{T}}$. Hence, in this case, we have only one $v_{1}=\left[\begin{array}{ll}1 & 0\end{array}\right]^{\mathrm{T}}$ and $d_{1}=g_{\mathrm{r}}^{*} g_{\mathrm{r}}$. Therefore, (6.19) becomes

$$
\frac{f_{1} d_{1}}{\left(1+\mu d_{1}\right)^{2}}=\gamma^{2}
$$

which on simplification becomes a quadratic in $\mu$ given by

$$
d_{1}^{2} \gamma^{2} \mu^{2}+2 d_{1} \gamma^{2} \mu+\left(\gamma^{2}-f_{1} d_{1}\right)=0
$$

The equation for finding the solution $h$ reduces to

$$
h=\frac{v_{1} v_{1}^{*}}{1+\mu d_{1}} h_{0}
$$

In the case that $g$ is purely imaginary, we have $v_{1}=\left[\begin{array}{ll}1 & 0\end{array}\right]^{\mathrm{T}}$ and $d_{1}=g_{\mathrm{i}}^{*} g_{\mathrm{i}}$. The rest of the derivation steps are similar to that of the $g$ being purely real case.

\subsubsection{Time and frequency shaping algorithm}

The joint time and frequency shaping problem can be written as an optimization problem of the form

$$
\begin{aligned}
& \min _{h}\|W(C h-r)\|_{\mathrm{u}}+\lambda\|h\|_{\mathrm{v}} \\
& \text { subject to } \quad\left|g_{k}^{*} h\right| \leq \gamma \quad k \in\left\{1 \ldots M N_{\mathrm{r}}\right\}
\end{aligned}
$$

where $g_{k}^{*} h$ is the response at each of the $N_{\mathrm{r}}$ frequencies for $M$ desired responses. Here, $N_{\mathrm{r}}$ and $M$ are defined as in Section 4.1. The problem (6.26) is split into two sub-problems : (4.8) and (6.1) which are solved one after the other to achieve 
a feasible solution. This is implemented as a frequency projection step after one DALM iteration till convergence. However, the frequency response that is flattened by a projection step may be disturbed by the following DALM iteration. In addition, the projection step is slower than the DALM iteration since it sequentially works through all the constrained frequencies. Therefore, a computationally more efficient approach is to perform a projection step after several DALM iterations. This approach results in a reasonable time and frequency domain performance, in addition to faster computational speed as can be seen in the experimental results detailed in the following section.

\subsection{Robust time and frequency shaping algorithm}

Spatial robustness to errors in microphone positions can be achieved by a design over multiple microphone positions as explained in Section 5.6. In the case of joint time and frequency shaping, the DALM iterations for achieving time shaping are performed similar to the approach explained in Section 5.6 but the projection step for frequency shaping is performed over all frequencies of all perturbed copies of the original channel response. This is achieved by performing the projection of the sample values of all perturbed copies at a particular frequency and then sequentially moving onto the next frequency. This process ensures that the desired frequency shaping is achieved at the original and at all the perturbed positions.

In the case of joint time and frequency shaping algorithm using D-2-1 or D-2-2 formulations of DALM, the optimization problem can be stated as

$$
\min _{h} \frac{1}{2} \sum_{n=1}^{N_{\mathrm{p}}+1}\left\|W C^{n} h-W r\right\|_{2}^{2}+\frac{1}{\mathrm{v}} \lambda\|h\|_{\mathrm{v}}
$$

for $\mathrm{v} \in\{1,2\}$ and $k \in\left[1,\left(N_{\mathrm{p}}+1\right) M N_{\mathrm{r}}\right]$ where $N_{\mathrm{p}}$ is the number of perturbations and $C^{n}$ are the channel matrices corresponding to each perturbation, as in Section 5.6. When $\mathrm{D}-\infty-1$ or $\mathrm{D}-\infty-2$ are used, the optimization problem can be stated as

$$
\begin{aligned}
& \min _{h}\|W \breve{C} h-W r\|_{\infty}+\frac{1}{\mathrm{v}} \lambda\|h\|_{\mathrm{v}} \\
& \text { subject to }\left|g_{k}^{*} h\right| \leq \gamma
\end{aligned}
$$




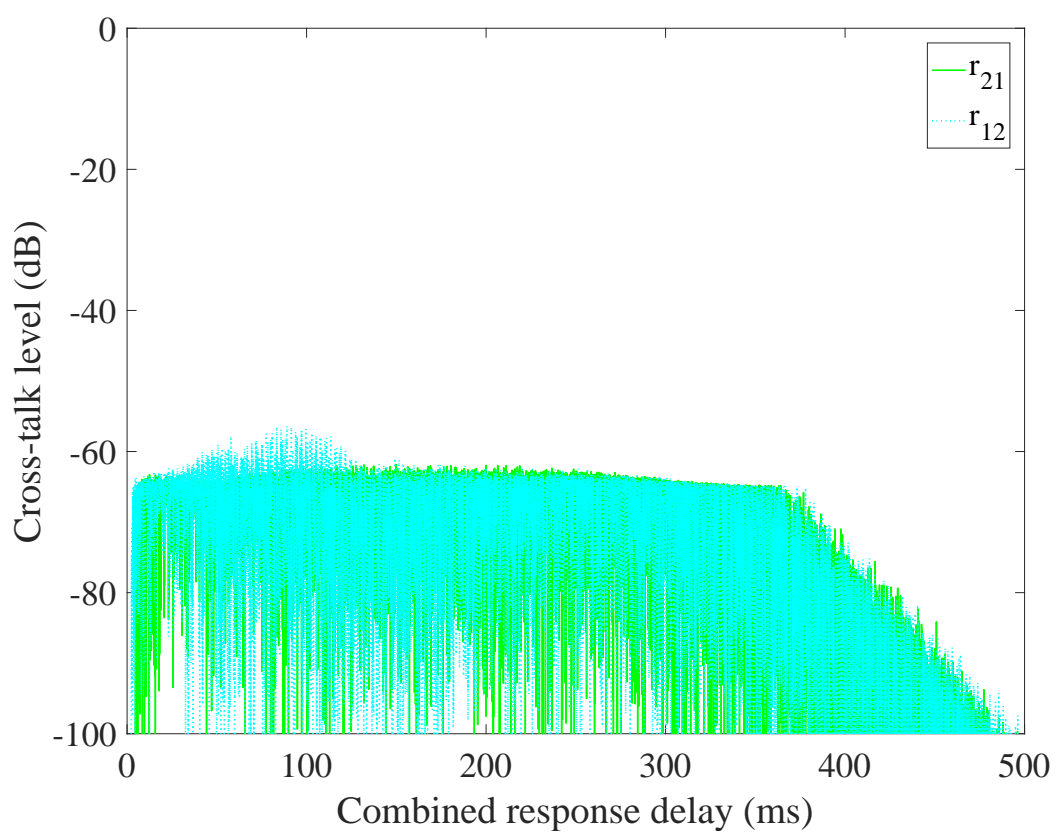

Figure 6.1: The two cross-talk responses $r_{12}$ and $r_{21}$ obtained with Algorithm A. (Algorithm A works by alternating DALM iterations and frequency projection one after the other till convergence.)

where

$$
\breve{C}=\left[\left(C^{1}\right)^{\mathrm{T}} \ldots\left(C^{n}\right)^{\mathrm{T}} \ldots\left(C^{N_{\mathrm{p}}+1}\right)^{\mathrm{T}}\right]^{\mathrm{T}}
$$

It can be seen that the optimization problem becomes large for $\mathrm{D}-\infty-1$ or $\mathrm{D}$ $\infty-2$ since both time and frequency shaping each take $N_{\mathrm{p}}+1$ times the original computational time. Thus some approximations are used in the original shaping algorithm to achieve computational speed, which are explained in the following section.

\subsection{Simulation results}

Experimental simulation studies were performed using the channels measured in the same room as in Section 5.7 using the same set up as in Figure 5.1 with $L=3$ loudspeakers, $M=2$ microphones, channel length $N_{\mathrm{c}}=10000$ and $N_{\mathrm{r}}=32768$ and a sampling frequency of $44.1 \mathrm{kHz}$. The $T_{60}$ of the room was $245 \mathrm{~ms}$ and the Spectral Flatness Measure (SFM) $[106,107]$ was 0.57 . The pre-echo, early reflection 


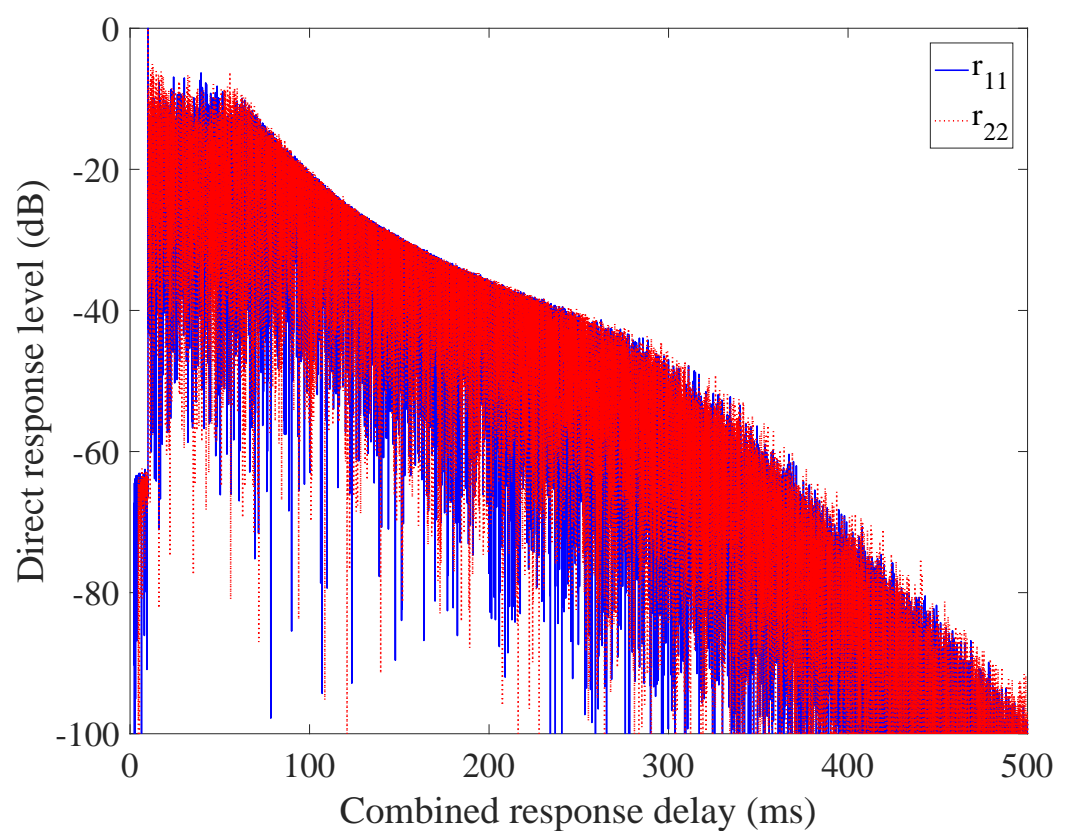

Figure 6.2: The two direct responses $r_{11}$ and $r_{22}$ obtained with Algorithm A (by alternating DALM iterations and frequency projection steps till convergence).

and late reverberation regions were set as $10 \mathrm{~ms}, 40 \mathrm{~ms}$ and $200 \mathrm{~ms}$ respectively as in Section 5.7. The performance metrics used in this chapter are CDR, nPRQ and SFM which are defined in Section 4.4. Here, SFM quantifies the frequency flattening performance and nPRQ quantifies the time shaping performance. The parameter $\gamma$ was set to be the 85th percentile value of the unconstrained solution. Decreasing $\gamma$ obviously improves spectral flatness, but at the cost of greater crosstalk.

The joint time and frequency shaping algorithm, proposed in this chapter, that uses a combination of the projection algorithm and the DALM algorithm was tested using the above mentioned channels. This was done by performing one frequency projection step after one DALM iteration (Algorithm A). The $\ell_{1}$ regularized $\ell_{\infty}$ DALM (D- $\left.\infty-1\right)$ formulation is used in this set of results. Figure 6.1 shows the cross-talk response showing a cross-talk cancelation of about $-56 \mathrm{~dB}$. The direct responses shown in Figure 6.2 show that the peak (direct path) is approximately $20 \mathrm{~dB}$ above the smaller values and the response decays rapidly after the allowed early reflections, thus reducing the late reverberation. Thus, a CDR 


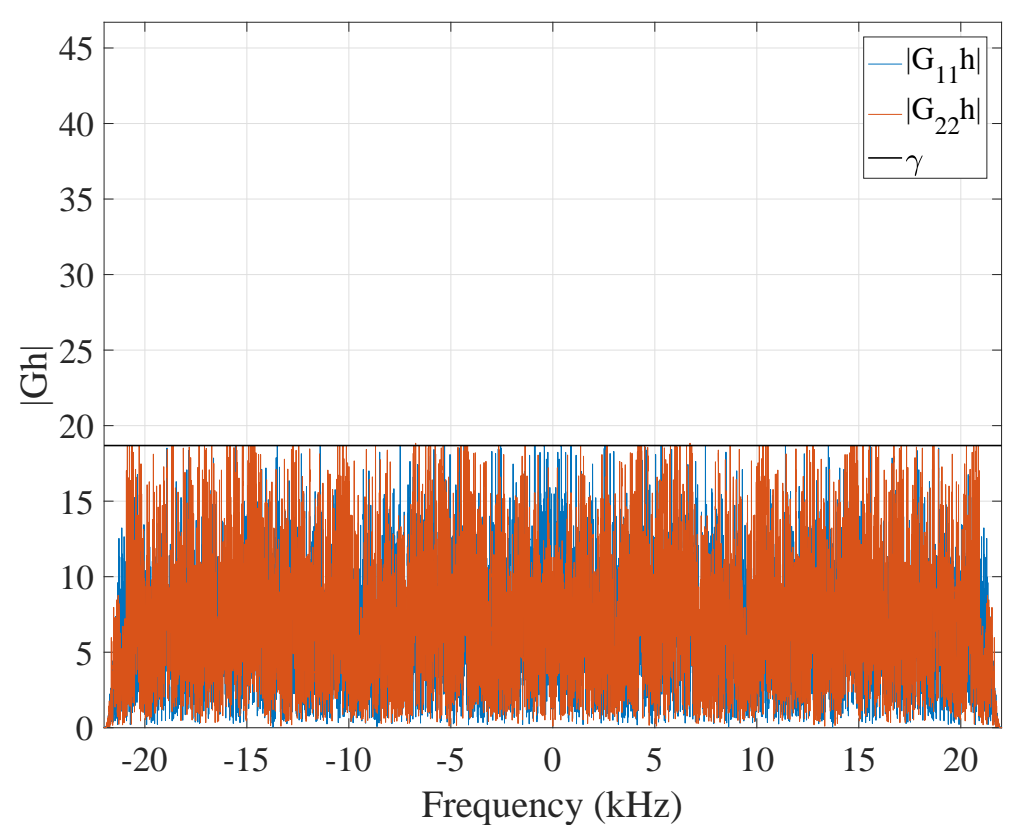

Figure 6.3: Frequency responses of the two channels obtained with Algorithm A (by alternating DALM iterations and frequency projection steps till convergence).

of around $-56 \mathrm{~dB}$ was achieved. The frequency response of the direct responses shown in Figure 6.3 shows that all the frequencies have been modified to obey the frequency constraint. The spectral flatness (SFM) was found to be 0.835. The CDF of the frequency responses are shown in Figure 6.4. Thus, joint time and frequency shaping was achieved using this algorithm. In addition, the final objective function value was found to be 0.525 which is very close to the frequency unconstrained D- $\infty-1$ solution of 0.51 . These results are summarized in Table 6.1 and demonstrate that additional frequency constraint has not significantly degraded the time domain performance.

A direct comparison with single channel time-frequency shaping in [120] is not possible without using the same channel, but the SFM results are quite similar. (SFM in that work increased from 0.52 for raw channel to $0.74-0.8$ after shaping.) The computation time using Algorithm A was found to be prohibitive (5 days, 3982 combined iterations). This is because the projection step works on each frequency constraint sequentially and is thus computationally very slow. Therefore, a faster version of this algorithm is desired for practical applications. 


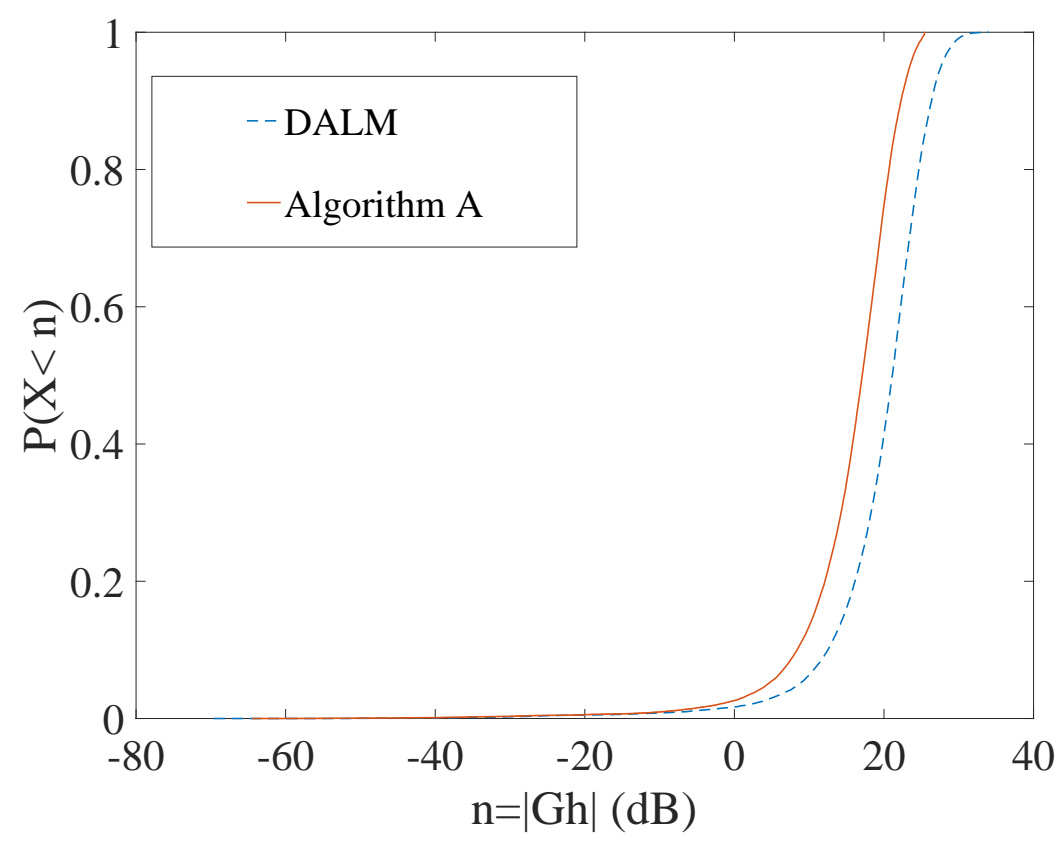

Figure 6.4: Cumulative density function of the frequency responses obtained with frequency unconstrained DALM and Algorithm A (by alternating DALM iteration and frequency projection steps till convergence).

Table 6.1: Performance of the frequency projection algorithms in shaping. Performance metrics CDR, CPU time, SFM and nPRQ are defined as in Section 4.4. Objective function value is the final value obtained for the objective in (6.26) with $u=\infty, v=1$.

\begin{tabular}{cccccc} 
Algorithm & CDR & CPU Time & Objective & SFM & nPRQ \\
& $(\mathrm{dB})$ & $(\mathrm{s})$ & function & & $(\mathrm{dB})$ \\
\hline D- $\infty-1$ & -58 & 85 & 0.51 & 0.81 & 4.31 \\
\hline Algorithm A & -56 & 438048 & 0.525 & 0.835 & 2.61 \\
\hline Algorithm B $\left(N_{\text {iter }}=100\right)$ & -49 & 3336 & 0.674 & 0.823 & 4.52 \\
\hline Algorithm B $\left(N_{\text {iter }}=500\right)$ & -44 & 1498 & 0.702 & 0.818 & 4.49 \\
\hline Algorithm C & -39 & 368 & 0.758 & 0.854 & 3.72 \\
\hline
\end{tabular}




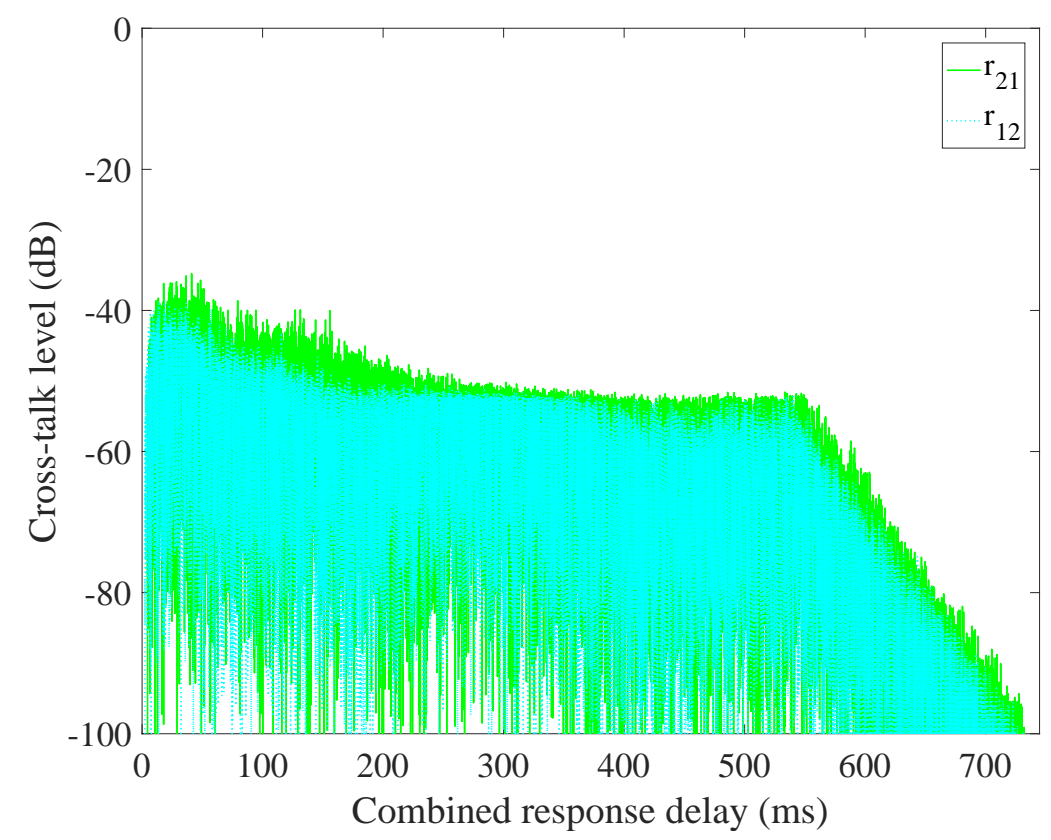

Figure 6.5: The two cross-talk responses obtained with Algorithm C (Fast version of joint time and frequency shaping algorithm).

A faster version of Algorithm A, which we name as Algorithm B, proceeds as several iterations of the original D- $\infty-1$ followed by alternating projection and DALM steps until convergence. Proper choice of the number of DALM iterations before the projection step, which obviously affects the computational speed, is important. It was found that a reasonable choice of this parameter $\left(N_{\text {iter }}\right)$ for this data is 100 , given that the frequency unconstrained algorithm takes 1572 iterations to converge to the solution. This algorithm was found to be about 60 times faster than Algorithm A, in addition to providing a reasonable CDR of -49 dB. Given that this algorithm works, the number of the DALM iterations before a projection step was varied. It was found that Algorithm B converges faster as $N_{\text {iter }}$ increases. These results are summarised in Table 6.1. Though Algorithm B is faster than Algorithm A, it is still computationally slow.

For both algorithms $\mathrm{A}$ and $\mathrm{B}$, the stopping criterion was simply that the change between successive iterations was small. The original shaping problem and the frequency domain constraints are all convex, so we expect that algorithms $\mathrm{A}$ and $\mathrm{B}$ will eventually converge to the same solution. However, a computationally faster 


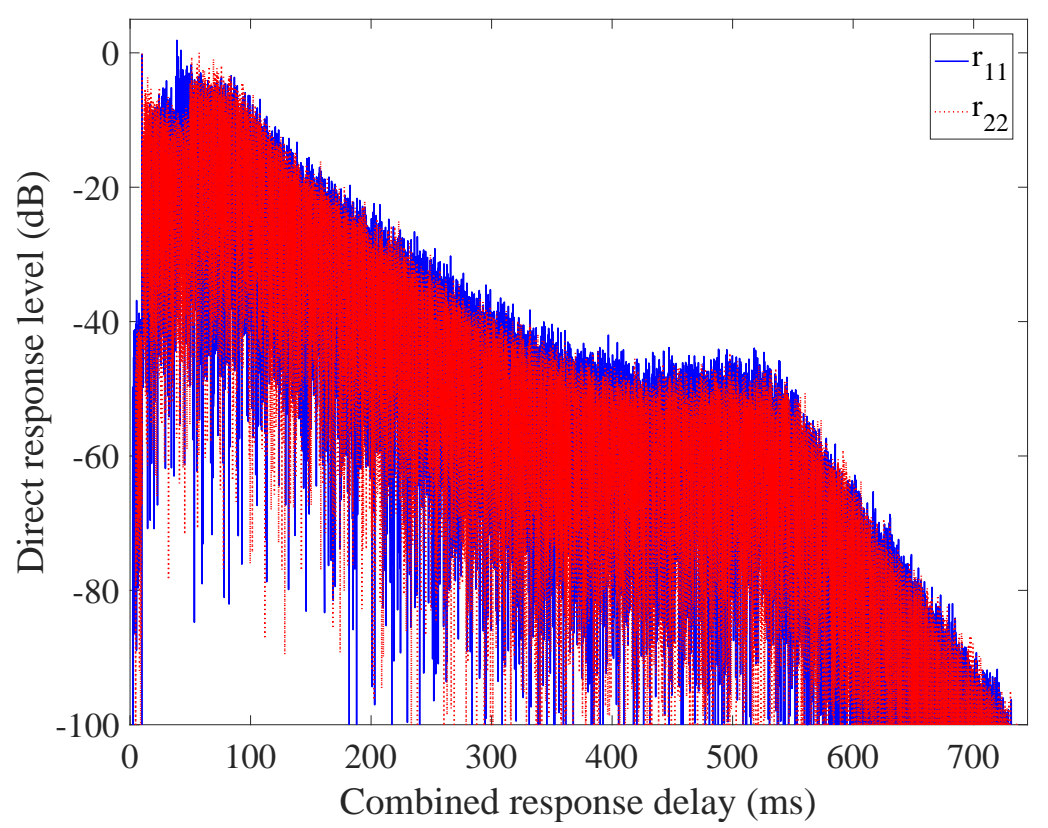

Figure 6.6: The two direct responses obtained with Algorithm C (Fast version of joint time and frequency shaping algorithm).

method than Algorithms A and B (Algorithm C) works by solving the time shaping algorithm using D- $\infty-1$ till half convergence (say, $N_{\text {iter }}=500$ ) and then alternating projection and DALM for few iterations, say five, to ensure that the frequency constraints are also satisfied. This algorithm makes use of the high computational speed of the DALM at the same time ensuring that the frequency constraints are satisfied. Since the projection onto each frequency constraint is done sequentially, some of the earlier constraints may be altered by a following projection. Therefore, we perform a few alternating steps of projection and DALM, say three, after the $500 \mathrm{D}-\infty-1$ steps to ensure that the frequency constraints are satisfied. It was found that this algorithm is around ten times faster than Algorithm B with $N_{\text {iter }}=100$ in addition to providing a CDR of $-39 \mathrm{~dB}$. The frequency constraining value $\gamma$ is chosen to be 85 th percentile value of the frequency response of the D- $\infty-1$ solution. It was found that an SFM of 0.854 was achieved in this case. The results obtained using this approach are shown in Figure 6.5, Figure 6.6 and Figure 6.7.

Comparing the results obtained with algorithms A, B and C from Table 6.1, it 


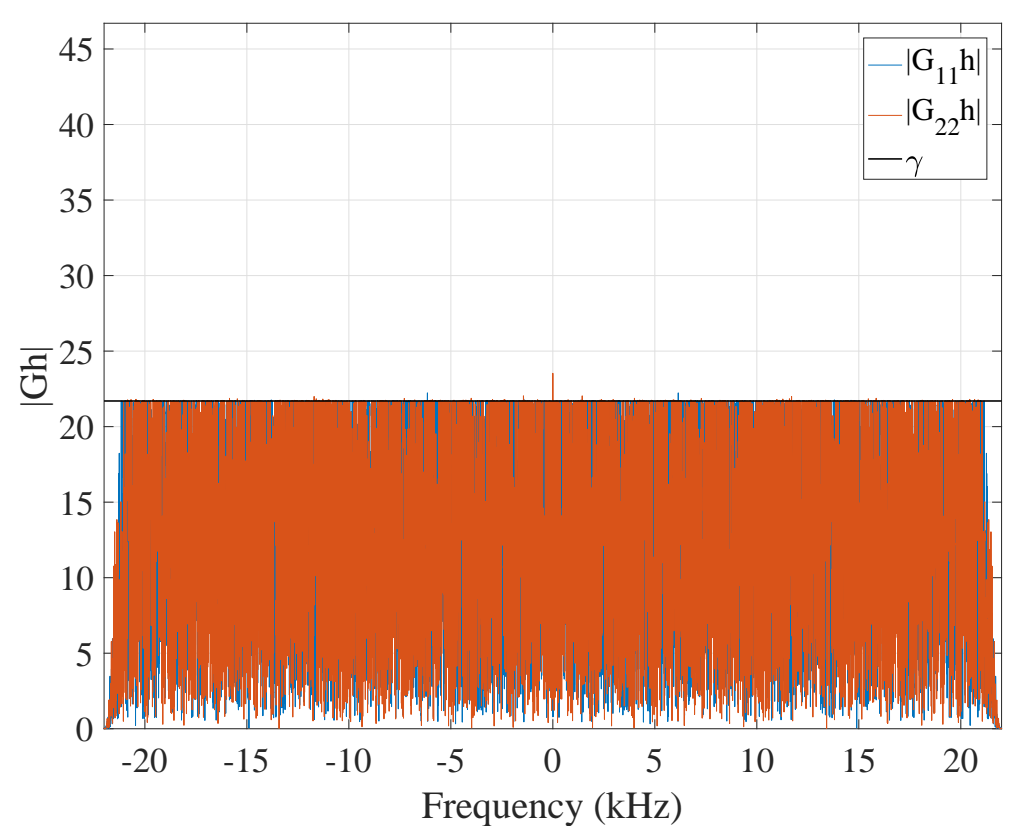

Figure 6.7: Frequency responses of the two channels obtained with Algorithm C (Fast version of joint time and frequency shaping algorithm).

can be seen that the best $\mathrm{nPRQ}$ value is obtained using algorithm $\mathrm{C}$ showing the best time domain shaping. In addition, good frequency flattening is achieved with algorithm C, as indicated by the SFM value. However, the cross-talk cancellation achieved using the algorithm is reduced compared to other algorithms, which is a trade off for the fast computational speed and good shaping.

The experimental studies were repeated with the other DALM formulations namely D-2-1, D-2-2 and D- $\infty-2$. Plots obtained were similar to those from D- $\infty$ 1. The results obtained with Algorithm $\mathrm{C}$ using a combination of these DALM formulations and projection are tabulated in Table 6.2. It can be seen that the CDR is marginally better for D-2-1 and D-2-2 when compared to D- $\infty-1$ and D$\infty-2$, but better spectral flatness is obtained with $\mathrm{D}-\infty-1$ and $\mathrm{D}-\infty-2$.

Comparing the results of the DALM formulations with and without frequency shaping in Table 6.2 , it can be seen that frequency shaping not only improves the frequency flatness, but also the time domain shaping performance. However, the cross-talk cancelation performance is affected which is the expected trade off for achieving good shaping performance. 


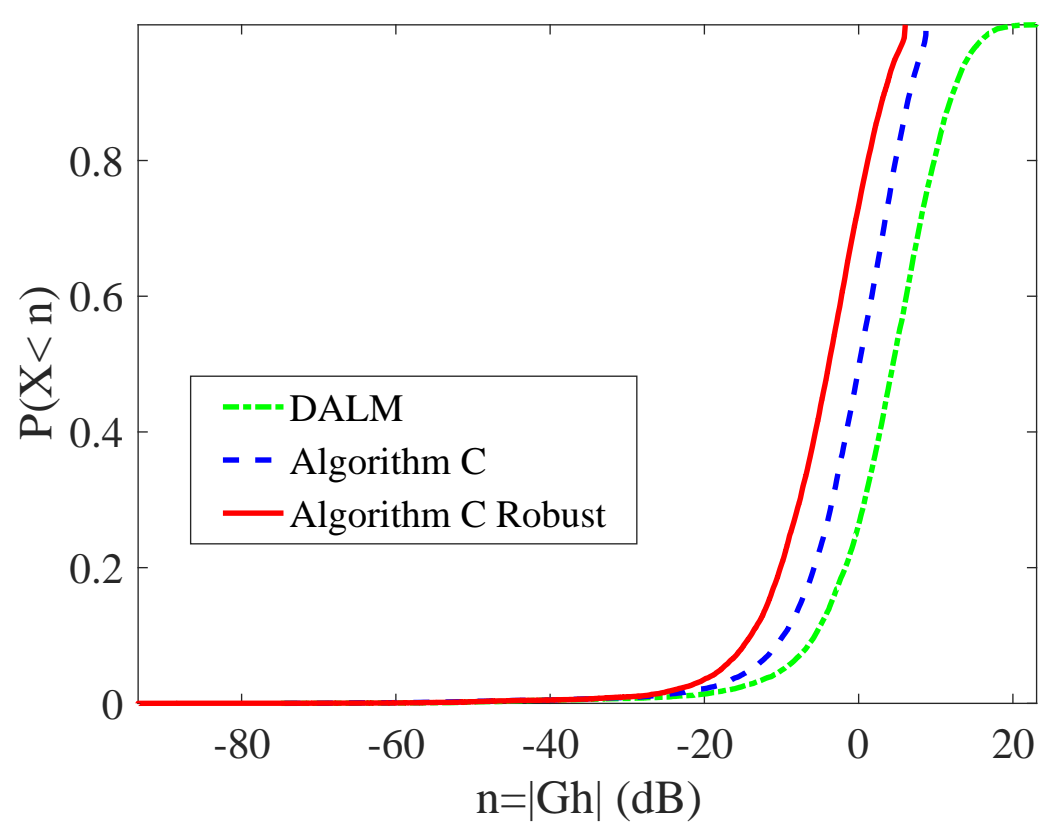

Figure 6.8: Cumulative density function of the frequency responses obtained with frequency unconstrained DALM, Algorithm C and spatially robust version of Algorithm C.

Algorithm $\mathrm{C}$ was thus found to provide reasonable performance in addition to higher computational speed, in spite of the approximations made to increase the computational speed. The next set of results were obtained when Algorithm C was run to solve the design approach over multiple microphone positions to achieve spatial robustness as explained in Section 6.3. 15 perturbed versions of the original channel for a microphone displacement of $1 \mathrm{~cm}$ were generated according to [39] using the same procedure as in Section 5.7 and Algorithm $\mathrm{C}$ was used to find shaping filters robust at the original and the perturbed positions. The results obtained are given in Table 6.3.

It can be seen that the $\mathrm{nPRQ}$ value improves with this robustness design compared to the original joint time and frequency shaping approach, though the CDR reduces. This is because there is a compromise between robustness and the performance; performance reduces slightly when robustness is incorporated but is similar at all the designed microphone positions. A comparison of the CDF of the frequency responses obtained using this robust version of Algorithm C, Algorithm 


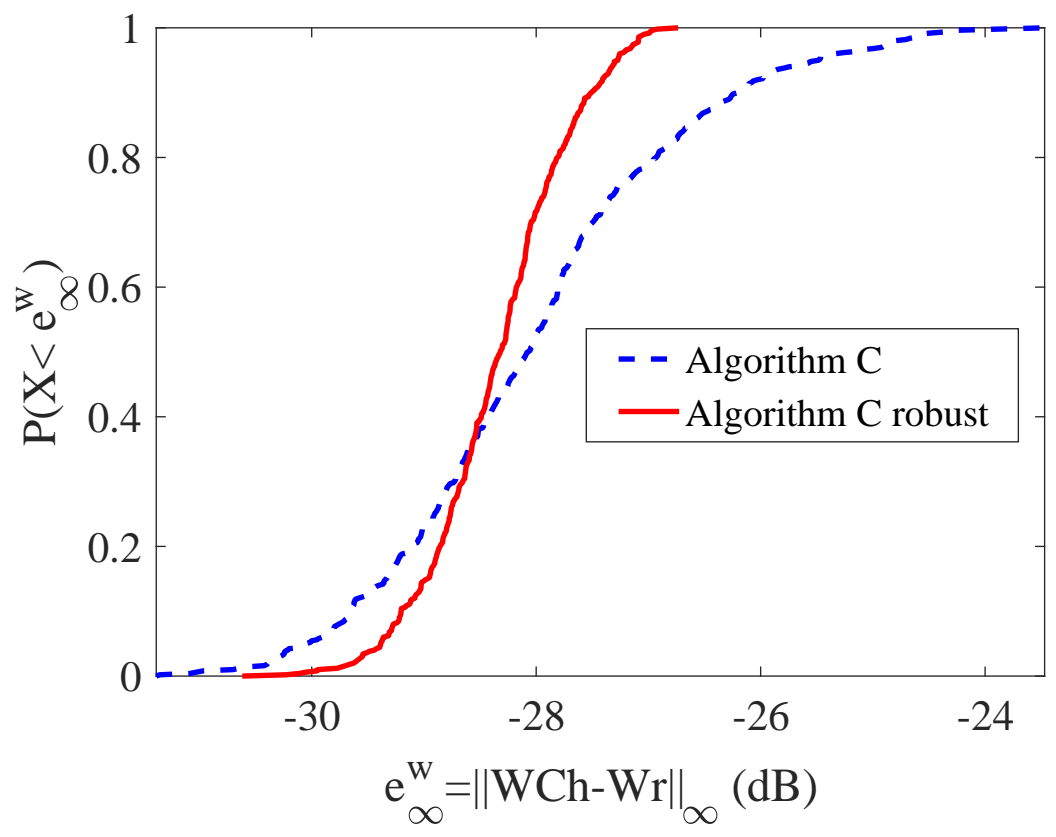

Figure 6.9: Cumulative density function of the CDR values obtained with Algorithm $\mathrm{C}$ and its spatially robust version.

$\mathrm{C}$ and DALM without explicit design for robustness are shown in Figure 6.8. As discussed earlier in Section 5.7, vertical axis of the CDF plot shows the probability of occurrence of values less than any value on the horizontal axis. Therefore, the steeper the slope, better the robustness. It can therefore be seen from Figure 6.8 that the robust version Algorithm $\mathrm{C}$ gives the best performance followed by Algorithm $\mathrm{C}$ and then DALM.

In order to compare the robustness of Algorithm $\mathrm{C}$ and its spatially robust version, 500 perturbed positions were generated at a distance of $1 \mathrm{~cm}$ from the original position as in Section 5.7 and the CDR values at these locations were calculated. The CDF of the CDR values obtained with Algorithm $\mathrm{C}$ and spatially robust Algorithm $\mathrm{C}$ with $\mathrm{D}-\infty-1$ are shown in Figure 6.9. It can be seen that the slope is higher for the robust approach when compared to the non-robust approach. The CDF plots for other DALM formulations are also similar. This indicates that the variation in CDR with perturbation in microphone position is less for the robust approach which validates the spatial robustness of the design. 
Table 6.2: Performance comparison of DALM formulations in shaping with and without frequency projection. Algorithm $\mathrm{C}$ is used for frequency projection. Performance metrics CDR, CPU time, SFM and nPRQ are defined as in Section 4.4. Objective function value is the final value obtained for the objective in (6.26) with $u$ and $v$ corresponding to the respective DALM formulation used.

\begin{tabular}{cccccc} 
DALM form & $\begin{array}{c}\text { CDR } \\
(\mathrm{dB})\end{array}$ & $\begin{array}{c}\text { CPU Time } \\
(\mathrm{s})\end{array}$ & $\begin{array}{c}\text { Objective } \\
\text { function }\end{array}$ & SFM & $\begin{array}{c}\text { nPRQ } \\
(\mathrm{dB})\end{array}$ \\
\hline D-2-1 with $N_{\text {iter }}=300$ & -41 & 118 & 0.3939 & 0.81 & 2.84 \\
\hline D-2-1 & -57 & 25 & 0.32 & 0.7459 & 3.94 \\
\hline D-2-2 with $N_{\text {iter }}=300$ & -42 & 126 & 0.417 & 0.792 & 3.64 \\
\hline D-2-2 & -57 & 28 & 0.309 & 0.723 & 4.73 \\
\hline D- $\infty-2$ with $N_{\text {iter }}=500$ & -38 & 372 & 0.781 & 0.847 & 3.86 \\
\hline D- $\infty-2$ & -58 & 88 & 0.536 & 0.79 & 4.39 \\
\hline
\end{tabular}

Table 6.3: Performance comparison of Algorithm $\mathrm{C}$ in the design over multiple microphone positions for various DALM formulations. Performance metrics CDR, CPU time, SFM and nPRQ are defined as in Section 4.4. Objective function value is the final value obtained for the objective in (6.26) with $u$ and $v$ corresponding to the respective DALM formulation used.

DALM form CDR (dB) CPU Time (s) Objective SFM nPRQ (dB) function

\begin{tabular}{cccccc}
\hline D-2-1 & -34 & 247 & 0.587 & 0.829 & 2.17 \\
\hline D-2-2 & -31 & 262 & 0.613 & 0.821 & 3.31 \\
\hline D- $\infty-1$ & -28 & 1412 & 0.818 & 0.832 & 3.72 \\
\hline D- $\infty-2$ & -28 & 1426 & 0.82 & 0.824 & 3.68 \\
\hline
\end{tabular}

\subsection{Contributions from this chapter}

The main contribution of this chapter is the development of an efficient projection method onto a low dimensional norm ball and its application to impulse response 
shaping to impose frequency domain constraints. The projection step is used as a proximal mapping in conjunction with the fast DALM based impulse response shaping algorithm discussed in earlier chapters. The projection step ensures that frequency response flattening is obtained in addition to the desirable time domain characteristics obtained using the DALM based shaping approach. The algorithm works by alternating the DALM iterations and the projection step one after the other. The experimental results demonstrate that the algorithm works well for the shaping problem, providing frequency response flattening in addition to a good cross-talk cancelation and the desired shaping in the time domain. A computationally faster variant of this algorithm performs a small number of alternating projection and DALM steps after several DALM iterations. It has been found from the experimental results that the new algorithm is around 1000 times faster than the original algorithm.

This chapter also explains the extension of the projection approach to a design over multiple microphone positions to achieve spatial robustness. Thus, the projection method offer advantages for the fast DALM based shaping approach to achieve joint time and frequency domain shaping, without compromising on the computational speed and performance. The projection approach has applicability to a wide range of convex problems due to small memory requirement and high computational speed, although we proposed it here for the specific application of acoustic impulse response shaping. 


\section{Chapter 7}

\section{Statistically Robust Shaping Formulation}

\subsection{Background}

Joint shaping and cross-talk cancelation formulations that solve for the shaping filters based on measured room responses were discussed in the earlier chapters. Measurement errors or variations in room response (due to environmental conditions and microphone positioning) can arise, resulting in a poor filter design. Robustness of filters to at least some of these variations is an important design consideration. A design approach over multiple microphone positions offering robustness to microphone position error is presented in $[28,39,121]$, although its implementation is computationally expensive. A computationally faster model of this work is discussed in Chapter 5 based on the DALM formulations developed. These models are based on generating synthetically-generated perturbed channels using a detailed theoretical analysis of the errors caused by small changes in microphone positions in [38].

A more appropriate approach for achieving robustness is to statistically constrain the channel variations. One such approach [57,81-83] aims to design shaping filters adaptively by probabilistically modelling channel variability. This is achieved using a feed-forward scheme of filter design that uses some initial assumptions based on properties of room impulse response. The approach presented in this chapter aims to probabilistically constrain the error between the desired and resultant responses to be less than a threshold. The approach applied is some- 
what similar to [39], but rather than generate perturbed copies of the channel, the perturbation model is included into the formulation of the design problem. This is achieved by explicitly constraining (to a small number) the probability of large error in the combined responses. This optimization problem involves a non-convex probability constraint which has been slightly simplified using some properties of the room response to approximate the problem by a convex one and make it mathematically tractable. These simplifications are similar to those employed in [81]. The evaluation of the results are performed on a more detailed model presented in [39] that empirically justifies the modelling simplifications made. The details of the model and evaluations are described in the sections to follow. The contents of this chapter are based on the paper "A statistically robust approach to Acoustic Impulse Response Shaping" [122].

\subsection{Problem Definition}

The joint shaping and cross-talk cancelation problem is solved by a regularized weighted error minimization as in (4.8) in Chapter 4 given by

$$
\min _{h}\|W C h-W r\|_{u}+\lambda\|h\|_{v}
$$

where $u$ and $v$ are the norms, $C$ is the block channel Toeplitz matrix, $W$ is the diagonal weight matrix, $r$ is the expected response and $\lambda$ is the regularization parameter. Vector $r$ is set to be one at the position of the direct path in the direct responses and zero at all other sample points. The formulation proposed in this chapter uses the same notation, but a different approach involving the use of probability constraints to achieve the desired response.

A change in microphone position causes the channel vector $c$ to be perturbed $[39,111]$ as

$$
d_{m l}=c_{m l}+q_{m l}
$$

where $q$ is a vector of perturbations. Now consider matrix $D$ consisting of Toeplitz convolution matrices from the perturbed impulse responses $d_{m l}$ in a manner analogous to that of matrix $C$. A statistically robust formulation for shaping aims to ensure that even if $d \neq c$ due to channel perturbations, the probability of $|D h-r|$ being large is constrained where $r$ is a delta function with a peak at the location of the direct path. This is the type of robustness approach used in [40]. Thus, the 
formulation proposed in this work constrains the probability of the error $\left|d_{k}^{\mathrm{T}} h-r_{k}\right|$ being greater than a threshold $\gamma$ to be less than a pre-defined probability value for each $d_{k}^{\mathrm{T}} h$, where $d_{k}^{\mathrm{T}}$ is the $k$ th row of matrix $D$. Therefore, the proposed optimization problem to be solved for finding the vector of shaping filters $(h)$ is given by

$$
\begin{aligned}
& \min _{\gamma, h} \gamma \\
& \text { subject to } \operatorname{Pr}\left(\left|d_{k}^{\mathrm{T}} h-r_{k}\right|>\gamma\right) \leq \mathrm{P}_{\max , k} \quad k \in\left[1, \ldots, M^{2} N_{\mathrm{r}}\right]
\end{aligned}
$$

where $\operatorname{Pr}$ denotes the probability, $\mathrm{P}_{\max }$ is the maximum allowed probability that the error $\left|d_{k}^{\mathrm{T}} h-r_{k}\right|$ is greater than threshold $\left.\gamma . k \in\left[1, \ldots, M^{2} N_{\mathrm{r}}\right]\right)$ because constraints are defined for all sample points in $r$. (Length of $r$ is $M^{2} N_{\mathrm{r}}$ as in Section 4.1.) $\mathrm{P}_{\max , k}$ being defined separately for each value of $k$ allows a 'don't care' region in the GIR, but heavy penalisation of pre-echo, late reverberation and cross-talk. $\mathrm{P}_{\max , k}$ is chosen to be very small for the cross-talk $(m \neq s)$ and late reverberation and pre-echo in the direct channels $(m=s)$, but is higher for the early reflections. This indirectly imposes a similar effect to the weighting used in $[21,27]$ where the weighted error $\|W(C h-r)\|$ is minimized.

The formulation in (7.2) gives a statistically robust solution for shaping filters, but the problem is non-convex. The main task is, therefore, to convert this problem into a convex problem to find a solution using standard algorithms.

\subsubsection{Conversion to a convex problem}

In order to make the optimization problem more specific and convert the constraint in (7.2) to a convex constraint, the first step is to find the distribution of the perturbation vector $q$. It can be shown using an approach based on [123] that for small sensor position perturbation, or strong multipath, the sensor position errors are Gaussian. Thus, $q_{k}$ is well represented by a Gaussian distribution $q_{k} \sim \mathcal{N}\left(0, \iota^{2}\right)$. A justification for this assumption is detailed in Appendix B. The variance of the perturbations $\iota^{2}$ for a particular shift in microphone position at a particular temperature in a diffuse field can be found using (2.17) [38].

The problem is further simplified by modelling all the components of $q$ as independent and identically distributed (IID). An empirical justification of this approximation using an IID Gaussian model is presented in Section 7.3. 
From this IID approximation, it follows that $d_{k}^{\mathrm{T}} h-r_{k}$ also has a Gaussian distribution with mean $\mu_{k}=c_{k}^{\mathrm{T}} h-r_{k}$ and variance given by

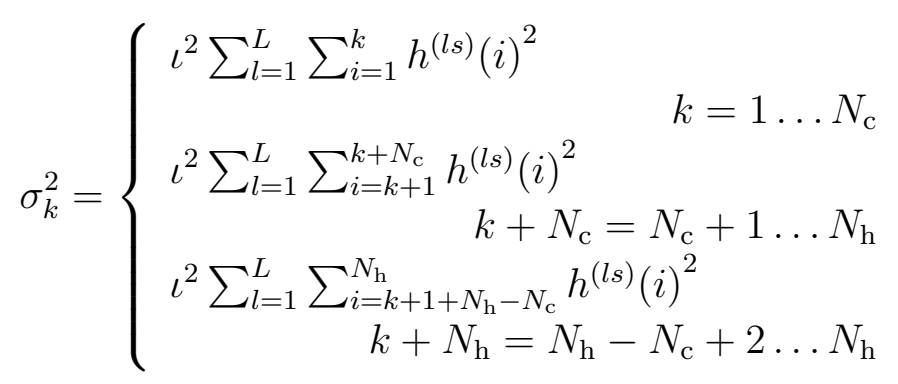

where $N_{\mathrm{c}}$ is the length of each channel $c_{m l}$ and $N_{\mathrm{h}}$ is the length of each filter $h_{l s}$ same as in Section 4.1. This expression is computed based on the structure of matrix $C$ as an overcomplete dictionary (as in (4.4) for obtaining a sparse solution for $h$. The sparse solution ensures robustness to measurement errors as can be seen from the results in Chapter 5. Calculation of this variance for a small channel length is shown in Appendix B.

Since the probability constraint is based on $|D h-r|$, we have to find the probability density function (PDF) and hence the cumulative density function (CDF) of each element of $|D h-r|$ given that each element of the vector $D h-r$ has a Gaussian distribution. In order to find the PDF, let $x=d_{k}^{T} h-r_{k}$ and $y=|x|$. The solutions of the equation $y=|x|$ are $x= \pm y$. To derive the PDF $p_{y}(y)$ of a function of the random variable, we use the approach of Papoulis et al. [124], using which $p_{y}(y)$ can be written as

$$
\begin{aligned}
p_{y}(y) & =p_{x}(y) /|1|+p_{x}(-y) /|-1| \\
& =\frac{1}{\sqrt{2 \pi \sigma_{k}^{2}}}\left(\mathrm{e}^{-\left(y-\mu_{k}\right)^{2} / 2 \sigma_{k}^{2}}+\mathrm{e}^{-\left(y+\mu_{k}\right)^{2} / 2 \sigma_{k}^{2}}\right)
\end{aligned}
$$

and hence the $\mathrm{CDF} F_{y}(y)$ is given by

$$
\int_{0}^{y} p_{y}(y) d y=\frac{1}{\sqrt{2 \pi \sigma_{k}^{2}}} \int_{0}^{y} \mathrm{e}^{\frac{-\left(y-\mu_{k}\right)^{2}}{2 \sigma_{k}^{2}}}+\mathrm{e}^{\frac{-\left(y+\mu_{k}\right)^{2}}{2 \sigma_{k}^{2}}} d y
$$

This is known as the folded normal distribution [125].

Let $u=\left(y-\mu_{k}\right) / \sqrt{2} \sigma_{k}$ in the first term and $v=\left(y+\mu_{k}\right) / \sqrt{2} \sigma_{k}$ in the second 
term. Hence, $F_{y}(y)$ is given as

$$
\begin{aligned}
& F_{y}(y)= \frac{\int_{0}^{\left(y-\mu_{k}\right) / \sqrt{2} \sigma_{k}} \mathrm{e}^{-u^{2}} d u \sqrt{2} \sigma_{k}}{\sqrt{2 \pi \sigma_{k}^{2}}} \\
&+\frac{\int_{0}^{\left(y+\mu_{k}\right) / \sqrt{2} \sigma_{k}} \mathrm{e}^{-v^{2}} d v \sqrt{2} \sigma_{k}}{\sqrt{2 \pi \sigma_{k}^{2}}} \\
&=\frac{1}{2}\left(\operatorname{erf}\left(\frac{y-\mu_{k}}{\sqrt{2} \sigma_{k}}\right)+\operatorname{erf}\left(\frac{y+\mu_{k}}{\sqrt{2} \sigma_{k}}\right)\right)
\end{aligned}
$$

where $\operatorname{erf}()$ is the error function. Using (7.6), the constraint in the proposed optimization problem (7.2) for $\mathrm{P}(y>\gamma)=1-\mathrm{P}(y \leq \gamma) \leq \mathrm{P}_{\max }$ can now be written as

$$
\frac{1}{2}\left(\operatorname{erf}\left(\frac{\gamma-\mu_{k}}{\sqrt{2} \sigma_{k}}\right)+\operatorname{erf}\left(\frac{\gamma+\mu_{k}}{\sqrt{2} \sigma_{k}}\right)\right) \geq 1-\mathrm{P}_{\max }
$$

This constraint is non-convex in parameter $\gamma$ due to the presence of the pair of error functions. We can approximate this constraint by a convex one, by defining the constraint separately for different regions of the direct response and the crosstalk. For the direct path and early reflections, $\mu$ usually deviates from zero by significantly more than $\sigma_{k}$. Therefore, we approximate the constraint by

$$
\frac{1}{2} \operatorname{erf}\left(\frac{\gamma-\mu_{k}}{\sqrt{2} \sigma_{k}}\right) \geq 1-\mathrm{P}_{\text {max }_{\text {early }}}
$$

although this approximation is conservative i.e., it overestimates the probability that the constraint is violated, when $\mu_{k}$ is negative. Equivalently

$$
\gamma+\left(c_{k}^{\mathrm{T}} h-r_{k}\right) \geq \sqrt{2} \sigma_{k} \operatorname{erfi}\left(2\left(1-\mathrm{P}_{0, \max } / w_{\text {early }}\right)\right)
$$

where erfi() is the inverse error function, $\mathrm{P}_{0, \max }$ is a fixed probability value and $w_{\text {early }}$ is a weight vector penalising early reflections.

In the case of late reverberation, $\mu_{k} \approx 0$ results in both the error functions being approximately the same. The constraint in this case can thus be written as

$$
2 \times \frac{1}{2} \operatorname{erf}\left(\frac{\gamma-\mu_{k}}{\sqrt{2} \sigma_{k}}\right) \geq 1-\mathrm{P}_{\max _{\text {late }}}
$$

or

$$
\gamma+\left(c_{k}^{\mathrm{T}} h-r_{k}\right) \geq \sqrt{2} \sigma_{k} \operatorname{erfi}\left(1-\mathrm{P}_{0, \max } / w_{\text {late }}\right)
$$




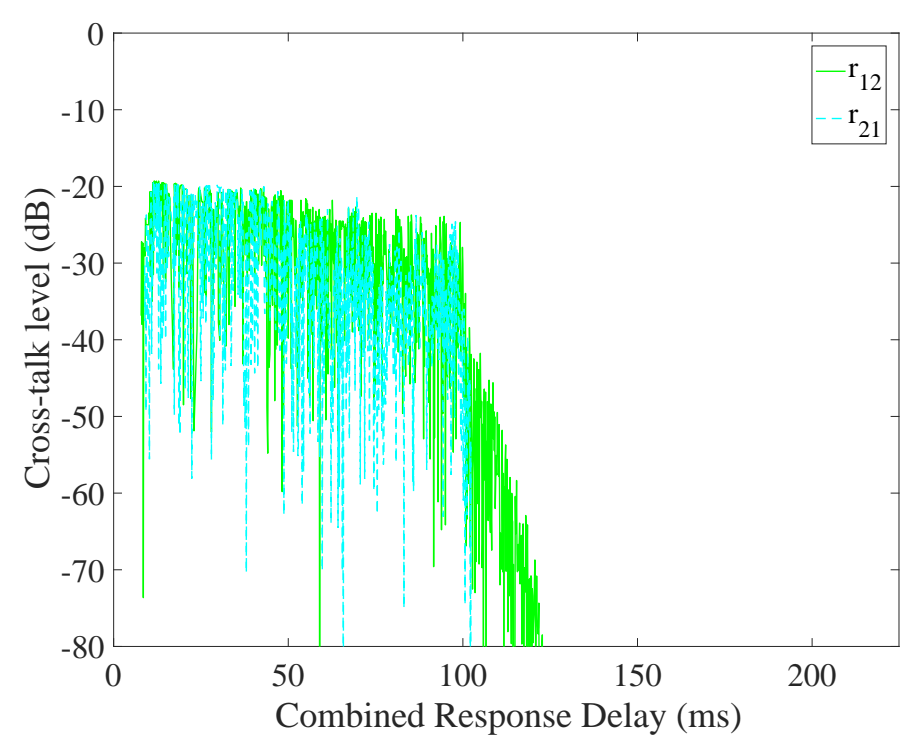

Figure 7.1: Cross-talk responses $r_{12}$ and $r_{21}$.

The pre-echo in the direct response and cross-talk also have $\mu_{k} \approx 0$ resulting in the constraint being similar to that of the late reverberation. Thus, the final optimization problem can be written as

$$
\min _{\gamma, \mathbf{h}} \gamma
$$

subject to $\mathbf{B h}=\mathbf{1}$

$$
\begin{array}{ll}
\gamma+\left(\mathbf{c}_{k}^{\mathrm{T}} \mathbf{h}-r_{k}\right) \geq \sqrt{2} \sigma_{k} \operatorname{erfi}\left(2-2 \mathrm{P}_{0, \max } / w_{\text {early }}\right), & k \in \mathbb{A}_{3} \\
\gamma+\left(\mathbf{c}_{k}^{\mathrm{T}} \mathbf{h}-r_{k}\right) \geq \sqrt{2} \sigma_{k} \operatorname{erfi}\left(1-\mathrm{P}_{0, \max } / w_{\text {late }}\right), & k \in \mathbb{A}_{4} \\
\gamma+\left(\mathbf{c}_{k}^{\mathrm{T}} \mathbf{h}-r_{k}\right) \geq \sqrt{2} \sigma_{k} \operatorname{erfi}\left(1-\mathrm{P}_{0, \max } / w_{\text {preecho }}\right), & k \in \mathbb{A}_{1} \\
\gamma+\left(\mathbf{c}_{k}^{\mathrm{T}} \mathbf{h}-r_{k}\right) \geq \sqrt{2} \sigma_{k} \operatorname{erfi}\left(1-\mathrm{P}_{0, \max } / w_{\text {crosstalk }}\right), & k \in \mathbb{A}_{5}
\end{array}
$$

where $\mathbf{1}$ is a $M \times 1$ vector of $1 \mathrm{~s}$, and the matrix $\mathbf{B}$ consists of the $M$ rows of the matrix $\mathbf{C}$ corresponding to the direct paths of the direct responses. This constraint ensures that the value of the direct response just before the 'don't care' region is $1[27]$ to avoid the trivial solution of $h=0$. Region $\mathbb{A}_{1}$ is the pre-echo, $\mathbb{A}_{3}$ are the early reflections and $\mathbb{A}_{4}$ are the late reverberations respectively of the direct 


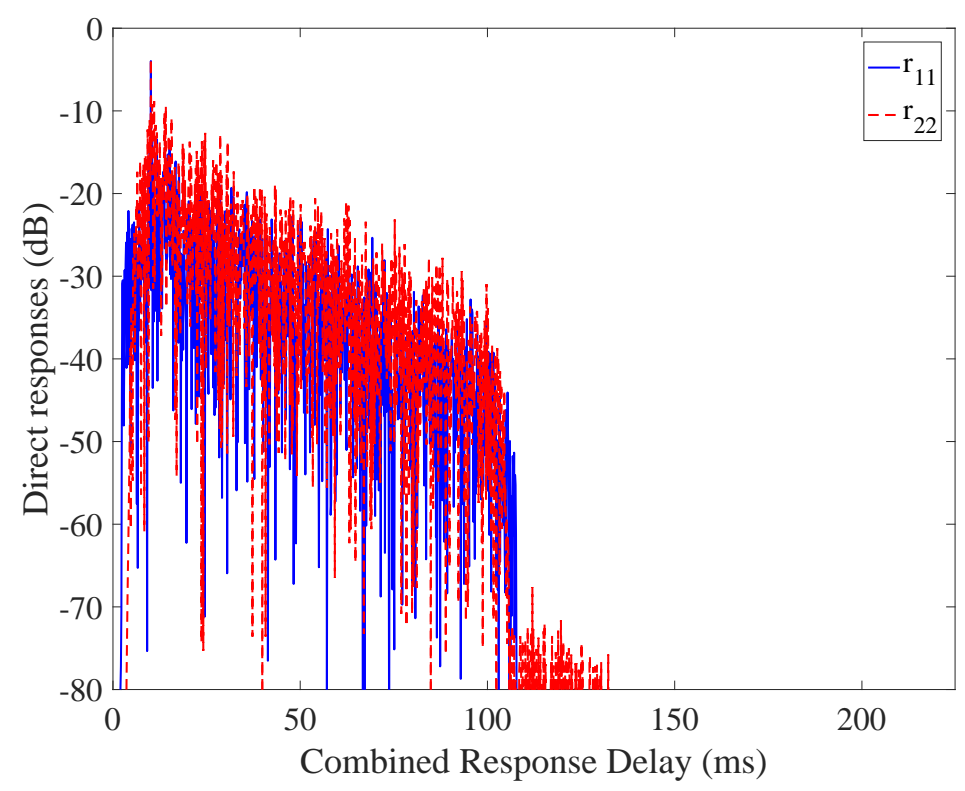

Figure 7.2: Shaped direct responses $r_{11}$ and $r_{22}$.

responses as in (4.6). The region $\mathbb{A}_{5}=\left[1, N_{\mathrm{r}}\right]$ is the cross-talk. (There are no desired portions in the cross-talk responses.) This optimization problem can be expressed in the form of a second order conic program (SOCP), which is convex. The expression of (7.13) in a standard SOCP form is detailed in Appendix B.

\subsection{Simulation Results}

The statistically robust problem in (7.13) was solved for channels measured at $8 \mathrm{kHz}$ sampling rate in the same room and same setup as in Figure 5.1 in Section 5.7 having $T_{60}=245 \mathrm{~ms}$, with $L=3, M=2, N_{\mathrm{c}}=800, N_{\mathrm{r}}=1799$ using the convex solver CVX [126]. The lengths of the pre-echo, early reflection and late reverberation regions in the direct response were set to be $10 \mathrm{~ms}, 40 \mathrm{~ms}$ and $175 \mathrm{~ms}$ [27] as in Section 5.7. $P_{\max }$ was set to be 0.8 for the 'don't-care' regions and 0.2 for pre-echo and cross-talk. The variance of perturbations [38] $\left(\iota^{2}=0.17\right.$ times the power of the impulse responses $c$ [39, eq.(9)]) was chosen to be equivalent to $1 \mathrm{~cm}$ shifts in microphone position at $4 \mathrm{kHz}$ and at $20^{\circ} \mathrm{C}$ in a diffuse field.

The performance metric used in this work is CDR. In order to evaluate the robustness of the filter designs, 500 perturbed versions of the original channel 


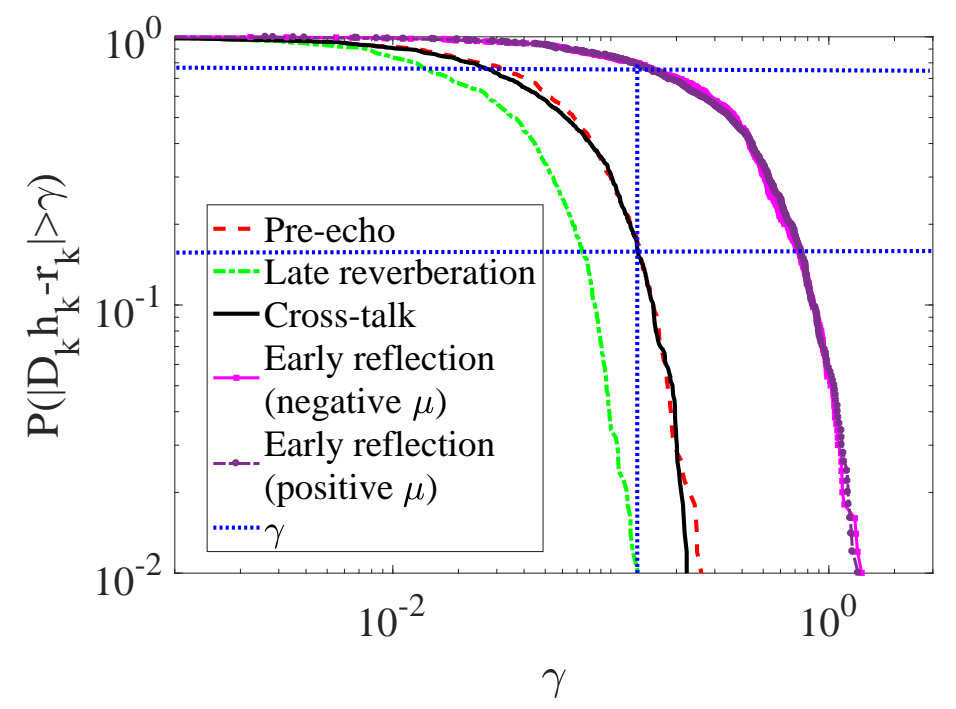

Figure 7.3: Distribution of typical points within the pre-echo, late reverberation, cross-talk and early reflection regions over simulated perturbed channels using the proposed approach. The vertical dotted line indicates the optimized value of $\gamma$. The intersections of the dotted lines indicates the target: the probability of deviation from the desired response $\left|D_{k} h_{k}-r_{k}\right|$ being greater than $\gamma$ is less than $P_{\max }$ as in (7.13).

were generated using the diffuse field perturbation model defined in [39] as in Section 5.7. Note that this model of perturbations is more realistic than the IID Gaussian assumption used in deriving (7.3), and does not involve the approximations involved in deriving the convex expressions (7.10) and (7.12), and hence tests the validity of the formulation in a realistic scenario.

Typical cross-talk and shaped responses are shown in Figure 7.1 and Figure 7.2 respectively. The shaped responses in Figure 7.2 show the peak is around $19 \mathrm{~dB}$ above the smaller values and the response decays rapidly after the allowed early reflections. As intended, the design process maintains the peak in the shaped response and some of the early reflections whilst reducing late reverberation. Figure 7.1 shows that cross-talk cancelation of around $-20 \mathrm{~dB}$ has been achieved. The computational time was found to be $958 \mathrm{~s}$ for this problem.

The distribution of the performance over the perturbed channels are shown in Figure 7.3 to study the efficacy of the robust implementation. The solid curve 


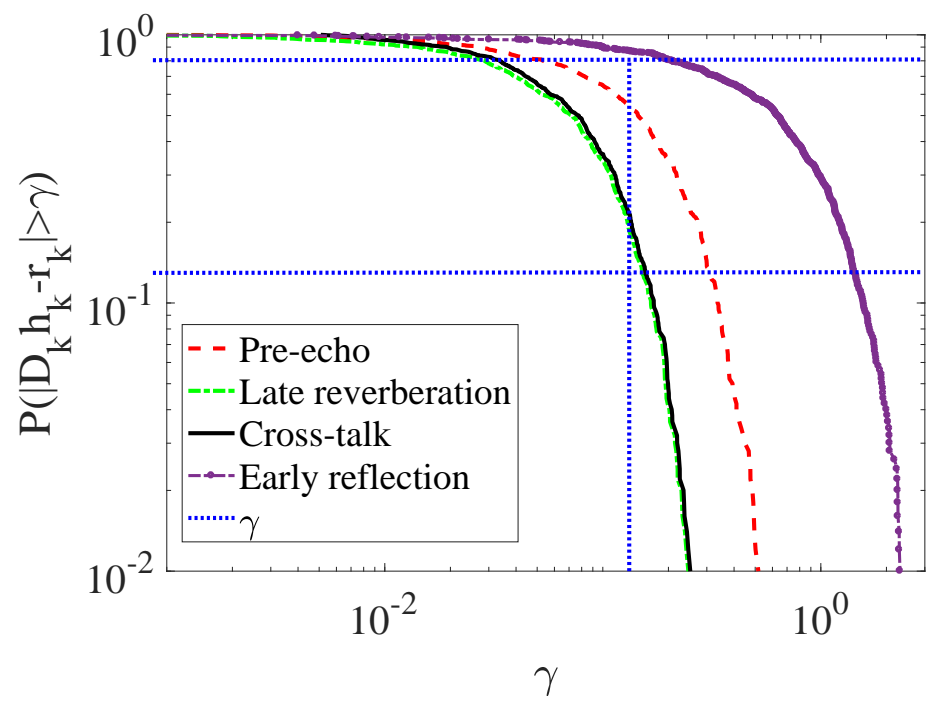

Figure 7.4: Distribution of typical points within the pre-echo, late reverberation, cross-talk and early reflection regions over simulated perturbed channels without explicit consideration of robustness. Here the requirement that the probability of deviation greater than $\gamma$ being less than $P_{\max }$ is not met.

corresponds to the behaviour at a representative sample point in the cross-talk. Here, the probability of the deviation from the desired response being greater than the threshold $\gamma, \mathrm{P}\left(\left|D_{k} h_{k}-r_{k}\right|>\gamma\right)$, obtained is $\leq 0.2$, which is the designed $\mathrm{P}_{\max }$ value. Therefore, the probability constraint in (7.8) is satisfied. Similarly, the dotdashed and dashed curves correspond to representative sample points of the late reverberation and the pre-echo region of the direct response respectively. Again, it can be seen that the constraint is satisfied demonstrating the robustness of the formulation. Two points in the early reflection region of the direct responses are shown, one with a positive $\mu$ and other with a negative $\mu$ before perturbation to verify the approximation in (7.9) in which one $\operatorname{erf}()$ function is ignored. From Figure 7.3, the probability $\mathrm{P}\left(\left|D_{k} h_{k}-r_{k}\right|>\gamma\right)$ in both cases is less than or equal to 0.8 , which is the designed $\mathrm{P}_{\max }$ value.

A similar analysis of the shaping performance is conducted for our previous non-robust shaping approach [110] (using the solution algorithm DALM) in which we do not explicitly design for robustness. It can be observed from the plot in Figure 7.4 that the probability of large deviations from the desired response is larger, and does not satisfy the constraint. This is especially true for points in the 


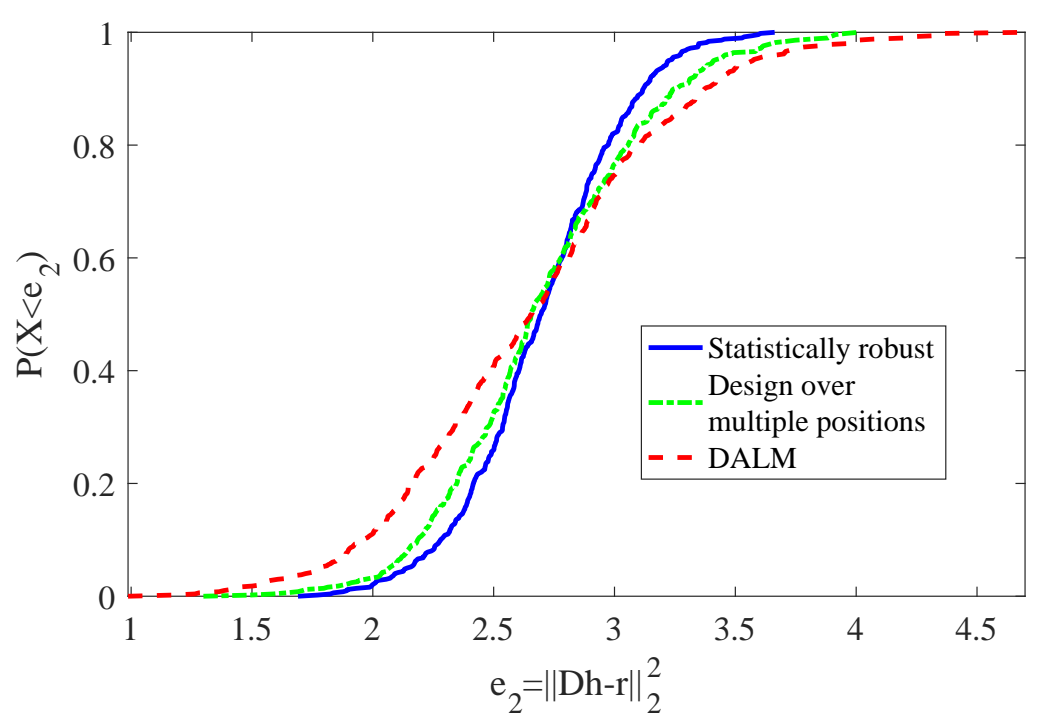

Figure 7.5: Cumulative density functions of the $\ell_{2}$ error on a normal scale.

response close to the 'don't care' regions.

The statistically robust approach is compared with the empirically robust approach [39] of design over multiple microphone positions. 14 perturbed positions at a distance of $1 \mathrm{~cm}$ from the original position were chosen. The empirical CDF of the $\ell_{2}$ norm of the deviation from the desired response shape, $e_{2}=\|D h-r\|_{2}^{2}$ is presented for the proposed approach, the DALM approach, and the empirical robust approach in Figure 7.5. It can be seen that the CDF slope is highest for the proposed approach, lower for the empirical approach and least for the DALM approach. As discussed earlier in Section 5.7 and Section 6.4, a steeper slope indicates better robustness demonstrating that the proposed approach is more robust to channel perturbations than the design over multiple positions. Thus the probability of large deviations is reduced, but at the cost of larger probability of small deviations. The plot for $\ell_{\infty}$ performance metric, $e_{\infty}=\|D h-r\|_{\infty}$ (not shown) is similar highlighting the robustness of the proposed approach over the non-robust approach.

The CDF of the maximum cross-talk value for the three approaches is shown in Figure 7.6. It can be seen that, as expected, the slope is lowest for the DALM approach, higher for the empirical approach, and highest for the proposed approach: offering the highest probability that large errors are small, but again, at the cost 


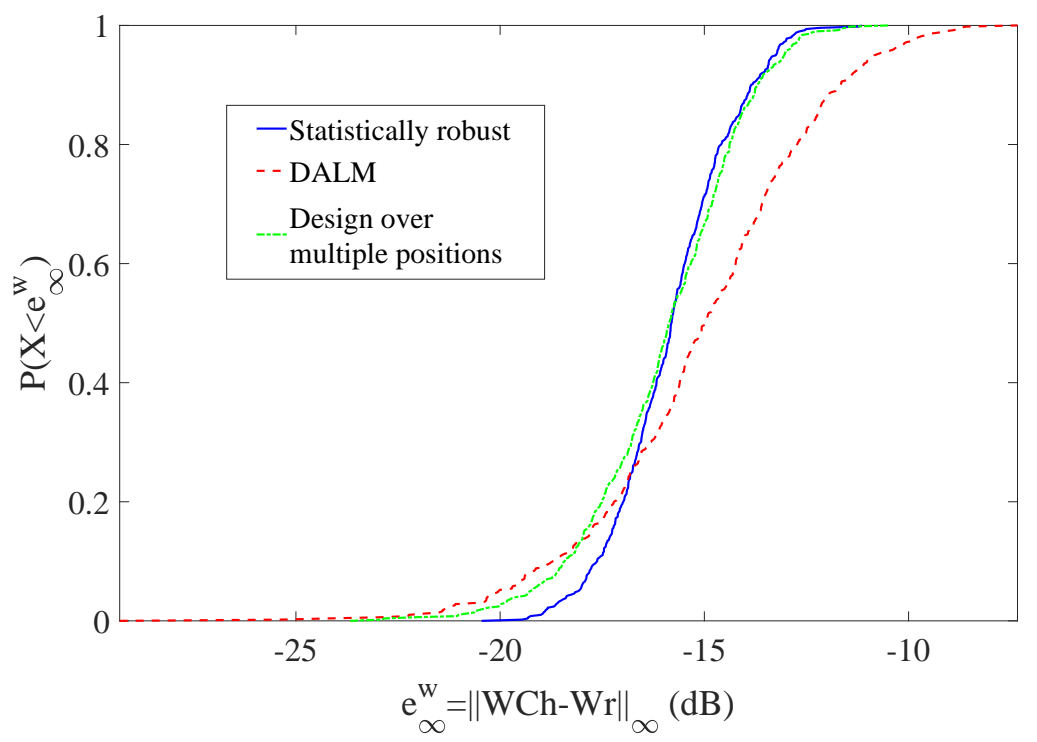

Figure 7.6: Cumulative density functions of the $\ell_{\infty}$ weighted error, which gives the maximum (worst) cross-talk level.

of larger probability of small errors.

\subsection{Contributions from this chapter}

A main contribution from this chapter is the development of a statistically robust approach for joint shaping and cross-talk cancelation of the room. The non-convex optimization formulation involving a probability constraint was approximated by a convex SOCP problem, and the validity of the approximation was confirmed by evaluation of the solution.

It has been demonstrated based on the results in Section 7.3 that the formulation is robust; obeying the constraint that the probability of large errors must be small. This formulation also provides robustness to channel variations caused by other factors like temperature. At present, this analytical approach results in slower computation than an equivalent empirical approach based on a design over multiple microphone positions. But this may change shortly due to recent developments in efficient algorithms for solving some SOCPs [127]. Once such an algorithm is devised, this approach can be very useful for providing robustness in real scenarios. This opens a scope for future work. 
A more accurate model would describe frequency dependence in the channel variances, especially at low frequencies. This opens a scope for future work. 


\section{Chapter 8}

\section{Pressure Matching Robust to Temperature Change}

\subsection{Background}

Sound field reproduction is an active research area which was discussed briefly in Chapter 2. The aim in the sound field reproduction problem is to create an intended sound field around the listener. One approach is the active compensated sound field reproduction (AC-SFR) $[128,129]$ in which the loudspeakers are pre-calibrated to achieve the desired sound field reproduction. A typical sound reproduction environment such as a room introduces reverberation, which may be either exploited or compensated for. The AC-SFR approach presented in this chapter uses the pre-calibration of a loudspeaker system. This approach has advantages over adaptive filter approaches $[16,130-135]$ in that the loudspeakers must be continually adapted and an in-situ microphone array needs to be constantly in place.

The approach for performing AC-SFR using pre-calibration of a loudspeaker system is presented in this chapter. A pre-calibrated solution has an advantage of not requiring a microphone to be located in-situ. However, unlike an adaptive solution, the pre-calibrated approach cannot update to compensate for changes in environmental conditions and hence the filter design must be inherently robust. Robustness is a problem to this approach, due to the perturbation of RIRs induced by environmental factors such as temperature or humidity changes, or changes in the room geometry. A robust filter design could be a good option to overcome this 
issue.

One promising way of achieving a robust design is by using impulse response shaping (IRS), which reduces the stringency of requirements for active compensation. IRS solutions have already been applied for equalizing loudspeaker-microphone channels and more efficient (MIMO) cross-talk cancelation systems [21, 121] to provide binaural listening for multiple listeners [58]. However, these IRS approaches have not been applied to sound field reproduction.

In AC-SFR, early reflections may be used to help construct the reproduced sound field. Solutions have been proposed using a ring of loudspeakers [128]. However, using a small number of higher order loudspeakers capable of directing the sound around the room [136-139], the loudspeakers can be made to exploit first and higher order reflections to create a virtual source $[129,140]$. It is then possible to produce a virtual source from a direction at which there is no loudspeaker driver, by for example directing sound energy towards the walls. However, such methods require pre-exciting the room. To actively compensate for the room reverberation, it is required to pre-excite the room, the required filters may be extremely lengthly and possess significant anti-causal components. Methods have been designed to reduce or eliminate the anti-causal components [141] but the perceptual effects on spatial image quality seem unclear.

The fine structure of RIRs is influenced not only by spatial position and changes in the room geometry but also the speed of sound. A change in the speed of sound affects the time taken for a wave front to propagate a certain distance. The change in propagation times results in a dilation of the RIR. The speed of sound is, in turn, influenced by changes in temperature and humidity. This RIR perturbation causes errors in any room equalization system, including AC-SFR systems. This ultimately results in pre-echo or pre-reverberation [142] in the resultant impulse responses delivered to the listener. A change in the average room temperature may be compensated by digitally dilating pre-measured RIRs using thermometermeasured temperature changes $[143,144]$.

In this chapter, a long propagation distance is shown to be more heavily influenced by temperature than a short one. This fact is used to show that higher order wall reflections are more prone to perturbation from temperature changes than lower order reflections. A penalty is therefore imposed on the anti-causal 
components of designed loudspeaker filters, so as to reduce the error caused by a change in temperature, and thereby obtain a more temperature-robust AC-SFR design. The DALM method introduced in earlier chapters is then applied to numerically solve the resulting optimization problem.

Our goal is to perform an AC-SFR design that reduces the temperature susceptibility to anti-causal components. A simple approach is to perform a minimum phase equalization. This approach however obliterates the phase response, which may destroy the fidelity of the acoustic image. A more powerful approach is the robust quadratic control approach of Brannmark et al. [56, 57, 81]. Simpler designs can also be performed by optimizing the planarity [145], although planarity control is not a topic of investigation of this paper.

\subsection{Temperature robustness}

A perturbation in room temperature causes a variation in the speed of sound. Consider an RIR between a loudspeaker and a microphone in a room. This can be well-modeled with the finite impulse response $c(t)$ of $N_{\mathrm{x}}$ propagation paths resulting from wall reflection given by

$$
c(t)=\sum_{n=0}^{N_{\mathrm{x}}} a_{n} \delta\left(t-d_{n} / v\right)
$$

where $a_{n}$ is the amplitude of the wall reflection arriving at the microphone, $d_{n}$ is the corresponding propagation distance and $v=v(T)$ is the speed of sound in air which is a function of temperature $T$. Taking the Fourier transform of $c(t)$ leads to the room transfer function (RTF) given by

$$
C(f)=\sum_{n=0}^{N_{\mathrm{x}}} a_{n} e^{j 2 \pi f d_{n} / v}
$$

for frequency $f$. When the speed of sound changes, the time $\tau_{n}=d_{n} / v$ taken to travel distance $d_{n}$ changes. Let the original and perturbed speeds of sound be $v_{0}$ and $v$ corresponding to temperatures $T_{0}$ and $T$, respectively. The change in propagation time is given by

$$
\Delta \tau_{n}=d_{n}\left(\frac{1}{v}-\frac{1}{v_{0}}\right)
$$




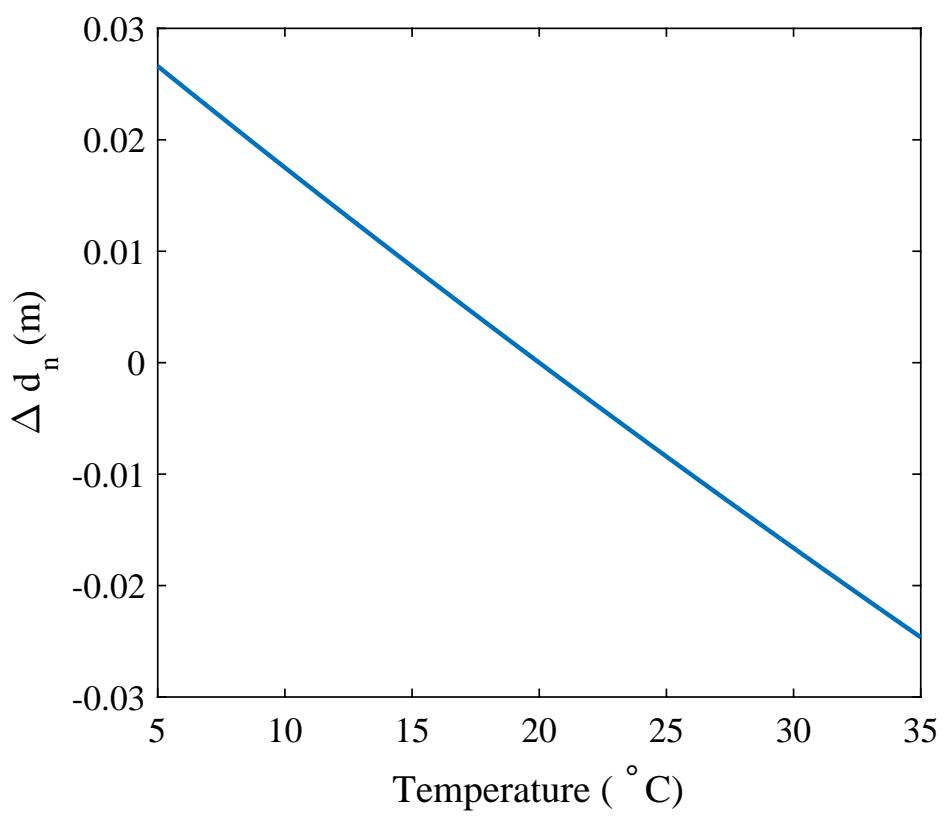

Figure 8.1: Variation in path length $\Delta d_{n}$ for a path length $d_{n}=1 \mathrm{~m}$ with temperature changes.

For a temperature increase (decrease), the propagation time is thus shortened (lengthened) and $\tau_{n}$ is negative (positive). Similarly, the change in path length corresponding to the change in temperature is given by

$$
\Delta d_{n}=c \Delta \tau_{n}=c d_{n}\left(\frac{1}{v}-\frac{1}{v_{0}}\right)
$$

and in terms of the number of wavelengths by

$$
\frac{\Delta d_{n}}{\lambda}=f d_{n}\left(\frac{1}{v}-\frac{1}{v_{0}}\right)
$$

i.e., the change in the path length of each propagation path, in fraction of wavelengths is hence proportional to both frequency $f$ and the original path length $d_{n}$. The variation in $\Delta d_{n}$ with temperature is shown in Figure 8.1. It can be seen that $\Delta d_{n}$ is inversely proportional to change in temperature.

When equalizing a room impulse response, performance can be degraded significantly if the path length changes by only a tenth of a wavelength [38], which corresponds to a $36^{\circ}$ change in phase. Thus, even a small change in path length 
has significant effect on cancelation performance. One mechanism for cancelling a reverberant reflection in a room is destructive interference. Here, a reflection is canceled by anti-phasing the signal with a signal of equal amplitude generated by a loudspeaker located within the path of the propagation.

Consider canceling a single tone sin $\omega t$ of frequency $\omega=2 \pi f$ at a single point by producing an anti-phase signal $\sin (\omega t+\pi)$. For a temperature-induced change $\Delta t$ in a propagation delay of an original signal, the resultant is

$$
\sin (\omega t-\omega \Delta t)+\sin (\omega t+\pi)=-2 \sin \left(\frac{\omega \Delta t}{2}\right) \cos \left(\frac{\omega t-\omega \Delta t}{2}\right)
$$

which has amplitude $2 \sin (\omega \Delta t / 2) \approx \omega \Delta t$. For a phase shift in the original tone of $36^{\circ}$ (or $\lambda / 10$ ), the tone is only suppressed by $4.2 \mathrm{~dB}$. A $20 \mathrm{~dB}$ tone suppression requires the phase shift to be $6^{\circ}$ or less (i.e. $\lambda / 60$ ). Rearranging (8.5), for a $20 \mathrm{~dB}$ suppression of an acoustic path,

$$
f=\frac{1}{60 d_{n}\left|v^{-1}-v_{0}^{-1}\right|}
$$

For only a $1 \mathrm{~m} / \mathrm{s}$ change in the speed of sound from $v_{0}=342 \mathrm{~m} / \mathrm{s}$ to $v=343 \mathrm{~m} / \mathrm{s}$ and propagation distance of $d_{n}=10 \mathrm{~m}$, which in a typical living room includes all first and some second order wall reflections, the $20 \mathrm{~dB}$ suppression of such a wall reflection is only possible up to $195 \mathrm{~Hz}$. Thus, the change in path length due to a small change in the speed of sound is significant as far as the early reverberant reflections of an impulse response are concerned.

The speed of sound changes according to the temperature and humidity, and is this relevant for AC-SFR. An ideal gas law model of the speed of sound originating, for dry air is

$$
v=20.03 \sqrt{K+T}
$$

where $T$ is temperature in degrees Celsius and $K=273.15$ is the temperature in degrees Kelvin at 0 degrees Celsius [4]. Substituting (8.8) into (8.5) gives

$$
\frac{\Delta d_{n}}{\lambda}=\frac{f d_{n}}{20.03}\left(\frac{1}{\sqrt{K+T}}-\frac{1}{\sqrt{K+T_{0}}}\right)
$$

where $T=T_{0}+\Delta T$ and $T_{0}$ is the original temperature. Taking a first order Taylor approximation in $\Delta T$, the change in the path length is

$$
\frac{\Delta d_{n}}{\lambda} \approx \kappa f d_{n} \Delta T
$$


where $\kappa=(20.03)^{2} / 2 v_{0}^{3}$. Thus, temperature induced perturbations to the path length are more extreme at higher frequencies and for longer propagation distances. This poses a problem for room compensation algorithms and any algorithm seeking to equalize the sound over a sizeable spatial sound zone. In the particular application of AC-SFR with higher order loudspeakers, sound bounces off walls so as to achieve spatial sound reproduction. Here certain sound propagations must be canceled over the zone of reproduction. These propagations could either be a direct source propagation or a wall reflection. The cancelation of such a propagation is achieved by destructive interference, i.e., by linearly combining and anti-phasing one propagating sound with another one. In a room, it is the higher order reflections that are more distant from the listener. These reflections are more susceptible to phase changes induced by a change in temperature. When it comes to performing AC-SFR then, it is possible to use a weak higher order reflection to anti-phase a stronger lower order reflection. However, the above analysis would suggest this approach is highly susceptible to temperature. Since a higher order reflection is more heavily influenced by temperature, the robustness of the cancelation approach utilizing higher order reflections is significantly reduced compared to lower order reflections. Therefore, it is less advisable to use higher order reflection to perform AC-SFR.

This chapter presents a method of optimizing the designed filters to reduce the utilization of higher order reflections in performing AC-SFR. We propose controlling the reflections which are utilized by adding a penalty function with a weighting on the different parts of the designed filters. Greater penalty shall be applied to the earlier parts of the filters, which are more susceptible to temperature. This will be shown to reduce the levels of pre-reverberation and penalty for the latter parts of the filters shall be relaxed.

\subsection{Sound Field Reproduction}

The task of sound field reproduction is to create an intended sound field around the listener using a number of surrounding loudspeakers. A common type of sound field is that of a virtual source located near the listener. To compensate for room reverberation, the RIRs from each loudspeaker to each point in the sound reproduction region must be completely characterized. The RIRs are captured using a microphone array - a pair of concentric arrays of microphones suspended in free space could be used, but more commonly, a single array is used with microphones 


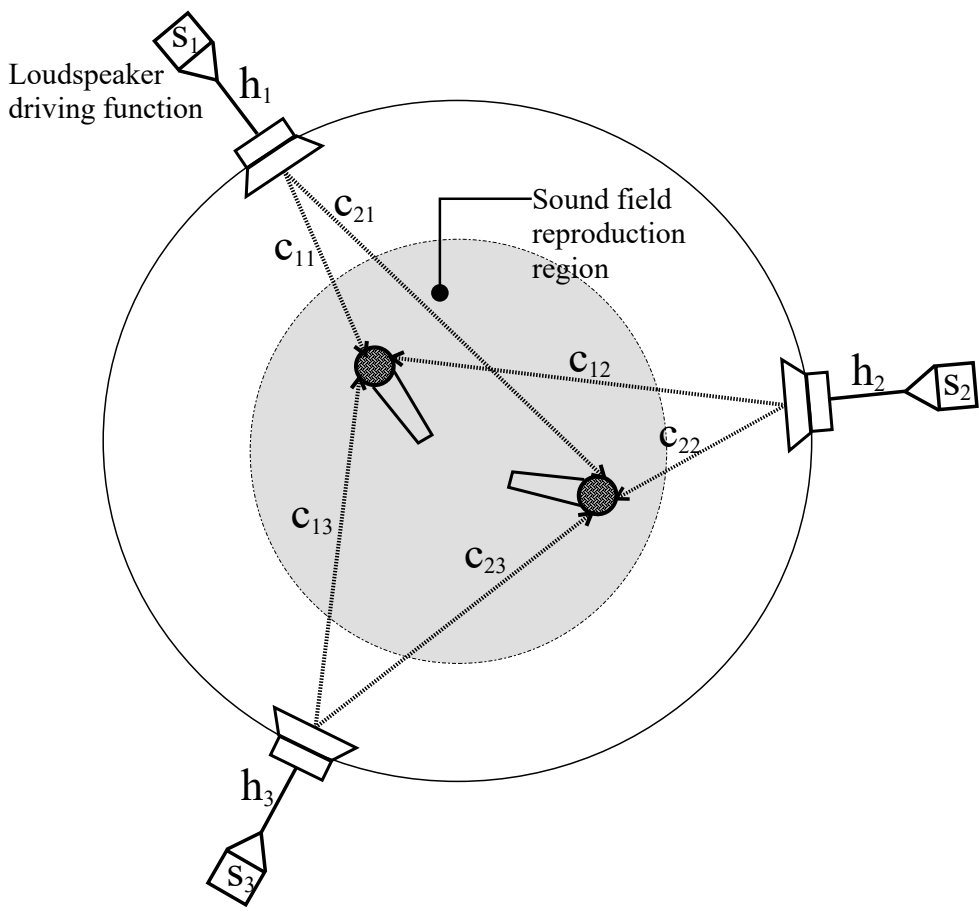

Figure 8.2: An example sound field reproduction setup showing the acoustic channels $c_{m l}$ and loudspeaker filters $h_{l}$.

mounted onto a solid baffle. In 2-D, it is sufficient to measure the RIRs to a circular array of microphones.

To reproduce a sound field over a cylindrical region of space, one can either match the sound pressure at a number of points, or match the spatial modes. The pressure matching formulation is discussed in this chapter. In pressure matching formulation, the sound pressure over a large set of points are set to be equal to a predefined sound pressure function. To understand the formulation, consider a simple setup with a microphone array of only $M=2$ sensors lying within a circular listening zone surrounded by $L=3$ loudspeakers as in Figure 8.2.

The matrix equation for this system can be written as

$$
\left[\begin{array}{l}
r_{1} \\
r_{2}
\end{array}\right]=\mathcal{C}\left[\begin{array}{l}
h_{1} \\
h_{2} \\
h_{3}
\end{array}\right]
$$


where $\mathcal{C}$ is the block Toeplitz matrix defined as

$$
\mathcal{C}=\left[\begin{array}{lll}
C_{11} & C_{12} & C_{13} \\
C_{21} & C_{22} & C_{23}
\end{array}\right]
$$

of dimension $N_{\mathrm{r}} M \times N_{\mathrm{h}} L$ where $N_{\mathrm{h}}$ is the length of each loudspeaker filter $h_{l}$ and $N_{\mathrm{r}}$ is the length of each desired response $r_{m}$ at microphone $m$. The Toeplitz convolution matrices $C_{m l}$ in $\mathcal{C}$ are each constructed from the channel impulse responses $c_{m l}$ from the $l$ th loudspeaker to $m$ th microphone. Typically, $N_{\mathrm{r}}$ and $N_{\mathrm{h}}$ are quite large.

In general, the pressure matching problem can be written in vector form in time domain as

$$
\mathcal{C} h=r
$$

where vector $h$ is a concatenated vector of loudspeaker filters $h_{l}$ and vector $r$ is the concatenated vector of desired impulse responses to be obtained at each of the microphones.

The desired sound pressure $r_{m}$ at each point of interest in the sound field is derived as follows. The sound pressure for an acoustic monopole at frequency $f$ is given by

$$
P_{d}\left(x_{m} ; f\right)=\frac{e^{-j 2 \pi f d_{m} / v}}{d_{m}}
$$

where $d_{m} \triangleq\left\|x_{m}-y_{v s}\right\|$, or in the time domain, we get

$$
p_{d}\left(t ; x_{m}\right)=\int_{-\infty}^{\infty} \frac{e^{j 2 \pi f\left(t-d_{m} / v\right)} d f}{d_{m}}=\frac{1}{d_{m}} \delta\left(t-d_{m} / v\right)
$$

When the signal for the desired sound field at each point is discretized or represented digitally using the inverse discrete Fourier transform,

$$
p_{d}\left(q ; x_{m}\right)=\frac{1}{2 \pi} \sum_{n=0}^{N-1} \frac{e^{j 2 \pi\left(q n / N-f_{n} d_{m} / v\right)}}{d_{m}}=\frac{1}{d_{m}} v \operatorname{csinc}_{N}\left(n \tau_{m}\right)
$$

where csinc is a circular sinc function [146], $\tau_{m}=F_{\mathrm{s}} d_{m} / v$ is the propagation delay of the virtual source to microphone $m$ in number of samples and $f_{n}=n F_{\mathrm{s}}=N_{\mathrm{r}}$ is the analog frequency. (Here, $N_{\mathrm{r}}$ is the length of GIR same as in Section 4.1.) Hence the desired response vector is

$$
r_{m}=\left[p_{d}\left(0 ; x_{m}\right), \cdots, p_{d}\left(N_{\mathrm{r}}-1 ; x_{m}\right)\right]^{\mathrm{T}}
$$




\subsubsection{Inverse filter solution}

The basic solution to the problem in (8.11) is the inverse filter solution, which finds the filter that best cancels the effect of $\mathcal{C}$ at the desired points. However, the designed filter coefficients may be large, making it difficult to implement the filters practically. This can be avoided by formulating the problem as a Tikhonovregularized optimization that additionally constrains the energy content in the filter coefficients $[147,148]$. The regularizer is added as a penalty function $\left(w_{\mathrm{tikh}}^{\mathrm{T}} h\right)^{2}$ with penalty weighting vector $w_{\text {tikh }}$. The penalty weighting decides the amount by which the filter coefficients are to be reduced. The typical inverse problem, thus, aims to minimize the objective function given by

$$
\jmath(h)=\frac{1}{2}\|\mathcal{C} h-r\|_{2}^{2}+\frac{1}{2}\left\|W_{\mathrm{tikh}} h\right\|_{2}^{2}
$$

where $W_{\text {tikh }}=\operatorname{diag}\left(w_{\text {tikh }}\right)$. This time domain formulation makes the matrix $\mathcal{C}$ quite large. Typically, one chooses the inverse filter in proportion to the length of the acoustic channels $N_{\mathrm{c}} \propto N_{\mathrm{h}}$. Matrix $\mathcal{C}$ then has $L N_{\mathrm{h}}$ columns and $M\left(N_{\mathrm{c}}+N_{\mathrm{h}}-1\right)=$ $M\left[(\alpha+1) N_{\mathrm{c}}-1\right] \approx(\alpha+1) M N_{\mathrm{c}}$ rows so that the number of elements in $\mathcal{C}$ is of order $M L N_{\mathrm{c}}^{2}$. However, for inverse filter design, the DFT (or equivalently, a frequency domain approach) is used to diagonalize the problem. For pressure matching over a large number of points, the matrix $\mathcal{C}$ is not stored in memory for channels of practical length; instead the DFT of each vector $c_{m l}$ is stored.

\subsubsection{Impulse response shaping solution}

As seen in previous chapters, impulse response shaping (IRS) is a more versatile approach than inverse filtering, allowing explicit control of pre-echo, early reflection and late reverberation. The approach designs loudspeaker filters by minimizing a weighted error function. This imposes less stringent constraints than inversion and hence results in an inherent robustness. The objective function for the IRS solution to the pressure matching problem of sound reproduction is given by

$$
\jmath(h)=\frac{1}{2}\left\|W_{1}(C h-r)\right\|_{2}^{2}+\frac{1}{2}\left\|W_{2} h\right\|_{2}^{2}
$$

where $W_{1}=\operatorname{diag}\left(w_{\mathrm{DR}}\right)$ is a diagonal weighting matrix on the desired microphone responses and $W_{2}=\operatorname{diag}\left(w_{\mathrm{tikh}}\right)$ is the diagonal weighting matrix on the loudspeaker filters. The weights $w_{\mathrm{DR}}$ were defined in equation 4.6 of Chapter 4 in accordance with the acoustic masking limit $[3,77,78] . w_{\mathrm{DR}}$ is defined based on a 
pre-echo time $N_{1}$ taps, a direct part and early reflections of $N_{2}$ taps followed by the late reverberation $N_{3}=N_{\mathrm{r}}-N_{1}-N_{2}$ taps as described in Chapter 4. However from (8.14), the propagation time from virtual source to each microphone location $x_{m}$ is now a function of the location. The position of the direct part, and hence the pre-echo time $N_{1}$ is now a function of the the microphone location for an acoustic monopole desired field in the pressure matching problem. A distinctive feature of the pressure matching problem is that these parameters are now a function of the pressure matching location.

The filter weight vector $w_{\text {tikh }}$ enables shaping of the loudspeaker filters to avoid placing of high levels of energy in the early parts of the filters which generates the pre-echo.

$$
w_{\mathrm{tikh}}=\left(1+\alpha \lambda_{\text {pre }}\right) \lambda_{\text {tikh }}
$$

where $\alpha$ is the pre-reverberation penalty factor, $\lambda_{\text {pre }}$ is a pre-reverberation penalty weighting vector and $\lambda_{\text {tikh }}$ is the Tikhonov regularization factor. $\lambda_{\text {pre }}$ is defined as an exponentially decaying function that strongly penalises the early part of the loudspeaker filters but applies no weight past the time index $n_{0}$ :

$$
\lambda_{\text {pre }}(n)=\left\{\begin{array}{cc}
\frac{e^{-\left(n-n_{0}\right) / \tau}-1}{e^{n_{0} / \tau}-1} & n \leq n_{0} \\
0 & n>n_{0}
\end{array}\right.
$$

The choice of $n_{0}$ and $\tau$ are based on the following considerations on the pre-echo:

- Assuming that the loudspeakers are approximately equidistant from the sound reproduction region, let $N_{\text {mdl }}(x)$ be the minimum propagation time from a loudspeaker to a point of interest $x$ in the sound reproduction region. The components of the loudspeaker filters contributing to pre-reverberation occur till $N_{1}+1$ taps minus the propagation time of $N_{\text {mdl }}$ taps. The loudspeaker penalty weight is thus applied on this region. The time of $n_{0}$ taps indicates the duration of time used to exploit room reflections. For example, an $n_{0}$ of $30 \mathrm{~ms}$ implies a propagation distance of $10.2 \mathrm{~m}$ which typically includes first order and some second order reflections in small rooms.

- Decay rate parameter $\tau$ determines how aggressively the early energy in loudspeaker filters is penalised in relation to late energy. To penalise all of these components equally, one would set $\tau$ large.

The loudspeaker weighting function is used in conjunction with Tikhonov regularization to ensure that the designed filters maintain causality. 


\subsection{Relevant Solutions}

The impulse response shaping formulation is solved using three different algorithms specifically, steepest descent method, conjugate gradient descent method (CG) and a variant of the Fast DALM algorithm D-2-2 derived in Chapter 5. The performance of the algorithms in the pressure matching problem are compared in Section 8.6.

\subsubsection{Steepest Descent Method}

The steepest descent method [85] solves the problem iteratively using the update step

$$
h_{i+1}=h_{i}-\mu \frac{\partial \jmath}{\partial h_{i}}
$$

where the gradient vector of the objective function $\partial \jmath / \partial h_{i}$ is given by differentiating (8.19):

$$
\frac{\partial \jmath}{\partial h}=\left(\mathcal{C}^{\mathrm{T}} W_{1}^{\mathrm{T}} W_{1} \mathcal{C}+W_{2}^{\mathrm{T}} W_{2}\right) h-\left(\mathcal{C}^{\mathrm{T}} W_{1}^{\mathrm{T}} W_{1} r\right)=\mathcal{C}^{\mathrm{T}} W_{1}^{2}(\mathcal{C} h-r)+W_{2}^{2} h
$$

The convergence of this algorithm depends on the proper choice of the step size parameter [88]. The convergence rate of steepest descent method is generally linear. In this work, the steepest descent is implemented using conjugate gradients and approximate line searches based on polynomial interpolation with Wolfe-Powel conditions [149].

\subsubsection{Conjugate Gradient Descent Algorithm}

The conjugate gradient (CG) method [93] is also applied to solve the pressure matching problem. The advantage of $\mathrm{CG}$ method over steepest descent is that it converges quickly since the step direction is made to be orthogonal to the previous step directions. The objective function in (8.19) can be rewritten as

$$
\jmath(h)=\frac{1}{2}\left\|\left[\begin{array}{c}
W_{1} \mathcal{C} \\
w_{\text {tikh }}^{\mathrm{T}}
\end{array}\right] h-\left[\begin{array}{c}
W_{1} r \\
0
\end{array}\right]\right\|_{2}^{2}
$$

It can be seen that the new matrix $A \triangleq\left[\mathcal{C}^{\mathrm{T}} W_{1}^{\mathrm{T}} \quad w_{\text {tikh }}\right]^{\mathrm{T}}$ is rectangular. The problem can be formulated as $\min _{h}\left\|A^{\mathrm{T}} A x-A^{\mathrm{T}} b\right\|_{2}^{2} / 2$ where $x=h$ and $b=$ 
$\left[\begin{array}{ll}r^{\mathrm{T}} W_{1}^{\mathrm{T}} & 0\end{array}\right]^{\mathrm{T}}$. Now, the CG method can be easily applied to minimize the error $A^{\mathrm{T}} A x-A^{\mathrm{T}} b$. The algorithm can be summarized as follows:

Step 1: Initialize $x_{0}$

$$
\begin{aligned}
\text { Step 2: } & r_{0}=A^{\mathrm{T}} b-A^{\mathrm{T}} A x_{0} \\
& =\mathcal{C}^{\mathrm{T}} W_{1}^{2} r-\left(\mathcal{C}^{\mathrm{T}} W_{1}^{2} \mathcal{C} h_{0}+w_{\text {tikh }} w_{\text {tikh }}^{\mathrm{T}} h_{0}\right) \\
& p_{0}=r_{0} \\
k & =0
\end{aligned}
$$

Step 3: Repeat

$$
\begin{aligned}
& \alpha_{k}=\frac{r_{k}^{\mathrm{T}} r_{k}}{p_{k}^{\mathrm{T}} A^{\mathrm{T}} A p_{k}}=\frac{r_{k}^{\mathrm{T}} r_{k}}{p_{k}^{\mathrm{T}}\left(\mathcal{C}^{\mathrm{T}} W_{1}^{2} \mathcal{C} p_{k}+w_{\mathrm{tikh}} w_{\mathrm{tikh}}^{\mathrm{T}} p_{k}\right)} \\
& x_{k+1}=x_{k}+\alpha_{k} p_{k} \\
& r_{k+1}=r_{k}-\alpha_{k} p_{k} \\
& \text { If } r_{k+1} \text { is small, exit loop } \\
& \beta_{k}=\frac{r_{k+1}^{\mathrm{T}} r_{k+1}}{r_{k}^{\mathrm{T}} r_{k}} \\
& p_{k+1}=r_{k+1}+\beta_{k} p_{k} \\
& k=k+1 \\
& \text { End repeat }
\end{aligned}
$$

The algorithm generally converges in $n$ steps, where $n$ is the number of distinct eigenvalues of the matrix $C$.

\subsubsection{Variant of Fast D-2-2 algorithm}

The pressure matching problem requires the DALM algorithm to solve an $\ell_{2}$ regularized $\ell_{2}$ minimization problem of the form of (8.19). The derivation is similar to the approaches presented in Chapter 5 and Appendix A, by finding the Fenchel conjugates and using the Fenchel duality theorem. The dual augmented Lagrangian corresponding to the primal problem (8.19) is

$$
\begin{aligned}
& \min _{y, z} \frac{1}{2} y^{\mathrm{T}} y-W r^{\mathrm{T}} y+\frac{1}{2} z^{\mathrm{T}} W_{3} z \\
& -h^{\mathrm{T}}\left(z-\mathcal{C}^{\mathrm{T}} W_{1}^{\mathrm{T}} y\right)+\frac{\beta}{2}\left\|z-\mathcal{C}^{\mathrm{T}} W_{1}^{\mathrm{T}} y\right\|_{2}^{2}
\end{aligned}
$$

where $y, z$ are the dual variables and $0<\beta \leq 1$ decides the amount of augmentation applied. $W_{3}$ is a diagonal matrix with elements $1 / w_{\text {tikh }}^{2}$. The update equations for $h, y$ and $z$ are found from the derivative of the dual Lagrangian with respect 
to each of the corresponding variables. As seen in previous chapters, Fast DALM [34] for this problem is found by replacing the $y$-update by one step of the CG algorithm and can be summarised as

$$
\begin{array}{ll}
\text { Step 1: } & z_{k+1}=\left(W_{3}+\beta I\right)^{-1}\left(\beta \mathcal{C}^{\mathrm{T}} W_{1}^{\mathrm{T}} y+h\right) \\
\text { Step 2: } & g_{k}=y_{k}-W r+\beta W_{1} \mathcal{C}\left(W_{1} \mathcal{C}\right)^{\mathrm{T}} y_{k}-\beta W_{1} \mathcal{C} z_{k+1}+W_{1} \mathcal{C} h_{k} \\
& \alpha_{k}=g_{k}^{\mathrm{T}} g_{k} /\left(\lambda g_{k}^{\mathrm{T}} g_{k}+\beta g_{k}^{\mathrm{T}}\left(W_{1} \mathcal{C}\right)\left(W_{1} \mathcal{C}\right)^{\mathrm{T}} g_{k}\right) \\
& y_{k+1}=y_{k}-\alpha_{k} g_{k}
\end{array}
$$

Step 3: $\quad h_{k+1}=h_{k}-\beta\left(z_{k+1}-\left(W_{1} \mathcal{C}\right)^{\mathrm{T}} y_{k+1}\right)$

The convergence of DALM algorithm is generally super-linear; the Fast DALM algorithm further improves the convergence due to the CG step replacing the matrix inversion.

\subsection{Forward Adjoint Oracle Approach}

Forward Adjoint Oracle Approach (FAO) [108] implementations are similar to those presented in previous chapters using FFT operations. This is possible because the $W_{1} \mathcal{C}$ matrix is a Toeplitz matrix representing convolution.

\subsubsection{Implementation of $\mathcal{C} h$ using Fast Fourier Transforms}

The implementation of $\mathcal{C} h$ using FAO is same as the procedure mentioned in 5.5 in Chapter 5. It is implemented as

$$
r_{m}=\mathcal{F}^{-1}\left\{\sum_{l}\left(\mathcal{F}\left\{\check{c}_{m l}\right\} \odot \mathcal{F}\left\{\check{h}_{l}\right\}\right)\right\}
$$

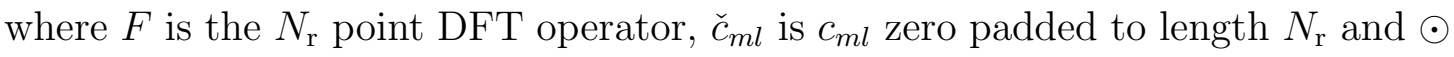
represents Hadamard product. This operation consists of only forward and inverse fast Fourier transform operations, vector multiplications and summation along $L$ loudspeakers. Thus, the complexity $\mathcal{O}\left(N_{\mathrm{r}}^{2}\right)$ of one matrix multiplication has been reduced to $\mathcal{O}\left(N_{\mathrm{r}} \log \left(N_{\mathrm{r}}\right)\right)$ using this implementation. 


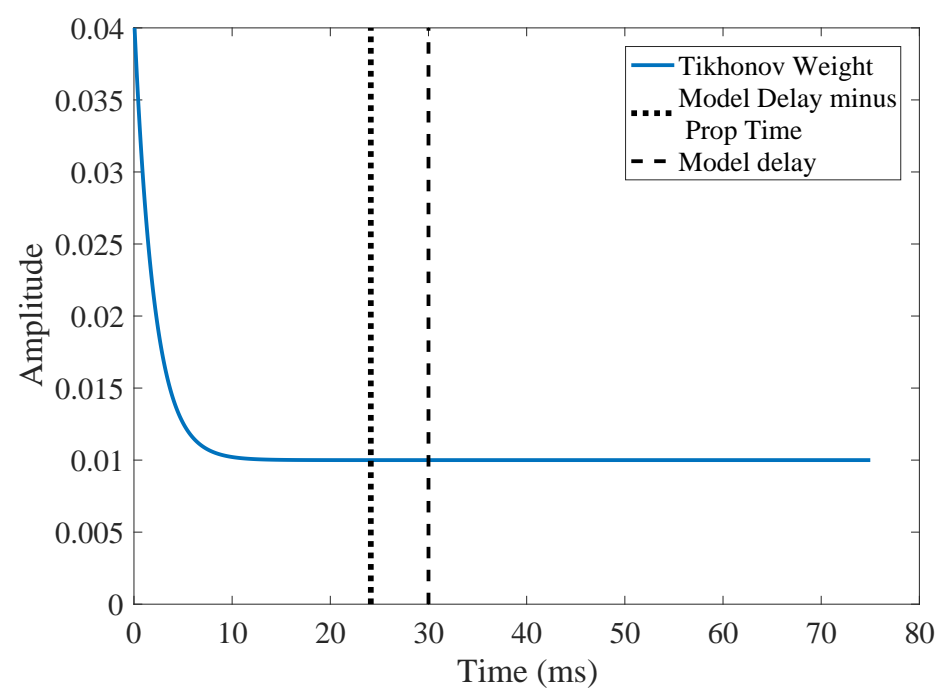

Figure 8.3: Loudspeaker filter weights for a $30 \mathrm{~ms}$ modeling delay and a $2 \mathrm{~m}$ loudspeaker-to-microphone distance. Here, $\lambda_{\text {tikh }}=0.01$ and $\alpha=4$.

\subsubsection{Implementation of $\mathcal{C}^{\mathrm{T}} y$ using Fast Fourier Transforms}

Using the same principle as the previous section, $\mathcal{C}_{m l}^{\mathrm{T}} y_{m}$ can be represented as $\mathcal{C}_{m l}^{\mathrm{T}} y_{m}=\left(F^{-1} D_{m l} F\right)^{\mathrm{T}} y_{m}=F^{\mathrm{T}} D_{m l}\left(F^{\mathrm{T}}\right)^{-1} y_{m}$ where $D_{m l}$ is a diagonal matrix of the FFT values of $C_{m l}$. This is equivalent to performing $\mathcal{F}\left\{\mathcal{F}\left\{\check{c}_{m l}\right\} \odot \mathcal{F}^{-1}\left\{y_{m}\right\}\right\}$. Therefore, $C^{\mathrm{T}} y$ can be written as

$$
\check{h}_{l}=\mathcal{F}\left\{\sum_{m}\left(\mathcal{F}\left\{\check{c}_{m l}\right\} \odot \mathcal{F}^{-1}\left\{y_{m}\right\}\right)\right\}
$$

which results in a vector $\check{h}_{l}$ of length $N_{\mathrm{r}}$. The desired result is obtained by truncating $\check{h}_{l}$ to length $N_{\mathrm{h}}$. This truncation does not cause any error in $\check{h}_{l}$ since $c_{m l}$ has been zero padded to length $N_{\mathrm{r}}$ and the terms removed are the results of terms of $y$ multiplied by the added zeroes. This is equivalent to deleting $N_{\mathrm{h}}$ rows from the end of matrix $F$. Here again, the complexity $\mathcal{O}\left(N_{\mathrm{r}}^{2}\right)$ has been reduced to $\mathcal{O}\left(N_{\mathrm{r}} \log \left(N_{\mathrm{r}}\right)\right)$ using this implementation.

\subsection{Simulation results}

Simulation studies were performed for a room of dimension $6.4 \times 5 \times 4 \mathrm{~m}$ with a wall reflection coefficient 0.7 and a sampling frequency of $16 \mathrm{kHz}$. The $T_{60}$ of 


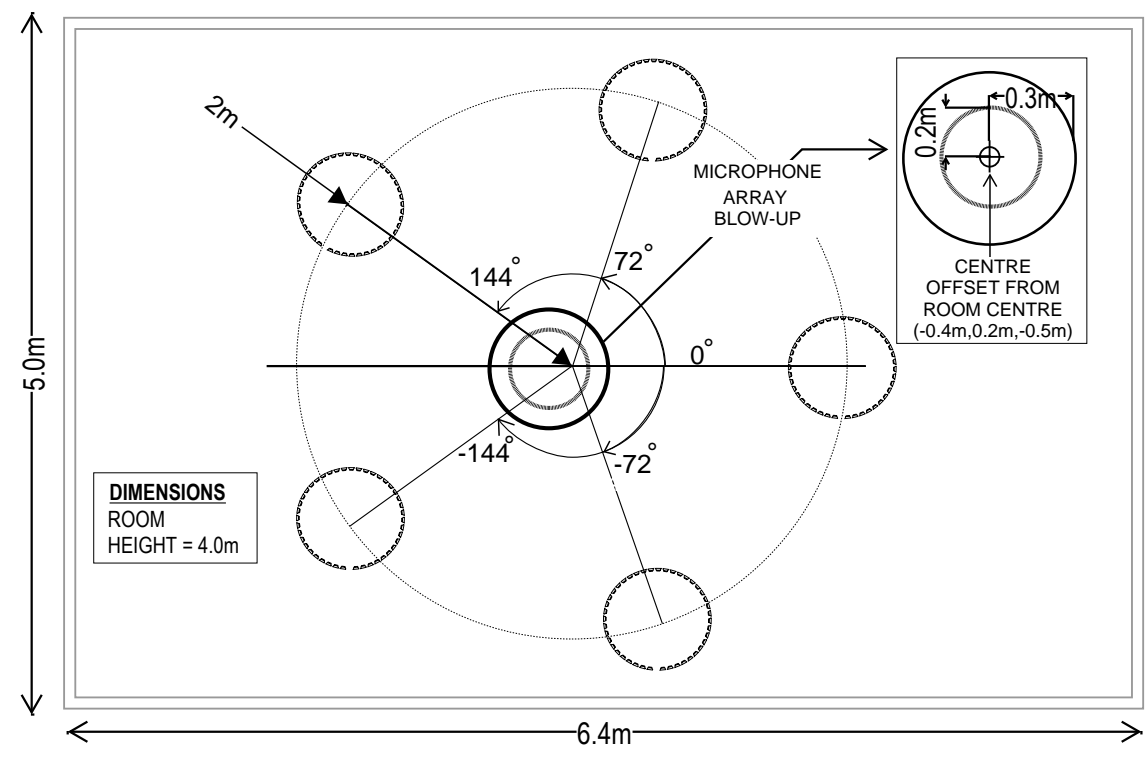

Figure 8.4: Layout of the pressure matching setup used in the simulations.

the room was $186 \mathrm{~ms}$. To compute the loudspeaker filters, the channels used were truncated to a length $N_{\mathrm{c}}=1200$ and shaping filters of length $N_{\mathrm{h}}=4800$ and a delay $N_{\mathrm{d}}=1102$. The loudspeakers were arranged as 5 circular arrays each with 8 loudspeaker drivers. The arrays were of radius $0.15 \mathrm{~m}$ each circular array being located $2 \mathrm{~m}$ from the centre of the listening region, at angles $0^{\circ}, \pm 72^{\circ}, \pm 144^{\circ}$. Such an arrangement was used to represent a monopole source. The microphones were arranged as 2 concentric spherical arrays with radii $0.2 \mathrm{~m}$ and $0.3 \mathrm{~m}$ respectively, each with 100 microphones arranged in a 100 point Fliege geometry. The spherical reproduction region has a centre located at $(-40,20,-50) \mathrm{cm}$ relative to the centre of the room. A layout of the setup used is shown in Figure 8.4. The modelling delay to direct path, 'don't care' regions and post-reverberation regions were set as $30 \mathrm{~ms}, 10 \mathrm{~ms}$ and $146 \mathrm{~ms}$ respectively. The weighting vector $w_{1}$ was set according to [39] to match the temporal masking limit. The weight vector $w_{2}$ was set according to (8.20) with $\alpha=4, \beta=12$ and $\lambda_{\text {tikh }}=0.01$. The filter weight vector $w_{2}$ applied to the loudspeaker filters in this experiment is shown in Figure. 8.3.

The performance metric used is the direct to reverberation energy ratio (DRR) which quantifies the reverberation reduction performance. The DRRs are computed separately for pre-reverberation $\left(\gamma_{\text {pre }}\right)$ and post-reverberation $\left(\gamma_{\text {post }}\right)$ using a 3 ms raised cosine window to clip the direct part, and windows with $1.5 \mathrm{~ms}$ co- 


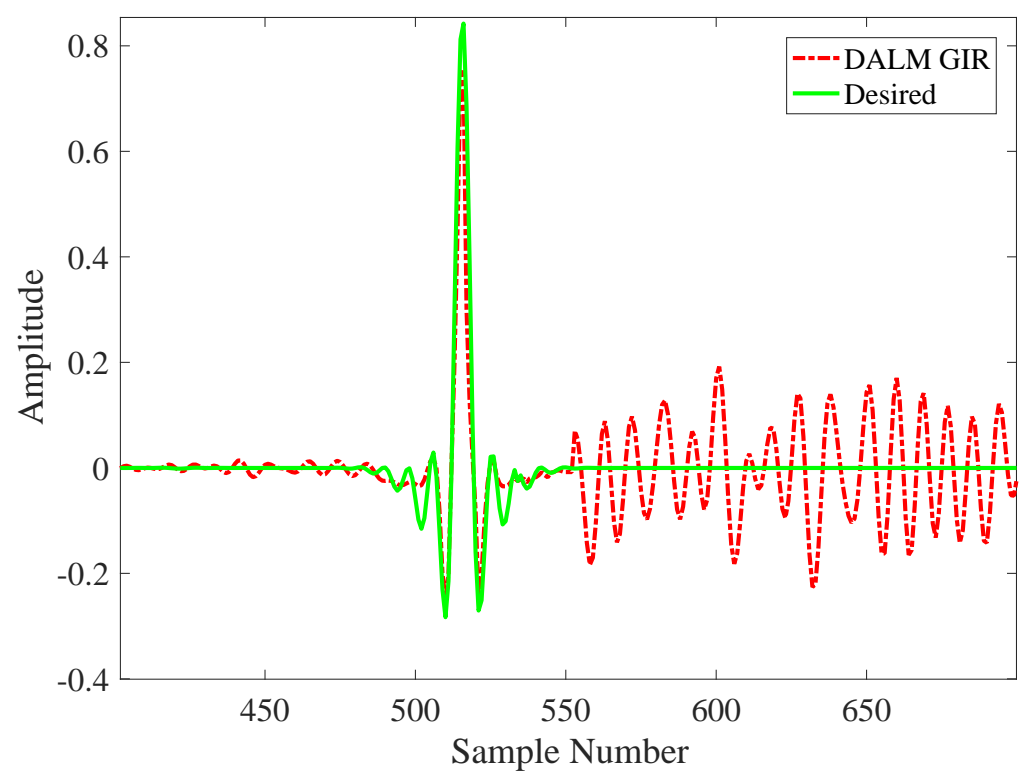

Figure 8.5: One of the Global Impulse Responses (GIR) obtained using the shaping algorithm based on DALM in a $6.4 \times 4 \times 3 \mathrm{~m}$ room with wall reflection coefficient of 0.7 at $20^{\circ} \mathrm{C}$.

sine roll off to clip the pre-reverberation and post-reverberation in the vicinity the direct component for all the three shaping algorithms and inversion to compare the performance.

All the three shaping algorithms namely steepest descent, CG and Fast DALM as well as the inversion method were tested for their performance in the pressure matching problem. A simulation comparing inversion and shaping algorithms was performed at two different temperatures. In order to quantify the temperature robustness, a design was performed at $20^{\circ} \mathrm{C}$ and tested at $24^{\circ} \mathrm{C}$. One of the Global Impulse Responses (GIR) obtained with the shaping algorithms at a designed temperature of $20^{\circ} \mathrm{C}$ and the same test temperature are shown in Figure. 8.5. At the designed temperature, it can be seen that the responses show low prereverberation and post-reverberation, with a peak at the direct path. The GIR obtained at the perturbed temperature of $24^{\circ} \mathrm{C}$ is shown in Figure. 8.6.

The response obtained with inverse filtering is shown in Figure. 8.7 and Figure. 8.8. It can be seen that there is significant pre- and post-reverberation. This 


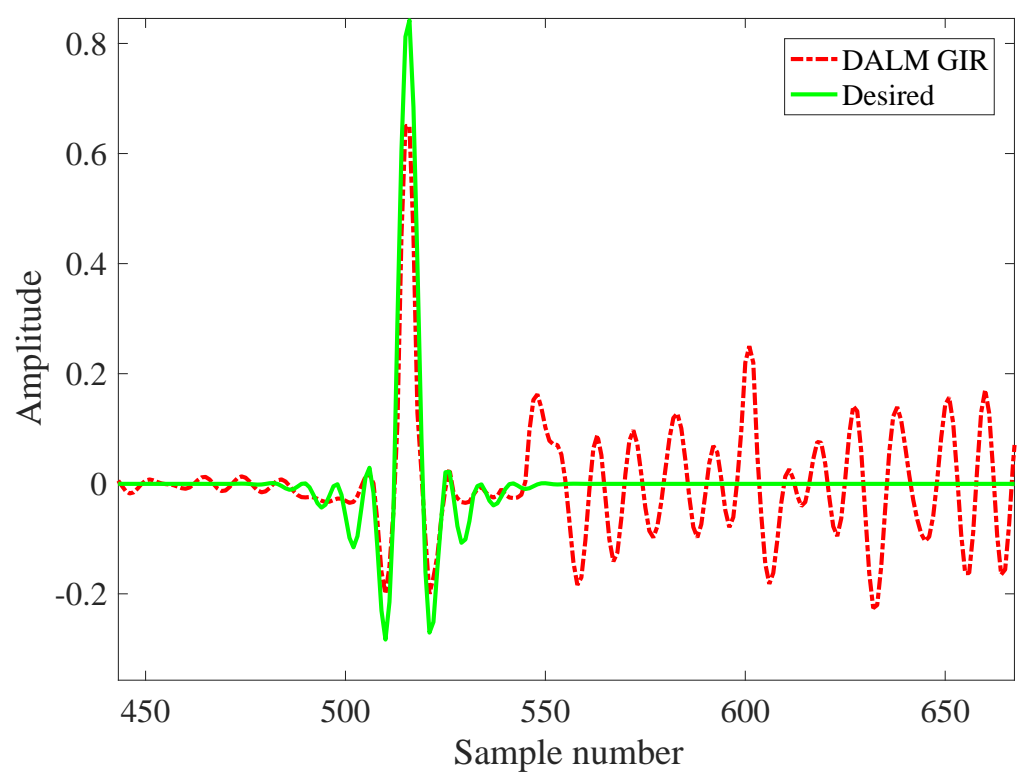

Figure 8.6: One of the Global Impulse Responses (GIR) obtained using the shaping algorithm based on DALM at $24^{\circ} \mathrm{C}$.

could be reduced using a longer inverse filter and smaller regularization parameter (currently $10^{-2}$ ). At the test temperature, the performance is poor with significant pre and post reverberations as in Figure. 8.8. Thus, inversion performs better than shaping when the designed and test conditions are the same.

The frequency responses of the shaping approaches and inverse filter are shown in Figure. 8.9 and Figure. 8.10. By comparing these figures, it can be seen that the frequency response of the shaping approaches do not vary much with temperature change, but the frequency response with inversion becomes increasingly noisy at higher frequencies for a temperature change. In addition, among shaping algorithms, the responses with CG and DALM are similar.

The performance of the shaping algorithms in this problem is tabulated in Table 8.1. It can be seen from Table 8.1 that CG converges to a lower objective value; but at the cost of higher computation time. Both SD and DALM algorithms were run with a fixed number of iterations for this problem (240 for SD, 2000 for DALM). It was observed that DALM iterations produce a solution close to CG in less computation time. Since the problem is convex, all the algorithms should 


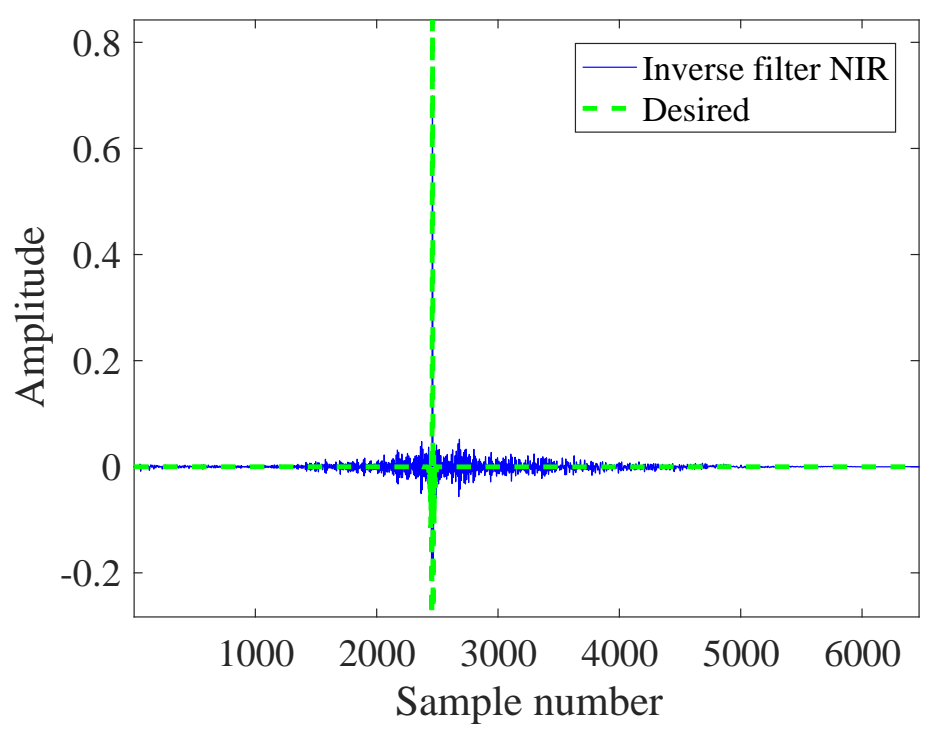

Figure 8.7: One of the Nett Impulse Responses (NIR) for loudspeaker driver 1 to microphone 1 obtained with inversion at $20^{\circ} \mathrm{C}$.

Table 8.1: Performance of the shaping algorithms at the designed temperature. Performance metrics CPU time and $\gamma$ are defined as in Section 4.4. Objective function value is the final value of the objective in (8.19) obtained using the different algorithms.

\begin{tabular}{cccccc}
$\begin{array}{c}\text { Temperature } \\
\circ\end{array}$ & Algorithm & $\begin{array}{c}\text { CPU Time } \\
(\mathrm{s})\end{array}$ & $\begin{array}{c}\text { Objective } \\
\text { value }\end{array}$ & $\begin{array}{c}\gamma_{\text {pre }} \\
(\mathrm{dB})\end{array}$ & $\begin{array}{c}\gamma_{\text {post }} \\
(\mathrm{dB})\end{array}$ \\
\hline 20 & $\mathrm{SD}$ & 3205 & 45.23 & 14.3 & -2.1 \\
\hline 20 & $\mathrm{CG}$ & 7778 & 0.0546 & 18.4 & -8.7 \\
\hline 20 & $\mathrm{DALM}$ & 652 & 0.0755 & 15.6 & -6.2 \\
\hline 24 & $\mathrm{SD}$ & 3207 & 44.16 & 14.4 & -2.1 \\
\hline 24 & $\mathrm{CG}$ & 7780 & 0.052 & 18.2 & -8.6 \\
\hline 24 & $\mathrm{DALM}$ & 652 & 0.0641 & 15.6 & -6.2
\end{tabular}

converge to the same solution; but SD may take a long time to achieve this. Allowing DALM iterations to run until convergence may take slightly more time than $652 \mathrm{~s}$, close to $900 \mathrm{~s}$. The iterations were stopped at this stage because a solution 


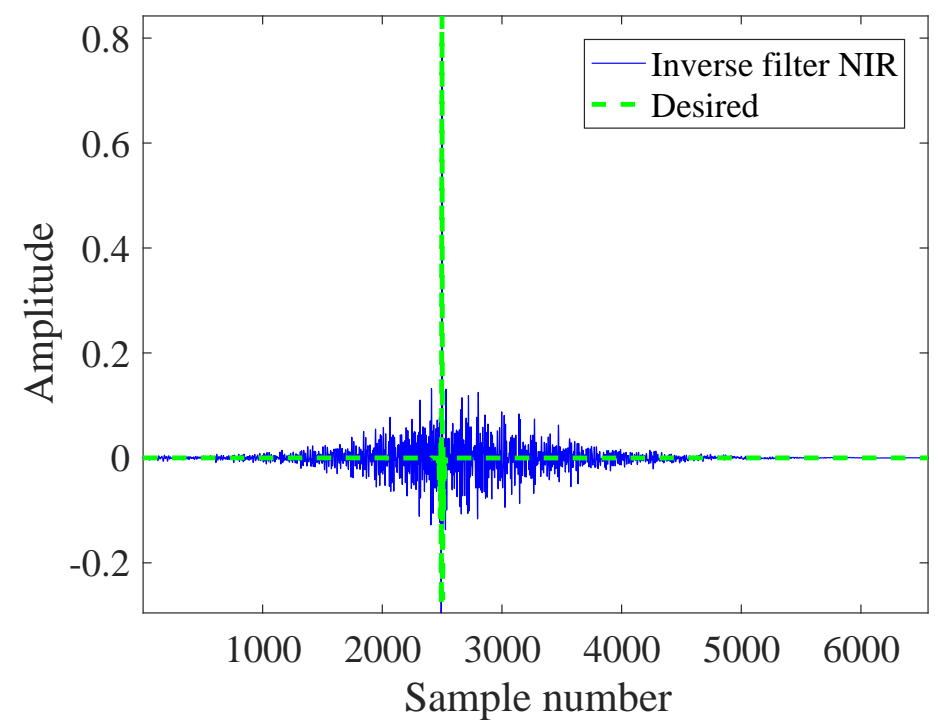

Figure 8.8: One of the Nett Impulse Responses (NIR) for loudspeaker driver 1 to microphone 1 obtained with inversion at $24^{\circ} \mathrm{C}$.

close to the minimum was obtained with a good DRR performance. Evaluating the optimization performance using a combination of objective and computation time shows that DALM performs the best for this problem, then CG and finally SD.

Another interesting observation from the results in Table 8.1 is that the DRR values $\gamma_{\text {pre }}$ and $\gamma_{\text {post }}$ for the IRS methods do not vary much with change in temperature. Among these, the values are highest for CG method, indicating its better optimization performance. But it can be seen that the computation time taken is higher to converge to an objective close to the solution provided by DALM. DALM gives a slightly lower DRR but in a computationally much faster manner. The DRR values obtained for inversion was found to be $\gamma_{\text {pre }}=16.4 \mathrm{~dB}$ and $\gamma_{\text {post }}=12.6 \mathrm{~dB}$ at $20^{\circ} \mathrm{C}$. Thus, the DRR values are higher for inversion than DALM and SD solutions at the designed temperature. This is as expected, since inversion gives best performance at the designed conditions.

In order to check the temperature robustness, the designed filters were tested at a different test temperature. The DRR values for this test are tabulated in Table 8.2 .

Results in Table 8.2 show that the DRR values drop drastically for the inverse 


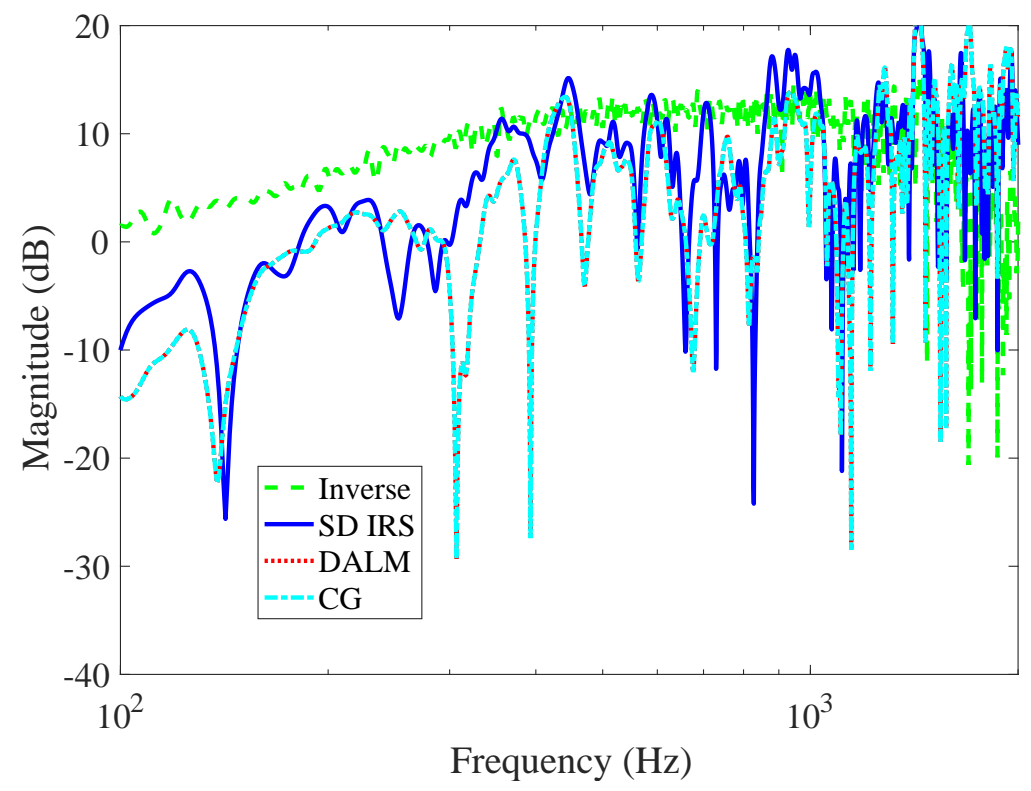

Figure 8.9: Frequency responses of the GIRs obtained with shaping methods and inversion at $20^{\circ} \mathrm{C}$.

Table 8.2: DRR performance when the test temperature is varied. Design temperature is $20^{\circ} \mathrm{C}$.

\begin{tabular}{ccccccc} 
Algorithm & $\begin{array}{c}\gamma_{\text {pre }}(d B) \\
\text { at } 18^{\circ} \mathrm{C}\end{array}$ & $\begin{array}{c}\gamma_{\text {post }}(\mathrm{dB}) \\
\text { at } 18^{\circ} \mathrm{C}\end{array}$ & $\begin{array}{c}\gamma_{\text {pre }}(d B) \\
\text { at } 22^{\circ} \mathrm{C}\end{array}$ & $\begin{array}{c}\gamma_{\text {post }}(\mathrm{dB}) \\
\text { at } 22^{\circ} \mathrm{C}\end{array}$ & $\begin{array}{c}\gamma_{\text {pre }}(d B) \\
\text { at } 24^{\circ} \mathrm{C}\end{array}$ & $\begin{array}{c}\gamma_{\text {post }}(\mathrm{dB}) \\
\text { at } 24^{\circ} \mathrm{C}\end{array}$ \\
\hline $\mathrm{SD}$ & 14.1 & -2.5 & 14.3 & -2.3 & 14.4 & -2.4 \\
\hline $\mathrm{CG}$ & 18.3 & -9.3 & 18.4 & -8.75 & 18.4 & -8.9 \\
\hline DALM & 15.3 & -6.7 & 15.8 & -6.25 & 15.8 & -6.4 \\
\hline Inversion & 7.8 & 4.2 & 8.1 & 3.4 & 5.9 & 0.4 \\
\hline
\end{tabular}

filtering solution when tested at a different temperature. This matches with the theoretical observation that inverse filter solution finds an exact inverse but is not robust to changes in channel. Coming to the shaping solutions, the DRR values vary only marginally for all the three algorithms, highlighting their temperature robustness. The post-reverberation levels of these algorithms are low, because the GIR weight function chosen concentrates on the cancellation of pre-reverberation. 


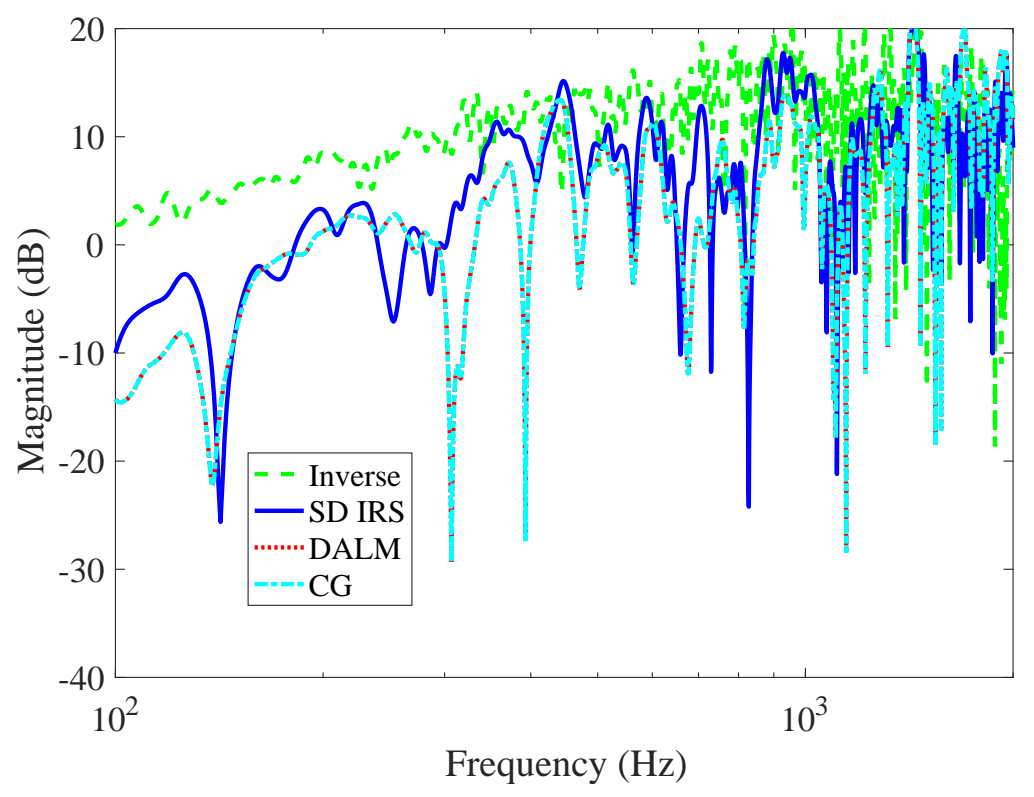

Figure 8.10: Frequency responses of the GIRs obtained with shaping methods and inversion at $24^{\circ} \mathrm{C}$.

From the analysis of the results, it can be seen that IRS method presented provides a better temperature robustness than inversion. Among the IRS solutions, the DALM solution provides the best performance with good reverberation cancelation in a low computation time.

\subsection{Contributions from this chapter}

To conclude, this chapter presented an application of the impulse response shaping formulation developed in this thesis. The shaping algorithm was applied to pressure matching of an active compensated sound field reproduction system to achieve an inherent robustness to change in temperature. The shaping solution was compared to the standard Tikhonov regularized inverse filtering scheme to analyse the robustness performance. The shaping algorithm was solved using three solution algorithms viz., steepest descent, conjugate gradient descent and fast DALM algorithm. The fast DALM algorithm developed in previous chapters was slightly modified to solve the pressure matching formulation. It has been observed from the simulation results that the fast DALM algorithm is computationally more efficient 
than CG and SD, providing similar results in low computation time. The computation time presented in Section 8.6 is the time taken to execute the shaping algorithm with no prior knowledge of the solution. In the case of a temperature change, the optimum solution will vary by only a small amount. Since the optimization problem is convex (i.e., there are no local minima in which the solution could be trapped), re-running the algorithm with the previous solution as the initialization should converge rapidly to this revised minimum. In this way, an AC-SFR implementation that can adapt to temperature changes in real time should be feasible. Validation of this idea is an obvious direction for future research. Thus the major contribution of this chapter is the application of impulse response shaping to AC-SFR problem and the development of the fast DALM algorithm to obtain a computationally fast solution to the problem. 


\section{Chapter 9}

\section{Conclusion}

The thesis presents a new approach for Acoustic Impulse Response Shaping suitable for joint shaping and cross-talk cancelation in multi-channel systems. The shaping approach utilizes a set of pre-filters, which are positioned before the loudspeakers (in a typical signal path in a room from source to loudspeakers, and then to the listeners) to regulate the reverberations at the listening positions. Addressing the fact that the room impulse responses (which form the basis for designing the pre-filters) are typically long, resulting in a large amount of data, the thesis has specifically concentrated on the development, realization and demonstration of computationally fast and memory efficient algorithms to design optimal filters. This improvement has been achieved using a regularized optimization formulation, which was solved using an algorithm known as Dual Augmented Lagrangian Method (DALM), known in the sparse reconstruction literature for its super-linear convergence. A computationally faster version of DALM, called Fast DALM was utilized in the thesis for computational efficiency. Memory efficiency was achieved using implementations based on a Forward Adjoint Oracle (FAO).

Though impulse response shaping is performed in time domain, additional frequency response control is required in practice to avoid large frequency response fluctuations. The thesis presents an approach to achieve joint frequency control by implementing this as a projection step which can be easily incorporated into the fast time shaping algorithm developed. Computationally fast versions of the projection algorithm were developed to reduce the impact of this additional step on the computational speed of the original algorithm. Thus, a fast approach for joint time and frequency shaping as well as cross-talk cancelation has been developed in this thesis. 
As discussed earlier, the shaping approach depends on the room response measurements which can vary due to measurement errors, atmospheric changes and changes in position inside the room. Therefore, robustness to channel variations is essential for a practical implementation of s shaping system. This thesis presents two approaches for achieving spatial robustness, one being an empirical design approach in which shaping filters robust to a number of perturbed positions around the original position are designed. This approach is based on a method available in literature, but we have improved the computational efficiency by making use of convex formulations and the DALM algorithm. Another spatially robust approach developed in this thesis provides statistical robustness, by probabilistically constraining large deviations from desired responses. This approach was seen to provide better robustness than the empirical approach, but this algorithm could not be developed into a practically realizable shaping system due to the difficulty in finding suitable computationally fast solution algorithms. The statistically robust problem turned out to be a second order cone program, and the difficulty in finding a fast and memory efficient algorithm for solving prevented us from developing it further as a part of this thesis.

A final contribution from this work is the application of shaping algorithms to an active compensated sound field reproduction problem. The shaping approach was specifically applied to achieve temperature robustness which is an issue with an AC-SFR. The approach was found to work well, providing better temperature robustness compared to a traditional inverse filtering scheme. This application highlights the versatility of the algorithm developed as a part of this thesis and its applicability to a wide range of problems.

A summary of specific contributions from each chapter are listed in the following section.

\subsection{Original contributions}

CHAPTER 5: The major contributions from this chapter were the development of fast algorithms for impulse response shaping. The algorithms developed were variants of the Dual Augmented Lagrangian Method, known in sparse reconstruction. The fast DALM algorithm (a computationally fast version of DALM) for solving an $\ell_{1}$ regularized $\ell_{2}$ norm minimization was applied to the shaping problem and good 
performance results were obtained in a computationally fast manner. Therefore, DALM algorithms to solve some other regularized formulations that can be useful for the problem specifically, $\ell_{2}$ regularized $\ell_{2}$ norm minimization, $\ell_{1}$ regularized $\ell_{\infty}$ norm minimization and $\ell_{2}$ regularized $\ell_{\infty}$ norm minimization were developed. One of the key contributions was the development of a fast DALM algorithm for solving the $\ell_{1}$ regularized $\ell_{\infty}$ norm minimization problems, which is most advantageous for the development of a shaping implementation in a real scenario. The efficacy of the fast DALM algorithms in shaping was verified using simulation and real time experimental studies. It was found from the experimental studies that each DALM iteration took only a few milliseconds and most of the computation time was spent in calculating the FFT of the vectors. This posed a limitation on improving the speed of the algorithms further, since there was really no way to improve the speed of FFT in Matlab. However, a possible improvement would have been to make the filter design adaptive.

Adaptive design can be achieved by performing the DALM iterations from the initial point only for the first design in a room and continuing the iterations from the previous optimum in the iteration for the subsequent channel perturbations. This could be a good solution because we assume that the minimum point only shifts to a nearby location due to perturbations and the iterations can still converge to the new minimum even if we start from an intermediate iteration of the first design. Such an improvement is possible primarily due to the convexity of our formulation, which avoids any chance of the solution getting trapped on a local minima before converging to the global minimum.

In addition to the original DALM based shaping formulation, the chapter also presented a modification of the fast DALM formulations to an empirical design over multiple positions to achieve spatial robustness to microphone position errors. The empirical design approach was introduced by Jungmann et al. in [39] but their algorithm for finding the shaping filters based on steepest descent is computationally slow. A faster version of this approach based on fast DALM is presented in this chapter.

CHAPTER 6: An approach for joint time and frequency shaping was presented in this chapter. The algorithms presented in Chapter 5 designed shaping filters based in the time domain which can result in undesired peaks and troughs in the frequency spectra of the filtered responses. For effective shaping, both time domain 
shaping and frequency domain flattening of the resultant responses were essential, an approach for which was detailed in Chapter 6 . The frequency spectrum control was implemented as a projection step along with the original DALM iterations. This was possible because the DALM algorithm is a proximal minimization algorithm [35] and this projection step can be easily combined with DALM as an additional projection. An efficient approach for projection onto a low dimensional norm ball was presented in this chapter, which was used to achieve the frequency flatness. The extension of the algorithm to design over multiple microphone positions to achieve spatial robustness was also presented.

The joint time and frequency shaping algorithm presented in this chapter is in the form of a constrained minimization. The frequency projection works by performing the projection for all the positive frequencies one after the other. Therefore, the time shaping would have distorted significantly by the time projection onto all frequencies has completed. This was the reason for a slightly slow convergence of this joint shaping approach; though it was faster than the approaches previously presented in literature $[28,39]$. A better formulation would have been to include a norm of the frequency responses also into the objective function, rather than as a constraint. This was an initial formulation that was tried as a part of this work, but the dual problem in such a case turned out to involve an infimal [150] convolution operator, which was hard to solve. Therefore, the current and simpler projection approach was developed. More details of the earlier formulation involving infimal convolution operators are provided in Section 9.2 for future work.

CHAPTER 7: A statistically robust formulation for achieving spatial robustness in the shaping approach was presented in this chapter. The desired shaping along with spatial robustness was achieved by explicitly limiting the probability of large deviations from the desired performance. This resulted in the formulation of a complicated optimization problem which also required knowledge of the error model of channel variations. Using some assumptions based on the properties of the room response and channel variations, the optimization problem was simplified into a convex SOCP. It was brought out from the simulation studies that this approach was very robust, but had a limitation due to large memory consumption. This may be solvable by using an efficient solver for solving the SOCP; this is left for future work.

CHAPTER 8: An application of the shaping formulation to a real world prob- 
lem of active compensated sound field reproduction was presented in this chapter. A variant of the fast DALM formulation for solving the $\ell_{2}$ regularized $\ell_{2}$ norm minimization was used to solve the impulse response shaping formulation of a pressure matching problem. Application of shaping to AC-SFR provided temperature robustness, which was an issue when using traditional inversion. The added benefit of computational efficiency of DALM suggests that this approach could be very useful for AC-SFR. This application highlights the flexibility and wide applicability of the algorithms developed in this thesis.

\subsection{Future Work}

There is a lot of scope for taking up future work based on this project.

First,the fast DALM algorithms developed in Chapter 5 use a conjugate gradient step in the update equation of dual variable. The computational performance may be further improved if a Preconditioned Conjugate Gradient (PCG) [151] with a proper choice of pre-conditioner is used.

Another option would be to develop an adaptive form of the DALM based shaping algorithm developed in this thesis as outlined in Section 9.1 and detailed here. First, the DALM iterations are performed starting from the initial value till the algorithm converges to the solution. When a channel perturbation occurs, the DALM iterations are resumed from the previous optimum which can then converge to the new minima quicker than performing all DALM iterations from the beginning. Such an adaptive design could be possible because of the convexity of the formulation and the assumption that a channel perturbation just shifts the minimum point; which can be easily reached starting from an intermediate iteration step of the first design.

The experimental validation using the VACS system offer scope for further experimentation. For example, analysis of robustness of the algorithms to change in microphone positions, effect of the presence and absence of a moving object in the room, opening and closing of the door etc. The results can be used to improve the shaping formulations to provide robustness to different kinds of variations.

The frequency flattening step in the joint time and frequency shaping algorithm presented in Chapter 5 was achieved in this thesis using a projection step. 
An alternative would be to include a norm of the frequency response into the original regularized minimization as outlined in Section 9.1. Such a formulation, for example, using $\mathrm{D}-\infty-1$, is given by

$$
\min _{h}\|W C h-W r\|_{\infty}+\lambda\|h\|_{1}+\mu\|G h\|_{\infty}
$$

where $\lambda, \mu$ are the regularization parameters. Let $f_{1}(x)=\|W C h-W r\|_{\infty}, f_{2}(x)=$ $\lambda\|h\|_{1}$ and $f_{3}(x)=\mu\|G h\|_{\infty}$. The dual of this problem is of the form

$$
\max _{y} f_{1}^{*}(y) \square f_{2}^{*}(y) \square f_{3}^{*}(y)
$$

where $\square$ represents the infimal convolution [150] defined as

$$
\left(f_{1} \square f_{2}\right)(y)=\inf \left\{f_{1}(y-z)+f_{2}(z) \mid z \in \mathbb{R}^{n}\right\}
$$

for functions $f_{1}(y)$ and $f_{2}(y)$ defined for $y \in \mathbb{R}^{n}$.

We had an initial attempt at deriving the dual (9.2) for developing a fast DALM algorithm to solve such a problem. However, it turned out to be complicated due to the presence of the infimal convolution operator, which we could not solve. The development of fast DALM to solve such a joint time and frequency shaping problem provides scope for future work.

The statistically robust formulation presented in Chapter 7 used some assumptions of Gaussian IID models for error variations due to microphone positions. This was, however, an approximation; especially the IID assumptions. In an actual scenario, there can be dependencies in the frequency domain. A more accurate model would describe frequency dependence in the channel variances, especially at low frequencies. This opens scope for future work. At present, the statistical model results in slower computation than an equivalent empirical approach based on a design over multiple microphone positions due to the difficulty in finding a fast algorithm for solving an SOCP, since the final optimization problem reduces to an SOCP. But this may change once efficient algorithms for solving SOCPs are developed.

The application of fast DALM algorithms to AC-SFR in Chapter 8 led to achieving temperature robust sound field reproduction in a computationally fast manner. More research into this problem may result in real time implementations of temperature robust AC-SFR systems based on impulse response shaping. This also highlights the flexibility and the applicability of the algorithms developed in this thesis to a wide range of problems, which clearly indicates lot of scope for future work. 


\section{Appendix A}

\section{Preliminaries for Deriving Dual Augmented Lagrangian Method}

This section explains some duality results that simplify the derivation of the Dual Augmented Lagrangian Method (DALM) algorithm.

\section{A.1 Fenchel Duality Theorem}

The Fenchel duality theorem [150] states that if $f$ is a proper convex function on $\mathbb{R}^{N}$ and $g$ is a proper concave function on $\mathbb{R}^{M}$, then

$$
\min _{x} f(x)-g(x)=\max _{y} g_{*}(y)-f^{*}(y)
$$

where $f^{*}$ is the convex conjugate of $f$ and $g_{*}$ is the concave conjugate of $g$, defined as

$$
\begin{array}{ll}
f^{*}(u)=\sup _{x} & \{\langle x, u\rangle-f(x)\} \\
g_{*}(u)=\inf _{x} & \{\langle x, u\rangle-g(x)\}
\end{array}
$$

where $\langle x, u\rangle=x^{\mathrm{T}} u$.

A corollary of the Fenchel duality theorem (corollary 291.2 of theorem 31.2 [150]) is that if $f$ is a proper convex function on $\mathbb{R}^{N}$ and $g$ is a proper concave function on $\mathbb{R}^{M}$ and $A$ is a linear transformation from $\mathbb{R}^{N}$ to $\mathbb{R}^{M}$, then

$$
\min _{x} f(x)-g(A x)=\max _{y} g_{*}(y)-f^{*}\left(A^{T} y\right)
$$




\section{A.1.1 Dual problem of an $\ell_{1}$ regularised objective function}

We can apply the Fenchel duality theorem to a convex minimisation problem of the form

$$
\min _{x} f_{1}(x)+\|x\|_{1}
$$

This is in the form of (A.1) where $g(x)=-f_{1}(x)$ and $f(x)=\|x\|_{1}$. The relationship between convex and concave conjugates of a function $g(x)$ is given by $g_{*}(u)=$ $g^{*}(-u)$ (from equation 3.27 of [152]) and so $g_{*}(y)=-f_{1}^{*}(-y)$. For $f(x)=\|x\|_{1}$ and $x \in \mathbb{R}^{N}$, we have

$$
\begin{aligned}
f^{*}(y) & =\sup _{x}\langle x, y\rangle-\|x\|_{1} \\
& =\sup _{x} \sum_{i=1}^{N} x_{i} y_{i}-\operatorname{sgn}\left(x_{i}\right) x_{i} \\
& =\sup _{x} \sum_{i=1}^{N} x_{i}\left(y_{i}-\operatorname{sgn}\left(x_{i}\right)\right) \\
& = \begin{cases}+\infty & \text { if any } y_{i}>1 \\
0 & \text { otherwise }\end{cases} \\
& =\left\{\begin{array}{ll}
0 & \text { if }\|y\|_{\infty} \leq 1 \\
+\infty & \text { otherwise }
\end{array}=\mathrm{I}_{1}^{\infty}(y)\right.
\end{aligned}
$$

which is the indicator function [41] of the set for which $\ell_{\infty}$ norm $\leq 1$. (The indicator function of a set is zero when the argument is in the set and $+\infty$ otherwise). Using the Fenchel duality theorem, the dual problem for (A.4) can now be written as

$$
\max _{y}-f_{1}^{*}(-y) \quad \text { subject to } y \in \mathrm{B}_{1}^{\infty}
$$

where $\mathrm{B}_{1}^{\infty}$ is the $B_{1}$ ball [34] in which $\|y\|_{\infty} \leq 1$ i.e., $B_{1}^{\infty}$ is defined as

$$
B_{1}^{\infty} \triangleq\left\{y \in \mathbb{R}^{n} \mid\|y\|_{\infty} \leq 1\right\}
$$

When the objective function is in the form of $f_{1}(A x)+\|x\|_{1}$, using (A.3) with $g(x)=-f_{1}(A x)$ and $f(x)=\|x\|_{1}$, the dual becomes

$$
\max _{y}-f_{1}^{*}(-y)-\left\{0 \quad\left\|A^{T} y\right\|_{\infty} \leq 1\right\}
$$


which is equivalent to

$$
\begin{array}{ll}
\max _{y, z} & -f_{1}^{*}(-y)-I_{1}^{\infty}(z) \\
\text { subject to } & z=A^{T} y
\end{array}
$$

Similarly, when the objective is in the form of $f_{1}(A x)+\lambda\|x\|_{1}$, the dual is

$$
\begin{array}{ll}
\max _{y, z} & -f_{1}^{*}(-y)-I_{\lambda}^{\infty}(z) \\
\text { subject to } & z=A^{T} y
\end{array}
$$

where

$$
I_{\lambda}^{\infty}(z)= \begin{cases}0 & \text { if }\|z\|_{\infty} \leq \lambda \\ +\infty & \text { otherwise }\end{cases}
$$

\section{A.1.2 The Fenchel Conjugate of $\|x-b\|_{2}^{2} / 2 \lambda$}

For a function $f_{1}(x)=\|x-b\|_{2}^{2} / 2 \lambda$, the Fenchel conjugate is

$$
f_{1}^{*}(y)=\sup _{x} y^{T} x-\frac{1}{2 \lambda}\|x-b\|_{2}^{2}
$$

The supremum value occurs at any $x$ satisfying

$$
\frac{\partial\left(y^{T} x-\frac{1}{2 \lambda}\|x-b\|_{2}^{2}\right)}{\partial x}=0
$$

This gives

$$
\begin{aligned}
& \lambda y-x+b=0 \\
& \text { or } \quad x=\lambda y+b
\end{aligned}
$$

Substituting (A.14) into (A.12), we obtain

$$
f_{1}^{*}(y)=y^{T}(\lambda y+b)-\frac{\lambda^{2} y^{T} y}{2 \lambda}=y^{T} b+\frac{\lambda y^{T} y}{2}
$$

\section{A.1.3 The Fenchel Conjugate of $\|x-b\|_{\infty}$}

For a function of the form $f_{1}(x)=\|x-b\|_{\infty}$, the conjugate function can be written as

$$
f_{1}^{*}(y)=\sup _{x} y^{T} x-\|x-b\|_{\infty}
$$


Using the transformation $u=x-b$,

$$
\begin{aligned}
f_{1}^{*}(y) & =y^{T} b+\sup _{u} y^{T} u-\|u\|_{\infty} \\
& =y^{T} b+I_{1}^{1}(y)
\end{aligned}
$$

where $I_{1}^{1}(y)$ is the indicator function of $\ell_{1}$ norm. This follows from the basic principle that the Fenchel conjugate of a norm is the indicator function of its dual norm [150]. In general, the norm indicator function $I_{\lambda}^{p}(y)$ is defined as

$$
I_{\lambda}^{p}(y)= \begin{cases}0 & \text { if }\|y\|_{p} \leq \lambda \\ +\infty & \text { otherwise }\end{cases}
$$

\section{A.2 Soft Thresholding function}

A soft thresholding function is defined as

$$
\operatorname{soft}(a, \gamma)= \begin{cases}0 & \text { if }|a|<\gamma \\ a-\gamma & \text { if } a>\gamma \\ a+\gamma & \text { if } a<-\gamma\end{cases}
$$

and this can be used to approximate projection onto an $\ell_{1}$ norm ball. Any minimization problem of the form

$$
\begin{array}{ll}
\min _{y} & \frac{1}{2}\|y-a\|_{2}^{2} \\
\text { subject to } & \|y\|_{1} \leq 1
\end{array}
$$

can be solved iteratively by applying

$$
y_{n}=\operatorname{soft}\left(a_{n}, \gamma\right) \text { for } n=1 \ldots N
$$

to each element $y_{n}$ of $y[112]$ where, $\gamma$ is chosen for each iteration to be

$$
\gamma=\frac{\|a\|_{1}-1}{N}
$$

It can be seen that $\gamma$ quantifies the deviation of the $\ell_{1}$ norm from one in each iteration. 


\section{Appendix B}

\section{Preliminaries for the Statistical Model}

The statistically robust formulation developed in Chapter 7 has been approximated into a convex form using some assumptions based on the properties of room response and some simplifications. Justification for these approximations and simplifications are detailed here.

\section{B.1 Error variance for Independent Identically Distributed Gaussian perturbations}

An important simplification used in the statistically robust formulation is the assumption that the perturbations in the measured channel coefficients (caused by measurement error or sensor movement) are distributed according to an independent and identically distributed Gaussian model. The justification for this assumption is detailed along with the implications in the section.

Consider a single sensor in a pure diffuse reverberant field, perturbed in position by vector $\Delta x$. The sound pressure at a perturbed location $P(x+\Delta x ; \omega)$ is statistically related to the sound pressure at the original location $P(x ; \omega)$ (where $\omega$ is the angular velocity). The correlation in the sound pressure between the two locations can be written:

$$
\frac{E\left\{P^{*}(x+\Delta x ; \omega) P(x ; \omega)\right\}}{\sqrt{E\left\{|P(x+\Delta x ; \omega)|^{2}\right\}} \sqrt{E\left\{|P(x ; \omega)|^{2}\right\}}}=\operatorname{sinc}(k R)
$$


where $R$ is the amplitude of the perturbation and $k$ is the wave number. It has been shown [38] that the average error in perturbing sound pressure due to the perturbation is given by

$$
E\left\{|P(\mathbf{x}+\mathbf{\Delta} \mathbf{x})-P(\mathbf{x})|^{2}\right\}=2 E\left\{|P(\mathbf{x})|^{2}\right\}[1-\operatorname{sinc}(k R)]
$$

Any diffuse sound field can be written [153] as the sum of a number $N_{\mathrm{w}}$ of plane waves $\xi_{n}(\omega) e^{-i k x \cdot \vec{\phi}_{n}}$ :

$$
P(x ; \omega)=\sum_{n=1}^{N_{\mathrm{w}}} \xi_{n}(\omega) e^{-i k x \cdot \vec{\phi}_{n}}
$$

where $\vec{\phi}_{n}$ is a vector of the phase factors. For a small Gaussian perturbation $k R \ll 1$, the complex exponential function can be replaced with its first order expansion. (The first order expansion of an exponential function is specifically $e^{a} \approx(1+a)$.)

$$
P(x+\Delta x ; \omega) \approx \sum_{n=1}^{N_{\mathrm{w}}} \xi_{n}(\omega)\left[1-i k \Delta x \cdot \vec{\phi}_{n}\right] e^{-i k x \cdot \overrightarrow{\phi_{n}}}
$$

The perturbation in sound pressure can thus be written as

$$
P(x+\Delta x ; \omega)-P(x ; \omega)=-i k R \sum_{n=1}^{N_{\mathrm{w}}} \xi_{n}(\omega) e^{-i k x \cdot \vec{\phi}_{n}}\left(\Delta \tilde{x} \cdot \vec{\phi}_{n}\right)
$$

where $\Delta \tilde{x}=\tilde{x} / R$. Gaussian sensor perturbations produce (for small perturbations $k R$ ) Gaussian errors in sound pressure.

In the case of sensor positions distributed in some non-Gaussian manner, a Central Limit theorem [124] would dictate that as $N$ becomes large, the distribution approaches a complex Gaussian distribution. Thence the Gaussian property of the sound field is likely to hold more accurately in sound fields with more multipath.

Typically in 3-D the implicit dimensionality or number of multi-paths required to compose a diffuse sound field [154] is $N \sim(k R+1)^{2}$. In 2-D, number of multi1paths is $N_{\mathrm{w}} \sim 2 k R+1$. Because of these fundamental dimensionalities of sound fields, the Gaussian property in general will hold at higher frequencies and for larger perturbations. 
Generally, the elements in a microphone array are typically not positioned perfectly accurately. Therefore, small Gaussian sensor perturbations in microphone positions produce Gaussian perturbations in the output signal of each microphone. Conversion of these Gaussian perturbations in frequency to the time domain, and then the subsequent combination of these random variables ensures that $d_{k}^{\mathrm{T}} h-r_{k}$ is also Gaussian.

This has been mentioned in the chapter as the conditions under which this assumption will hold i.e., in the cases of either small sensor position perturbation, or strong multi-path.

The frequency variation has been ignored in the above argument, but it is shown in pages 66-69 of [123] that the variance is proportional to frequency. It is valid, to obtain a conservative result, to choose an error variance corresponding to the upper frequency of interest. This will naturally overestimate the errors at low frequencies and we leave it as a future exercise to devise an improved formulation which correctly accounts for this frequency dependence of the error variance.

Given this assumption, equation (7.3) of the chapter can be derived. An illustration of this derivation for a small matrix size is detailed here. Consider a system with number of loudspeakers $L=3$ and number of microphones $M=2$. Let $c_{m l}=\left[c_{m l}(1) c_{m l}(2) c_{m l}(3)\right]$ and $h_{l s}=\left[h_{l s}(1) h_{l s}(2) h_{l s}(3) h_{l s}(4)\right]$. Here, the channel length $N_{c}=3$ and the shaping filter length $N_{h}=4$, therefore the length of the GIR, $N_{r}=N_{c}+N_{h}-1=6$. Using (4.4),

$$
\begin{gathered}
r_{11}=\left[\begin{array}{lll}
C_{11} & C_{12} & C_{13}
\end{array}\right]\left[\begin{array}{l}
h_{11} \\
h_{21} \\
h_{31}
\end{array}\right] \\
C_{11} h_{11}=\left[\begin{array}{cccc}
c_{11}(1) & 0 & 0 & 0 \\
c_{11}(2) & c_{11}(1) & 0 & 0 \\
c_{11}(3) & c_{11}(2) & c_{11}(1) & 0 \\
0 & c_{11}(3) & c_{11}(2) & c_{11}(1) \\
0 & 0 & c_{11}(3) & c_{11}(2) \\
0 & 0 & 0 & c_{11}(3)
\end{array}\right]\left[\begin{array}{c}
h_{11}(1) \\
h_{11}(2) \\
h_{11}(3) \\
h_{11}(4)
\end{array}\right]
\end{gathered}
$$

Assuming all the channel samples to have the same variance $\iota^{2}$ and using $\operatorname{Var}(a X)=$ 
$a^{2} \operatorname{Var}(X)$ where $a$ is a scalar, we have the variance $\sigma_{1}^{2}$ of $C_{11} h_{11}$

$$
\left[\begin{array}{l}
\sigma_{1}^{2}(1) \\
\sigma_{1}^{2}(2) \\
\sigma_{1}^{2}(3) \\
\sigma_{1}^{2}(4) \\
\sigma_{1}^{2}(5) \\
\sigma_{1}^{2}(6)
\end{array}\right]=\left[\begin{array}{c}
\iota^{2} h_{11}^{2}(1) \\
\iota^{2}\left(h_{11}^{2}(1)+h_{11}^{2}(2)\right) \\
\iota^{2}\left(h_{11}^{2}(1)+h_{11}^{2}(2)+h_{11}^{2}(3)\right) \\
\iota^{2}\left(h_{11}^{2}(2)+h_{11}^{2}(3)+h_{11}^{2}(4)\right) \\
\iota^{2}\left(h_{11}^{2}(3)+h_{11}^{2}(4)\right) \\
\iota^{2} h_{11}^{2}(4)
\end{array}\right]
$$

Similarly, variances $\sigma_{2}^{2}$ of $C_{12} h_{21}$ and $\sigma_{3}^{2}$ of $C_{13} h_{31}$ can be computed. Now the variance $\sigma^{2}$ of $r_{11}$ can be computed as $\sigma^{2}=\sigma_{1}^{2}+\sigma_{2}^{2}+\sigma_{3}^{2}$ given by

$$
\left[\begin{array}{c}
\sigma^{2}(1) \\
\sigma^{2}(2) \\
\sigma^{2}(3) \\
\sigma^{2}(4) \\
\sigma^{2}(5) \\
\sigma^{2}(6)
\end{array}\right]=\left[\begin{array}{c}
\iota^{2} \sum_{l=1}^{3} h_{l 1}^{2}(1) \\
\iota^{2} \sum_{l=1}^{3}\left(h_{l 1}^{2}(1)+h_{l 1}^{2}(2)\right) \\
\iota^{2} \sum_{l=1}^{3}\left(h_{l 1}^{2}(1)+h_{l 1}^{2}(2)+h_{l 1}^{2}(3)\right) \\
\iota^{2} \sum_{l=1}^{3}\left(h_{l 1}^{2}(2)+h_{l 1}^{2}(3)+h_{l 1}^{2}(4)\right) \\
\iota^{2} \sum_{l=1}^{3}\left(h_{l 1}^{2}(3)+h_{l 1}^{2}(4)\right) \\
\iota^{2} \sum_{l=1}^{3} h_{l 1}^{2}(4)
\end{array}\right]
$$

which is given by the general equation (7.3) in Chapter 7 .

\section{B.2 Statistically robust model as a Second Order Cone Program}

It has been mentioned in Chapter 7 that the final form of the statistically robust formulation is an SOCP, though it is not in the general form. The expression of this problem (7.13) in the general form is detailed here.

The general form of an SOCP is

$$
\begin{gathered}
\min _{x} \mathbf{f}^{\mathrm{T}} \mathbf{x} \\
\text { subject to } \mathbf{F} \mathbf{x}=\mathbf{g} \\
\left\|\mathbf{A}_{i} \mathbf{x}+\mathbf{b}_{i}\right\|_{2} \leq \mathbf{c}_{i}^{\mathrm{T}} \mathbf{x}+\mathbf{d}_{i} i=1, \ldots m
\end{gathered}
$$


Our final problem is

$$
\begin{array}{cl}
\min _{\gamma, \mathbf{h}} & \gamma \\
\text { subject to } \mathbf{B h}=\mathbf{1} & \\
\gamma+\left(\mathbf{c}_{k}^{\mathrm{T}} \mathbf{h}-r_{k}\right) \geq \sqrt{2} \sigma_{k} \operatorname{erfi}\left(2-2 \mathrm{P}_{0, \max } / w_{\text {early }}\right), & k \in \mathbb{A}_{3} \\
\gamma+\left(\mathbf{c}_{k}^{\mathrm{T}} \mathbf{h}-r_{k}\right) \geq \sqrt{2} \sigma_{k} \operatorname{erfi}\left(1-\mathrm{P}_{0, \text { max }} / w_{\text {late }}\right), & k \in \mathbb{A}_{4} \\
\gamma+\left(\mathbf{c}_{k}^{\mathrm{T}} \mathbf{h}-r_{k}\right) \geq \sqrt{2} \sigma_{k} \operatorname{erfi}\left(1-\mathrm{P}_{0, \text { max }} / w_{\text {pre-echo }}\right), & k \in \mathbb{A}_{1} \\
\gamma+\left(\mathbf{c}_{k}^{\mathrm{T}} \mathbf{h}-r_{k}\right) \geq \sqrt{2} \sigma_{k} \text { erfi }\left(1-\mathrm{P}_{0, \text { max }} / w_{\text {crosstalk }}\right), & k \in \mathbb{A}_{5}
\end{array}
$$

In the final problem (7.13), let $\mathbf{x}=\left[\begin{array}{ll}\gamma & \mathbf{h}^{\mathrm{T}}\end{array}\right]^{\mathrm{T}}$ and $\mathbf{f}=\left[\begin{array}{ll}1 & \mathbf{0}_{L \times M \times N_{\mathrm{h}}}^{\mathrm{T}}\end{array}\right]^{\mathrm{T}}$.

The objective function can be written as

$$
\gamma=\mathbf{f}^{\mathrm{T}} \mathbf{x}
$$

Let the matrix $\mathbf{E}=\left[\begin{array}{ll}\mathbf{0} & \mathbf{B}\end{array}\right]$. Then the equality constraint in the final problem (7.13) can be written as

$$
\mathrm{Ex}=1
$$

In the RHS of the inequality constraints, $\sqrt{2} \iota^{2} \operatorname{erfi}(1-\mathbf{P} / \mathbf{w})$ is a constant vector K.

$\sum_{j} \mathbf{h}_{j}^{2}$ can be represented in the form $\mathbf{h}^{\mathrm{T}} \mathbf{G}_{i} \mathbf{h}$ where matrix $G_{i}$ selects the corresponding elements of $\mathbf{h}$ needed for the summation $\sum_{j} \mathbf{h}_{j}^{2}$. Therefore, $\sum_{j} \mathbf{h}_{j}^{2}$ can be expressed as $\mathbf{x}^{\mathrm{T}} \mathbf{F}_{i} \mathbf{x}$ where $F_{i}=\left[\begin{array}{ll}\mathbf{0} & G_{i}\end{array}\right]$. Also, let $\mathbf{u}_{i}=\left[\begin{array}{ll}1 & \mathbf{c}_{\mathbf{k}}^{\mathrm{T}}\end{array}\right]^{\mathrm{T}}$, $\mathbf{v}_{i}=\left[\begin{array}{ll}0 & -\mathbf{r}_{\mathbf{k}}^{\mathrm{T}}\end{array}\right]^{\mathrm{T}}$.

The inequality equations can now be written in the form

$$
\mathbf{x}^{\mathrm{T}} \mathbf{F}_{i} \mathbf{x}-\frac{1}{K_{i}} \mathbf{u}_{i}^{\mathrm{T}} \mathbf{x}+\frac{1}{K_{i}} \mathbf{v}_{i} \leq 0
$$

By completion of squares and taking square root on both sides, the inequality equations can be expressed as

$$
\left\|\mathbf{F}_{i}^{1 / 2} \mathbf{x}+\mathbf{F}_{i}^{-1 / 2} \frac{\mathbf{u}_{i}}{2 K_{i}}\right\|_{2} \leq\left(\frac{\mathbf{u}_{i}}{2 K_{i}} \mathbf{F}_{i}^{-1} \frac{\mathbf{u}_{i}}{2 K_{i}}-\frac{1}{K_{i}} \mathbf{v}_{i}\right)^{1 / 2}
$$

It can be seen that the RHS of the above inequality equation (B.12) is just a constant vector $\mathbf{d}_{i}$. Similarly the second term in LHS is also a constant vector $\mathbf{b}_{i}$. 
Therefore, (B.12) can be written as

$$
\left\|\mathbf{F}_{i}^{1 / 2} \mathbf{x}+\mathbf{b}_{i}\right\|_{2} \leq \mathbf{d}_{i}
$$

. Now the final statistically robust problem (7.13) can be written using (B.10), (B.11) and (B.13) as

$$
\begin{gathered}
\min _{x} \mathbf{f}^{\mathrm{T}} \mathbf{x} \\
\text { subject to } \mathbf{E x}=\mathbf{1} \\
\left\|\mathbf{F}_{i}^{1 / 2} \mathbf{x}+\mathbf{b}_{i}\right\|_{2} \leq \mathbf{d}_{i} i=1, \ldots L * M * N_{\mathrm{r}}
\end{gathered}
$$

which is clearly an SOCP in the form of (B.9). 


\section{List of Abbreviations}

\begin{tabular}{|l|l|}
\hline Acronym & \multicolumn{1}{|c|}{ Expansion } \\
\hline AC-SFR & Active Compensated Sound Field Reproduction \\
BPDN & Basis Pursuit DeNoising \\
CCF & Cross-talk Cancelation Filters \\
CDF & Cumulative Density Function \\
CDR & Cross-talk to Direct response Ratio \\
CG & Conjugate Gradient \\
CVX Solver & Convex Solver \\
DALM & Dual Augmented Lagrangian Method \\
DFT & Discrete Fourier Transform \\
DRR & Direct to Reverberation energy Ratio \\
FAO & Forward Adjoint Oracle \\
FFT & Fast Fourier Transform \\
GIR & Global Impulse Response \\
HRTF & Head Related Transfer Function \\
IID & Independent Identically Distributed \\
IRS & Impulse Response Shaping \\
LS & Least Squares \\
MSE & Mean Square Error \\
MINT & Multiple input/output INverse Theorem \\
nPRQ & Perceived Reverberation Quality \\
NIR & Nett Impulse Response \\
PDF & Probability Density Function \\
P-MINT & Partial-Multiple input/output INverse Theorem \\
PeCCS & Perceptually enhanced Constrained Channel Shortening \\
QCQP & Quadratically Constrained Quadratic Programming \\
RMCLS & Relaxed Multi-channel Least Squares \\
RTF & Room Transfer Function \\
\hline
\end{tabular}




\begin{tabular}{|l|l|}
\hline RIR & Room Impulse Response \\
SD & Steepest Descent \\
SFM & Spectral Flatness Measure \\
SOCP & Second Order Conic Program \\
VACS & Validation of Acoustic Channel Shortening \\
\hline
\end{tabular}




\section{Bibliography}

[1] F. Rumsey and T. McCormik, Sound and recording. Focal Press, Taylor and Francis, 6 ed., 2013.

[2] S. Parker, ed., Acoustics Source Book. Mcgraw-Hill, Blacklick, Ohio, U.S.A, 1 ed., 1988.

[3] J. O. Pickles, An Introduction to the Physiology of Hearing. London Academic Press, 1982.

[4] H. Kuttruff, Room Acoustics. Spon Press, Taylor \& Francis, 5 ed., 2009.

[5] G. Ginolhac and G. Jourdain, "Detection in presence of reverberation," in Proc. MTS/IEEE Conf. ES Exhb. OCEANS 2000, vol. 2, pp. 1043-1046, 2000.

[6] "Acoustics demystified: creating better - sounding rooms is easier than you think," Feb 2016.

[7] E. Thompson, The sound scope of modernity-architectural acoustics and the culture of listening in America (1900-1933). MIT press, 2002.

[8] J. Walker, The Flying Circus of Physics (With answers). John Wiley \& Sons, 1975.

[9] J. M. Pickett, "Effects of vocal force on the intelligibility of speech sounds," J. Acoust. Soc. Am., vol. 28, no. 5, pp. 902--901, 1956.

[10] M. A. Picheny, N. I. Durlach, and L. D. Braida, "Speaking clearly for the hard of hearing i: Intelligibility differences between clear and conversational speech," J. Speech \&6 Hearing Res., vol. 28, no. 1, pp. 96-103, 1985.

[11] M. A. Picheny, N. I. Durlach, and L. D. Braida, "Speaking clearly for the hard of hearing. ii: Acoustic characteristics of clear and conversational speech," J. Speech \& Hearing Res., vol. 29, no. 4, pp. 434-446, 1986. 
[12] M. L. G. Lecumberri, M. Cooke, and A. Cutler, "Non-native speech perception in adverse conditions: A review," J. Speech Communication, vol. 52, pp. 864-886, 2010.

[13] J. S. Bradley and H. Sato, "On the importance of early reflections for speech in rooms," J. Acoust. Soc. Am., vol. 113, pp. 3233-3244, Jun 2003.

[14] I. Kodrasi, S. Goetze, and S. Doclo, "A perceptually constrained channel shortening technique for speech dereverberation," in Proc. IEEE Int. Conf. Acoust., Speech \& Signal Processing, pp. 151-155, May 2013.

[15] T. Holman, Surround Sound, up and running. Focal Press, Taylor and Francis, 2 ed., 2008.

[16] S. Spors, H. Buchner, R. Rabenstein, and W. Herbordt, "Adaptive listening room compensation for spatial audio systems," in Proc. IEEE 12th European Signal Processing Conf., pp. 1381-1384, Sep 2004.

[17] S. Spors, H. Buchner, R. Rabenstein, and W. Herbordt, "Active listening room compensation for massive multichannel sound reproduction systems using wave-domain adaptive filtering," J. Acoust. Soc. Am., vol. 122, pp. 354369, Jul 2007.

[18] M. Miyoshi and Y. Kaneda, "Inverse filtering of room acoustics," IEEE Trans. Acoust., Speech \& Signal Processing, vol. 36, pp. 145-152, Feb 1988.

[19] M. Kallinger and A. Mertins, "Room impulse response shortening by channel shortening concepts," in IEEE Asilomar Conf., pp. 898-902, 2005.

[20] T. Mei, A. Mertins, and M. Kallinger, "Room impulse response shortening with infinity-norm optimization," in Proc. IEEE Int. Conf. Acoust., Speech E) Signal Processing, pp. 3745-3748, 2009.

[21] A. Mertins, T. Mei, and M. Kallinger, "Room impulse response shortening/reshaping with infinity- and pnorm optimization," IEEE Trans. Audio, Speech \&f Language Processing, vol. 18, no. 2, pp. 249-259, 2010.

[22] M. R. P. Thomas, N. D. Gaubitch, and P. A. Naylor, "Application of channel shortening to acoustic channel equalization in the presence of noise and estimation error," in Proc. IEEE Wrkshp. Applcns. Signal Processing to Audio E6 Acoust., pp. 113-116, Oct 2011. 
[23] P. J. W. Melsa, R. C. Younce, and C. E. Rohrs, "Impulse Response Shortening for Discrete Multitone Transceivers," IEEE Trans. Comm., vol. 44, no. 12 , pp. 1662-1672, 1996.

[24] G. Arslan, B. L. Evans, and S. Kiaei, "Equalization for discrete multitone transceivers to maximize bit rate," IEEE Trans. Signal Processing, vol. 49, no. 12, pp. 3123-3135, 2001.

[25] D. Wübben and K.-D. Kammeyer, "Impulse shortening and equalization of frequency-selective MIMO channels with respect to layered space-time architectures," Elsevier J. Signal Processing, vol. 83, pp. 1643-1659, Aug 2003.

[26] I. Barhumi, G. Leus, and M. Moonen, "Time-domain channel shortening and equalization of OFDM over doubly-selective channels," Proc. IEEE Int. Conf. Acoust., Speech 65 Signal Processing, vol. 3, pp. 801-804, 2004.

[27] T. Betlehem, P. D. Teal, and Y. Hioka, "Efficient crosstalk canceler design with impulse response shortening filters," in Proc. IEEE Int. Conf. Acoust., Speech 85 Signal Processing, pp. 393-396, Mar 2012.

[28] J. O. Jungmann, R. Mazur, M. Kallinger, T. Mei, and A. Mertins, "Combined acoustic mimo channel crosstalk cancellation and room impulse response reshaping," IEEE Trans. Audio, Speech \& Language Processing, vol. 20, pp. 1829-1842, Aug 2012.

[29] M. Kallinger and A. Mertins, "Impulse response shortening for acoustic listening room compensation," in Proc. Int. Wrkshp. Acoust. Echo $\&$ Noise Control, pp. 197-200, 2005.

[30] W. C. Sabine, Collected papers on acoustics. Harvard University Press, 1922.

[31] H. I. K. Rao, J. V. Mathews, and Y. C. Park, "A minimax approach for the joint design of acoustic crosstalk cancellation filters," IEEE Trans. Audio, Speech \& Language Processing, vol. 15, pp. 2287-2298, Nov 2007.

[32] R. Mazur, J. O. Jungmann, and A. Mertins, "Optimized gradient calculation for room impulse response reshaping algorithm based on p-norm optimization," in Proc. IEEE Int. Conf. Acoust., Speech 65 Signal Processing, pp. 185-188, Mar 2012. 
[33] P. C. Hansen, "The L-curve and its use in the numerical treatment of inverse problems," J. Computational Inverse Prob. Electrocardiology, no. 5, pp. 119$142,2001$.

[34] A. Yang, A. Ganesh, Z. Zhou, S. Sastry, and Y. Ma, "Fast $\ell_{1}$ minimisation algorithms and an application in robust face recognition: a review," technical report ucb/eecs-2010-13, EECS Department, University of California, Berkeley, Feb 2010.

[35] R. Tomioka, T. Suzuki, and M. Sugiyama, "Super-linear convergence of Dual Augmented Lagrangian Algorithm for sparsity regularized estimation," J. Machine Learning Res., vol. 12, pp. 1537-1586, May 2011.

[36] P. E. Gill and D. P. Robinson, "A primaldual augmented lagrangian," J. Computational Optimization \& Applcns., vol. 51, Jan 2012.

[37] D. L. Donoho, "Compressed sensing," IEEE Trans. Information Theory, vol. 52, pp. 1289-1306, April 2006.

[38] B. D. Radlovic̀, R. C. Williamson, and R. A. Kennedy, "Equalization in an acoustic reverberant environment: robustness results," IEEE Trans. Speech E3 Audio Processing, vol. 8, pp. 311-319, May 2000.

[39] J. O. Jungmann, R. Mazur, and A. Mertins, "Perturbation of room impulse responses and its application in robust listening room compensation," in Proc. IEEE Int. Conf. Acoust., Speech \& Signal Processing, pp. 433-437, May 2013.

[40] S. Singh, P. D. Teal, P. A. Dmochowski, and A. J. Coulson, "Robust cognitive radio cooperative beamforming," IEEE Trans. Wireless Communications, vol. 13, Nov 2013.

[41] S. Boyd and L. Vanderberghe, Convex Optimization. Cambridge University Press, 2004.

[42] H. Nelisse and J. Nicholas, "Characterisation of a diffuse field in a reverberant room," J. Acoust. Soc. Am., vol. 101, pp. 3517-3524, Jun 1997.

[43] M. R. Schroeder and K. H. Kuttruff, "On frequency response curves in rooms. comparison of experimental, theoretical, and monte carlo results for the average frequency spacing between maxima," J. Acoust. Soc. Am., vol. 34, no. 1, pp. 76-80, 1962. 
[44] M. Hodgson, "When is diffuse-field theory applicable?," Elsevier J. Applied Acoust., vol. 49, pp. 197-207, Nov 1996.

[45] J. B. Allen and D. A. Berkley, "Image method for efficiently simulating small-room acoustics," J. Acoust. Soc. Am., vol. 65, Apr 1979.

[46] D. B. Ward and T. D. Abhayapala, "Reproduction of a plane-wave sound field using an array of loudspeakers," IEEE Trans. Speech 86 Audio Processing, vol. 9, Sep 2001.

[47] M. A. Gerzon, "Ambisonics in multichannel broadcasting and video," J. Audio Eng. Soc, vol. 33, no. 11, pp. 859-871, 1985.

[48] J. S. Bamford and J. Vanderkooy, "Ambisonic sound for us," in Proc. 99th Audio Eng. Soc. Conv., Oct 1995.

[49] M. A. Poletti, "The design of encoding functions for stereophonic and polyphonic sound systems," J. Audio Eng. Soc, vol. 44, no. 11, pp. 948-963, 1996.

[50] M. Poletti, "Robust two-dimensional surround sound reproduction for nonuniform loudspeaker layouts," J. Audio Eng. Soc, vol. 55, no. 7/8, pp. 598-610, 2007.

[51] M. M. Boone, E. N. G. Verheijen, and P. F. van Tol, "Spatial sound field reproduction by wave field synthesis," J. Audio Eng. Soc, vol. 43, no. 12, pp. 1003-1012, 1995.

[52] P. A. Nelson, H. Hamada, and S. J. Elliott, "Adaptive inverse filters for stereophonic sound reproduction," IEEE Trans. Signal Processing, vol. 40, pp. 1621-1632, Jul 1992.

[53] D. G. Malham and A. Myatt, "3-D sound spatialization using ambisonic techniques," J. Computer Music, vol. 19, no. 4, pp. 58-70, 1995.

[54] M. A. Poletti, "Three-dimensional surround sound systems based on spherical harmonics," J. Audio Eng. Soc, vol. 53, no. 11, pp. 1004-1025, 2005.

[55] M. Johansson, L. J. Brännmark, A. Bahne, and M. Sternad, "Sound field control using a limited number of loudspeakers," in Proc.36th AES Int. Conf. Automotive Audio, Jun 2009. 
[56] L.-J. Brännmark, A. Bahne, and A. Ahlén, "Improved loudspeaker-room equalization using multiple loudspeakers and MIMO feedforward control," in Proc. IEEE Int. Conf. Acoust., Speech E Signal Processing, pp. 237-240, 2012.

[57] L. J. Brännmark, A. Bahne, and A. Ahlén, "Compensation of loudspeakerroom responses in a robust MIMO control framework," IEEE Trans. Audio, Speech \& Language Processing, vol. 21, pp. 1201-1216, June 2013.

[58] T. Betlehem and P. D. Teal, "A constrained optimization approach for multizone surround sound," in Proc. IEEE Int. Conf. Acoust., Speech 8 Signal Processing, pp. 437-440, 2011.

[59] S. J. Elliot and M. Jones, "An active headrest for personal audio," J. Acoust. Soc. Am., vol. 119, May 2006.

[60] J. Y. Park, J. H. Chang, and Y. H. Kim, "Generation of independent bright zones for a two-channel private audio system," J. Audio Eng. Soc., vol. 58, no. 5, pp. 382-393, 2010.

[61] Y. Kim, S. Ko, J. W. Choi, and J. Kim, "Optimal filtering for focussed sound field reproductions using a loudspeaker array," in Proc. AES 126th Conv., May 2009.

[62] Y. Hioka, K. Niwa, S. Sakauchi, K. Furuya, and Y. Haneda, "Evaluating estimation of direct-to-reverberation energy ratio using $\mathrm{d} / \mathrm{r}$ spatial correlation matrix model," in Proc. Int. Conf. Acoust., 2010.

[63] N. A. Gumerov and R. Duraiswami, "Modeling the effect of a nearby boundary on the hrtf," in Proc. IEEE Int. Conf. Acoust., Speech 8 Signal Processing, vol. 5, pp. 3337-3340, 2001.

[64] V. R. Alghazi, R. O. Duda, R. Duraiswami, N. A. Gumerov, and Z. Tang, "Approximating the head-related transfer function using the simple geometric models of head and torso," J. Acoust. Soc. Am., vol. 112, pp. 2053-2064, Nov 2002.

[65] W. Zhang, E. A. P. Habets, and P. A. Naylor, "On the use of channel shortening in multichannel acoustic system equalization," in Proc. Int. Wrkshp. Acoust. Echo \& Noise Control, vol. 1, Aug 2010. 
[66] I. Kodrasi and S. Doclo, "Robust partial multichannel equalization techniques for speech dereverberation," in Proc. IEEE Int. Conf. Acoust. Speech 8 Signal Processing, pp. 537-540, 2012.

[67] I. Kodrasi, S. Goetze, and S. Doclo, "Increasing the robustness of acoustic multichannel equalizatoin by means of regularization," in Proc. Int. Wrkshp. Acoust. Signal Enhancement, pp. 4-6, Sep 2012.

[68] I. Kodrasi, S. Goetze, and S. Doclo, "Regularization for partial multichannel equalization for speech dereverberation," IEEE Trans. Audio, Speech 8 Language Processing, vol. 21, no. 9, pp. 1879-1890, 2013.

[69] S. J. Elliott and M. Jones, "An active headrest for personal audio," J. Acoust. Soc. Am., vol. 119, no. 5, pp. 2702-2709, 2006.

[70] J. Cheer, S. J. Elliott, and M. F. S. Galvez, "Design and implementation of a car cabin personal audio system," J. Audio Eng. Soc., vol. 61, pp. 412-424, Jun 2013.

[71] T. Mei, A. Mertins, and M. Kallinger, "Room impulse response reshaping/shortening based on least mean squares optimizationwith infinity norm constraint," in 16th Int. Conf. Digital Signal Processing, pp. 1-6, Jul 2009.

[72] F. Lim and P. A. Naylor, "Relaxed multichannel least squares with constrained initial taps for multichannel dereverberation," in Proc. Int. Wrskhp. Acoust. Signal Enhancement, pp. 4-6, Sep 2012.

[73] F. Lim, M. R. P. Thomas, and P. A. Naylor, "Mintformer: a spatially aware equalizer," in IEEE Wrkshp. Applcns. Signal Processing to Audio 8 Acoust., 2013.

[74] P. C. Hansen and D. O'Leary, "The use of the L-curve in the regularization of discrete illposed problems," SIAM J. Scientific Computing, vol. 14, pp. 14871503, 1993.

[75] J. M. Buchholz, J. Mourjopoulos, and J. Blauert, "Room masking: Understanding and modelling the masking of room reflections," in Proc. 110th Conv. Audio Eng. Soc., 2001.

[76] J. O. Jungmann, R. Mazur, M. Kallinger, and A. Mertins, "Robust combined crosstalk cancellation and listening-room compensation," in Proc. IEEE 
Wrkshp. Applcn. Signal Processing to Audio and Acoust., vol. 22, pp. 9-12, 2011.

[77] L. D. Fielder, "Practical limits for room equalization," in Proc. 111th Conv. Audio Eng. Soc., no. 5481, Nov 2001.

[78] M. Kahrs and K. E. Brandenburg, eds., Applications of Digital Signal Processing to Audio and Acoustics. Springer, 2002.

[79] T. Hikichi, M. Delcroix, and M. Miyoshi, "Inverse filtering for speech dereverberation less sensitive to noise and room transfer function fluctuations," Eurasip J. Advances in Signal Processing, Feb 2007.

[80] J. O. Jungmann, T. Mei, S. Goetze, and A. Mertins, "Room impulse response reshaping by joint optimization of multiple p-norm based criteria," in Proc. EUSIPCO, pp. 1-5, 2011.

[81] L. J. Brännmark, "Robust audio precompensation with probabilistic modeling of transfer function variability," in Proc. IEEE Wrkshp. Applns. Signal Processing to Audio \& Acoust., pp. 193-196, Oct 2009.

[82] A. Bahne, L. J. Brännmark, and A. Ahlén, "Symmetric loudspeaker-room equalization utilizing a pairwise channel similarity criterion," IEEE Trans. Signal Processing, vol. 61, pp. 6276-6290, Dec 2013.

[83] A. Barkefors, M. Sternad, and L. J. Brännmark, "Design and analysis of linear quadratic gaussian feedforward controllers for active noise control," IEEE/ACM Trans. Audio, Speech \& Language Processing, vol. 22, pp. 17771791, Dec 2014.

[84] N. D. Gaubitch and P. A. Naylor, "Equalization of multichannel acoustic systems in oversampled subbands," IEEE Trans. Audio, Speech $\&$ Language Processing, vol. 17, pp. 1061-1070, Aug 2009.

[85] A. Cauchy, "Méthode générale pour la résolution des systèms d'équations simultanées," pp. 536-538, 1847.

[86] M. J. Box, D. Davies, and W. H. Swann, Non-Linear optimisation Techniques. Oliver and Boyd, 1969.

[87] L. Armijo, "Minimization of functions having Lipschitz continuous first partial derivatives," Pacific J. Math., vol. 16, no. 1, pp. 1-3, 1966. 
[88] J. Barzilai and J. M. Borwein, "Two point step size gradient methods," IMA J. Numerical Analysis, vol. 8, pp. 141-148, 1988.

[89] Y. H. Dai and R. Fletcher, "On the asymptotic behaviour of some new gradient methods," tech. rep., Dept. of Math. University of Dundee, Scotland, 2003.

[90] Y. H. Dai, J. Y. Yuan, and Y. Yuan, "Modified two-point step-size gradient methods for unconstrained optimization," Computational Optimization 8 Applcns., vol. 22, pp. 103-109, 2002.

[91] Y. H. Dai and Y. Yuan, "Alternate minimization gradient method," IMA J. Numerical Analysis, vol. 23, pp. 377-393, 2003.

[92] S. Mehrotra, "On the implementation of a primal-dual interior point method," SIAM J. Optimization, vol. 2, no. 4, pp. 575-601, 1992.

[93] M. R. Hestenes and E. Stiefel, "Methods of conjugate gradients for solving linear systems," J. Res. National Bureau of Stds., vol. 49, Dec 1952.

[94] Y. Saad, Iterative methods for sparse linear systems. SIAM, 2 ed., 2004.

[95] J. A. Tropp, A. C. Gilbert, and M. Strauss, "Algorithms for simultaneous sparse approximations ; part i : greedy pursuit," J. Signal Proc. - Sparse approximations in signal and image processing, vol. 86, pp. 572-588, 2006.

[96] J. A. Tropp and A. C. Gilbert, "Signal recovery from random measurements via orthogonal matching pursuit," IEEE Trans. Information Theory, vol. 53, Dec 2007.

[97] D. L. Donoho, Y. Tsaig, I. Drori, and J. L. Starck, "Sparse solution of underdetermined systems of linear equations by stagewise orthogonal matching pursuit," J. Computational Optimization \& Applcns., vol. 58, Feb 2012.

[98] I. W. Selesnik, "Sparse signal restoration," Apr 2010.

[99] A. Beck and M. Teboulle, "A fast iterative shrinkage-thresholding algorithm for linear inverse problems," SIAM J. Imaging Sciences, vol. 2, no. 1, pp. 183-202, 2009. 
[100] I. Daubachies, M. Defriese, and C. D. Mol, "An iterative thresholding algorithm for linear inverse problems with sparsity constraints," J.Fourier Analysis \& Applcns., vol. 14, pp. 183-202, Dec 2008.

[101] M. A. T. Figueiredo, J. M. BioucasDias, and R. D. Nowak, "Majorization minimization algorithms for wavelet-based image restoration," IEEE Trans. Image Processing, vol. 16, pp. 2980-2991, Dec 2007.

[102] P. E. Gill and D. P. Robinson, "A primal-dual augmented lagrangian," J. Computational Optimization \& Applcns., vol. 51, pp. 1-25, Jan 2012.

[103] S. F. Cotter and B. D. Rao, "Sparse channel estimation via matching pursuit with application to equalization," IEEE Trans. Communications, vol. 50, pp. 374-377, Mar 2002.

[104] H. H. Bauschke and Y. Lucet, "What is a Fenchel conjugate?," in Notices of the AMS, 2012.

[105] M. R. Hestenes and E. Stiefel, "Methods of conjugate gradients for solving linear systems," J. Res. National Bureau of Stds., vol. 49, no. 6, 1952.

[106] J. Makhoul and J. Wolf, "Linear prediction and the spectral analysis of speech," Bolt, Beranek and Newman 2304, Advanced Research Projects Agency, Arlington, Virginia, Aug 1972.

[107] J. Johnston, "Transform coding of audio signals using perceptual noise criteria," IEEE J. Selected Areas in Comm., vol. 6, pp. 314--323, Feb 1988.

[108] S. Diamond and S. Boyd, "Matrix-free convex optimization modeling," arXiv preprint arXiv:1506.00760 (2015), Nov 2015.

[109] L. Krishnan, P. D. Teal, and T. Betlehem, "Fast algorithms for acoustic impulse response shaping," IEEE Trans. Audio, Speech 8 Language Processing (submitted for publication), 2017.

[110] L. Krishnan, P. D. Teal, and T. Betlehem, "A sparsity based approach for acoustic room impulse response shortening," in Proc. IEEE Statistical Signal Processing Wrkshp., pp. 284-287, Jun 2014.

[111] L. Krishnan, P. D. Teal, and T. Betlehem, "A robust sparse approach to acoustic impulse response shaping," in Proc. IEEE Int. Conf. Acoust., Speech E Signal Processing, pp. 738-742, Apr 2015. 
[112] J. Songsiri, "Projection onto an $\ell_{1}$ norm ball with application to identification of sparse autoregressive models," in Proc. Asean Symp. Automatic Control, 2011.

[113] D. L. Donoho and I. M. Johnstone, "Adapting to unknown smoothness via wavelet shrinkage," J. Am. Statistical Assoc., vol. 90, no. 432, pp. 1200-1224, 1995 .

[114] "Datasheet of HDSPe MADI FX."

[115] "Datasheet of Micstasy pre-amplifiers."

[116] P. D. Teal, L. Krishnan, and T. Betlehem, "An efficient projection onto a low-dimensional $\ell_{2}$ norm ball," J. Engg. Opt. (submitted for publication), 2017.

[117] D. C. Youla and H. Webb, "Image restoration by the method of convex projections: part 1 theory," IEEE Trans. Medical Imaging, vol. 1, pp. 81-94, Oct 1982 .

[118] P. L. Combettes, "The foundations of set theoretic estimation," IEEE Proc., vol. 81, Feb 1993.

[119] E. L. Rees, "Graphical discussion of the roots of a quartic equation," The Am. Math. Monthly, vol. 29, no. 2, pp. 51—-55, 1922.

[120] J. O. Jungmann, R. Mazur, and A. Mertins, "Joint time-domain reshaping and frequency-domain equalization of room impulse responses," in Proc. IEEE Int. Conf. Acoust., Speech \& Signal Processing, pp. 6642-6646, May 2014.

[121] J. O. Jungmann, R. Mazur, and A. Mertins, "Joint time- and frequencydomain reshaping of room impulse responses," in Proc. IEEE Int. Conf. Acoust., Speech 6 Signal Processing, pp. 733-737, Apr 2015.

[122] L. Krishnan, T. Betlehem, and P. D. Teal, "A statistically robust approach to acoustic impulse response shaping," IEEE Signal Processing Letters, vol. 24, pp. 1138-1142, Aug 2017.

[123] H. L. Van Trees, Optimum Array Processing (Part 1V: Detection, Estimation and Modulation Theory. Wiley- Interscience, 2002. 
[124] A. Papoulis and S. U. Pillai, Probability, Random Variables and Stochastic Processes. McGraw-Hill Higher Education, 4 ed., 2002.

[125] F. C. Leone, L. S. Nelson, and R. B. Nottingham, "The folded normal distribution," Technometrics, vol. 3, no. 4, pp. 543-550, 1961.

[126] "CVX: Matlab software for disciplined convex programming," June 2015.

[127] K. Zhong, P. Jain, and A. Kapoor, "Fast second-order cone programming for safe mission planning," CoRR, vol. abs/1609.05243, 2016.

[128] W. Zhang and T. D. Abhayapala, "Three dimensional sound field reproduction using multiple circular loudspeaker arrays: functional analysis guided approach," IEEE/ACM Trans. Audio, Speech \& Language Processing, vol. 22, pp. 1184-1194, Jul 2014.

[129] M. A. Poletti, T. Betlehem, and T. D. Abhayapala, "Higher-order loudspeakers and active compensation for improved 2D sound field reproduction in rooms," J. Audio Eng. Soc, vol. 63, no. 1/2, pp. 31-45, 2015.

[130] S. Petrausch, S. Spors, and R. Rabenstein, "Simulation and visualization of room compensation for wave field synthesis with the functional transformation method," in Proc. 119th Conv. Audio Eng. Soc., 2005.

[131] P.-A. Gauthier and A. Berry, "Adaptive wave field synthesis with independent radiation mode control for active sound reproduction: theory," $J$. Acoust. Soc. Am., vol. 119, no. 5, pp. 2721-2737, 2006.

[132] P.-A. Gauthier and A. Berry, "Adaptive wave field synthesis for sound field reproduction: theory, experiments, and future perspectives," J. Audio Eng. Soc., vol. 55, no. 12, pp. 1107-1124, 2007.

[133] P.-A. Gauthier and A. Berry, "Adaptive wave field synthesis for active sound field reproduction: experimental results," J. Acoust. Soc. Am., vol. 123, no. 4, pp. 1991-2002, 2008.

[134] M. Schneider and W. Kellermann, "A wave-domain model for acoustic MIMO systems with reduced complexity," in Proc. IEEE HSCMA, (Edinburgh, UK), pp. 133-138, May 2011. 
[135] D. Talagala, W. Zhang, and T. Abhayapala, "Efficient multi-channel adaptive room compensation for spatial sound field reproduction using a modal decomposition," IEEE/ACM Trans. Audio, Speech \& Language Processing, vol. 22, pp. 1522--1532, Oct 2014.

[136] M. A. Poletti and T. D. Abhayapala, "Spatial sound reproduction systems using higher order loudspeakers," in Proc. IEEE Int. Conf. Acoust., Speech E Signal Processing, pp. 57-60, May 2011.

[137] M. A. Poletti, F. M. Fazi, and P. A. Nelson, "Sound reproduction systems using variable-directivity loudspeakers," J. Acoust. Soc. Am., vol. 129, pp. 1429-1438, Mar 2011.

[138] M. A. Poletti, T. D. Abhayapala, and P. Samarasinghe, "Interior and exterior sound field control using general two dimensional higher-order variabledirectivity sources," J. Acoust. Soc. Am., vol. 129, no. 5, pp. 3814-3823, 2012.

[139] M. A. Poletti and T. Betlehem, "Design of a prototype variable directivity loudspeaker for improved surround sound reproduction in rooms," in Proc. 52nd Int. Conf. Sound Field Control - Eng. \&5 Perception, Sep 2013.

[140] T. Betlehem, C. Anderson, and M. A. Poletti, "A directional loudspeaker array for surround sound in reverberant rooms," in Proc. Int. Conf. Acoust., (Sydney), 2010.

[141] A. Carini, S. Cecchi, and L. Romoli, "Multipoint room response equalization with group delay compensation," in Proc. Int. Wrkshp. Acoust. Echo $\&$ Noise Control, Aug 2010.

[142] L. Bianch, F. Antonacci, A. Canclini, A. Sarti, and S. Tubaro, "A psychoacoustic-based analysis of the impact of pre-echoes and post-echoes in soundfield rendering applications," in Proc. Int. Wrkshp Acoustic Echo 8 Noise Control (IWAENC), (Aachen), Sep 2012.

[143] T. Hikichi and F. Itakura, "Time variation of room acoustic transfer functions and its effects on a multi-microphone dereverberation approach," in Proc. Wrkshp. Microphone Arrays: Theory, Design and Applcn., Oct 1994.

[144] Y. Yai, S. Miyabe, H. Saruwatari, K. Shikano, and Y. Tatekura, "Rapid compensation of temperature fluctuation effect for multichannel sound field 
reproduction system," IEICE Trans. Fundamentals, vol. E91-A, no. 6, pp. 1329-1336, 2008.

[145] P. Coleman, P. Jackson, M. Olik, and J. A. Pederson, "Optimizing the planarity of sound zones," in Proc. 52nd Audio Eng. Soc. Int. Conf., (Guildford), pp. 1-10, Sep 2013.

[146] S.-R. Lee and K.-M. Sung, "Generalized encoding and decoding functions for a cylindrical ambisonic sound system," IEEE Signal Processing Letters, vol. 10, Jan 2003.

[147] A. N. Tikhonov, "Solution of incorrectly formulated problems and the regulation method," Sov. Math., vol. 4, pp. 1035-1038, 1964.

[148] G. A. Deschamps and H. S. Cabayas, "Antenna synthesis and solution of inverse problems by regularization methods," IEEE Trans. Antennas $\&$ Propagation, vol. AP-20, no. 3, pp. 268-274, 1972.

[149] C. E. Rasmussen, "Minimize," 2006.

[150] R. T. Rockafellar, Convex Analysis. Princeton University Press, 1970.

[151] Y. Saad, Iterative methods for sparse linear systems. SIAM, 2 ed., 12003.

[152] R. T. Rockafellar, Conjugate Duality and Optimization. Soc. Industrial \& Applied Mathematics, 1974.

[153] B. Rafaely, "Spatial-temporal correlation of a diffuse sound field," J. Acoust. Soc. Am., vol. 107, Feb 2000.

[154] H. M. Jones, R. A. Kennedy, and T. D. Abhayapala, "On dimensionality of multipath fields: Spatial extent and richness," in Proc. IEEE Conf. Acoust., Speech \& Signal Processing, vol. 3, pp. 2837-2840, 2002. 